\title{
Additive manufacturing of biomaterials
}

\author{
Susmita Bose*, Dongxu Ke, Himanshu Sahasrabudhe, Amit Bandyopadhyay* \\ W. M. Keck Biomedical Materials Research Lab, School of Mechanical and Materials \\ Engineering, Washington State University, Pullman, WA 99164, United States
}

\begin{abstract}
Biomaterials are used to engineer functional restoration of different tissues to improve human health and the quality of life. Biomaterials can be natural or synthetic. Additive manufacturing (AM) is a novel materials processing approach to create parts or prototypes layer-by-layer directly from a computer aided design (CAD) file. The combination of additive manufacturing and biomaterials is very promising, especially towards patient specific clinical applications. Challenges of AM technology along with related materials issues need to be realized to make this approach feasible for broader clinical needs. This approach is already making a significant gain towards numerous commercial biomedical devices. In this review, key additive manufacturing methods are first introduced followed by AM of different materials, and finally applications of AM in various treatment options. Realization of critical challenges and technical issues for different AM methods and biomaterial selections based on clinical needs are vital. Multidisciplinary research will be necessary to face those challenges and fully realize the potential of AM in the coming days.
\end{abstract}

\section{Keywords}

Additive manufacturing/3D printing; Bioceramics; Metallic biomaterials; Biopolymers; Drug delivery; Tissue engineering

\section{Introduction}

Additive manufacturing ( $\mathrm{AM}$ ) means processing or prototyping approaches that are capable of fabricating metallic, polymeric, ceramic or composite structures in a layer-by-layer manner from a computer generated design file. AM is also referred to as 3D Printing, Solid Freeform Fabrication (SFF), Layered Manufacturing (LM) or Rapid Prototyping (RP). In any AM process, parts are first designed using a computer aided design (CAD) software. Surface features of the three-dimensional CAD files are then exported to a file typically with a .STL extension. The .STL file is the main input file for an AM fabricator where the part is built. The surface file is sliced in a virtual environment into many two-dimensional (2D) layers. An AM machine then uses those 2D layers of the design file and creates the necessary tool-path along the $\mathrm{X}$ and $\mathrm{Y}$ directions for direct manufacturing. Finally, each layer is processed sequentially one on top of the other to form a three-dimensional part. Since each part is fabricated by adding layers on top of a previous layer, this type of 
manufacturing approach is called "additive manufacturing (AM)". AM fabricators utilize many conventional manufacturing techniques to build each layer. For example, Fused Deposition Modeling (FDM) is one of the most popular AM methods for polymeric materials. FDM works basically by softening a thermoplastic polymeric material and then extruding it through a nozzle to create a layer. Extrusion is a common manufacturing technique for polymers. However, extrusion of multiple layers based on a computer file to create a 3D object is the novelty for FDM. Thus, it can be seen that additive manufacturing borrows conventional manufacturing concepts and utilizes them in a non-traditional way to directly build 3D parts without using any part-specific tooling.

However, there are many differences between additive and conventional manufacturing. Conventional manufacturing processes are evolved to manufacture a large variety of parts as fast as possible, maybe even in high volume. Starting from raw materials to a finished part, processes are optimized for the highest yield. A simple example of such manufacturing principles is the steel industry, where high volume, low-cost fabrication processes like casting, forging or rolling are commonly practiced. After fabrication, parts are machined per customer requirement. In this machining stage, the material is removed and sometimes can be expensive as well as time-consuming. Finally, different components are assembled into a single system. The entire process from the design stage to the actual part realization is long but cheap for large volume production. AM, on the other hand, is a material-specific and design-specific system. Realization of high yield and low cost are not always mandatory. AM methods are unique in the situations where the production volume is not high, the cost of production is not the biggest concern but the part design realization and its application are the primary governing factors. In terms of the difference in the manufacturing process, AM methods are not designed, with the exception of a few, for large volume production. Specifically, most AM setup can fabricate only a few parts of smaller volumes or lesser number of parts of larger volumes. A machine at times can be capable of fabricating more than one component but the material system will be the same. Therefore, the cost of manufacturing is independent of the volume of production to some extent. As an example, consider that the build chamber of a machine is $1000 \mathrm{~cm}^{3}(10 \mathrm{~cm} \times 10 \mathrm{~cm} \times 10 \mathrm{~cm})$ and the part to be fabricated is $800 \mathrm{~cm}^{3}$, the machine is capable of processing only a single part at a time and will take a long time along with more feedstock consumption. And if a hundred such parts are required to be manufactured, the cost of manufacturing will be the same for each of those hundred parts and the time required will be the time for hundred parts will be the time required for one times hundred- unless a hundred machines run simultaneously or there is machine that has a very large build volume to accommodate more than one part; both scenarios pointing towards more initial machine cost. Now consider that the volume of the part is $100 \mathrm{~cm}^{3}$, then theoretically, each machine can fabricate ten parts with more efficient consumption of the feedstock material and therefore the cost of production can drop - all relative to the former example. The relationship between the cost of production and the volume of production is therefore dependent on the capabilities of each machine, the size of each part and also the material cost. On the contrary, for conventional manufacturing processes such as casting, the cost of manufacturing will always tend to go down with increasing volume of production. Typically, the size of the casting only has a significant effect on the initial die making operation and not so much on the following operations in 
production such as post-machining. The current efforts in additive manufacturing are focused towards bringing such production costs down and making the processes more efficient and competitive even for a large volume of parts compared to different conventional approaches.

Biomaterials are natural or synthetic materials that are useful towards the repair of damaged body parts via interacting with living systems. These materials are used to either replace a component of the human body or support physiological functions. As such, biomaterials interact with the human cells or tissues or organs and sometimes even carrying out their functions. Over the last five decades, all classes of materials including metallic, ceramic, polymeric and composite have drawn engineers and scientists for biomedical applications. Initially, there was very little understanding of "biocompatibility" of materials. However, the current situation is different. With the advancement of our understanding of biological properties that are desired in a material for biomedical applications, unique material properties are constantly pursued to cure different diseases to improve the quality of human life. Naturally, manufacturing of biomaterials is becoming an important topic to improve reliability and reduce the risk of rejection by the human body. Advanced manufacturing techniques are constantly being explored to process biomaterials to reduce cost, minimize inventory and maximize performance in vivo. Apart from single component manufacturing, advanced manufacturing sciences are also being explored to produce multi-part structures that are difficult to manufacture using conventional routes. Such structurally or compositionally gradient parts can act as multifunctional biomedical devices and improve in vivo performance.

An example of this combined necessity of properties can be seen in a hip stem of a total hip replacement. The metallic hip stem must possess the right blend of mechanical strength, structural stability from fatigue loading, and biocompatibility. Furthermore, for better integration with the surrounding bone tissues, a porous metallic outer layer or a bioceramic coating are desired. Because of such complex combinations of materials and structures, multiple processing steps are necessary to manufacture these hip stems. Typically, the metallic components are first made using techniques such as casting or forging. Before the actual parts are processed, a pattern has to be created. This pattern is then used to create the mold in the shape of the required part. After processing is done, the part has to be machined to remove excess materials. Following this machining treatment, the part has to be machined again to attain the desired surface finish. Many times, to obtain the desired properties, postprocessing heat treatment may be needed. If specialized surface properties are required, a secondary process can be used. For example, if a bioceramic such as calcium phosphate coating is required over the conventionally processed part, a process such as plasma spray is used to deposit the ceramic coating. However, if the parts are produced via additive manufacturing, the highest costs involved are the costs of the AM machine and raw materials. Comparing the process setup, there are no costs involved in specific pattern making or tooling. After AM based processing, machining may be needed to get the desired surface finish. Key advantage towards AM of biomedical devices lies in patient-specific device manufacturing. In some AM approaches, secondary processing such as depositing a bioceramic coating on a metallic hip stem can also be integrated. 
However, there are some disadvantages of AM methods apart from the initial setup costs. Most additive manufacturing methods use metallic or ceramic powder materials. The costs of such specialized powders can be high. Polymeric materials are comparatively cheaper and most are used in the form of wire or filament in AM methods although many are used in powder forms or via a suspension or a solution. AM machines also need to be optimized for the specific material. This process is essential to ensure that the properties that result from processing are reproducible. From a production point of view, there is a need for having different machines or individual machines for each material to avoid contamination. This can further increase the investment needed for industrial scale production.

\section{Classification of additive manufacturing techniques}

There are two main categories of additive manufacturing techniques for biomaterials - (1) acellular and (2) cellular. The acellular category involves printing materials without any live cells. The cellular category involves the printing of live cells along with other materials. The American Society for Testing of Materials (ASTM) has provided a guideline for classification of different additive manufacturing techniques [1]. Fig. 1 schematically shows different AM techniques for biomaterials. Also, the following section briefly discusses some of the important AM techniques that are used to process biomaterials.

\subsection{Binder jetting}

In binder jetting technique, the liquid binding agent is spread to a selected area on the powder bed based on the part geometry to form a designed structure layer by layer [1]. A typical binder jetting system has three axes where $\mathrm{x}$ and $\mathrm{y}$ are responsible for horizontal position, and $\mathrm{z}$ - axis refers the depth for $\mathrm{x}$ and $\mathrm{y}$. Commercial binder jetting systems are available from ExOne and Voxeljet. Binder jetting requires an understanding of many process variables for a successful build, which is briefly described in the following section.

2.1.1. Powder selection-A broad range of materials such as sand, ceramic and metal, which can be formed into appropriate powders for certain binder interaction, can be used in binder jetting. In addition, binder jetting is able to be conducted not only on dry powders, but on wet powders as well. The requirements for dry and wet powder binder jetting are very different. However, there are two mutual important factors which can affect the binder jetting printing process, as shown in Table 1.

- Powder size: Powder size has significant effects on powder flowability. Generally, large particles are preferred by dry binder jetting because of its excellent flowability and low surface area. Fine powder is usually spread as slurry because it is easy to absorb moisture due to the high surface area, which leads agglomeration and affects powder flowability [8]. However, there was a contradictory study which reported parts prepared by binder jetting using fine powder $(<20 \mu \mathrm{m})$ also had high mechanical strength [9]. Besides effects on powder flowability, powder size also has huge influences on the property of final parts. Binder jetting by fine powder is able to improve the density of final parts after the post processing, such as sintering. In addition, fine powder binder jetting can offer better surface finishing and low surface roughness. 
- Powder shape: Powder shape has less influence on binder jetting than particle size. However, for dry binder jetting, spherical powders are preferred because of its better flowability and lower friction than faceted or anisotropic powders $[6,7]$.

Coarse and fine powders can also be applied together in binder jetting in order to gain advantages from both large and small particles. The large particles will guarantee the flowability under dry binder jetting when the small particles fill voids between large particles to increase the packing density and inherit advantages via using fine powders [10]. However, the ratio of the fine powder to coarse powder needs to be optimized in case that the addition of fine powder decreases the flowability of the mixture, which will be deleterious to the powder packing. Zhou et al. reported a study for effects of particle size on binder jetted calcium sulfate/calcium phosphate scaffolds. Interestingly, the addition of fine calcium phosphate powder did not increase the bed packing density. The flowability was the predominant factor, which leads rougher surface and low packing density due to the addition of the fine binder as shown in Fig. 2 [11].

2.1.2. Binder selection-There are two types of binders - organic and inorganic. An organic binder binds the powder through the curing process, while the inorganic binder binds via colloid gel formation [12]. Binders can also be classified as acid-base binder, metal salts binder, and solvent binder. Acid-base binder controls the powder bonding through acidbase reaction. Metal salts binder generally forms the bonding with powder by salt recrystallization, reduction of salt crystallization or salt displacement reaction. The solvent binder is specialized for polymeric powders, which can dissolve the deposited area and create designed structure after solvent evaporation. In addition, based on different binding mechanisms, there are in-bed binders, phase-changing binders, and sintering inhibition binders. The in-bed binder is different from normal liquid binder due to the source of binder. In-bed binder mostly mixes with the powder in the bed and then binds with powder through the jetting liquid from the nozzle. Typical examples are plasters and cement, where the binding is formed via hydration-activated bonds when liquid jets on the powder bed $[3,13,14]$. Phase-changing binder works by solidification of binder to hold the powder together. Finally, sintering inhibition binder can control the sintering area via selectively jetting heat-isolating material. Table 2 summarizes different binders to understand their advantages and disadvantages.

2.1.3. Printing-The main purpose of the printing process is to inkject binder from the jetting head to the build stage for selective binding. As for the machine itself, it can be separated into four parts - feed stage, leveling roller, build stage and binder jetting head. The function of feed stage is to have dry powder or slurry for powder feeding. For dry powder binder jetting, the powder must be dry and compact in order to have a good layer spreading, which indicates deposited layers with smooth surface and uniform thickness [32]. For wet powder binder jetting, the solid is mixed with a liquid carrier to form a homogeneous slurry. Sometimes, dispersants are added to decrease the viscosity and to increase solids loading of the slurry. After formation of an excellent powder packing or slurry forming on the powder bed, leveling rollers are needed for feeding the powder from the feed stage to the build stage. There are various types of leveling rollers for different applications. For dry powders, the 
most common one is traversing counter-rotating roller, which rotates the opposite direction to the traversing direction and is able to feed new layers from the powder bed without disturbing the previous layers [24]. This kind of roller can also be vibrated and charged to assist in powder flow [32,33]. Other rollers for dry powder spreading are charged plates, traversing doctor blades and rotating sieve drums [7,34]. Liquid slurry is usually spread by slip casting where the rheology of the slurry is very important. After each layer of slurry feeding, the liquid carrier needs to dry out first in order to expose all solid material. The packing density for slurry feeding is usually higher than dry powder feeding, but slurry feeding has a longer build time and a greater risk of cracking during the printing process $[35,8]$.

The next step is to print the designed structure. There are two types of print heads: drop-ondemand (DoD) and continuous-jet (CJ). DoD print heads print structure using drops of liquid, which are dispensed on demand. Two major DoD print heads are thermal ink jet and piezoelectric. The mechanism for thermal inkjet head is to vaporize binder in the print head for creating volume expansion followed by jetting of the ink [36]. For the piezoelectric head, it is able to squeeze the ink due to piezoelectric actuation [36]. CJ type print heads can produce continuous jetting of ink. Detailed information about different print heads along with their advantages and disadvantages are listed in Table 3.

After printing each layer, the build stage goes down for the next layer of printing. During printing, binder saturation level represents the ratio of ink to bed pour volume, which is dependent on droplet spacing and size, bed packing density and layer thickness [39].

Saturation level shouldn't be too high, as it might lead to excess powder binding causing uneven surface on the build bed; however a low saturation level can result in insufficient binder penetration into the powder [13,40]. Drying time is also an important factor, where enough drying time is needed to let binder fully bound with powder for preventing cracking during printing. Finally, the preferred layer thickness should be at least three times that of the powder particle size [8]. If the powder is a composite, the layer thickness should be higher than the largest particle size of the composite powder to achieve excellent powder spreading [3]. The printing process can be visualized from Fig. 3 [41].

2.1.4. Post-processing-After printing, in order to get the part, the excess powder should be removed from the part followed by heat treatment and in some cases, infiltration. The goal of the post-printing process is to enhance bonding between powder and binder in order to improve the green strength of the parts. For some polymer binder, curing is one possible solution to increase the bonding strength via further polymerization without influencing the loose powder $[42,43]$. Another method for enhancing the mechanical strength is by drying because it removes the extra liquid in the part, especially for cement and plaster-based materials [14]. Besides these two, other options include selective sintering with sintering inhibitors [31], reduction of a salt-based binder [27] and pre-ceramic polymer conversion [19]. After the part has enough mechanical strength, depowderization is normally conducted to remove the loose powder from the part. Options, such as vibration, vacuuming, and wet depowderization, are commonly utilized methods [38,42,44]. Additional processes such as microwave boiling [45], carbon dioxide bubbling [46] and ultrasonication [40] can be assisted with wet depowderization. After depowderization, parts are heat treated or 
sintered to improve density and mechanical property. For sintering methodologies, conventional and microwave sintering are two commonly practiced approaches. Conventional sintering is done in a regular furnace via surface heating however, microwave sintering is done in a microwave furnace through the absorption of microwave energy. It has been shown that microwave sintering can significantly increase the density and compressive strength of porous tricalcium phosphate (TCP) parts by binder jetting compared to conventional sintering and shown in Fig. 4 [47].

Some sintered parts are infiltrated with a second material to further improve mechanical properties. Infiltration can be divided into low-temperature and high-temperature processes. The materials for low-temperature infiltration are mostly polymers such as epoxy [14] and polycaprolactone (PCL) $[48,49]$. However, many alloys can be infiltrated at high temperature. It was reported that a $\mathrm{SiC}-\mathrm{Si}$ composite part was prepared by carbon powder printing followed by $\mathrm{Si}$ infiltration at $1450{ }^{\circ} \mathrm{C}$ with the assistance of vacuum [50]. Besides mechanical improvement, the infiltration also provides a new way to fabricate composite parts.

\subsection{Direct energy deposition techniques}

In direct energy based techniques, a direct source of energy is used to simultaneously deposit, melt and solidify the material that is either fed as a powder or as a filament or wire. The "direct energy" source is typically laser or electron beam. These processes depend on the continuous feeding of the starting material either in the form of powder or filament that are then melted by the energy source and deposited in a layer-wise pattern. A computer controlled XYZ system controls the part geometry and build pattern in these processes. The processes coming under this category are mainly applied to metals but in some instances have also been applied to polymeric, ceramic and composite materials. The most popular processes that fall under this technique are Laser Direct Deposition techniques that encompass: laser cladding, laser melt injection and laser engineered net shaping (LENS ${ }^{\mathrm{TM}}$ ). Processes like electron beam direct manufacturing (EBDM) also fall under direct energy deposition techniques. For better organization of different techniques under this category, various approaches are grouped either as techniques for surface modification or for 3D part fabrication as shown in Fig. 5. Surface modification can be achieved using additive manufacturing routes for improving the surface performance of the material whereas 3D part fabrication means near net shaped functional component manufacturing.

2.2.1. Laser melt injection and laser cladding - In all of these techniques, a focused high-power laser is used to melt and solidify materials onto a substrate. Some of these techniques are used for developing different types of coatings or surface modification, whereas some can be used for fabrication of a complete part. All of these techniques resemble each other in terms of material injection and motion control. The earliest form of surface modification using the term "laser cladding" or "laser melt injection" was simpler and slightly different than the current methods. These were processes in which powder was preplaced and compacted on the surface of the substrate. The powder was heated using a high powered laser surface scanning. This scan caused melting and bonding of the powder to the substrate along with localized sintering. With the advancement in computer numeric 
control (CNC) systems and powder/wire delivery systems, these methods soon evolved into the current day techniques of powder-based additive manufacturing.

Laser melt particle injection was one of the earliest precursors of the modern powder based direct energy additive manufacturing. Ayers et al. showed how the surface of the metallic substrate can be micro-melted and fine ceramic particles can be injected into this micro melt pool. Similar to the laser melt injection in which powder is injected into the micro-melt pool, laser cladding can also be done [51]. Both laser melt injection and laser cladding can be done using material feed in the form of powder, wire or filament. Fig. 6 shows typical laser melt injection using powder feed and laser cladding using a wire feed [52,53]. Using a computerized motion control, the material can be deposited along $\mathrm{X}, \mathrm{Y}$ and $\mathrm{Z}$ axes as well as on rotating parts, both inside and outside.

2.2.2. Direct laser deposition-Direct laser deposition techniques use a feed of material, either in a powder form or wire/filament form, to form a three-dimensional part. These techniques can also be used for selective surface modification of materials. Another way of collectively recognizing these methods is laser powder forming or laser based metal deposition (LBMD) [54]. Laser Engineered Net Shaping (LENS ${ }^{\mathrm{TM}}$ ) is a commercially available approach that belongs to the class of direct laser deposition techniques.

LENS $^{\mathrm{TM}}$ technology was originally developed by Sandia National Laboratory (Albuquerque, NM), and commercialized by Optomec Inc. (Albuquerque, NM). LENS ${ }^{\text {TM }}$ is equipped with a high power laser (up to $4 \mathrm{~kW}$, solid state or fiber optic laser). There are multiples powder feeders available, and a mixture of powders can be fed through Argon pressurized nozzles. The powder is delivered through multiple delivery nozzles into a converging point which is also the focal point of the laser. Upon delivery, the powder melts and forms a micro-melt pool. This melt pool adheres to the substrate/base plate that moves along the $\mathrm{X}$ and $\mathrm{Y}$ axes. As the base plate moves, the system creates a line of molten material that is instantly solidifying to create a cross-sectional layer. Once the layer is done, the nozzle and the laser assembly move up along the $\mathrm{Z}$ direction by one layer thickness and deposits another layer on top of the previous layer. Such a repeated layer-wise deposition of material creates a threedimensional (3D) component based on the original CAD file. The entire build assembly in a LENS $^{\mathrm{TM}}$ system is enclosed in a glove box under a controlled environment. This helps to prevent any oxidation of build material during deposition. A schematic of the LENS ${ }^{\text {TM }}$ technology is shown in Fig. 7 [55].

2.2.3. Electron beam direct manufacturing-Electron beam direct manufacturing or EBDM uses an electron beam to melt materials and deposit them in a 3D pattern similar to that of direct laser deposition technique. The materials in the EBDM process are fed in the form of a wire or a filament. Since electron beam is the direct energy source in the EBDM process, the process is carried out under high vacuum to prevent the ionization of the atmospheric gases. This eliminates the need for using an inert gas like argon typically used in laser-based processes since the use of vacuum eliminates any other contaminants such as oxygen or nitrogen that may enter the material from the atmosphere. Commercially, EBDM process is available from Sciaky Inc. (Chicago, Il, USA) under the brand name Electron Beam Additive Manufacturing (EBAM $\left.{ }^{\mathrm{TM}}\right)$. 
Different processes discussed under the direct energy deposition technique for additive manufacturing have been demonstrated successfully for a variety of metallic materials such as steels, super alloys, Co-based alloys, Ti based alloys and bulk metallic glasses. Many sensitive materials such as pure $\mathrm{Ti}, \mathrm{Si}, \mathrm{Ta}, \mathrm{W}$ and $\mathrm{Zr}$; ceramic materials such as alumina, zirconia, and different carbides and composites of metal and ceramics have also been demonstrated [56-60]. Apart from the different material systems that these techniques are capable of processing, these techniques have also been utilized for fabrication of structurally graded materials with differing porosity in different parts of the components [61], compositionally graded materials and manufacturing of multi-material systems [62-65].

\subsection{Material extrusion and jetting}

In this approach, extrusion of material is utilized to form a 3D part. This process is commonly known as fused deposition modeling (FDM) [66]. FDM technology is mainly applied for direct 3D printing of plastics or polymeric materials. This is due to the relatively lower softening temperature of polymeric materials as compared to metallic or ceramic materials. The FDM is a hot extrusion method in which a material is heated up until it flows. In thermoplastic polymers, the material softens during heating and hence can be extruded or squeezed through a nozzle. The extrusion rate and resolution are controlled for different builds using a pre-determined nozzle. When the material is extruded in a layer-wise fashion comprising consecutive raster scans in each layer, a 3D part, based on a CAD design, can be formed. Fig. 8 shows a schematic of the FDM process [67]. A modified version of the FDM process, called fused deposition of ceramic (FDC), has also been used to process green ceramic structures [68]. Controlled by the limitation of the applicable material, FDM technology can only fabricate polymer parts. However, ceramic constructs can also be prepared using indirect FDM as reported by Bose et al. [69]. A polymer mold with honeycomb structure was first made by FDM. Then the ceramic slurry was infiltrated to the mold followed by the sintering to remove the mold. Ceramic parts with controlled pore size and pore porosity were successfully prepared using this indirect FDM method. The whole processing schematic and part microstructure were shown in Fig. 9 [69].

A deviation from additive manufacturing using material extrusion is the process that uses material jetting. In material jetting process, droplets of polymeric material are deposited on a powder bed to form a 2D pattern. These deposited droplets are then immediately cured using ultraviolet radiation. Fine layers can be formed in this manner and the process is repeated for a number of layers to form a complete 3D part. This technology was introduced by Stratasys Inc. under the name PolyJet in 2007. There are also secondary nozzles for jetting the droplets of secondary materials as a support material, much like the support structures created in the FDM processes. The PolyJet technology (Fig. 10) has also demonstrated capability to create combinations of different materials, something that was not previously achievable using the FDM technology [70].

\subsection{Powder bed fusion}

Powder bed fusion utilizes thermal energy to fuse certain areas on the powder bed. The advantages include its high dimensional accuracy for making small complex parts, fabrication without supports, and a broad range of printable powders. This process is similar 
to dry powder binder jetting except the use of thermal energy to bind the powder instead of a jetting binder. Powder bed fusion (PBF) is one of the first generation of commercialized additive manufacturing processes. Within PBF, selective laser sintering (SLS) was the first PBF process developed and commercialized [71]. Selective laser melting (SLM) is another PBF process [72]. Newer PBF technologies, such as direct metal laser sintering from EOS (Krailling, Germany), electron beam melting from Arcam AB (Mölndal, Sweden) and laser curing from CONCEPT LASER (Lichtenfels, Germany), were also developed for different application fields [73].

The printing process for powder bed fusion is following similar steps as the binder jetting. A schematic of the process is shown in Fig. 11 [74]. Briefly, the energy source fuses the powder after each layer feeding, which results in the construction of the 3D structure via layer-by-layer deposition. However, during the printing process, there are some significant differences compared to binder jetting. Before printing, a preheating process is necessary till it reaches the temperature slightly below the glass transition or melting point of the powder, which can decrease the power requirement of the energy source and facilitate powder fusion [71]. Initial heating is also beneficial to decrease the temperature gradient and minimize the thermal distortion in the final part.

Another important difference is the printing environment. Unlike the open environment in binder jetting, an oxygen free environment is needed for powder bed fusion to prevent oxidation of the feedstock powder. Some protective gas environments, such as nitrogen for non-reactive powder, argon for reactive powder, and vacuum for electron beam source, are common for powder bed fusion $[74,75]$. According to different thermal energy sources, powder bed fusion can be further divided into laser and electron beam based powder bed fusion.

Laser Energy: For the laser powder bed fusion, it can be further separated into selective laser sintering (SLS) and selective laser melting (SLM). The differences are their powder fusing mechanisms and material options. SLM fully melts the material and forms a homogeneous part. It is a single material process since the melting points of different materials are not be the same, but alloyed powders can be used. However, for SLS, the laser heats the powder to a point that it can molecularly fuse together at the surface. Table 4 lists different laser-based PBF technologies.

Electron Beam Energy: Electron beam melting (EBM) uses electron beam as the thermal energy source to selectively fuse powders together. The process is very similar to SLM except electron beam uses vacuum environment to build [76]. The EBM is a faster process compared to SLM. The main purpose of using vacuum environment is to improve the quality of the electron beam and prevent the contamination of powder or the built material due to the atmospheric gases [77]. Table 5 shows a direct comparison between the SLM and the EBM processes [74].

Post-processing: The most important post-processing for PBF is slow cooling. A slow cooling to the room temperature is crucial to prevent warping and cracking. Decreasing the cooling rate is an effective way to minimize thermal distortion, but it takes longer time. 
Other post-processing steps such as polishing, machining, hot isostatic pressing, shot peening and heat treatment are also used depending on applications of AM fabricated parts [74].

\subsection{Vat polymerization}

Vat polymerization, the first commercially available additive manufacturing technique, produces parts via selectively curing liquid photo-reactive polymers. It is commonly known as stereolithography (SLA). This method is featured with high building accuracy, fast building rate and excellent part quality. The resolution of vat polymerization can reach as high as $200 \mathrm{~nm}$ with excellent surface finish [78]. In addition, this process offers options to manufacture parts in large size with different materials such as polymers, metals, and ceramics.

Polymerization Reaction and Photo-reactive Materials: There are two major types of polymerization for vat polymerization, free-radical and cationic polymerization. Freeradical polymerization happens when photo-reactive polymers first form long chains and then get close to each other followed by crosslinking reaction. The most common photoreactive material for free-radical polymerization is acrylates. This method offers high reaction speed, but challenges include high shrinkage and mechanical failure including curling and warping [79]. Another reaction usually starts with a polymer with ring structure such as epoxies. When cationic polymerization happens, those rings open leading to active positions for other chemical bond formation [80]. Since the number and structure of the new bonds are very similar to the original chemical bond, it results in little curling, warping, and shrinkage. Sometimes we can also combine these two different polymerization methods to achieve faster build with excellent part quality. Besides photo-reactive materials, photoinitiators are also crucial to decrease the requirement of thermal energy for polymerization. Its function is similar to a catalyst in chemical reactions. Fig. 12 shows equations for free-radical and cationic polymerizations. " $R$ " usually is a functional branch including vinyl groups [80]. The crosslinking mostly happens at $\mathrm{R}$ sites under vat polymerization. Table 6 summarizes photo-reactive polymers and photoinitiators for vat polymerization.

Processing Steps: The general process starts from the formulation of the solution in the vat. Sometimes dispersants are mixed with other necessary components to achieve good part quality. Then according to the CAD file, the radiation pattern is reflected on the solution surface to initiate polymerization of photo-reactive materials. After curing of one layer, the platform moves down for the polymerization of another layer on top till the entire process is done. During the process, two factors, cure depth and cure width, are important to control to achieve high-quality parts $[81,92]$. There are three configurations for the radiation sources, which is worth mentioning for understanding differences during the polymerization process. Fig. 13 shows the schematic of those three set-ups [71]. The vector scan is like the early vat polymerization process. One laser beam works as the radiation source through the focus and projection using optics and a scanning galvanometer for polymerization in the vat. The advantage of this method is its high resolution and excellent surface finish. The second method, mask projection, is different than a vector scan method. Instead of curing from one 
point to the next one, the radiation source can generate a large pattern using a digital micromirror device (DMD) and cure one layer at a time. Hence the most significant advantage of this method is the high building speeding. The last method is called two-photon vat polymerization, which indicates that two laser beams get involved during the process. Curing only starts at the intersections of two laser beams because of its high energy requirement. Besides this difference, the two-photon method doesn't need a build platform as polymerization can be accomplished inside a solution. This method further improves the resolution of the process since only the center of the intersection for the two laser beams has enough radiation energy to active polymerization [93].

Post-processing: Post-processing steps include three primary sections - cleaning, postcuring and finishing. Cleaning includes removing the extra solution and supporting material around the part. After this process, parts are cured further under radiation source for achieving a fully polymerized part with better mechanical properties. Finally, the secondary surface finishing operation can be conducted using vapor honing, and bead or sand blasting based on application needs.

\section{Additive Manufacturing (AM) of different biomaterials}

\subsection{Additive manufacturing of ceramics}

Ceramic biomaterials, namely bioceramics, are relatively new developments for AM because of their inherent processing difficulties. Firstly, melting points of ceramic materials are generally too high to melt under normal heating methods. Even though we can melt some ceramics, they usually have complex phase diagrams, which indicates that the melting of these ceramics might cause new phase formation and change their resorption ability. For example, $\beta$-tricalcium phosphate ( $\beta$-TCP) can transform to $\alpha$-TCP under the temperature above $1100^{\circ} \mathrm{C}$. Compared to $\beta$-TCP, $\alpha$-TCP is more soluble and biodegradable, which has a significant influence on its final applications. Secondly, the high-temperature processing of ceramics can cause porosities and cracks. There are three types of porosities, microporosity, mesoporosity, and macroporosity. They can all decrease the mechanical properties of final parts, but they are also beneficial for cellular growth and implant fixation. Hence, a balance between mechanical properties and biological properties should be found for different applications. Some other pore parameters, such as pore geometry, pore thickness, and pore permeability have significant effects on the through-flow capability, which has influences on the resorption rate of final ceramic parts. Cracks are usually formed during the rapid heating and cooling due to the high residual thermal stress/strain. It can significantly decrease the mechanical properties of final ceramic parts. However, ceramic materials play an important role as biomaterials. The best examples for the application of bioceramics are bone and teeth. Almost $70 \%$ of bone is made of ceramic, whose chemical composition is very close to calcium phosphate $(\mathrm{CaP})$. Similarly, teeth also have a large amount of $\mathrm{CaP}$ content. $\mathrm{AM}$ has advantages of small-scale parts manufacturing with complex design and low cost, which is beneficial to the patient specific needs for bone and teeth replacement. Even though traditional manufacturing can also make accurate molds for producing complex parts, sometimes the cost and time to make mold are substantial. This section will describe 
different bioceramics, such as calcium phosphates, bioglass, and ceramic composites, and their processing using various AM technologies.

3.1.1. Calcium phosphate (CaP) ceramics $-\mathrm{CaP}$ is a group of ceramic materials with calcium ions $\left(\mathrm{Ca}^{2+}\right)$, different phosphate ions, including metaphosphates $\left(\mathrm{PO}^{3-}\right)$, orthophosphates $\left(\mathrm{PO}_{4}^{3-}\right)$ and pyrophosphates $\left(\mathrm{P}_{2} \mathrm{O}_{7}^{4-}\right)$, and sometimes hydroxide or carbonate ions. Because of its compositional similarity with bone and teeth, CaPs are popular bioceramic materials. However, significant differences exist in physical, mechanical and biological properties of various $\mathrm{CaPs}$. $\mathrm{Ca}$ to $\mathrm{P}$ ratio is one important factor that influences the dissolution property of $\mathrm{CaPs}$ where the dissolution rate of $\mathrm{CaPs}$ increases with a decrease of $\mathrm{Ca}$ to $\mathrm{P}$ ratio $[94,95]$. Low $\mathrm{Ca}$ to $\mathrm{P}$ ratio leads an acidic environment and increases $\mathrm{CaPs}$ dissolution rate. The bioactivity of $\mathrm{CaPs}$ is also controlled by $\mathrm{Ca}$ to $\mathrm{P}$ ratio. $\mathrm{A}$ $\mathrm{Ca}$ to $\mathrm{P}$ ratio close to 1.67 represents hydroxyapatite $\left(\mathrm{Ca}_{10}\left(\mathrm{PO}_{4}\right)_{6}(\mathrm{OH})_{2}\right)$, which has shown excellent biocompatibility due to similarity with the composition of natural bone and teeth. However, $\mathrm{Ca}\left(\mathrm{H}_{2} \mathrm{PO}_{4}\right)_{2} \cdot \mathrm{H}_{2} \mathrm{O}$ (Monocalcium phosphate monohydrate, MCPM), whose $\mathrm{Ca}$ to $\mathrm{P}$ ratio is 0.5 , is not biocompatible at all because of its high acid nature. In addition, it was reported that the $\mathrm{Ca}$ to $\mathrm{P}$ ratio between one and two is beneficial for osteoblast cells viability and the alkaline phosphatase production [96]. Table 7 lists the dissolution behavior and $\mathrm{Ca}$ to $\mathrm{P}$ ratio of different $\mathrm{CaPs}$ materials $[95,97]$.

Hydroxyapatite (HA): $\mathrm{HA}$ is found in the bone and usually written as $\mathrm{Ca}_{10}\left(\mathrm{PO}_{4}\right)_{6}(\mathrm{OH})_{2}$ instead of $\mathrm{Ca}_{5}\left(\mathrm{PO}_{4}\right)_{3}(\mathrm{OH})$ because it contains two entities in one single crystal. The $\mathrm{Ca}$ to $\mathrm{P}$ ratio of $\mathrm{HA}$ is 1.67 , which has better biocompatibility than other CaPs. Many AM approaches have been used to make HA based parts for biomedical applications.

Vat polymerization is an effective method for preparing complex HA parts by mixing HA powder with photoactive polymer and additives, curing the suspension using laser pattern and then heat treating the parts to remove polymeric binders followed by densification. Griffith et al. reported effects of HA loading percentage on the viscosity of the HA suspension and curing parameters for vat polymerization of HA [98]. The maximum HA loading used in this study was $45 \mathrm{vol} \%$. It was reported that ceramic loading percentage was inversely proportional to cure depth of penetration. Chu et al. reported a method using vat polymerization for making resin molds in order to fabricate HA implants with designed internal structures [99]. Pore channels of HA parts were between $368 \mathrm{~mm}$ and $968 \mathrm{~mm}$ in diameter. Porosity was in the range of $26 \%$ to $52 \%$. This method successfully prepared crack-free internal structure with controlled channel size from microstructural images of implants cross sections. Woesz et al. presented a similar study of making interconnected porous HA structures using vat polymerization processed molds for bone replacement applications [100]. HA scaffolds with $450 \mu \mathrm{m}$ of pore size were fabricated using this method. Final scaffolds were also tested with preosteoblastic cell line MC3T3-E1 for 2 weeks. Excellent osteogenesis by cell staining was reported, as shown in Fig. 14 [100]. For those two studies, vat polymerization was not utilized directly on HA, but on the photoactive polymer to make a mold. Then the final HA part was prepared through ceramic gel casting using the mold followed by sintering to get rid of the mold. 
Shuai et al. reported a laser powder bed fusion method to manufacture bone tissue engineering scaffolds using nano-HA powder [101]. Nano-HA based bone scaffolds with a pore size of around $3 \mathrm{~mm}$ were prepared using SLS. The $50 \mathrm{~kW}$ laser power in SLS didn't decompose the HA and maintained the nanoscale structure with no cracks after the process. It proved the feasibility of using SLS to process HA constructs. Dense and porous HA scaffolds were prepared by Seitz et al. via binder jetting method followed by sintering at $1250{ }^{\circ} \mathrm{C}$ [102]. The compressive strength of dense parts was $22 \mathrm{MPa}$. Porous scaffolds were prepared featured with inner channels having a dimension down to $450 \mu \mathrm{m}$ and wall structures with a thickness of $330 \mu \mathrm{m}$, but the mechanical property of porous HA was not reported. Leukers et al. also reported porous HA scaffolds prepared by binder jetting method and their bioactivity characterized via static and dynamic culturing using MC3T3-E1 cells [103]. Under both culturing methods, HA scaffolds were biocompatible. However, samples cultured under dynamic culturing conditions showed deeper cell growth inside the scaffolds.

Material extrusion based AM has also been used for making HA scaffolds. In this method, the rheological property of HA suspension is very important for successful printing. HA loading should be as high as possible to reduce cracking and warping during sintering. However, high HA loading increases the viscosity, which makes the extrusion process difficult. Miranda et al. reported a robocasting method to prepare porous HA scaffolds for the mechanical property characterization by compression testing [104]. The HA slurry contained $35 \mathrm{vol} \% \mathrm{HA}, 6.5 \mathrm{wt} \%$ of Darvan C dispersant, an appropriate amount of hydroxypropyl methylcellulose, DI water, $0.4 \mathrm{wt} \%$ of polyethyleneimine (PEI) and $\mathrm{HNO}_{3}$ or $\mathrm{NH}_{4} \mathrm{OH}$ for adjusting the $\mathrm{pH}$. After making the part, scaffolds were sintered at $1300{ }^{\circ} \mathrm{C}$ for 2 h. The final scaffolds were featured with $39 \%$ porosity including around $28 \%$ macroporosity. The compressive strength under two different compression directions was $47 \pm 14$ $\mathrm{MPa}$ and $50 \pm 5 \mathrm{MPa}$. Dellinger et al. also reported a robocasting method using HA suspension for delivering bone morphogenetic protein 2 (BMP-2) [105]. The HA slurry composition was 48 vol\% HA, $1.25 \mathrm{wt} \%$ Darvan 821 A, 1.0 v/o 1-octanol, $2.5 \mathrm{mg} / \mathrm{mL}$ methocel F4M and DI water. Controlled microporosity was produced by mixing polymethylmethacrylate (PMMA) microspheres with HA slurry. Scaffolds were sintered at $1300{ }^{\circ} \mathrm{C}$ for $2 \mathrm{~h}$ to remove other contents. Then $10 \mu \mathrm{g}$ of BMP-2 was added to the scaffolds for in vivo characterization in goat metacarpal bone for 4 and 8 weeks. Results showed promising potential for making HA scaffolds with randomly interconnected porosity. In addition, the presence of BMP-2 and micro-porosity improved osteogenesis of the scaffolds as shown in Fig. 15 [105].

Tricalcium phosphate (TCP): $\mathrm{TCP}$ is another popular $\mathrm{CaP}$ material. It has three main phases, $a, a^{\prime}$ and $\beta$. $\beta$-TCP is the primary TCP phase at low temperature, while $a$ and $\alpha^{\prime}$ phases are formed at high temperature. TCP has a $\mathrm{Ca}$ to $\mathrm{P}$ ratio of 1.5 , which is lower than HA. Lower Ca to $\mathrm{P}$ ratio of TCP increases its biodegradation rate compared to HA. Wiltfang et al. reported a study using $a$ and $\beta$-TCP as biodegradable filling material in tibiae critical size defects of mini pigs [106]. After 28 weeks, between 70 and $80 \%$ resorption was reported for a-TCP and $\beta$-TCP, respectively, with newly formed trabeculae. Such results make TCP an exciting material towards the development of biodegradable ceramic implants. 
AM of TCP has been explored for fabricating complex patient-specific parts for bone tissue engineering applications.

Vorndran et al. reported a dry powder binder jetting method using $5 \mathrm{wt} \%$

hydroxypropylmethylcellulose modified $\beta$-TCP as the matrix material and water as the binder for making porous $\beta$-TCP scaffolds [107]. The final parts had low printing resolution low specific surface area and a maximum compressive strength of $1.2 \pm 0.2 \mathrm{MPa}$. Tarafder et al. reported effects of the microwave sintering and pore size on mechanical and biological properties of porous $\beta$-TCP scaffolds prepared by binder jetting [47]. Scaffolds with a 500 $\mu \mathrm{m}$ of designed pore size and $27 \%$ of designed porosity microwave sintered at $1250^{\circ} \mathrm{C}$ had $42.95 \pm 1.60 \%$ of bulk density and a maximum compressive strength of $10.95 \pm 1.28 \mathrm{MPa}$. In addition, porous $\beta$-TCP scaffolds with this design showed excellent biocompatibility in vitro using osteoblast cells and in vivo using a rat femur defect model, as shown in Fig. 16 [47].

Felzmann et al. reported a vat polymerization method for making porous $\beta$-TCP implants using acrylate-based monomers, dispersant, photoinitiator and $\beta$-TCP powder, but the $\beta$ TCP loading percentage was only $45 \mathrm{wt} \%$ [108]. The density and biaxial strength of final parts were $88 \%$ and $30 \mathrm{MPa}$, respectively, with a pore size around $300 \mu \mathrm{m}$. A recent report showed a novel method for making blood vessel implants with preset inside channels using vat polymerization [109]. The parts, which contained $70 \mathrm{wt} \% \beta$-TCP loading, were made without any deformation and crack. In addition, the sintered parts had $45 \%$ porosity and a compressive strength of $23.54 \mathrm{MPa}$, which is in the range of the compressive strength of cancellous bones. Yuan et al. reported a robocasting method to create porous $\beta$-TCP scaffolds for the anti-tuberculosis drugs release [110]. $\beta$-TCP, paraffin, and other additives were used as the ink for printing. Then porous $\beta$-TCP scaffolds were sintered at $1100{ }^{\circ} \mathrm{C}$ for $2 \mathrm{~h}$ in order to remove the paraffin and densify the scaffold. Anti-tuberculosis drugs, rifampin (RFP) and isoniazid (INH), were loaded on porous $\beta$-TCP scaffolds with PLGA by different steps of centrifuging. Sintered scaffolds had a total porosity of $61.76 \pm 2.53 \%$, with a $400 \mu \mathrm{m}$ macro-porosity of $42.5 \pm 1.23 \%$ and a $2-8 \mu \mathrm{m}$ micro-porosity of $19.26 \pm 2.33 \%$. The maximum compressive strength of this kind of scaffold was $3.31 \pm 0.64 \mathrm{MPa}$. In addition, this porous a-TCP scaffolds showed excellent biocompatibility in vitro, appropriate biodegradation, and controlled drug release, as shown in Fig. 17 [110].

Indirect AM methods are also available for making complex TCP parts. Bose et al. reported a method to prepare porous $\beta$-TCP scaffolds by removing the polymer mold prepared by FDM [111]. The $\beta$-TCP suspension containing food grade TCP, 1-Butanol, D-3021 and binder B-1001 were infiltrated into the mold. Scaffolds were fabricated after drying and sintering of TCP infiltrated molds. The porous $\beta$-TCP scaffolds with a pore size of $305 \mu \mathrm{m}$ and porosity of $29 \%$ showed the maximum compressive strength of around 1.4 MPa. In addition, these porous $\beta$-TCP scaffolds showed excellent biocompatibility by in vitro characterization using osteoblast cell line for 28 days. Limpanuphap et al. also reported an FDM method for creating TCP scaffolds with controlled internal porosity [112]. Molds were printed by two materials including the material for the supporting base and the material for sacrificing to define the structure of the part. Molds were filled with $\beta$-TCP slurry, dried and sintered at $1350{ }^{\circ} \mathrm{C}$ for $2 \mathrm{~h}$. Sintered scaffolds showed an accurate representation of $460 \mu \mathrm{m}$ 
internal pore design, but the mechanical property of this typed scaffolds wasn't mentioned in this study.

Other CaPs: Apart from HA and TCP, there are other $\mathrm{CaP}$ bioceramics with different $\mathrm{Ca}$ to $\mathrm{P}$ ratios. Tetracalcium phosphate (TTCP, $\left.\mathrm{Ca}_{4}\left(\mathrm{PO}_{4}\right)_{2} \mathrm{O}\right)$ has a $\mathrm{Ca}$ to $\mathrm{P}$ ratio of 2, which is the highest in CaPs materials resulting in the lowest degradation among all CaPs. TTCP is a widely used component for bone cement [113]. Dicalcium phosphate (DCP, $\left.\mathrm{CaHPO}_{4}\right)$ is another common calcium phosphate bioceramic with the same amount of $\mathrm{Ca}$ and P. Similar to TTCP, DCP is the main component in "brushite" based cement. Table 7 offers further details of different $\mathrm{CaPs}$ materials [95].

The self-setting reaction of calcium phosphate based cement can be used for AM process. Acid solution can be applied as a binder to print on TTCP or DCP powders for binder jetting process. A mixture of calcium phosphate powder with a $\mathrm{Ca}$ to $\mathrm{P}$ ratio of 1.7 (method A) and TTCP/DCP composite (method B) were printed based on the cement setting reaction using binder jetting technology [107]. Method A had better printing resolution than method B. Scaffolds prepared by method A had 7.4 $\pm 0.7 \mathrm{MPa}$ of compressive strength and $56 \%$ porosity. Scaffolds prepared by method B had only $1.2 \pm 0.2 \mathrm{MPa}$ and $60 \%$ porosity. Klammert et al. also reported a binder jetting method using the reaction between TCP and phosphoric acid to create brushite implants for cranial and maxillofacial defects fixations [114]. Then brushite implants were hydrothermally converted to monetite. Final parts were made with high resolution and accuracy, as shown in Fig. 18. In addition, implants showed a $3.9 \pm 0.5 \mathrm{MPa}$ of point bending strength with a $34.6 \%$ porosity. In another study, brushite and monetite implants were prepared using the similar method for in vivo characterizations [115]. The microporosity of monetite and brushite was $44 \%$ and $38 \%$ and the specific surface area was 6.09 and $3.60 \mathrm{~m}^{2} / \mathrm{g}$, respectively. Intramuscular implantation of both scaffolds demonstrated their excellent osteoinductivity by histomorphometric data from biological stainings. Brushite and monetite scaffolds prepared using this method can be used as the matrix for drugs and angiogenic factors release [116,117]. Scaffolds can absorb vancomycin hydrochloride, ofloxacin, and tetracycline hydrochloride and release them in a controlled behavior by post treatment with PLA/PGA polymer solutions [117]. In addition, in vivo characterization showed enhanced angiogenesis with the presence of copper and Vascular endothelial growth factor (VEGF) [116].

3.1.2. Bioglass-Bioglass is a type of bioactive ceramic material containing $\mathrm{P}_{2} \mathrm{O}_{5}, \mathrm{SiO}_{2}$, $\mathrm{CaO}, \mathrm{Na}_{2} \mathrm{O}$ and $\mathrm{CaO}$ in different ratios. Some commercially available bioglass compositions include bioglass 45S5, which binds to bone and soft tissues, and bioglass 5S4.3 (Ceravital), which binds to only bone tissues. The major advantage of bioglass is its excellent bioactivity. 45S5 bioglass is a widely used commercial bioglass for bone tissue engineering, which has been demonstrated to be beneficial to osteogenesis in vitro and in vivo $[118,119]$. However, due to its poor mechanical properties, it is not suitable for load-bearing applications.

Eqtesadi et al. reported a material extrusion process using a water-based bioglass 45S5 suspension containing poly (methyl vinyl ether) and carboxymethyl cellulose (CMC) additives for controlling rheological property of the material suspension [120]. The addition 
of CMC enabled tuning the rheological properties of ink suspension to meet the stringent requirements of robocasting. This approach was used to study effects of sintering temperatures on compressive strength, density, and microstructure of scaffolds [121]. The compressive strength and density increased with the increasing of sintering temperature. A maximum compressive strength of around $13 \mathrm{MPa}$ was reported for scaffolds sintered at $1000{ }^{\circ} \mathrm{C}$ for $1 \mathrm{~h}$, which had a total porosity of $60 \%$ and pre-designed macroporosity of $50 \%$. Lusquiños et al. reported a selective laser sintering method using bioglass 45S5 and S520 for making implants [122]. Since the melting point of bioglass was lower than calcium phosphates, the powder bed fusion of bioglass was much easier than CaPs ceramics. Crack free three-dimensional geometries were prepared with different laser processing parameters. The bioactivity of SLS processed bioglass was also unchanged by showing the apatite formation.

Tesavibul et al. a vat polymerization method using $43 \%$ of $45 \mathrm{~S} 5$ bioglass and acrylate-based photoactive polymer for of making porous cellular structures [123]. Bioglass based cellular structure with tailored porosity could be prepared to mimic the structure of the trabecular bone. The cellular constructs were featured with a porosity of $50 \%$, a biaxial strength of 40 MPa and a compressive strength of $0.3 \mathrm{MPa}$. This method showed the possibility of using vat polymerization for manufacturing bioglass materials. A more recent study increased the 45S5 bioglass loading to 70\% percent using digital light processing (DLP) based vat polymerization setup to improve the mechanical property of the final part [124]. Final scaffolds were featured with a density of about $2.7 \mathrm{~g} / \mathrm{cm}^{3}$, a high biaxial bending strength of $124 \mathrm{MPa}$, a Weibull modulus of 8.6 and a characteristic strength of $131 \mathrm{MPa}$. The high density and excellent mechanical properties of final scaffolds showed the potential using this method for processing load bearing bioactive glass scaffolds. Binder jetting technology of bioglass was also reported for making multifunctional scaffolds using mesoporous bioactive glass (MBG) and polyvinyl alcohol (PVA) binder for controlled release of the drug [125]. In this study, four different pore sizes, $1307 \pm 40,1001 \pm 48,624 \pm 40 \mu \mathrm{m}$, and $200 \mu \mathrm{m}$, as well as two different pore geometry, square and parallelogram shape, were used for preparing 3D printed MBG scaffolds. Final scaffolds for further characterizations had a square pore morphology, the pore size of $1001 \mu \mathrm{m}$, the porosity of $60.4 \%$, compressive strength of 16.10 $\pm 1.53 \mathrm{MPa}$ and a modulus of $155.13 \pm 14.89 \mathrm{MPa}$. Dexamethasone was loaded on scaffolds and released in a sustained behavior till day 10, as shown in Fig. 19 [125]. In addition, scaffolds showed lower cell proliferation, but higher alkaline phosphatase (ALP) expression than the control. This method provides a new way to make scaffolds with controlled pore structure, high strength and low brittleness without a second high-temperature sintering.

Bioactive glass 13-93 is a new generation of bioglass containing a higher silicate percentage than 45S5 glass, which has been proved to have superior mechanical properties, such as compressive strength and elastic modulus, and better osteogenesis in vitro and in vivo [126128]. Kolan et al. reported a powder bed fusion method using $45 \mathrm{~S} 5$ glass as matrix and stearic acid as the binder to facilitate laser sintering [129]. This method was flexible for making scaffolds with different pore size with no crack formation. The maximum compressive strength reported in this study was $20.4 \pm 2.2 \mathrm{MPa}$ featured with $50.3 \pm 0.8$ porosity. In addition, scaffolds prepared using this method showed excellent bioactivity by showing apatite formation in SBF and high cell proliferation and adhesion using MLO-A5 
cells. A similar method was also used for making 13-93 and 13-93B3 bioactive glasses, in which silicate was replaced with borate for faster degradation [130]. Cubic, spherical, X, diamond, and gyroid pore architectures were used for making porous bioglass scaffolds. In vitro characterization was conducted using a mouse osteoblast cell line. Results showed gyroid and diamond architectures had better cell proliferation compared to other designs. It provided an option to manufacture scaffolds with complex design, large surface area and curvature for improving cell adhesion and growth, as shown in Fig. 20 [130].

3.1.3. Ceramic composites-Instead of single ceramic composition, composites have also been used for different biomedical applications. Different CaPs were combined over the years for scaffold fabrication. HA has excellent biocompatibility, but the biodegradation rate is too low. Hence it is usually mixed with TCP, which is called biphasic calcium phosphate (BCP), for tailoring its biodegradability. Detsch et al. reported a binder jetting method using the $\mathrm{BCP}$ powder for making bone tissue engineering scaffolds [131]. The BCP powder used in this study was spherical with $60 \mathrm{wt} \%$ of HA and $40 \mathrm{wt} \%$ of TCP. The final scaffolds were featured with $53.1 \pm 1.5 \%$ open porosity, but the mechanical property of the scaffold was not mentioned. In addition, scaffolds were tested using monocytic cell line RAW 264.7 for analyzing their effects on osteoclastic differentiation. The final BCP scaffolds showed excellent surface properties for osteoclastic activation. Castilho et al. also reported binder jetting method to prepare porous scaffolds for bone defects fixation [25]. BCP powder with different HA to TCP ratios was used to optimize the printing process. Phosphoric acid was used as the binder to cure scaffolds by the hydraulic setting reaction. Scaffolds prepared using this method had a minimal macropore size of $300 \mu \mathrm{m}$ and a dimension accuracy of $>96.5 \%$. Results showed that a HA to TCP of 1.83 was the best by showing a compressive strength of around $0.42 \mathrm{MPa}$ and a porosity of $66.98 \pm 0.81 \%$ for sintered scaffolds and an increase to around 3.36 MPa after the post-treatment with PLGA. The toughness of scaffolds was quadrupled after the PLGA infiltration. In addition, scaffolds showed enhanced cell viability using osteoblastic cells MG63 compared to pure TCP control. Other CaPs can also be combined as composites to make up for each other's disadvantages. Khalyfa et al. reported a binder jetting method using a powder mixture of TTCP and $\beta$-TCP for making bone tissue engineering scaffolds [132]. TTCP was used as reacting agent with an aqueous citric acid binder. $\beta$-TCP was used as biodegradable filler. Complex internal structures such as scaffolds and cranial segments were fabricated using this method. Scaffolds showed a porosity of $38 \%$ and a compressive strength of $0.7 \mathrm{MPa}$ and an increase to $76.1 \mathrm{MPa}$ after the post treatment using dianhydro-D-glucitol [bis(dilactoylmethacrylate)]. In addition, the biocompatibility of scaffolds was excellent by in vitro characterization using MC3T3-E1 cells.

CaPs and bioglass can also be combined for additive manufacture. Bergmann et al. reported a binder jetting method using $\beta$-TCP and a bioactive glass similar to the 45S5 Henchglass (BGH) for implants fabrication [133]. A mixture of $40 \mathrm{wt} \%$ of $\beta$-TCP and $60 \mathrm{wt} \%$ of BGH glass was used as the matrix powder. The phosphoric acid solution was used as a binder. The four-point bending strength of the printed scaffolds was $14.9 \pm 3.6 \mathrm{MPa}$ after sintering at $1000^{\circ} \mathrm{C}$. Suwanprateeb et al. also reported a CaPs/bioglass composite using apatitewollastonite glass (A-W glass) and HA for implants fabrication using the binder jetting 
method [134]. Sintering scaffolds at $1300{ }^{\circ} \mathrm{C}$ for $3 \mathrm{~h}$ resulted in the lowest porosity of 2.50 $\pm 0.12 \%$ and the highest flexural strength of $76.82 \pm 4.35 \mathrm{MPa}$. In addition, a biocompatibility test in SBF and osteoblast cell culture were conducted on the composite scaffolds, which showed an excellent bioactivity of composite scaffolds.

Several ceramic additives such as strontia $(\mathrm{SrO})$, magnesia $(\mathrm{MgO})$, zinc oxide $(\mathrm{ZnO})$ and silica $\left(\mathrm{SiO}_{2}\right)$, have been used recently to improve the density, phase stability, and biological performances of CaPs scaffolds [135,136,42,97]. An indirect FDM method was used to prepare $\beta$-TCP/MgO and $\beta$-TCP/ZnO porous composite scaffolds [137]. Results indicated that $\mathrm{MgO}$ and $\mathrm{ZnO}$ were able to prevent $\alpha$-TCP phase formation and improve the total density of final scaffolds. Almost $11.5 \%$ density improvement was found for porous $\mathrm{MgO}$ doped TCP scaffolds after sintering at $1250{ }^{\circ} \mathrm{C}$ compared with pure $\beta$-TCP. The compressive strength of $\beta-\mathrm{TCP} / \mathrm{MgO}$ scaffolds was $3.8 \mathrm{MPa}$. Binder jetting technology was used for $3 \mathrm{D}$ printing of interconnected porous scaffolds with different pore size [42]. Binary dopants of $\mathrm{SiO}_{2}$ and $\mathrm{ZnO}$ were used with $\beta$-TCP for the printing. A significant increase of density and compressive strength was found in scaffolds with dopants due to effects of dopant substitution during sintering, as shown in Fig. 21. In addition, scaffolds with dopants showed better bioactivity in vitro using osteoblast cells compared to control $\beta$-TCP [42]. Other dopants such as $\mathrm{SrO}$ and $\mathrm{MgO}$ were also studied with $\beta$-TCP for making interconnected scaffolds via the same additive manufacturing method $[44,138]$. The addition of these two dopants prevented $\beta$ to a phase transition and improved the compressive strength of scaffolds. In vivo characterizations in rat femur model also showed an enhanced osteogenesis due to the introduction of these two dopants, as shown in Fig. 22 [44].

\subsection{Metallic biomaterials}

Currently, many biomedical devices are manufactured using metallic biomaterials. Utilization of different additive manufacturing techniques and the influence of various processing parameters are discussed in this section. The two main metallic materials systems- Titanium based materials and Cobalt based materials are discussed in this section. The section on titanium is further divided into additive manufacturing of single material Titanium based structures for biomedical applications and titanium metal matrix composites. Due to their importance, a separate sub-section is dedicated to the titanium metal matrix composites with calcium phosphate as the ceramic additive. The section on Cobalt alloys also includes information on additive manufacturing of simple cobalt based structures and cobalt based metal matrix composites for biomedical applications.

3.2.1. Ti-based metallic biomaterials-Titanium and its alloys are a very popular material for biomedical applications. Ti-based materials have shown excellent biocompatibility. They also have excellent resistance to fatigue loading and corrosion. Further, an inherent high strength to weight ratio makes parts made out of Ti-based materials lighter than other metallic materials. However, Ti-based materials have some drawbacks. The tribological performance of Ti-based materials is rather poor. This problem stems from the mechanical inadequacy of the Ti-based materials. Most applications of Ti-based materials are for load-bearing implants such as total hip or total knee replacement. In applications like these, a satisfactory bonding between the human tissue and the new implant 
is a must. It has been found that a hard tissue layer surrounds the Ti implant over time. Thus, even though Ti-based materials are an established materials system for biomedical implants applications, there are still shortcomings that need to be addressed. Additive manufacturing techniques can offer some solutions to these problems. Such solutions range from the surface modification of the Ti-based materials to improve its wear performance to fabricating porous or structurally graded parts to enhance osseointegration.

Fabrication of 3D structures from Ti-based metallic biomaterials: Laser additive manufacturing of Ti64 alloy was shown by Kobryn et al. [56]. The researchers investigated the range of microstructures that could be obtained as a result of laser additive manufacturing of the $\beta$ alloy. The LAM processing was done with a Nd:YAG and with a $\mathrm{CO}_{2}$ laser process. Columnar grains were observed in the microstructure. For Nd:YAG laser processing, columnar grains were found to grow at a fixed acute angle with respect to the Ti64 substrate whereas the columnar grains of the alloy deposited with a $\mathrm{CO}_{2}$ laser source grew perpendicular to the substrate. The microstructures showed fine Widmanstatten patterns. Fine equiaxed a phase particles were found in the individual columnar grains and also along the individual grain boundaries during Nd:YAG source deposits. With the $\mathrm{CO}_{2}$ source deposits, grains were coarser. There was also a visible thermal effect of the depositing layers on the microstructure of the previously deposited layers. In this case, banding of the microstructure or sudden change in the number of equiaxed a phase particles was observed. The grain width of the $\beta$ phase was affected due to laser additive manufacturing, with the average grain width ranging from $94 \mu \mathrm{m}$ to $165 \mu \mathrm{m}$. The grain width was also found to increase with the incident laser energy due to slower solidification/cooling rate. The microstructural features are shown in Fig. 23 [56].

Capabilities of direct laser fabrication (DLF) were also demonstrated by Wu et al. [139]. A $20 \mathrm{~mm} \times 20 \mathrm{~mm}$ section tube was processed with a dimensional accuracy of $\pm 0.05 \mathrm{~mm}$. A finely focused laser beam produced a wall thickness of approximately $1.3 \mathrm{~mm}$ whereas a defocused beam had a fuzzy wall thickness of approximately $3.5 \mathrm{~mm}$. It was also shown that overhang structures with up to 30 degrees of inclination can be successfully processed. The researchers also reported occasional porosity that was round and most likely caused either by trapped gas from the feed powder or from the positively pressurized argon carrier gas. The effect of power on the porosity was also investigated. At a power level of $264 \mathrm{~W}$, the microstructure was completely dense. Some porosity, mainly along the $\beta$ phase grains was found at a power level of $390 \mathrm{~W}$. At a lower power of $180 \mathrm{~W}$, microstructure showed connected porosity with pore diameters as high as $300 \mu \mathrm{m}$. Similarly, the laser scan speed also had a significant effect on the microstructural evolution where high scan speed generally yielded a coarse microstructure.

Kelly et al. studied microstructural and thermal modeling on laser processed Ti64 alloy using Laser Additive Manufacturing (LAM) setup of AeroMet Corporation [140]. Similar to the earlier findings, researchers found laths of $\alpha$-Ti that were bordered with the $\beta$-Ti grains. Within each of these bands, there were dense colonies of equiaxed a-Ti grains. Vicker's microhardness measurement showed the hardness at approximately $350 \mathrm{HV}$ for the LAM deposited Ti64 alloy. Porous Ti structures were demonstrated by Balla et al. and Xue et al. using LENS ${ }^{\mathrm{TM}}[61,141]$. As opposed to complete feedstock powder melting and 
solidification in earlier attempts of processing Ti-based materials, in this research, the key to forming porous Ti components was partial melting of the feedstock powder. In this principle, only the outside surfaces of the powder were melted and the internal cores remained intact, forming porous structures as shown in Fig. 23 [61]. These melted surfaces caused the bonding of particles together along with residual porosity. In addition, final parts showed similar Young's Modulus compared to the natural bone. In vitro characterizations also demonstrated that porous Ti manufactured by LENS ${ }^{\text {TM }}$ had improved biological properties compared to bare Ti plate as shown in Fig. 23 [141].

Heinl et al. demonstrated the fabrication of cellular Ti structures using Selective Electron Beam Melting process [142]. Structures were fabricated out of Ti64 powder at a vacuum pressure of $10^{-4}$ to $10^{-5} \mathrm{mbar}$, thus preventing any possible contamination due to oxygen or nitrogen. The three attempts made at fabricating interconnected porous structures were successful with samples of three different porosities as $25 \%, 38 \%$, and $60 \%$ by volume. Harrysson et al. demonstrated EBM manufacture of Ti64 structures with different densities ranging from $3.8 \%$ to $40 \%$, and subjected them to compression and three-points bend flexural testing [143]. The compressive strength was found to decrease with increasing the cell size or increasing porosity in the structure. Some examples of these EBM processed structures are shown in Fig. 24 [143]. Structures with a cell size of $3 \mathrm{~mm}$ and relative density of 0.41 had a compressive strength of $85.72 \mathrm{MPa}$ whereas the structure with a cell size of 12 $\mathrm{mm}$ and relative density of 0.04 had a compressive strength of $0.84 \mathrm{MPa}$.

Murr et al. compared microstructural and mechanical properties of EBM fabricated Ti64 components to their wrought counterparts [144]. The EBM manufactured samples showed Windmanstatten microstructure. The average thickness of the columnar a phase was $3.2 \mu \mathrm{m}$ in the top region of the build whereas it was $1.6 \mu \mathrm{m}$ at the bottom region of the build. From TEM analysis, it was found that the wrought alloy had lesser dislocation density than the EBM fabricated alloy. Ti64 alloy powder used for the EBM fabrication had an average hardness of 5.0 GPa. It was found that the upper regions of the EBM fabricated Ti64 alloy part had coarse a-plate grains and thus lesser hardness of 3.6 GPa whereas the lower regions had finer a-plate grains and higher hardness between $3.9 \mathrm{GPa}$ and 4.6 GPa. However, the EBM manufactured samples showed $23 \%$ to $92 \%$ higher elongation than average elongation for the high strength forged Ti64 alloy components. EBM technique was also used to fabricate open cellular foams using Ti64 alloy. The foams that were manufactured had a porosity ranging from $55 \%$ to $89 \%$, and the cell walls or the struts of these foams had a hardness in the range of 4.1-4.9 GPa. These hardness numbers were higher than the hardness that was recorded in the denser Ti64 components, also fabricated by EBM technique. This could be due to the faster cooling rate in thin struts/cell walls of the foam structures as compared to the overlapping layers in the denser parts. The denser parts showed an acicular/lamellar Windmanstatten structure with a phase whereas the cell walls of the foams showed predominantly a' phase or the martensitic a phase. Authors also demonstrated the capability and scalability of the process by fabricating a component of 2 $\mathrm{cm}$ diameter that mimicked the natural bone. This Ti64 component had lesser porosity on the outside (like the cortical bone) and higher porosity on the inside (like the cancellous or trabecular bone). Properties and mechanical performance of similar EBM fabricated Ti64 structures were also studied later on by other research groups $[145,146]$. 
Selective laser sintering (SLS) or selective laser melting (SLM) has also been utilized for processing dense and porous Ti-based materials towards biomedical applications. Das et al. demonstrated processing of net shapes of Ti64 alloy using SLS followed by hot isostatic pressing (HIP) [147]. Ti64 powder was first selectively sintered with a laser source to form a 92\% dense component. Post processing with HIP further enhanced the density of the part. Since the parts fabricated this way were near net shaped, an additional machining process was needed. It was reported that microstructures of SLS/HIP processed parts varied from outer skin to the inside. The skin or the outer walls exhibited a fine Widmanstatten structure with high aspect ratio of the lamellar phase whereas the bulk microstructure consisted of coarser grains. The difference in microstructure was attributed to surface melting of powders resulting in a thermal gradient along the cross-section. To demonstrate the practicality of the SLS/HIP process, a sub-scaled version of a housing component of the AIM-9 Sidewinder missile was manufactured. This component had a fully dense microstructure. Sintering of Ti powder was also demonstrated by Fisher et al. [148]. Authors proposed a process map for the laser sintering of Ti powder with Nd:YAG pulsed laser source.

Hollander et al. produced Ti64 components with SLS or DLF (Direct Laser Forming) process and characterized those parts in vitro with human primary osteoblast cells [149]. To validate the feasibility of SLS/DLF part fabrication, authors processed a replica of a human vertebra, as shown in Fig. 24 [149]. The unfinished surfaces of the DLF parts appeared granulated and finishing operation had to be performed. Porous components had a nominal pore size between $500 \mu \mathrm{m}$ and $700 \mu \mathrm{m}$ and general pore shapes were cylindrical in nature. In terms of mechanical properties, the SLS/DLF parts had a tensile strength of $1200 \mathrm{MPa}$ and a $6.5 \%$ elongation. After post-process annealing heat treatment, elongation at failure increased twofold to $13.0 \%$ and reduced the tensile strength to $1042 \mathrm{MPa}$. In vitro results confirmed that samples were non-toxic. Authors performed in vitro cell culture on porous samples with average pore diameters of $500 \mu \mathrm{m}, 700 \mu \mathrm{m}$, and $1000 \mu \mathrm{m}$. After 14 days, samples with 500 $\mu \mathrm{m}$ pore diameter showed the best cell-materials interactions. Kruth et al. reported a comparative wear behavior of different materials processed by SLS/SLM technique. Different materials were subjected to fretting wear tests under $2 \mathrm{~N}$ and $6 \mathrm{~N}$ load and their wear damages were recorded in terms of $\mathrm{mm}^{3}$ of material lost for 10,000 cycles [150]. At 2 $\mathrm{N}$ load, Ti64 alloy had a wear volume of $7.362 \times 10^{3} \mathrm{~mm}^{3}$, and at $6 \mathrm{~N}$ load, it was $9.337 \times$ $10^{3} \mathrm{~mm}^{3}$. For the CoCrMo alloy, the damages at $2 \mathrm{~N}$ and $6 \mathrm{~N}$ loads were $1.474 \times 10^{3} \mathrm{~mm}^{3}$ and $2.252 \times 10^{3} \mathrm{~mm}^{3}$, respectively.

Shishkovsky et al. evaluated mechanical properties, corrosion behavior and microstructural features of SLS/SLM manufactured porous implants formed from a Ti and a NiTi shape memory alloy [151]. Mechanical characterization showed that samples failed by brittle to brittle-ductile fracture. After 3 months in white laboratory rats, the SLS processed NiTi samples were completely integrated with the organotypic structures. There was no observation of either localized or general corrosion. Nitinol implants did not show any adverse tissue reaction. Fig. 25 shows the SLS fabricated NiTi structures and the bone integration with the titanium and nitinol implant after three months [151]. Authors also carried out SLS processing of Ti and nitinol powders with hydroxyapatite (HA). The presence of HA was found to intensify cell proliferation. Porous Ti scaffolds were also produced using selective laser melting [152]. In this work, however, there was no designed 
porosity in the initial structure of the material. Porosity was created by mixing $\mathrm{TiH}_{2}$ and $\mathrm{Ti}$, where during laser processing, the heat input decomposed $\mathrm{TiH}_{2}$ while releasing hydrogen gas. The trapped hydrogen gas then acted as a porogen or a pore forming agent. Using a $\mathrm{TiH}_{2}$-Ti mixture containing $40 \mathrm{wt} \%$ of $\mathrm{Ti}$, a high degree of porosity of up to $70 \%$ was achieved along with pore size of $200-500 \mu \mathrm{m}$ at a laser power of $1000 \mathrm{~W}$. Porosity increased with an increase in $\mathrm{TiH}_{2}$ content. Porous Ti structures, similar to the cancellous bone, were also reported using SLM processing [153]. Samples were fabricated with a porosity between $75 \%$ and 55\% and had compressive strengths between 35 and $120 \mathrm{MPa}$ depending on the porosity. General pore size was between $400 \mu \mathrm{m}$ and $800 \mu \mathrm{m}$. New bone formation was examined in white rabbits and no adverse reactions such as inflammation or any type of foreign body reaction were observed in the animals after surgery. Within three weeks, new bone formation was observed at the outer periphery and in the center of the chemical and heat treated implants. Also, most new bone that was formed seemed to be bonded directly to the chemically treated layers, which was different to the untreated implants. The findings can be seen in Fig. 25 [153]. Mechanical properties and their relationship to the processing parameters were also investigated for SLS/SLM processed Ti alloy parts $[154,155]$.

Another method of processing porous Ti-based structures is 3D fiber deposition that was originally designed for 3D fabrication of polymeric components. Li et al. reported the bonematerial interaction aspect in the scaffolds produced by 3D fiber deposition [156]. Ti64 alloy powder and $0.5 \%$ aqueous methylcellulose solution was used to create a slurry that was forced through a syringe in a Bioplotter machine. Such layer-by-layer deposition forms a complete 3D scaffold that was later sintered at $1200{ }^{\circ} \mathrm{C}$ for $2 \mathrm{~h}$. These samples had porosities ranging from $39 \%$ to $68 \%$ with differing rectangular and square shaped pore sizes ranging from $200 \mu \mathrm{m}$ to $800 \mu \mathrm{m}$. Samples were then inserted into lumbar goat spines for in vivo analysis. Animals were sacrificed after 12 weeks. There was no evidence of any toxicity or inflammation. The amount of new bone formation increased with increasing the pore size, and the overall porosity of the implant. Porous Ti scaffolds were also fabricated by Ryan et al. using a wax model printed using a Thermojet 3D printer [157]. A slurry of Ti powder and ethylene glycol was poured into the wax mold and dried. After removal of the wax, the Ti structure was sintered between $1100{ }^{\circ} \mathrm{C}$ and $1300{ }^{\circ} \mathrm{C}$ under vacuum. SAOS-2 pre-osteoblast cells were cultured on these sintered Ti scaffolds for over three weeks without any concerns of toxicity. These scaffolds had a porosity of $45 \%$ with an average pore size of $465 \pm 170 \mu \mathrm{m}$. Scaffolds were also fabricated with pore sizes of $200 \mu \mathrm{m}, 300 \mu \mathrm{m}$ and 400 $\mu \mathrm{m}$ and porosity as high as $66.9 \%$.

Metal matrix composites: Unlike work with pure metals and alloys, AM of metal matrix composites (MMCs) are not that common. Over the years, researchers have attempted to use laser cladding or laser melt injection methods to form a composite microstructure of the reinforcing material in a metal matrix $[58,60,158,159]$. Apart from those approaches, other methods of direct energy additive manufacturing such as direct laser deposition and LENS ${ }^{\mathrm{TM}}$ have also been utilized for processing of different ceramic based materials with Ti matrix. In situ reinforcement of Ti64 was done by TiB-TiN using LENS ${ }^{\mathrm{TM}}$, and its tribological properties, in vitro biocompatibility were also measured [160,161]. During LENS ${ }^{\mathrm{TM}}$ processing, Ti64 powder was premixed with $\mathrm{BN}$ powder and the resultant materials formed 
$\mathrm{TiB}$ and TiN as well as alpha and beta phases of Ti as seen in Fig. 26 [160]. Hardness values increased to $>850 \mathrm{HV}$ due to the addition of $15 \%$ of $\mathrm{BN}$ in the premixed powder. Wear resistance of this material was also found to be better than the CoCrMo alloy, the gold standard for load-bearing implants. After 11 days of in vitro cell culture with MG63 cells, there was no difference between the cell adherence on the coatings with $\mathrm{BN}$ and untreated $\mathrm{Ti}$ substrate. Similar LENS ${ }^{\mathrm{TM}}$ processing of Ti was done using TiC powders [162]. LENS ${ }^{\mathrm{TM}}$ based fabrication of compositionally graded materials was also achieved for TiC-Ti system in which $\mathrm{TiC}$ was simultaneously fed with $\mathrm{Ti}$ [162]. The proportion of $\mathrm{TiC}$ was gradually increased from $0 \%$ to $95 \%$. This had an immediate effect on the phase and microstructural evolution. With increasing $\mathrm{TiC}$, more beta titanium phase formed. $\mathrm{TiC}$ was also retained in the microstructure as a unmelted phase. A hardness of $2250 \mathrm{HV}$ was achieved in these compositionally graded structures.

$\mathrm{Ti}^{-\mathrm{TiO}_{2}}$ compositionally graded structures were processed using LENS ${ }^{\mathrm{TM}}$ [63]. The amount of $\mathrm{TiO}_{2}$ was increased from $50 \%$ to $90 \%$. A hardness of $1100 \mathrm{HV}$ was achieved due to the addition of $50 \% \mathrm{TiO}_{2}$ in Ti. SiC has also been reinforced in Ti using LENS ${ }^{\mathrm{TM}}[163,164]$. Tribological performance of the Ti substrate was improved by more than an order of magnitude with hardness reaching up to $1000 \mathrm{HV}$. In a rather unusual approach of formation of in situ ceramic phase, Zhang et al. demonstrated that nitrides of Ti and Si can be deposited on Ti substrates by introducing nitrogen in the oxygen free-argon rich chamber during LENS ${ }^{\mathrm{TM}}$ operation [165]. Thereafter, a mixture of Ti and Si powders were fed and coatings were deposited on the surface of Ti. The resultant structures were a composite of TiN and $\mathrm{Si}_{3} \mathrm{~N}_{4}$ dendrites in the matrix of alpha Ti. The hardness of these in situ formed ceramic coatings was in the range of 1300-2100 HV and wear damage was reduced by as much as 200 times compared to Ti substrate.

Metal matrix composites with calcium phosphate: Bone is a natural calcium phosphate $(\mathrm{CaP})$ based ceramic. The addition of $\mathrm{CaP}$ to metal matrix is exciting due to the enhanced biocompatibility of the composite surface. Direct pulsed laser irradiation of a mixture of brushite and calcite was earlier demonstrated on steel [166]. However, it was only in 2002 that $\mathrm{CaP}$ based materials were directly processed via an additive manufacturing route on Tibased materials. Laser alloying or laser cladding of hydroxyapatite was done on Ti64 alloy [167]. Laser cladding of Ti with calcium phosphate was also done in multiple steps by Zheng et al. [168]. Laser cladding was first done with $80 \% \mathrm{Ti}-20 \% \mathrm{CaP}$ followed by $60 \% \mathrm{Ti}-40 \% \mathrm{CaP}$ and finally $100 \% \mathrm{CaP}$. This multi-step laser cladding also helped to reduce thermal stresses during laser cladding. A maximum hardness of up to $1600 \mathrm{HV}$ was obtained. However, a variety of phase formation has been reported such as alpha and beta-tricalcium phosphate, $\mathrm{TiO}_{2}$ and $\mathrm{CaO}$. Rat cell line ROS 17/28 was used to study cell proliferation on these bioceramic coatings, and it was found that the bioceramic coating had more favorable cell response compared to the untreated Ti64 substrate.

LENS $^{\text {TM }}$ based additive manufacturing technique was used for fabricating CaP coatings on titanium $[169,170]$. These coatings were deposited at laser powers of $400 \mathrm{~W}$ and $500 \mathrm{~W}$. At a power of $400 \mathrm{~W}$, a coating of thickness $250 \mu \mathrm{m}$ was achieved whereas at $500 \mathrm{~W}$, the coating was $400 \mu \mathrm{m}$. It was also found that lower feed rates of the powder and high laser power of $500 \mathrm{~W}$, the coating thickness significantly reduced to $100 \mu \mathrm{m}$. The interface 
between the TCP coating and the Ti substrate showed columnar Ti grains and TCP at the grain boundaries. A representative image of this microstructure is shown in Fig. 26 [169]. At the uppermost regions of the coating, grains were primarily equiaxed. The volume fraction of TCP was highest at the uppermost region. The increase in the concentration of TCP was gradual from the interface, which was evident from the hardness of the coatings as well. A maximum hardness of up to $1100 \mathrm{HV}$ was achieved at the top surface with an average of 900 HV. This was more than four times the hardness of the Ti substrate. To understand the activity of human osteoblast cells on LENS ${ }^{\mathrm{TM}}$ processed TCP coatings, in vitro cell culture was done. After just 5 days of incubation, more cell attachment was found on TCP coatings than the control Ti surface. After 11 days, layers of cells were formed on the TCP coating. This increase in the cell attachment and proliferation was further confirmed by MTT assay, as shown in Fig. 26.

Surface alloying of Ti-based materials: Surface alloying is basically a surface modification of the parent material with another metallic material to improve surface properties. It was suggested that using high power density and solidification rates of laser energy, surfaces could be modified and non-equilibrium phases could be retained. This also gave rise to unique microstructures such as extended solid solutions, metastable crystalline phases, and metallic glasses [171]. Laser assisted surface alloying has been extended towards many metallic systems. Some of these investigations were done using preplaced powders on the surface and then remelting the powder with a laser to achieve bonding and solidification of the powder.

Surface alloying of metals of biomedical importance was discussed by Walker et al. [172]. In this early work, commercially pure Ti (cp-Ti) and Ti64 alloy were surface alloyed with carbon, and cp-Ti and Ti15Mo alloy were alloyed with nitrogen. Compositionally graded structures of Ti64 alloy were formed with CoCrMo alloy [62,64]. These compositionally graded structures were fabricated using LENS ${ }^{\mathrm{TM}}$ and their microstructures are shown in Fig. 27 [64]. These structures were also structurally graded. The Ti64 alloy part of the structure had $\sim 30 \%$ porosity to promote osseointegration. After six LENS ${ }^{\mathrm{TM}}$ deposited layers of the porous Ti alloy, the structure transitioned to $100 \%$ dense Ti64. Thereafter, the $100 \%$ dense structure transitioned from $100 \%$ Ti64 to CoCrMo alloy. In vitro wear testing of these structures was done in simulated body fluid (SBF). The concentration of Co ions leached after wear testing for $3000 \mathrm{~m}$ was also investigated and was found to decrease with increasing CoCrMo concentration from 50\% CoCrMo to $100 \%$ CoCrMo. The wear rates also dropped in a similar fashion.

Apart from these metallic materials and metal-ceramic combinations, other non-traditional metallic materials have also been investigated for biomedical applications. LENS ${ }^{\mathrm{TM}}$ was utilized for processing of tantalum coatings on Ti substrates [173]. Tantalum, despite being a challenging material to process owing to its high melting temperature and poor thermal conductivity, was successfully processed and hardness of up to $350 \mathrm{HV}$ was achieved. The microstructure of this coating is shown in Fig. 28 [173]. Biocompatibility of these coatings was investigated using human $\mathrm{hFOB}$ cells in vitro. Quantification of the cell proliferation using MTT assay showed higher proliferation of cells on the Ta coatings than Ti substrate. 
Furthermore, wear properties of the LENS ${ }^{\mathrm{TM}}$ processed Ta coatings was almost an order of magnitude lower than the Ti substrates [174]. These results can be seen in Fig. 28 [174].

Roy et al. attempted doping of Ta with $\mathrm{MgO}$ and LENS ${ }^{\mathrm{TM}}$ processing of the doped $\mathrm{Ta}$ on $\mathrm{Ti}$ substrates [175]. It was found after 3 and 7 days of human hFOB culture in vitro that the cell attachment and proliferation on the $\mathrm{MgO}$ doped LENS ${ }^{\mathrm{TM}}$ processed Ta coatings was higher than the undoped LENS ${ }^{\text {TM }}$ Ta coatings. The bioactivity of the Ta coatings was thus further amplified by selective doping with $\mathrm{MgO}$, as shown in Fig. 28 [173,174]. Vrancken et al. reported the SLM processing of Ti64 alloy composite with $10 \mathrm{wt} \%$ Mo additions [176]. This mixture was processed at a power of $250 \mathrm{~W}$ with $30 \mu \mathrm{m}$ layers. It was found that after the addition of $10 \mathrm{wt} \%$ of Mo in Ti64 alloy, the very typical columnar $\beta$ phase grains disappeared. There was the presence of unmelted Mo particles, smaller than $10 \mu \mathrm{m}$ in size, in the structure. The Young's modulus of this Ti64-10Mo alloy was lower than the Ti64 alloy. The alloy with Mo also had a lower yield strength of $858 \mathrm{MPa}$ than $1110 \mathrm{MPa}$ for the substrate. However, the elongation of the alloy with $10 \%$ Mo was $20 \%$ compared to $7.3 \%$ for the Ti64 substrate.

In other attempts, selective laser melting (SLM) was utilized to process a porous niobium coating on Ti substrate [177]. Laser power of $160 \mathrm{~W}$ was used for the melting of $\mathrm{Nb}$ on the surface of Ti. The resultant coating had good metallurgical bonding to the Ti substrate as seen from Fig. 29 [177]. Average grain size of $70 \pm 20 \mu \mathrm{m}$ was obtained on the SLM processed porous $\mathrm{Nb}$ coatings on Ti along with hardness of $392 \pm 37 \mathrm{HV}$. Circular and cubic micro-sized pores were formed throughout the melted layers of $\mathrm{Nb}$ coating. Good cell attachment was observed on both the Nb coatings as well as the Ti substrate. After 3 and 7 days of cell culture, CCK-8 assay was used to determine the cell proliferation and no significant difference was seen between the porous $\mathrm{Nb}$ and the Ti samples. However, after 11 days, there was higher cell proliferation on the porous $\mathrm{Nb}$ coatings than on the Ti substrate.

3.2.2. Co-based metallic materials and composites-As with the other materials that were previously discussed for additive manufacturing, the earliest forms of additive manufacturing of Co-based materials involved laser cladding of these alloys on materials like steel for high wear and abrasion resistance properties. In addition to the use of additive manufacturing techniques for improving the surface wear properties, there has a considerable amount of research into the fabrication of three-dimensional bulk components via additive manufacturing. In one of the very early demonstrations by laser cladding, Stellite- $\mathrm{SiC}$ composites were fabricated. Laser cladding was carried out using a $2 \mathrm{~kW} \mathrm{CO}_{2}$ laser [57]. Stellite 6 alloy with different proportions of $\mathrm{SiC}$ were fed into the laser melt pool. The hardness of up to $1390 \mathrm{HV}$ at $19 \mathrm{wt} \% \mathrm{SiC}$ in Stellite 6 was achieved. Similarly, Zhong et al. demonstrated laser clad composites of Stellite 6 alloy and WC. Using dual powder feeders, WC amount was varied in the melt pool from $0 \%$ to $100 \%$ [178]. On similar grounds, Xu et al. formed Stellite-WC composites in the range of 0 to $47 \mathrm{wt} \%$ of WC by multi-layer laser cladding to create functionally graded structures using a $\mathrm{CO}_{2}$ laser and Argon shielding gas [179]. In this approach, the proportion of WC that was feed through the powder delivery system was gradually increased from $0 \mathrm{wt} \%$ in the first layer to $47 \mathrm{wt} \%$ at the end. The microstructure that evolved consisted of a hypoeutectic structure of $(\gamma+(\gamma$ $+\mathrm{WC})$ ) where $\gamma$ was a primarily Co-rich solid solution and $\gamma+\mathrm{WC}$ was the eutectic 
structure. Some unmelted WC particles were also observed and can be seen in Fig. 30 [179]. Along with different carbides of $\mathrm{Cr}_{23} \mathrm{C}_{6}, \mathrm{Co}_{3} \mathrm{~W}_{3} \mathrm{C}$. A layer of Stellite 6 alloy with 26 wt $\%$ WC reached a hardness as high as $800 \mathrm{HV}$.

These demonstrations of additive manufacturing of Co-based materials utilized materials in a coating form or for surface modification. From a biomedical applications point of view, it is important to understand the feasibility of these materials in a bulk form. Janaki Ram et al. demonstrated LENS ${ }^{\mathrm{TM}}$ based additive manufacturing of medical grade CoCrMo alloy [180]. $6 \mathrm{~mm}$ thick samples were processed using LENS ${ }^{\mathrm{TM}} 285 \mathrm{~W}$ laser power. The microstructure of each layer consisted of slightly coarser columnar dendritic phase and very fine equiaxed dendrites. These microstructural variations resulted from the variations in the solidification conditions. The presence of carbide phase in the columnar dendritic and equiaxed dendritic regions was also reported. The carbide phase was present either as a thin continuous network or as particles in the interdendritic regions. The hardness of the LENS ${ }^{\mathrm{TM}}$ deposited CoCrMo alloy was $40 \mathrm{HRC}$ and thus comparable to the hardness of the wrought substrate of $41 \mathrm{HRC}$. The abrasive wear resistance was found to be less than that of the substrate. Authors attribute this to the irregular shaped carbide phase that was basically present as thin and long interconnected particles whereas it was much more regular, spherical and uniformly distributed in the wrought substrate.

Dense and porous Co-based alloys were also manufactured using an E-beam melting technique [181]. Different joint replacement components as well as test specimens fabricated using E-beam process are shown in Fig. 31 [181]. Fully dense components from CoCrMo alloys with a density of $8.4 \mathrm{~g} / \mathrm{cm}^{3}$ were processed. In addition, femoral knee implants and open cellular mesh components were also manufactured using E-beam based additive manufacturing approach. As-fabricated femoral knee implant components were also given the standard ASTM F75 CoCr alloy treatment and were followed by annealing heat treatment. Fully dense components showed a microstructure with arrays of carbides and zigzag carbide phases along with columnar grains. In the horizontal plane for a rectangular block of fully dense E-beam processed CoCrMo alloy, the hardness averaged $4.4 \mathrm{GPa}$ and for a cylindrical sample, it was $4.6 \mathrm{GPa}$. These hardness values are about $30 \%$ lower than the CoCrMo powder used for processing these samples. For the femoral knee component prototype, microstructure consisted of a mixture of columnar carbides and carbide arrays that measured about 2-3 $\mu \mathrm{m}$. Similarly, femoral components that were annealed and polished, the microstructure showed an equiaxed fcc grain structure with prominent annealing twins and finer carbide phases as seen in Fig. 32 [181]. For the reticulated mesh structure of CoCrMo alloy, microstructure of the struts was similar to the microstructure of fully dense block and cylindrical samples. However, the hardness was about $25 \%$ higher than the dense structures and was $6.8 \mathrm{GPa}$ in the horizontal plane of the struts, and 5.6 GPa in the vertical plane.

Jevremovic et al. studied biocompatibility of selective laser melted CoCr alloy for removable dental applications via MTT assay, agar diffusion, and dye exclusion tests [182]. It was found that the total cell number and viability of the cells that were pre-incubated with the samples fabricated by the conventional method of casting and by SLM method were on par with the control sample. The differences between the growth inhibitory effect of the cast and 
SLM fabricated samples in MTT assay were below 30\%. The agar diffusion tests also did not show any deleterious effects. It was concluded that the SLM processed $\mathrm{CoCr}$ alloy was not cytotoxic nor did it release any harmful material that could act against the cells. SLM process was also employed to understand pore formation in CoCrMo alloy [183]. In attempts to build the completely dense sample, researchers found that the SLM technique was capable of making CoCrMo components with a lowest possible porosity of $0.9 \%$ and an average porosity of $5.77 \%$. It was also found that the highest layer thickness while processing led to minimal porosity due to the enhanced thermal conductivity of the material and secondary densification. The mean pore size was $0.759 \mu \mathrm{m}^{2}$ and it remained constant irrespective of the different layer thicknesses and laser power. However, reducing the laser scanning speed increased this mean porosity by as much as $41.17 \%$. Hedberg et al. investigated in vitro biocompatibility of the structures fabricated using SLM process [184]. SLM processing led to a fine grained structure with grain boundaries rich in Mo and depleted in Co. There was also the formation of martensitic $\varepsilon$ or the hcp phase with no carbide formation unlike reported previously by many researchers. In the ion release studies conducted in PBS (phosphate buffer saline) and PBS+BSA (bovine serum albumin), it was found that after $2 \mathrm{~h}$ and $168 \mathrm{~h}$ of release, the SLM fabricated parts released fewer ions as compared to the cast parts. The SLM fabricated parts also showed higher corrosion resistance than cast parts. Researchers have also used SLM process for CoCrMo alloy fabrication towards dental applications [185,186].

In summary, AM of various techniques ranging from powder bed fusion to direct energy deposition have shown the ability to fabricate metallic materials that are used in biomedical applications. In addition, AM approaches are being further explored to solve current shortcomings in the metallic biomedical materials such as early stage material-bone integration or osseointegration of the implant and the reduction of stress shielding in loadbearing implants. AM approaches are also being used in fabricating unique, patient-specific implants that are difficult to manufacture using conventional manufacturing.

\subsection{Additive manufacturing of polymers}

Polymers are first materials used in AM. Apart from the vat polymerization, polymers can also be used in other first generation of AM technologies, such as material extrusion and powder bed fusion. Compared to metals and ceramics, polymers have advantages of high manufacturability due to their low melting points and the flexibility to modify their chemical structures. Apart from those advantages of polymers in AM, there are other properties necessary for polymers to be used in biomedical applications. Biocompatibility is necessary for implants to be compatible with host tissues. In addition, biodegradability is also necessary for polymers to degrade after the tissue self-recovery. Polymers that have those properties are generally called biopolymers. Biopolymers can be broadly classified into two categories: natural and synthetic biopolymers. The following section will discuss the AM of these two types of biopolymers.

3.3.1. Natural biopolymers-Natural biopolymers are biopolymers which can be originally found in nature. Collagen is one popular biopolymer in AM which is often a significant component of extracellular matrix (ECM). There is a significant interest for 
collagen to be used in tissue engineering. Collagen has been widely used as the tissue engineering scaffold material and the drug delivery vehicle because of its excellent biocompatibility [187]. The AM of collagen has also been studied in the past few years. Since collagen can denature under high temperature and hardly form powder bed with good flowability, material extrusion was the major AM technology used to manufacture 3D collagen architectures $[188,189]$. Kim et al. reported a cryogenic plotting system for printing highly porous collagen scaffolds with various porosity for skin tissue repair [188]. The maximum tensile strength achieved using this method was $2.8 \mathrm{MPa}$ with a porosity of 95.3\%. A co-culture of keratinocyte/fibroblast was performed on final scaffolds showing good cell migration and well-differentiated keratinocytes similar to the human skin. Growth factor was also incorporated into 3D printed collagen scaffolds for making artificial neural tissues [189]. VEGF releasing fibrin gel, collagen bottom, murine neural stem cells (C17.2) and collagen top were printed using 3D bio-printer following this sequence. VEGFcontaining fibrin gel promotes migration and proliferation of the C17.2 cells cultured in collagen scaffolds, as shown in Fig. 33 [189].

Indirect AM has also been used to manufacture collagen scaffolds [190]. The negative mold was produced using FDM method. After the collagen casting, negative molds were removed by immersion in ethanol. The final collagen scaffolds were not contaminated or denatured which was confirmed by Fourier Transform Infrared Spectra and Ultraviolet-visual spectroscopy. Liu et al. reported similar indirect AM method for making collagen scaffolds [191]. The final scaffolds had interconnected channels and hierarchical designs with the length scale from $10 \mu \mathrm{m}$ to $400 \mu \mathrm{m}$. In vitro characterizations using human mesenchymal stem cells (hMSCs) showed excellent cell adhesion, proliferation, and migration till day 28. In addition, post-crosslinking treatment improved the dynamic properties of scaffolds.

Gelatin is another popular biopolymer, which is extracted from animal by-products, such as bones, skins, and connective tissues. Gelatin is inexpensive and shows excellent biodegradability, biocompatibility, and non-immunogenicity [187,192,193]. Common uses of gelatin include hydrogel formation and vessels for controlled drugs release [187,194]. Gelatin constructs were printed using material extrusion with hepatocytes under a lateral resolution of $10 \mu \mathrm{m}$ [195]. Gelatin provided a stable structure and nutrient for cells to grow. Hepatocytes distributed using this method on gelatin matrix remained viable for more than two months, which provided a novel method for tissue and organ printing. Gauvin et al. reported a novel projection stereolithography technique for preparing gelatin methacrylate (GelMA) scaffolds [196]. Side group modification of methacrylamide moieties on gelatin made vat polymerization possible for fabricating gelatin scaffolds with complex architecture for tissue engineering. Two different scaffold designs, woodpile and hexagonal, and GelMA percentage, $10 \%$ and $15 \%$, were used in the printing. The increase of GelMA and woodpile design increase the compressive modulus of scaffolds. In vitro evaluation of scaffolds was conducted using dynamic cell seeding for a full coverage of human umbilical vein endothelial cells (HUVECs). Cells on the scaffolds were stable with high density, excellent proliferation, and endothelial phenotype after 7 days of culture, which indicated an excellent biological property of this type of scaffold, as shown in Fig. 34 [196]. Another study also reported using gelatin methacrylamide for facilitating the bioprinting of gelatin based scaffolds [197]. Gelatin methacrylamide hydrogels were mixed with HepG2 cells for 
scaffolds printing using a bioplotter. In vitro characterizations showed a high cell viability of more than $97 \%$ in cell-laden gelatin methacrylamide scaffolds.

Other natural biopolymers, such as starch, cellulose and chitosan have also been used for different AM technologies [198-200]. However, they are mostly used as biocomposites for achieving better mechanical and biological properties. Lam et al. reported a binder jetting method for fabricating porous starch/dextran/gelatin composite scaffolds [198]. Crack free porous scaffolds were prepared with different pore designs. Then scaffolds were infiltrated in PLLA-PCL copolymer solution for improving their mechanical properties. The "rod_censolid" design show the comparable mechanical property to dense cylinder featured with a compressive stiffness of $55.19 \mathrm{MPa}$ and an initial yield strength of $1.77 \mathrm{MPa}$. Salmoria et al. reported a way to make starch-cellulose and cellulose acetate scaffolds using direct SLS [199]. Laser parameters were optimized to avoid material degradation and mechanical failure. The best mechanical property was achieved using 106-125 $\mu \mathrm{m}$ of cellulose acetate. The maximum elastic modulus and ultimate strength were $193.8 \mathrm{MPa}$ and 3.729 MPa, respectively.

A material extrusion method was used for preparing porous chitosan scaffolds to study effects on different surface properties, architecture and controlled pore geometry on cytokine profile of human monocytes/macrophages [201]. Scaffolds with orthogonal and diagonal pore design were prepared with a porosity of $75.6 \%$ and $79.1 \%$, respectively. This method provides an excellent 3-D platform to study cell responses by the cytokine profile. In another study, chitosan was used for making scaffolds by a 3D plotting system [200]. Pure chitosan and chitosan crosslinked with pectin (CP) and genipin (CG) were used as plotting materials. $\mathrm{CP}$ and CG significantly increased the compressive modulus of scaffolds from $130.8 \pm 30.9$ $\mathrm{kPa}$ to $244.3 \pm 36.8 \mathrm{kPa}$ and $256.2 \pm 43.8 \mathrm{kPa}$, respectively. In addition, the in vitro characterizations using osteoblast cells showed their biocompatibility, as shown in Fig. 35 [200]. Yan et al. reported a novel 3D cell-assembly technique for printing hepatocytes and gelatin/chitosan biocomposite together for tissue engineering applications [202]. Hepatocytes remained viable and conducted biological functions on 3D printed constructs. A similar cell-assembly technique was also used for printing gelatin/alginate/fibrinogen biocomposite and adipose-derived stromal (ADS) cells to make an in vitro 3D model of the metabolic syndrome [203]. Results indicated that this 3D model successfully mimicked the metabolic syndrome, which was beneficial to study treatments for metabolic syndrome.

3.3.2. Synthetic biopolymers-Even though natural biopolymers have many advantages, such as excellent biocompatibility and degradability, there are limitations, such as high cost and mediocre physical, chemical, mechanical properties and their immunogenicity. Synthetic biopolymer can be used to make up for those disadvantages. Polycaprolactone (PCL) is a commonly used biopolymer with excellent biocompatibility and biodegradability [204]. The slow degradation of PCL in human body fluid is caused by hydrolysis of ester linkages, which results in harm-free products, e.g., carbon dioxide and water [205]. In addition, because of its excellent processing ability, it has been widely used as a feedstock material for extrusion based AM. Hutmacher et al. reported a FDM method for preparing porous biodegradable PCL scaffolds [206]. Compressive stiffness and compressive yield strength of PCL scaffolds were affected by build patterns, lay-down 
patterns of $0 / 60 / 120^{\circ}$ and $0 / 72 / 144 / 36 / 108^{\circ}$. The scaffolds with a $0 / 60 / 120^{\circ}$ lay-down pattern showed better mechanical properties, a compressive stiffness of $41.9 \pm 3.5 \mathrm{MPa}$ and a $1 \%$ offset yield strength of $3.1 \pm 0.1 \mathrm{MPa}$. In vitro study was also conducted using human periosteal and fibroblasts. Results showed that cells showed high proliferation and differentiation on PCL scaffolds. In another study, FDM fabricated porous PCL scaffolds were used for in vitro co-culture of chondrogenic and osteogenic cells for 50 days [207]. Results indicated good cell proliferation, distribution and extracellular matrix formation on PCL scaffolds. Seyednejad et al. reported the use of PCL derivative, poly hydroxymethylglycolide-co- $\varepsilon$-caprolactone (PHMGC), for preparing bone tissue engineering scaffolds via a 3D plotting system [208]. Because of the modification with hydroxyl groups, PHMGC scaffolds showed higher hydrophilicity and faster degradation than PCL leading to its superior biological properties, such as faster cell adhesion and better tissue-scaffold interaction including a larger extent of vascularization than PCL.

Besides the material extrusion, other AM technologies were also applied for making PCL constructs. PCL has semi-crystalline structure and excellent high-temperature stability, which facilitates thermal treatment related AM processes, such as the powder bed fusion. Williams et al. reported a novel bone tissue engineering scaffold manufactured by SLS [209]. Maximum compressive modulus and compressive yield strength of this kind of scaffolds were $67 \mathrm{MPa}$ and $3.2 \mathrm{MPa}$, which was within the range of cancellous bones. Bone morphogenetic protein-7 (BMP-7) and transduced fibroblasts were incorporated on porous PCL scaffolds for in vivo study using pig condyle model. Results indicated significant enhancement of tissue ingrowth, which showed its great potential for bone tissue engineering applications. Stereolithography was also utilized as an indirect method for making bi-porous PCL scaffolds [210]. Two types of pores, global macropores (300-400 $\mu \mathrm{m})$ and local micropores $(30-100 \mu \mathrm{m}$, and $1-5 \mu \mathrm{m})$, were prepared by negative mold removal and salt leaching, respectively. The inner architecture could be precisely controlled by the design of the negative mold, while porosity and pore size could be controlled by varying the salt amount and diameter.

Another group of polyester based synthetic biopolymers is polyglycolic acid (PGA), polylactic acid (PLA) and their copolymer poly(lactic-co-glycolic acid) (PLGA), which have drawn lots of attention for biomedical applications. PLA can be produced by various renewable resources, such as corn starch, tapioca roots, and sugarcane. In addition, there are several other forms of PLA, such as poly-L-lactide (PLLA), poly-D-lactide (PDLA) and poly(L-lactide- $c o$-D,L-lactide) (PLDLLA). These biopolymers have different biodegradation rates according to their different crystallinity. For example, biodegradation of PLA is faster than PDLA due to the lower crystallinity of PLA. Kanczler et al. reported the use of surface selective laser sintering (SSLS) to prepare porous PLA scaffolds [211]. For SSLS, only a thin surface layer melting $(\sim 200 \mu \mathrm{m})$ happens under laser in order to prevent the degradation caused by the overheating [212]. Different designs of SSLS-PLA scaffolds were successfully prepared. Human fetal femur-derived cells were loaded on the scaffold for in vitro characterization. Then scaffolds were also implanted in a murine femoral segmental defect model for in vivo evaluations. Results showed excellent biocompatibility and biodegradability of these scaffolds in vitro and in vivo, as shown in Fig. 36 [211]. Serra et al. reported a high-resolution material extrusion technology for making PLA based composites 
scaffolds [213]. The addition of PEG into PLA decreased the glass transition temperature of the polymer blend, which improved the 3D-plotting processing resolution without affecting physicochemical properties of the composite scaffolds. The addition of 5\% w/w PEG was able to decrease the glass transition temperature from $59.15 \pm 0.16^{\circ} \mathrm{C}$ to $40 \pm 0.6{ }^{\circ} \mathrm{C}$, which allowed the $\mathrm{AM}$ of the polymer at a lower temperature. Compressive modulus of this type of scaffold was $99.81 \pm 3.55 \mathrm{MPa}$. In addition, biological properties of scaffolds were tested in vitro using rat mesenchymal stem cells. Scaffolds showed excellent cell adhesion on the scaffold surface.

PGA is also a biocompatible and biodegradable polymer. However, since PGA is very sensitive to hydrolytic degradation leading to a fast degradation rate, its biomedical application is limited to suture materials only. The copolymer of PLA and PGA, i.e. PLGA, is commonly used in AM processes. Lee et al. reported an indirect 3D printing process for creating porous PLGA scaffolds [214]. Except the pores designed on the mold, sucrose powder was mixed with PLGA for creating porosity within the matrix of scaffolds. The final scaffold was able to support intestinal epithelial cell (IEC6) growth in culture. PLGA was also processed by multi-head deposition to make porous scaffolds for tissue engineering application [215]. The final scaffolds were featured with a porosity of $47 \%$, a pore size of $200 \mu \mathrm{m}$ and a compressive strength of $1 \mathrm{MPa}$. In addition, cells showed high proliferation level by MTT assay. In a follow-up study, the same group reported a PCL/PLGA composite scaffolds using the same method [216]. The composite scaffold increased the compressive strength to 3.2 MPa. In addition, scaffolds showed a comparable proliferation to PLGA scaffolds in vitro using hBMSCs.

Polypropylene fumarates (PPF) is another promising biocompatible and biodegradable polymeric biomaterial. It degrades to propylene glycol and fumaric acid which are also biocompatible [217]. PPF is normally processed by a vat polymerization method since it has unsaturated sites in its backbone for photo-polymerization. Lee et al. reported effects of laser parameters and resin formulations on the stereography of PPF/DEF scaffolds [218]. DEF was used to dissolve PPF and reduce the viscosity of the solution. The photoinitiator used for the photopolymerization was bisacrylphosphrine oxide (BAPO). Resin composition and laser parameters, such as critical exposure $\left(\mathrm{E}_{\mathrm{c}}\right)$, penetration depth $\left(\mathrm{D}_{\mathrm{p}}\right)$, laser speed and energy, were optimized to fabricate PPF/DEF scaffolds for achieving higher compressive modulus. The reported maximum compressive modulus is $140 \pm 6.1 \mathrm{MPa}$. Porous scaffolds using only PPF were prepared as well for studying effects of pore size on compressive modulus. Decreasing the pore size increased the compressive modulus. In a follow-up study, the same group reported in vitro assessment of PPF/DEF scaffolds using fibroblasts [217]. This method could precisely control pore size, porosity, interconnectivity, and pore distribution. In addition, scaffolds showed cell adhesion. Controlled release of BMP-2 was also reported using similar PPF/DEF scaffolds fabricated by microstereolithography [219]. PLGA microspheres were used to control the release of BMP-2. In vitro results using preosteoblasts indicated improved osteoblast proliferation and differentiation compared to scaffolds prepared by a particulate leaching/gas foaming method. In vivo characterizations in a rat cranial bone defect model showed that BMP was able to significantly enhance bone growth after 11 weeks of implantation, as shown in Fig. 37 [219]. 
Polyetheretherketone (PEEK) is a new emerged synthetic biopolymer for orthopedic applications due to its excellent bio-compatibility, great ability for thermal processing, appropriate strength and stiffness that are close to bones, and availability for radiographic assessment [220-224]. Despite all these advantages, the major disadvantage of PEEK is that it is relatively bioinert, which is not beneficial for new bone growth and osseointegration. The most effect way to improve the bioactivity of implants manufactured by PEEK is to introduce some porosity within the PEEK constructs [225-227]. AM is an excellent tool to create complex design and control pore size, shape as well as porosity. Two major AM methods were used for making porous PEEK tissue engineering scaffolds, SLS and FDM. Tan et al. reported a PEEK-HA composite tissue engineering scaffolds manufactured by SLS [228]. Parameters for SLS, such as scan speed, laser power, and part bed temperature, were optimized for making the composite tissue engineering scaffolds. Further study was conducted by Wilmowsky et al. for making PEEK, PEEK with $2 \mathrm{wt} \%$ carbon black, PEEK with $1 \mathrm{wt} \%$ carbon and $10 \mathrm{wt} \% \beta$-TCP, and PEEK with $1 \mathrm{wt} \%$ carbon and $10 \mathrm{wt} \%$ bioglass scaffolds [229]. Carbon black was added for improving the flowability of PEEK and $\beta$-TCP/ bioglass was added for enhancing the bioactivity of PEEK scaffolds. Results showed that the presence of bioglass significantly increased the osteoblast attachment and proliferation compared to other compositions. For FDM, Vaezi et al. reported porous PEEK parts manufactured by syringe and filament based FDM systems. An ambient temperature of $80{ }^{\circ} \mathrm{C}$, a build plate temperature of $130^{\circ} \mathrm{C}$, a nozzle temperature between $400{ }^{\circ} \mathrm{C}$ and $430{ }^{\circ} \mathrm{C}$, and an extrusion rate of $2.2 \mathrm{mg} / \mathrm{s}$ were the optimized parameters of FDM for avoiding the warpage and delamination of final PEEK constructs. Results showed a compressive yield strength of $102.38 \mathrm{MPa}$ and $29.34 \mathrm{MPa}$ and a compressive yield strain of 0.056 and 0.044 for scaffolds with $0 \%$ and $38 \%$ porosity, respectively. It presented the great potential for this type of scaffolds to be used in load-bearing applications.

Calcium polyphosphate (CPP) is another interesting synthetic biopolymer with inorganic composition [230]. The composition of CPP can be written as $\left[\mathrm{Ca}\left(\mathrm{PO}_{3}\right)_{2}\right]_{n}$, which is very similar to calcium phosphate material, which leads its excellent bioresorption, biocompatibility, and osteoconductivity. In addition, the degradation of CPP is a hydrolytic reaction between $\mathrm{P}$ and $\mathrm{O}$ within different chain segments resulting in orthophosphates followed by further degradation to naturally metabolized products [231,232]. CPP has been widely used to fabricate porous scaffolds using AM processes for biomedical applications. Shanjani et al. reported porous CPP scaffolds using binder jetting method [233-235]. The printing direction has significant effects on the compressive strength. Paralleled printing and compression directions could increase the compressive strength compared to perpendicular directions. The maximum reported compressive strength was as high as $50.17 \pm 4.74 \mathrm{MPa}$ with a mean pore size of $56 \mu \mathrm{m}$, a pore size range between 20 and $150 \mu \mathrm{m}$, and a volume porosity of 35\% [234]. After 6 weeks of implantation in rabbit femur, between 30 and $40 \%$ porosity was filled with new bone along with 7-9\% CPP weight loss due to biodegradation [235].

3.3.3. Polymer-ceramic composites-Both polymer and ceramic materials are widely used as biomaterials because of their own advantages as discussed before. However, ceramics are inherently brittle while polymeric materials are flexible. Hence the potential of 
combining these two types of materials is very promising. Various AM processes have been used to manufacture ceramic-polymer composites. In SLS or SLM, when combining these two different materials together, polymer content usually dominates in the composite, which melts under laser powder and acts as a glue to bind ceramic particles. Since melting point and decomposition temperature for some biodegradable polymers are close, the temperature control during AM processing is very important. In the vat polymerization, polymer dominates the composite as well since the only polymer can have photopolymerization. In the material extrusion or 3D plotting, polymer still dominates the composite because of its lower melting temperature or better flowability. However, the amount of ceramic can significantly influence AM processing parameters such as flowability of powders in binder jetting and viscosity of suspension in vat polymerization.

The most common technology for producing polymer/ceramic composite architecture is material extrusion, such as FDM and 3D plotting. For the material extrusion of polymer/ ceramic composite, the key factor is to prepare printable composite filament. PP/TCP composite was used to fabricate porous scaffolds using FDM for bone tissue engineering applications [236]. Different pore structures were successfully prepared by FDM with excellent dimensional accuracy showing its great processing ability. Scaffolds with $160 \mu \mathrm{m}$ pore size and $36 \%, 40 \%$, and $52 \%$ volume fraction porosity were prepared for mechanical property characterizations. A maximum compressive strength of $12.7 \mathrm{MPa}$ and a maximum modulus of $264 \mathrm{MPa}$ were reported for scaffolds with $36 \%$ porosity. In addition, scaffolds showed good osteoprecusor cells adhesion and proliferation in vitro, as shown in Fig. 38 [236]. Korpela et al. reported a porous PCL/bioglass composite scaffolds prepared by FDM process [237]. $10 \mathrm{wt} \%$ of bioglass was added to PCL for preparing FDM filaments. Bioglass particles were successfully embedded into porous PCL matrix scaffolds. The compressive modulus enhanced from $104 \mathrm{MPa}$ to $147 \mathrm{MPa}$ when adding bioglass. Scaffolds also showed excellent cell adhesion and proliferation in vitro using fibroblasts.

Compared to FDM, 3D plotting builds structures by direct deposition of viscous material from a fine nozzle. The viscous material is extruded under pressure using compressed air. Therefore, it is a heat-free AM process, which is beneficial to incorporate live cells or tissues in the scaffold during fabrication [238,239]. 3D-plotting was used to fabricated PLA/PEG/ bio-glass composite scaffolds with high resolution [213]. Bioglass particles were evenly distributed within the polymer matrix, which enhanced the hydrophilicity of the scaffold surface, mechanical properties as well as the bioactivity. In addition, scaffolds with the bioglass showed well-spread cell morphology on the surface indicating its excellent bioactivity. Luo et al. reported a novel hierarchical mesoporous bioglass/alginate composite scaffolds manufactured by 3D-plotting system [239]. Two patterns, XY and XXYY, were designed for 3D-plotting followed by crosslinking using $\mathrm{CaCl}_{2}$ solution and drying. Mechanical, biological and drug release study were performed with those scaffolds, as shown in Fig. 39 [239]. Results showed the XXYY design significantly improved the pore interconnectivity and dimensional accuracy of scaffolds. The addition of $50 \%$ mesoporous bioglass increased the compressive strength from 2.1 MPa to 6.2 MPa for composite scaffolds using XY design. In addition, the incorporation of mesoporous bioglass controlled the release of dexamethasone and improved the bioactivity of scaffolds. 
Wiria et al. reported a PCL/HA composite scaffold by SLS technique for tissue engineering applications [240]. PCL is an excellent material for SLS because of its high thermal stability. HA has high compatibility and bioactivity. The combination of those two materials is very promising in tissue engineering. Laser power, scan speed, and HA weight percentages were optimized for achieving excellent mechanical properties. A yield stress at $2 \%$ strain offset of $11.54 \pm 0.80 \mathrm{MPa}$ and a Young's modulus of $102.06 \pm 11.26 \mathrm{MPa}$ were achieved using 10 wt $\%$ HA and $90 \mathrm{wt} \%$ PCL. Bioactivity test using SBF immersion and in vitro characterizations using Saos-2 cells showed good biocompatibility. Duan et al. also reported a SLS method using calcium phosphate (Ca-P)/poly(hydroxybutyrate-co-hydroxyvalerate) (PHBV) and carbonated hydroxyapatite (CHAp)/poly(L-lactic acid) (PLLA) to manufacture scaffolds with interconnected porosity [241]. The maximum compressive strength was less than $1 \mathrm{MPa}$, but showed good cell adhesion, proliferation and differentiation during in vitro characterizations using SaOS-2 cells. SLM technology was also utilized for preparing composite scaffolds using TCP/PDLLA composite for bone implant applications [242]. The addition of TCP could reduce the acid environment caused by the degradation of PDLLA. A maximum four-point bending fracture strength of $23 \pm 1 \mathrm{MPa}$ using $0.5 \mathrm{~W}$ laser power and $50 \%$ PDLLA/50\% TCP was achieved for TCP/PDLLA composite scaffolds.

Vat polymerization method can also be used by formulating homogeneous composite suspension followed by photopolymerization to encapsulate ceramic particles within the polymer matrix. Ronca et al. reported a novel SLA method to process poly(D,L-lactide)/ nanosized hydroxyapatite (PDLLA/nano-HA) composite scaffolds [243]. Methacrylate groups from PDLLA can be crosslinked to rigid polymer network, but the high crosslinking density of PDLLA makes the structure quite brittle. The addition of HA can improve its mechanical and biological properties. 0, 5, 10, and $20 \mathrm{wt} \%$ were used for the optimization of the vat polymerization. $5 \mathrm{wt} \%$ of nano-HA was finally used for fabricating scaffolds with the Schwarz pore network because of its lower viscosity and better processing ability. The flexural modulus increased from $3.1 \pm 0.4 \mathrm{GPa}$ to $4.1 \pm 0.3 \mathrm{GPa}$ in the presence of $5 \mathrm{wt} \%$ of nano-HA. The part was precisely in agreement with the design file and the ceramic particles were well dispersed within the pore surface. In another study, bioglass was combined with photocrosslinkable PCL for composite scaffolds fabrication [244]. The modified PCL, methacrylated poly( $\varepsilon$-caprolactone), was mixed with bioactive glass ( $\mathrm{S} 53 \mathrm{P} 4)$ for creating porous composite scaffolds. The compressive modulus was measured for scaffolds with different amount of bioglass. With the increase of bioglass content, the compressive modulus of scaffolds increased, and the maximum value reported in this study was $3.4 \mathrm{MPa}$ using 20 wt\% bioglass. Bioglass was evenly distributed on the surface of the PCL matrix, which enhanced the bioactivity of the scaffolds as measured via precipitation of apatite in SBF, and improved fibroblasts adhesion and proliferation in vitro.

Another AM method, binder jetting, is also available for manufacturing polymer ceramic composites. Inzana et al. reported a binder jetting method using collagen as the binder to make composite scaffolds with calcium phosphate powder in the powder bed [245]. Collagen was incorporated with phosphoric acid as the binder solution. $8.75 \mathrm{wt} \%$ of phosphoric acid was used with a collagen concentration up to $2 \mathrm{wt} \%$. The addition of collagen significantly improved flexural strength, toughness, in vitro cell proliferation as 
well as in vivo bone ingrowth in murine femoral defect model after 9 weeks. Table 8 summarizes different AM approaches towards ceramic-polymer composites.

\section{Applications of AM in biomaterials and biomedical devices}

There are two major sets of applications for AM in biomaterials. Orthopedic and dental implants are the first kind of applications in which AM techniques are used to manufacture devices that are patient-matched or difficult to manufacture otherwise. Most of those devices are metal based, however, other materials have also been used. Another area of application is tissue engineering. Generally, cells need certain three-dimensional support with pores to attach, grow and differentiate into functional tissues or organs [256-258]. AM techniques are especially outstanding in making architectures with both micro-and macroporosity. Traditional top-down AM system can assemble cells and tissue growth factors onto a temporary structure for further proliferation and maturation to fully functional tissues. This method has achieved scientific and clinical success in the past twenty-five years, but it also has significant limitations, such as the manufacture of a functional and controlled histoarchitecture, and the control of mass transfer including nutrients and wastes. Recently, a new bottom-up system, also called bioprinting, has been used for tissue engineering applications. This technique can incorporate features of individual characteristics towards guiding the tissue maturation via enhancement of cellular self-sorting and self-assembly capabilities $[259,260]$. There are three major bioprinting systems available - inkjet bioprinter, microextrusion bioprinter, and laser-assisted bioprinter, as shown in Fig. 40 [261,262]. Inkjet bioprinters can utilize heating generated air-pressure pulse or piezoelectric pressure for droplet deposition. Pneumatic or mechanical (piston or screw) dispensing systems are used for microextrusion bioprinter to extrude continuous materials. And laserassisted bioprinter applies laser energy on energy-absorbing layers to generate force for propelling cell-containing material to the substrate [262].

In this application of AM in biomaterials section, metal based implants or devices, and tissue engineering structures are discussed in detail. Tissue engineering is further divided into hard tissue, soft tissue, and organ tissue engineering. Both top-down and bottom-up AM systems are introduced for their applications in tissue engineering, as shown in Table 9.

\subsection{Metallic implants to improve osseointegration}

Metallic implants are commonly used in the human body to replace load-bearing joints namely the hip and the knee joints. These implants are called total hip replacement (THR) and total knee replacement (TKR). The basic design concepts for these implants remained the same for the past few decades. These load-bearing implants demand properties such as high compressive strength, and resistance to wear and bio-corrosion. In the configuration involving metal-on-metal contact, concerns related to metal ion release are very high. However, for metal-on-polymer devices, concerns related to osteolysis is also quite high due accumulation of polymeric wear debris in certain locations. Therefore, in any design configuration, wear induced damage cannot be eliminated; attempts can only be made to minimize the impact. Moreover, dense metallic implants in the body with high stiffness can cause stress shielding or weakening of the neighbouring bones, a problem arising out of the 
differences in stiffness between the surrounding bone and the implant. In recent years, application of new AM strategies has attempted to diminish these long-standing problems.

Cobalt chromium and titanium based alloys are primarily used in THR and TKR applications. Components for THR and TKR such as hip stem or the ball and socket joints are manufactured using conventional methods like casting and forging. Using additive manufacturing methods such as EBM, EBDM, SLS/SLM and LENS ${ }^{\text {TM }}$ and 3D Inkjet printing, different load-bearing and patient specific implants have been manufactured. As discussed earlier, these can be either made from metallic powders or from wire feed. Parts made from such AM methods generally exceed the strength requirements due to the rapid solidification rates in AM that yield a fine-grained microstructure and thus higher strength. Instead of fully dense implants via casting or forging, AM can manufacture implants with designed porosity. Porosity in the implants can lower the effective stiffness and reduce stress shielding related concerns [61]. Secondly, surface porosity can offer anchoring sites for the surrounding tissues during healing and offer enhanced early stage osseointegration. Both of these implications would lead to a more gradual transfer of stresses across the human bone/ tissue to the metallic implant, which can increase the life of an implant in vivo. Balla et al. demonstrated LENS ${ }^{\mathrm{TM}}$ processing of porous titanium structures with up to $70 \%$ volume porosity [61,141]. EBM process was also utilized to make similar porous metallic structures [142]. Shishkovsky et al. showed those porous structures indeed improve osseointegration with the bone tissues [151]. Similar dense and porous structures were also fabricated via EBM using CoCrMo alloys [181]. In addition to the structures made from titanium or cobalt based metallic materials, Chou et al. used the inkjet 3D printing technique for the fabrication of porous Fe-30\% Mn alloy structures [298]. These structures showed 36.3\% open porosity and had mechanical strength similar to that of the natural bone. These porous structures also showed good in vitro cytocompatibility after cell culture using MC3T3-E1 pre-osteoblast cells.

Apart from introducing porosity using AM to enhance osseointegration and reduce stress shielding, there are many articulating surfaces in between the implant components that undergo relative motion and hence wear. These areas have to strictly avoid porosity since porosity will elevate the wear rate and increase the release of metallic ions in vivo. Another possible issue is the inadequacy of the compressive strength of the porous structures. To counter these two issues, load-bearing components can be fabricated with structural porosity gradation. These components would essentially have select areas with a high porosity which can promote osseointegration and promote gradual stress transfer. For example, those areas can be the outsides of an acetabular cup component that comes in direct contact with bone. There can also be areas that should be dense structure such as inside the cup to provide the mechanical strength and wear resistance to the implant. We can thus envision an implant that has porous outside layers of a certain depth and a denser inner core. Such a structurally graded implant would be a challenge to fabricate via most conventional techniques but can be easily realized by additive manufacturing. The realization of such a component with a structural gradation of porosity with denser core and porous exterior was done via EBM powder bed additive manufacturing of Ti6Al4V powder [143,299]. 
To counter the problem of poor osseointegration between the human tissues and the metallic implant, researchers also proposed surface modification or coating. The most common coating investigated for this purpose was a coating of calcium phosphate ceramic. As discussed in the materials sections, direct energy based additive manufacturing techniques such as laser melt injection and LENS ${ }^{\mathrm{TM}}$ have been effective in creating a calcium phosphate coating on metallic implants. This can either be done as a plain coating on the metallic material or as a compositionally graded coating. Many techniques such as plasma sprays and thin film techniques are also capable of processing similar quality of coatings. However, additive manufacturing methods can give a new dimension to this old concept of bioceramic coating. Using tools like LENS $^{\mathrm{TM}}$, it is possible to have coatings on complex shapes and geometries. Most traditional coating processes are a line of sight techniques, meaning it is a challenge to process coatings on contours and curved surfaces. This problem can be solved using additive manufacturing. Furthermore, not just a bioceramic coating but a porous bioceramic coating can also be processed on metallic implants. This will not only increase the biocompatibility of the device but will also lead to a better osseointegration than a dense implant, or porous implant or a dense bioceramic coating. Additive manufacturing can be exploited for this goal in two ways. First, the metallic devices can be manufactured using any traditional methods and bioceramic coatings can be applied via additive manufacturing route. In the second approach, processing of the metallic implant as well as the bioceramic coating can be done simultaneously via additive manufacturing route. The deciding factors for this would be the complexity of the design of the implant. Apart from the processing of dense and porous bioceramic coatings, additive manufacturing can also be used to process novel metal-ceramic composites as new materials for load bearing applications. This will be discussed in the following section.

\subsection{Devices with enhanced wear resistance}

Solving the problems of wear, biocorrosion, and metal ion release is the other prime area where AM can help to innovate next generation of biomedical devices. The first and most fundamental approach to reducing wear in between any contact surfaces is by using a hard surface. Since the wear rate is inversely proportional to the hardness of the surfaces in contact, the easiest way to improve the tribological properties is to improve the surface hardness. Using AM, the hardness of the contacting surfaces can be improved in several ways. Different metal/ceramic composites have been demonstrated by laser melt injection since 1989. Reinforcing of Ti based metallic materials with silicon carbide [58], tungsten carbide [59], titanium boride-titanium nitride [160,161] and titanium oxide [63] were successfully demonstrated to increase the surface hardness and wear resistance of the base material. Apart from these, harder metallic materials were also used to improve the tribological properties of Ti based materials such as Tantalum [173,174]. These coatings not only had better tribological properties but also showed good cell proliferation during in vitro cell culture. Similarly, niobium was also investigated [177]. Cobalt based alloys were also investigated for surface modification using silicon carbide and laser cladding process [57]. Other ceramic systems such as tungsten carbide, titanium carbide have also been studied and found to be successful in alleviating wear damage in the alloys. 
In another approach, ceramic coatings can also be used on metallic implants. Ceramics such as alumina, zirconia, silicon carbide and more commonly titanium nitride have been demonstrated to reduce wear in metallic materials. However, AM of ceramics can be a challenge at times. Hence, compositionally graded metal-ceramic coatings could be used. Such ceramic or metal-ceramic composite coatings processed via AM have several advantages. First, the ability to coat or process complex shapes and surfaces, which is not easy in conventional manufacturing or coating processes. The second advantage is the formation of a strong interface with the substrate material. AM methods such as laser melt injection and LENS ${ }^{\mathrm{TM}}$ are extremely focused and do not create excessive heat affected zones. By avoiding such heat affected zone, these processes create a strong interface since they still adequately melt the substrate. However, as a hard ceramic coating with properties different than that of the substrate is created, due to low heat affected zone and controlled dilution in the substrate, the inherent properties of the substrate are preserved. Via AM, it is thus possible to achieve controlled surface modification in the existing metallic implants.

Another approach of reducing wear is using a solid lubricant. For biomedical applications, the most common additive would be calcium phosphate. The addition of calcium phosphate to CoCrMo alloy and processing it using LENS ${ }^{\mathrm{TM}}$ based additive manufacturing has been shown to reduce wear in the alloy [300]. Another advantage towards current load-bearing implant is the ability of AM to achieve selected area modification. Essentially, it may not be economical or time-consuming to coat an entire surface of an implant. In cases like these, finite element modeling can help to determine the areas in an implant that undergoes the highest wear and corrosion damage. In certain cases, explanted implants are also studied to understand the areas of preferential wear as well as the mechanisms of wear and bio-tribocorrosion. Such areas can then be selectively modified to minimize wear induced damage. One approach is to use computational models to predict and selectively modify or enhance the areas that may undergo wear related damage [301]. An example is shown in Fig. 41a and $\mathrm{b}$ of a computational model to predict wears after 50 million cycles. Careful observation of load bearing implants that have undergone tribological damage can also yield very valuable knowledge about the wear areas and wear mechanism of the implants such as shown in Fig. $41 \mathrm{c}$ and $\mathrm{d}$ [302]. These findings can then be used for enhancing the mechanical performance of the next generation of implant.

Such areas when identified can be selectively modified either by metal based intermetallic coatings or by a metal-ceramic hard facing coating or by a metal ceramic that acts as a solid lubricant during wear. These could be processed in situ using additive manufacturing techniques such as laser melt injection or LENS ${ }^{\mathrm{TM}}$. Implants can also be processed with these areas in mind from the bottom up approach using methods such as EBM or SLS/SLM.

\subsection{Hard tissue engineering}

Hard tissue is also called mineralized tissue which combines minerals with the soft organic matrix that provides structural support for other tissues. Typical hard tissues are bones, teeth, and cartilage. They play important roles in our body. However, because of aging, accidents or certain congenital diseases, they need replacements and repair. In this subsection, bone, tooth and cartilage tissues by top-down or bottom-up AM approach are discussed. 
4.3.1. Bone-The top-down AM process was first used with HA powders. Fierz et al. reported the use of inkjet 3D printing to make anisotropic HA scaffolds with a repeating two layers design for bone tissue engineering [263]. The design of millimeter scale center channels and perpendicular micro-pores was similar to cancellous and cortical bones. The tomographic characterization showed the interconnected structure of scaffolds in a nonedestructive manner. Then osteogenic-stimulated progenitor cells were loaded to test biological properties of those scaffolds. The osteoblast-like cell morphology near the dissolved HA indicated that it was promising for clinical use. Besides HA, TCP is also widely used in bone tissue engineering scaffolds because of its better biodegradability than HA. Hip Stem, human pelvis and Washington State University (WSU) logo were successfully built by TCP using 3D printing as shown in Fig. 42 [94]. Fielding et al. reported a novel 3D printed porous TCP composite scaffolds for in vivo characterization in rat femurs [264]. Pure TCP and $\mathrm{SiO}_{2} / \mathrm{ZnO}$ TCP scaffolds were prepared by binder jetting 3D printer with around $300 \mu \mathrm{m}$ of pore size after sintering. Scaffolds were inserted into rat femurs for 6, 8, 12 and 16 weeks and taken out for histomorphology and immunohistochemistry analysis. Results showed that the presence of $\mathrm{SiO}_{2}$ and $\mathrm{ZnO}$ induced the new bone formation, collagen production, osteocalcin expression and vascularization, as shown in Fig. 43 [264].

Besides the inorganic composition, the organic content is around $20 \%$ of the natural bone. The composition of the organic part is mainly collagen, which is an elastic protein that improves the fracture resistance of the bone. Hence, the preparation of collagen-calcium phosphate composites architecture has drawn lots of attention. Inzana et al. reported an AM method for making collagen-calcium phosphate composite scaffolds using collagen containing binder for printing [245]. Collagen was incorporated with calcium phosphate matrix by mixing with the phosphoric acid binder solution. Other polymer-ceramic composites were also used for making bone tissue engineering scaffolds through FDM. Kim et al. reported PLGA/ $\beta$-TCP/HA nanocomposite scaffolds for in vivo studies in a rabbit femoral defect model [303]. Scaffolds were prepared using PLGA/ $\beta$-TCP nanocomposites using FDM followed by coating using HA. They were implanted into a rabbit femoral defect model for 6 and 16 weeks showing their great biocompatibility and potential for drug delivery applications.

For the bottom-up system, cells can be encapsulated in hydrogels as bio-ink for printing, which is also called cell-laden hydrogel method. Fedorovich et al. reported different hydrogels with bone marrow stromal cells (BMSCs) laden for the bio-printing of bone tissue engineering scaffolds [238]. Cell viability and differentiation were found not affected by the extrusion process of the bioprinting when compared to unprinted cells. In addition, it was demonstrated that this method provided a way to print cells in different populations which had the potential to combine BMSCs and endothelial progenitors for vascularized bone grafts. In addition, bioprinting can also be utilized to stack up different materials or cell patterns to construct a functional graft. Ker et al. created a novel bioprinting method via a two steps process [266]. The first step was to make a highly oriented sub-micron fibers by Spinneret-based Tunable Engineered Parameters (STEP) technique. The next step was to print fibroblast growth factor-2 (FGF-2) or BMP-2 for controlling cell differentiation. In 
vitro results indicated that cell alignment followed the fiber length direction. In addition, the FGF-2 and BMP-2 patterns were able to promote differentiation of tenocyte and osteoblast, respectively. Unprinted STEP fibers showed the differentiation to myocytes, as shown in Fig. 44 [266].

4.3.2. Tooth-Tooth is another major mineralized tissue in our body, which is constituted of four major parts, enamel, dentine, cementum, and pulp. Enamel and dentin are highly mineralized tissues with $96 \mathrm{wt} \%$ and $70 \mathrm{wt} \%$ mineral content, respectively, which provide excellent mechanical properties for tooth [304]. No cellular substances are present in enamel and dentin. Cementum is a less mineralized tissue with around $45 \%$ inorganic substance and cellular bodies, such as cementoblasts, surrounds the root of the tooth. Pulp has little mineral content but lots of living connective tissues, which contains blood vessels, nerves and cellular bodies, such as odontoblasts, fibroblasts, preodontoblasts, macrophages and $\mathrm{T}$ lymphocytes. These four parts construct a very delicate system for proper functions of our teeth. The mineral content in the tooth is mainly hydroxyapatite, which is similar to the bone tissue. In addition, the tooth is a highly porous tissue in microscale.

Traditional manufacturing methods such as lost-wax and plaster casting, have their limitations including difficulties for making complex internal structures and low costeffectiveness. However, AM is a promising solution for dental prosthesis fabrication since it is advantageous in making complex designs with high accuracy in a shorter time. Kasparova et al. reported a study to evaluate the manufacturing accuracy of two 3D printing systems, open source system RepRap and commercially available 3D printer [305]. The plaster model was used as a standard to create a digital 3D image for 3D printing. Linear measurements of selected points on the dental arches of upper and lower jaws were conducted to analyze the accuracy of 3D printed parts. The statistical evaluation showed that 3D printer had better dimensional accuracy than traditional plaster casting for making dental implants. In another study, vat polymerization methods, digital light processing and jetted photopolymer and binder jetting were used for fabricating denture models [306]. The mean systematic differences calculated from linear measurement between AM models and the plaster model were very little (between $0.02 \mathrm{~mm}$ and $0.25 \mathrm{~mm}$ ) indicating the high accuracy and reproducibility of AM produced denture models.

Different AM methods are used to fulfill requirements of certain dental implants. Kim et al. published a novel direct metal laser sintering (DMLS) method to make three-unit fixed dental prostheses for comparison to the tradition lost-wax casting [268]. Cobalt-chromium alloy was used for DLMS and nickel-chromium alloy was the starting material for lost-wax casting. Three important parameters, internal gap (IG), marginal gap (MG) and marginal discrepancy (AMD), were measured on three-unit fixed dental prostheses via both DLMS and lost-wax casting. Even though the marginal fit of DLMS processed implants was a little inferior to the one of lost-wax cast parts, it was still promising for clinical use. Synthetic onlay grafts are frequently used before dental implants insertion to increase the alveolar bone volume for improving osseointegration and stability of the implant. However, the vertical bone augmentation of synthetic onlay grafts is still inferior compared with autologous onlay grafts. Tamimi et al. reported a 3D printing method to fabricate dicalcium phosphate anhydrous (monetite) onlay grafts and its effects on Ti dental implant in vivo 
[269]. Binder jetting method was used to create synthetic onlay grafts using $\beta$-TCP powder as matrix and $20 \%$ of diluted phosphoric acid $\left(\mathrm{H}_{3} \mathrm{PO}_{4}\right)$ as binder. The onlay grafts were first inserted into the calvarial bone of female rats and then secured with osseosynthesis titanium screws. After healing for 4 weeks, another surgery was conducted to remove screws and insert titanium dental implant above onlay grafts. The onlay design with macroporosity enhanced both bone volume and bone height compared to the design with microporosity only. Nearly half of the monetite from the onlay was degraded after 8 weeks of implantation. In addition, the titanium dental implant showed excellent osteointegration with the new bone formation on onlay grafts.

4.3.3. Cartilage-Cartilage is a connective tissue made of chondrocytes and extracellular matrix, such as collagen and proteoglycan, which are primarily located in joints between bones. Cartilage repairs slowly following major damage in an environment with an insufficient blood supply. However, there are surgical procedures, such as microfracture surgery, aimed at providing a helpful blood supply to healing cartilage. The mechanical property of cartilage is in between muscles and bones, which is neither as flexible as muscles nor as rigid as bones. The area that is close to the bone is more rigid than the area that is far from the bone. Near the interface between bones and cartilage, there is also a transitional region from osteoblast to chondrocytes. Using traditional manufacturing methods, it is hard to produce tissues with gradient compositions and mechanical properties. However, AM methods have advantages of controlling the composition of materials and cells in each layer, which is beneficial for cartilage tissue engineering applications.

In the early stage of AM for cartilage tissues, the top-down system was widely explored. Since cartilage is a more flexible tissue than the bone, cartilage tissue engineering scaffolds are usually manufactured using polymer or polymer composites. Sherwood et al. reported a composite scaffold using binder jetting method with gradient material composition and porosity [271]. The design of the scaffold could be separated into three sections. The upper region of the scaffold was fabricated using the PLGA/PLA composite with $90 \%$ macroporosity for improving the cell seeding. The lower region was made by the PLGA/TCP composite with 55\% porosity to enhance its osteogenesis and mechanical properties. The middle region was made by transitional composition and porosity between the upper and lower sections for preventing delamination. The composition combination with the best mechanical property was 35\% NaCl, $15 \%$ TCP and 50\% L-PLGA showing a tensile strength of $5.5 \pm 0.8 \mathrm{MPa}$, an elastic modulus of $233 \pm 27 \mathrm{MPa}$, a yield strength of $13.7 \pm 0.8 \mathrm{MPa}$ and an elastic modulus of $450 \pm 79 \mathrm{MPa}$. It was in line with the magnitude of cancellous bones. In addition, in vitro results indicated that chondrocytes adhered well on this scaffold surface and showed new cartilage formation after 6 weeks, as shown in Fig. 45 [271]. In another study, $100 \%$ interconnected porous scaffolds were fabricated using material extrusion AM technique with poly(ethylene gly col)-terephthalate-poly(butylene terephthalate) (PEGT/PBT) block copolymer [272]. PEGT was hydrophilic and PBT was hydrophobic. Through varying the length and amount of each polymer, copolymers with different properties, e.g., mechanical property and biodegradability, could be achieved. Porosity could also be manipulated by changing the spacing, geometry, and distance. After the optimization, the equilibrium modulus and dynamic stiffness of scaffolds were ranged 
between $0.05-2.5 \mathrm{MPa}$ and $0.16-4.33 \mathrm{MPa}$, respectively, which were comparable to natural articular cartilages. In addition, in vitro dynamic cell culture using chondrocytes and in vivo characterization using nude mice demonstrated that these scaffolds supported chondrocytes attachment and cartilage tissue formation.

Bioprinting can control compositions, cell types, and populations in different areas. Fedorovich et al. reported cell-laden composite scaffolds manufactured using a bioprinting system using biphasic calcium phosphate particles and alginate hydrogel [274]. Different scaffold designs were made to control the porosity of scaffolds. Human MSC and chondrocytes were printed in different areas of the scaffold to stimulate natural cell distribution in the cartilage. Cell viability during the printing was high, and different cells were embedded within their designed compartment without migration. The distinctive tissue formation in vitro and in vivo indicated that such scaffolds were very promising in the cartilage tissue repair. Park et al. created a novel bioprinting system using osteoblasts on Col-1 hydrogels and chondrocytes on hyaluronic hydrogels for the cartilage tissue repair [277]. Those cells which were laden in their native extracellular matrix showed better proliferation and maturation. In addition, a 3D cartilage structure combining these two hydrogels was printed and tested in vitro. After 14 days of cell culture, those two types of cells had high viability and showed healthy cellular functions indicating promising potentials for osteochondral tissue repairs.

In order to improve cell proliferation and differentiation rates, different growth factors can also be combined within the 3D constructs via bioprinting. BMP-2 and transforming growth factor $\beta 1$ (TGF- $\beta 1$ ) were combined with human MSC for improving its proliferation and differentiation [275]. 3D structures with a biochemical gradient of MSC and different growth factors encapsulation were printed to simulate natural fibrocartilage organization. The addition of growth factors enhanced chondrogenesis and osteogenesis through the improvement of related gene expressions. This study showed a promising approach towards fibrocartilage repair. It was also a useful tool to build different models for pharmaceutical testing which used cells directly from patients.

\subsection{Soft tissue engineering}

The main function of soft tissues is to connect and support the surrounding structures and organs in our body. They are mainly composed of elastin, collagen and ground substance, which can absorb the energy under the load to protect other tissues. Some of the important soft tissues include skin, muscle, tendon, ligament, nerve and blood vessel. They have different responsibilities in our body, but the most common cell that is responsible for their functionalization is fibroblast. In order to synthesize artificial soft tissues, AM methods are excellent tools to make polymer or polymer composite constructs since the mechanical property of the polymer is closer to soft tissues than other materials.

4.4.1. Ligaments and tendons-Ligament and tendon are tough fibrous connective tissues, which are made of collagen. The difference between ligament and tendon is parts they are connecting. A ligament connects different bones or teeth, while tendon connects muscles for the movement of bones. Nowadays, tendon and ligament injuries happen quite 
frequently, which almost occupies half of all musculoskeletal injuries [307]. Treatment options for tendon and ligament injuries are through replacement or fixation using grafts and prostheses. Hence the manufacturing of artificial grafts is critical for the success of treating tendon and ligament injuries.

Park et al. reported a negative AM mold casting method for building tooth-ligament complex [280]. The entire complex model was separated into two individual parts, periodontal ligament (PDL) and bone compartments. Wax 3D-printing system was also used for mold fabrication of these two separate parts. Then PGA was used for PDL and PCL was used for the bone compartment for the final fabrication. Manufactured compartments with one human tooth dentin slice on the top were assembled together with BMP-7 added primary human gingival fibroblast (hGF) cells in the bone region and human PDL cells at the PDL interface for in vivo characterizations on two different surgical pockets on the dorsa of immunodeficient 6 week-old NIH III nude mice. In vivo results showed an improved growth of newly formed tooth cementum-like tissue, ligament and bone structures within the toothligament complex, as shown in Fig. 46 [280]. In a follow-up study, a tooth-ligament complex was designed based on the micro-CT image of the periodontal fenestration defect on the cadaver mandible from the an in vivo model [281]. A fiber-guiding channel design was also added for the formation oriented Sharpey's fiber in order to improve the integration of the entire complex. Compared to the random porous design, this fiber-guiding structure enhanced the triphasic tissue regeneration within the tooth-ligament complex in vivo, which is promising towards clinical applications. Besides tooth ligament unit, custom designed implants manufactured by $3 \mathrm{D}$ printing were reported to be applied for treating cranial cruciate ligament deficient stifle joints in dogs [25]. An artificial cage was prepared with the composition of brushite, monetite, and tricalcium phosphate. The structure of the cage was highly permeable with an overall porosity of $59.2 \%$ including a microporosity of $40 \%$ and a designed interconnected macroporosity with pore sizes of $845 \mu \mathrm{m}$. It had a compressive strength of $7.65 \pm 2.01 \mathrm{MPa}$. 3D printed implants showed improvements in biocompatibility and osteoconductivity compared to the standard titanium implant. In addition, complete restoration of dog's limb function was also observed without dog's lameness or any side effects.

Muscle-tendon unit was also manufactured via bioprinting process using polyurethane (PU)/ $\mathrm{C} 2 \mathrm{C} 12$ myoblasts on muscle region and PCL/NIH/3T3 fibroblasts on the tendon region [282]. The final construct had an elastic modulus of $0.39 \pm 0.05 \mathrm{MPa}$ on the muscle side and Young's modulus of $46.67 \pm 2.67 \mathrm{MPa}$ on the tendon side. Bioprinting technique successfully maintained cell viability during printing. Cells in different regions also showed excellent tissue development and differentiation after 7 days' culturing. Results showed great potential for this bioprinting approach to fabricate complex construct with heterogeneous materials and cells.

4.4.2. Blood vessels-Blood vessels are one important part of the circulatory system which is responsible for delivering blood to different parts of the body. There are three major types of blood vessels, namely arteries, capillaries, and veins. Based on their functions, the structure and mechanical properties of different types of blood vessels are very different. Arteries are usually composed of elastic materials with thick wall structure in order to 
withstand the high pressure during the diastole. Capillaries, which are responsible for the nutrient and waste exchange between blood and tissues, are made of an inflexible material with flat and very thin wall structure for easy locating all over the body. The structure and mechanical properties of veins are close to arteries, but the wall thickness of vein is much thinner than arteries since the function of veins, which is transferring blood from capillaries back to the heart, is not under high pressure as arteries. However, the major cell type for the functionalization of different blood vessels is an endothelial cell. Blood vessels are important channels for nutrient and waste circulation. It is also the key point for the final tissue self-functionalization. With the development of AM technology, vascular grafts processed by AM methods have drawn more and more attentions from the scientific community.

Cui et al. reported a novel thermal inkjet printing method for microvasculature preparation using human microvascular endothelial cells (HMVEC) and fibrin [284]. Microchannels were first prepared with a thermal inkjet printer using fibrin. The bio-ink containing HMVEC was printed around the microchannels for the blood vessel formation. In vitro results showed that the printed cells were aligned around the microchannels and formed a confluent cell lining after 21 days, which indicated an effective strategy for microvasculature fabrication. Bioprinting technique was also applied for vasculature grafts fabrication. Skardal et al. published a study for the fabrication of vessel-like constructs using murine fibroblasts (NIH 3T3) encapsulated by tetra-acrylate derivatives (TetraPAcs)/thiolated hyaluronic acid/gelatin hydrogel [286]. TetraPAc-crosslinked hydrogel presented significantly higher shear storage moduli and better cell proliferation than the PEG diacrylate (PEGDA)-crosslinked gel. The printed structure was biocompatible with high viability for up to 4 weeks under the culture.

With the development of characterization techniques, accurate and vivid imaging of the blood vessel formation can be realized. Mesoscopic fluorescence molecular tomography (MFMT) and micro-CT were reported by Zhao et al. for imaging vascular constructs manufactured via bioprinting and vascular perfusion [287]. The schematic of the fabrication process is shown in Fig. 47 [287]. Briefly, collagen layers were printed first as bottom layers. Then one line with gelatin and human umbilical vein endothelial cells (HUVECs) was printed followed by filling the entire perfusion chamber by collagen. Cells got attached to channels at $37^{\circ} \mathrm{C}$. Then the extra gelatin liquid was removed by perfusion media flow. Both MFMT and micro-CT images showed accurate vascular tissue formation around the channels, which provided options for the imaging of vascular-like constructs with high sensitivity and accuracy. Artificial cardiovascular tissues were also reported using AM techniques. Hockaday et al. reported a 3D printing/photocrosslinking method for the fabrication of an aortic valve graft using poly-ethylene glycol-diacrylate (PEG-DA)/alginate hydrogels [283]. There are three major criteria for a tissue-engineered heart valve, namely geometry, mechanical heterogeneity and physiological function. The reported aortic valve graft showed great shape fidelity and heterogeneous mechanical property with an elastic modulus varying from $5.3 \pm 0.9 \mathrm{kPa}$ to $74.6 \pm 1.5 \mathrm{kPa}$. In addition, porcine aortic valve interstitial cells (PAVIC) were seeded into the scaffolds for the tissue functionalization. The result showed that a nearly $100 \%$ cell viability for up to 21 days was maintained indicating a promising approach to be explored further for aortic valve grafts. 
4.4.3. Skin-Skin is the soft outer covering of the human body composed of multiple layers that protect muscles, ligaments, bones, and other internal organs. The functions of the skin can be separated into many categories including (a) protection: skin acts as the first defense systems against outside pathogens. It is also a barrier to avoid the loss of essential nutrients when under water. (b) Body temperature control: the regulation of body temperature can be conducted by controlling the activity of eccrine glands, and dilation or constriction of the vessel superficial flow. (c) Sensation: skin tissue includes nerve endings for sensations such as temperature, pain, and pressure. The three primary layers constructing skin system are epidermis, dermis, and hypodermis. Epidermis is the outmost layer of the skin, which is responsible for protecting the body against any infection or nutrient/water loss. Dermis locates beneath epidermis containing connective tissues as cushions to withstand the outside stress. The hypodermis is the deepest layer of the skin whose function is to attach muscles and bones together with blood vessels and nerves. The major cell types for the skin are keratinocytes and fibroblasts. Generally, damaged skin tissue can heal itself much faster than other soft tissues. We can wait for the natural healing, but the scar formation after healing is hard to remove. For patients with a large area of the burn injury, the accelerated skin healing rate is necessary to prevent infections. Recently, AM methods have been used for the skin tissue engineering. Both top-down scaffold system or bottom-up bioprinting show great potential for the future clinical use.

Kim et al. reported a study using a system assembly using a 3D plotting and a cryogenic refrigeration platform for skin tissue fabrication [188]. Collagen was extruded from the bioplotter followed by the solidification in the cryogenic refrigeration platform. As received scaffolds were further processed under freeze drying and curing processes to achieve the final scaffold. Structure, porosity and mechanical properties of the scaffold could be manipulated using this novel printing system. In addition, in vitro co-culture experiments using keratinocyte/fibroblast showed the complete cell migration and well differentiation skin tissue within the scaffold. More recent studies started to use cell bioprinting to incorporate cell and material matrix together for skin tissue regeneration. Lee et al. reported a composite of collagen hydrogel precursor, fibroblasts, and keratinocytes for the bioprinting of artificial skin tissues [288]. The entire skin tissue construct started with the printing of one layer of collagen hydrogel precursor and one layer of fibroblast embedded collagen hydrogel precursor for mimicking the epidermis layer of the natural skin. For every interface of different layers, the cross-linking material was used for the curing of the hydrogel. After 6 layers of collagen hydrogel precursors, another layer with keratinocytes embedded collagen hydrogel precursor was added for the construction of the dermis layer. Finally, the entire skin tissue construct was finished with two more layers of collagen precursors. The tissue construct was tested on a 3D skin wound model using poly (dimethylsiloxane) (PDMS). Both cell lines showed high proliferation on horizontal and vertical directions indicating an excellent imitation of natural skin tissue and its potential in clinical use.

Laser assisted bioprinting was also utilized to manufacture artificial skin tissues. It has the advantages for executing sophisticated designs with biomaterial and cells together. Koch et al. reported a study using laser-induced forward transfer (LIFT) for the manufacture of skin tissues [289]. A well-defined pattern was printed using this technique by fibroblasts/ 
keratinocytes and human mesenchymal stem cells (hMSC). In vitro results indicated that the printing process didn't decrease the viability of cells. High cell survivability after printing and unaffected proliferation rate showed the further research for this technique. In a followup study, the same technique was used adding collagen as the matrix material for the generation of skin tissues [290]. The keratinocytes were found to be well distributed within their assigned area. In addition, tissue morphogenesis and cohesion were observed by a scrape-loading method showing an excellent cell diffusion and gap junction coupling. For further evaluation of this technique, Michael et al. reported a laser assisted bioprinting method using fibroblasts/keratinocytes on top of MatridermH, a stabilizing matrix, for in vivo characterizations on the dorsal skin fold chamber of mice [291]. The printed skin graft was directly tested in skin wounds of dorsal skin fold chamber in nude mice for 11 days. Multi-layered epidermis and e-cadherin with blood vessel formation were found near the mice epidermis indicating an excellent healing junction and skin tissue regeneration. In addition, an in vitro control was performed in an air-liquid-interface environment. High cell proliferation and e-cadherin were observed in vitro, but the epidermis formation was not multilayer. This study showed that laser assisted bioprinting can be used for making skin tissue constructs with great skin regeneration ability in vivo.

\subsection{Organ tissue engineering}

The use of AM approachs towards organ regeneration is very complex. However, recently, researchers have started to develop structures using AM specifically for organ regenerations. For example, ear, an important organ for hearing and balancing, has been manufactured by AM technologies. The ear can be divided into three parts, outer ear, middle ear and inner ear. The outer ear has a complex shape and is mainly composed of cartilage and fat tissues. The middle and inner ear constructs the sensing system for balancing and hearing. The manufacturing of the artificial ear is a big challenge due to its complexity in shape and property requirements.

Lee et al. reported a sacrificial layer modified bioprinting method for making the artificial ear using PCL and cell-laden hydrogel [293]. A dual cells system, chondrocytes and adipocytes, were laden in a $4 \%$ alginate hydrogel. The framework of the ear was printed using PCL as the matrix and PEG as the sacrificial material. Then PEG was removed by the distilled water. The maximum tensile modulus was $57.55 \pm 3.1 \mathrm{MPa}$ using a line pitch of $500 \mu \mathrm{m}$. The cell-laden hydrogel was dispensed between the spacing of the framework. Final artificial ear showed enhanced chondrogenesis and adipogenesis in vitro. Another novel bioink printing method using alginate and nanofibrillated cellulose (NFC) with human chondrocytes was also used to print ear [292]. Different bioink compositions were prepared with different concentrations of NFC and alginate. A combination of $80 \mathrm{wt} \%$ of NFC and 20 wt $\%$ alginate was finally selected as the bioink for preparing artificial ears. The discs using this formula had a compressive stiffness of $150 \mathrm{kPa}$ and a compressive strength of $33 \mathrm{kPa}$. The final printed ear had high structural stability and fidelity. In addition, in vitro characterization using human nasoseptal chondrocytes showed high cell viability up to 7 days. Mannoor et al. reported a bionic ear fabrication by cell-laden hydrogel matrix as the outer ear and intertwined silver nanoparticles infused conducting polymer as middle and inner ears, as shown in Fig. 48 [294]. Chondrocytes were laden in alginate hydrogel as the 
matrix material for the outer ear. An inductive coil antenna with silver nanoparticle (AgNP) infusion was connected to cochlea-shaped electrodes embedded in silicone. This bioprinting process was featured with high geometry precision and cell viability after the printing. In addition, with the aid of the electronic sensing system, the printed ear was able to receive electromagnetic signals in a wide range. Such findings unveil other possibilities to combine tissue engineering combined with electronics for making artificial cyborg organs.

Liver is another organ that has drawn lots of interest for AM. Liver is a wedge-shaped organ with four lobes composed of millions of hepatocytes for its basic metabolic functions. Liver failures caused by chronic liver diseases, such as hepatitis, cirrhosis and hemochromatosis, can only be treated through transplantation. However, problems, such as allograft rejections, scarce source of liver donor, and donor mortality, are the main challenges of liver transplantation. One possible solution is to develop tissue engineered liver substitute for liver transplantation. Nevertheless, it is still a challenge to manufacture artificial livers because of its inherent complexity in both geometry and functions.

Huang et al. reported a study using SLS for making interconnected flow-channel constructs for liver tissue regeneration [296]. PCL was used as a matrix material. In order to further increase the interconnected porosity within the matrix, $\mathrm{NaCl}$ particles were added to the PCL as porogen and further removed by the water leaching. The final construct was highly porous with a volume porosity of $89 \%$ and a pore size range between 100 and $200 \mu \mathrm{m}$. For initial liver cell seeding, avidin-biotin binding-based cell seeding method was used. Human hepatoma Hep G2 cells were loaded into the construct during in vitro experiments via perfusion culturing for 9 days. The perfusion culturing system and the new cell seeding method significantly improved the liver tissue regeneration and further functionalization. In another study, a chitosan-gelatin hybrid bionic liver construct with detailed hepatic chambers, flow-channel network, central vein and portal vein design was fabricated using a combination of SLA, microreplication and freeze drying [295]. A resin mold was created by SLA followed by poly-dimethylsiloxane (PDMS) casting in order to get a PDMS mold. Finally, the chitosan-gelatin solution was cast into the PDMS mold followed by freeze drying to achieve the final liver construct. The as fabricated construct showed excellent hydrophilicity and biodegradability when tested in PBS. In addition, hepatocytes were loaded into the artificial liver structure to characterize its biological responses in vitro. Large colonies of hepatocytes were observed all over the hepatic chambers after 7 days. Moreover, the detection of urea synthesis and albumin secretion further proved the suitability of this construct for growing liver tissues. Zein et al. reported a manufacturing process for making a patient liver model with detailed features such as biliary structures and complex networks of vessels for preoperative planning of the liver transplantation [297]. Six patients including three liver donors and three liver recipients were the subjects for this study. The 3D images were created from CT and MRI images. Parts were printed by Connex 350 3D printer using different materials for different regions, as shown in Fig. 49 [297]. The identity of geometrical and anatomical structures between native livers and 3D printed models was further proved by preoperative, intraoperative, and postoperative assessments. Such approaches will offer substantial help for planning liver transplantation surgery in the coming days. Applications for AM of biomaterials are very diverse. Table 9 summarizes the different application areas with various cells, biomaterials and growth factors. 


\section{Current challenges and future trends}

Future trends for the AM of biomaterials are multi-faceted and depend on many factors. So far, most of the progress is limited to research and development, while in some isolated cases commercial products have been developed and used clinically. However, that trend needs to shift more towards innovation in biomedical problem solving using AM followed by successful commercialization. The main hurdle in that pathway is not the technology development or man-power shortage, but regulation. Regulation related to biomedical devices usually lags behind technology development. Therefore, it is natural to see slow progress to shape the regulatory agencies around the world towards devices produced via AM. A key contributor in that slow process is the inherent concerns related to reliability and reproducibility of parts produced via AM. For example, if a Ti6Al4V part is produced via an Arcam's E-beam machine and another one is produced via EOS's Laser melting machine with identical shape, it is natural that properties will be different in those two parts due to the nature of heating and cooling during processing that can result in differences in microstructure as well as residual stresses. Therefore, a key difference in AM produced parts needs to be the minimum performance requirement than the actual measured properties. For example, in Arcam and EOS produced Ti6Al4V parts, in both cases, mechanical properties are expected to be better than the commercially available wrought products due to grain refinement during processing. However, establishing such process-performance criteria takes time and resources. And if it needs to be done for each material or device, it is sometimes the major bottleneck in the commercialization of additively manufactured products. In recent years, most biomedical industries have taken a better approach towards AM based products. Instead of focusing on Class II or Class III devices, various industries are now focusing on Class I devices that are easy to get regulatory approval, yet can be made patient matched, if needed, to enhance surgeon's comfort or produce on demand instead of projection based manufacturing. It is envisioned that a large number of approved Class I devices will also increase confidence amongst the regulatory bodies in the coming days that can help protocol development towards approval of Class II and Class III devices. It is also important to note that US Food and Drug Administration (FDA) has already given several $510 \mathrm{~K}$ approvals to load bearing Class II implants that are now commercially available. Similar trends can also be seen in other regulatory bodies around the world.

Another important area that is expected to dominate in the coming days is multi-materials structures via AM. Unlike other manufacturing processes, AM is not just a shaping tool, but it can also allow manufacturing parts with multiple materials. Functional parts with not just structural variations, but with compositional variations can be fabricated using AM. The critical issue is not on how to make it, but what to make as well. Most designers still use traditional design approaches in which parts with different compositions are designed separately followed by assembly using various techniques. However, AM can potentially minimize many of those assembly requirements by manufacturing parts with multiple compositions in one operation. A simple example could be coated implants for load-bearing applications. Coatings are preferred to enhance bone tissue integration and added onto uncoated implants using different processes. However, AM can be used to produce both, coated or uncoated, implants using the same machine. In fact, AM can be used to produce 
even ceramic coated metal implants in the same operation. Such freedom in a manufacturing operation is expected to cultivate innovation in product design to offer performance that was not possible in the past.

Design for AM is also an area that is expected to grow rapidly in the coming days. Traditional 2D and 3D designs are good for most conventional manufacturing that are either shaping based or subtractive in nature. However, AM can be used to manufacture parts that can be fully enclosed or a combination of multiple moving parts built in one operation or color coded in different areas for different functionality and so on. Most AM operations today are focused on building parts that are designed for conventional manufacturing. Application of AM is reducing the lead time and cost of tooling, which is significant for low volume manufacturing. However, to fully utilize AM's ability, parts can be designed with varying thickness or voids to maximize materials utilization and minimize cost, which can be designed for AM and manufactured using various AM techniques. Such approach is slowly coming and transforming the world of manufacturing. In the coming days, it is envisioned that AM will significantly influence innovative product designs and related performance.

Bioprinting is another area of AM that is making a significant impact. The ability to codeposit both cellular and acellular structures is not trivial, but doable. Organ and tissue engineering fields can benefit tremendously if AM can successfully deposit acellular structures with specific cells in it that can be cultured in a bioreactor. Primary challenges include limited 3D structure fabrication ability, keeping cells viable during processing, on demand printing ability with a variety of materials and cells, and finally understanding design and print strategy to effectively use this ability towards biofabrication for tissue engineering. For clinical application, it may be needed to print scaffolds or tissues in a larger scale than typically produced in a laboratory research setting. The printing speed is a concern for the cost-efficiency of the method, especially for commercialization of clinical tissue or scaffold AM. In addition, for some injuries, if the printing speed is fast enough, the in situ tissue repair is possible to replace dead tissues and regenerate new tissues for repairing. This technique has been applied on skin tissue repair using laser assisted bioprinting in a mouse model [294]. However, there is little development of this technology for other tissue or organ regeneration. Finally, a higher printing resolution may be necessary for complex organ or organ model printing. For transplantation surgeries such as liver transplantation, preoperative planning is very important to increase the survival rate for donors and recipients. Detailed imaging of all internal liver components will help in the planning the surgery procedures.

AM techniques can combine electronic devices for assisting AM prepared artificial organs before those are fully operational. Because of complexity in human organs, such as liver, heart, kidney, still they can't be manufactured with comparable functions as native organs. Even though the bioprinting technology has achieved some success using cells from patients and showed traces of self-functionalization, the full functionalization of bioprinted organs is still a big challenge when thinking about the complex metabolism system, multiple functions and a long time of self-functionalization for each organ. However, some electronic device might help this situation via initially achieving some functions of the organ. At the same 
time, the artificial organ is under self-functionalization until it becomes an organ with comparable functions to native organs. The artificial ear with the inductive coil to receive electromagnetic signals for hearing has been manufactured by bioprinting technique [294]. However, there is still a long way to go to attain cyborg organs with growing functions via cell self-functionalization.

With the development of pharmaceutical technologies, AM has the potential to provide an excellent tool to make biocompatible vessels for delivering drugs to locations affected by the disease. Through the advantage of AM for constructing the complex structure, it is possible to control the release of drugs to improve their effective time and minimize their toxicity. Controlled alendronate release using PCL coating on 3D printed porous TCP scaffolds was achieved by Tarafder et al. [49]. However, there are few reports about drug delivery system fabricated via printing matrix material and drug molecules in one system and control the release by manipulating the structure design and material composition alternation, which is one promising direction for advanced drug delivery system.

Overall, even though a lot of successes have been achieved for AM of biomaterials, it is still in its infancy. Challenges including technical issues for AM technology based on clinical need and biomaterial selections are addressed above. Multidisciplinary research will be necessary to face those challenges and fully realize the potential of AM in biomedical applications in the coming days.

\section{Acknowledgements}

Authors like to acknowledge financial support from National Institute of Arthritis and Musculoskeletal and Skin Diseases of the National Institutes of Health under Award Numbers R01 AR066361-01A1 and R01 AR06730601A1. The content is solely the responsibility of the authors and does not necessarily represent the official views of the National Institutes of Health.

\section{Abbreviations:}

$\begin{array}{ll}\text { AM } & \text { Additive Manufacturing } \\ \text { SFF } & \text { Solid Freeform Fabrication } \\ \text { LM } & \text { Layered Manufacturing } \\ \text { RP } & \text { Rapid Prototyping } \\ \text { CAD } & \text { Computer Aided Design } \\ \text { FDM } & \text { Fused Deposition Modeling } \\ \text { ASTM } & \text { American Society for Testing of Materials } \\ \text { TCP } & \text { Tricalcium Phosphate } \\ \text { PCL } & \text { Polycaprolactone } \\ \text { LENS } & \text { Laser Engineered Net Shaping } \\ \text { EBDM } & \text { Electron Beam Direct Manufacturing }\end{array}$




\begin{tabular}{|c|c|}
\hline $\mathrm{CNC}$ & Computer Numeric Control \\
\hline LBMD & Laser Based Metal Deposition \\
\hline EBAMM ${ }^{\mathrm{TM}}$ & Electron Beam Additive Manufacturing \\
\hline FDC & Fused Deposition of Ceramic \\
\hline PBF & Powder Bed Fusion \\
\hline SLS & Selective Laser Sintering \\
\hline SLM & Selective Laser Melting \\
\hline EBM & Electron Beam Melting \\
\hline SLA & stereolithography \\
\hline DMD & Digital Micromirror Device \\
\hline CaPs & Calcium Phosphates \\
\hline HA & hydroxyapatite \\
\hline BMP-2 & Bone Morphogenetic Protein 2 \\
\hline TTCP & Tetracalcium Phosphate \\
\hline DCP & Dicalcium Phosphate \\
\hline VEGF & Vascular Endothelial Growth Factor \\
\hline CMC & Carboxymethyl Cellulose \\
\hline DLP & Digital Light Processing \\
\hline PVA & Polyvinyl Alcohol \\
\hline $\mathbf{A L P}$ & Alkaline Phosphatase \\
\hline BCP & Biphasic Calcium Phosphate \\
\hline DLF & Direct Laser Fabrication \\
\hline LAM & Laser Additive Manufacturing \\
\hline HIP & Hot Isostatic Pressing \\
\hline DLF & Direct Laser Forming \\
\hline SBF & Simulated Body Fluid \\
\hline PBS & Phosphate Buffer Saline \\
\hline BSA & Bovine Serum Albumin \\
\hline ECM & Extracellular Matrix \\
\hline
\end{tabular}




$\begin{array}{ll}\text { PGA } & \text { Polyglycolic Acid } \\ \text { PLA } & \text { Polylactic Acid } \\ \text { PLGA } & \text { Poly(lactic-co-glycolic acid) } \\ \text { PLLA } & \text { Poly-L-lactide } \\ \text { PDLA } & \text { Poly-D-lactide } \\ \text { PLDLLA } & \text { Poly(L-lactide-co-D,L-lactide) } \\ \text { PEG } & \text { Polyethylene Glycol } \\ \text { SSLS } & \text { Surface Selective Laser Sintering } \\ \text { PPF } & \text { Polypropylene Fumarates } \\ \text { PEEK } & \text { polyetheretherketone } \\ \text { CPP } & \text { Calcium Polyphosphate } \\ \text { THR } & \text { Total Hip Replacement } \\ \text { TKR } & \text { Total Knee Replacement } \\ \text { FGF-2 } & \text { Fibroblast Growth Factor-2 } \\ \text { DMLS } & \text { Direct Metal Laser Sintering }\end{array}$

\section{References}

[1]. F42. 91 Additive Manufacturing Technologies. Standard Terminology for Additive Manufacturing Technologies; 2012.

[2]. Sachs EM. Powder dispensing apparatus using vibration. US6036777 A; 2000.

[3]. Bredt JF, Clark S, Gilchrist G. Three dimensional printing material system and method. US7087109 B2; 2006.

[4]. Tay BY, Evans JRG, Edirisinghe MJ. Solid freeform fabrication of ceramics. Int Mater Rev 2003;48:341-70.

[5]. Liu J, Ryneson ML. Blended powder solid-supersolidus liquid phase sintering. CN1649688 A; 2005.

[6]. Cima LG, Cima MJ. Preparation of medical devices by solid free-form fabrication methods. US5490962 A; 1996.

[7]. Cima M, Sachs E, Fan T, Bredt JF, Michaels SP, Khanuja S, et al. Three-dimensional printing techniques. US5387380 A; 1995.

[8]. Sachs EM, Cima MJ, Caradonna MA, Grau J, Serdy JG, Saxton PC, et al. Jetting layers of powder and the formation of fine powder beds thereby. US6596224 B1; 2003.

[9]. Lu K, Hiser M, Wu W. Effect of particle size on three dimensional printed mesh structures. Powder Technol 2009;192:178-83.

[10]. Liu J, Ryneson ML. Blended powder solid-supersolidus liquid phase sintering. US7070734 B2; 2006.

[11]. Zhou Z, Buchanan F, Mitchell C, Dunne N. Printability of calcium phosphate: calcium sulfate powders for the application of tissue engineered bone scaffolds using the $3 \mathrm{D}$ printing technique. Mater Sci Eng C 2014;38:1-10.

[12]. Bredt JF. Binder composition for use in three dimensional printing. US5851465 A; 1998. 
[13]. Oriakhi C, Lambright TM. Calcium aluminate cement compositions for solid freeform fabrication. US7258736 B2; 2007.

[14]. Bredt JF, Anderson TC, Russell DB. Three dimensional printing material system and method. US6610429 B2; 2003.

[15]. Feenstra FK. Method for making a dental element. EP1207803 A1; 2002.

[16]. Maleksaeedi S, Eng H, Wiria FE, Ha TMH, He Z. Property enhancement of 3D-printed alumina ceramics using vacuum infiltration. J Mater Process Technol 2014;214:1301-6.

[17]. Liu J, Rynerson M. Method for article fabrication using carbohydrate binder. 6585930; 2003.

[18]. Greil P Polymer derived engineering ceramics. Adv Eng Mater 2000;2:339-48.

[19]. Peuckert M, Vaahs T, Brück M. Ceramics from organometallic polymers. Adv Mater 1990;2:398-404.

[20]. Arghavan Farzadi MS-H. Effect of layer thickness and printing orientation on mechanical properties and dimensional accuracy of 3D printed porous samples for bone tissue engineering. PLoS ONE 2014;9:e108252. [PubMed: 25233468]

[21]. Wang H-R, Cima MJ, Kernan BD, Sachs EM. Alumina-doped silica gradient-index (GRIN) lenses by slurry-based three-dimensional printing (S-3DPTM). J Non-Cryst Solids 2004;349:360 7.

[22]. Yasrebi M, Van Ginhoven R, Kemp WW, Schmidt BJ, Sturgis DH. Hydroxylated carboxylic acid monomers as stabilizing agents for yttria-alumina aqueous suspensions. J Am Ceram Soc 2005;88:536-9.

[23]. Suwanprateeb J, Chumnanklang R. Three-dimensional printing of porous polyethylene structure using water-based binders. J Biomed Mater Res B Appl Biomater 2006;78B:138-45.

[24]. Bredt JF, Anderson T. Method of three dimensional printing. US5902441 A; 1999.

[25]. Castilho M, Moseke C, Ewald A, Gbureck U, Groll J, Pires I, et al. Direct 3D powder printing of biphasic calcium phosphate scaffolds for substitution of complex bone defects. Biofabrication 2014;6:015006. [PubMed: 24429776]

[26]. Shen J Material system for use in three dimensional printing. US20020016387 A1; 2002.

[27]. Sachs EM, Hadjiloucas C, Allen S, Yoo HJ. Metal and ceramic containing parts produced from powder using binders derived from salt. US6508980 B1; 2003.

[28]. Pfister A, Landers R, Laib A, Hübner U, Schmelzeisen R, Mülhaupt R. Biofunctional rapid prototyping for tissue-engineering applications: 3D bioplotting versus 3D printing. J Polym Sci Part Polym Chem 2004;42:624-38.

[29]. Koegler WS, Griffith LG. Osteoblast response to PLGA tissue engineering scaffolds with PEO modified surface chemistries and demonstration of patterned cell response. Biomaterials 2004;25:2819-30. [PubMed: 14962560]

[30]. Caradonna MA, Cima MJ, Grau J, Moon J, Sachs EM, Saxton PC, et al. Jetting layers of powder and the formation of fine powder beds thereby. EP1009614 A1; 2000.

[31]. Khoshnevis B Selective inhibition of bonding of power particles for layered fabrication of 3-D objects. US6589471 B1; 2003.

[32]. Almquist TA, Hull CW, Thayer JS, Leyden RN, Jacobs PF, Smalley DR. Rapid recoating of three-dimensional objects formed on a cross-sectional basis. US5902537 A; 1999.

[33]. Gaylo CM, Imiolek IJ. Method for dispensing of powders. US5934343 A; 1999.

[34]. Bynum DK. Automated manufacturing system using thin sections. US5088047 A; 1992.

[35]. Sachs EM, Haggerty JS, Cima MJ, Williams PA. Three-dimensional printing techniques. US5204055 A; 1993.

[36]. Pond SF. Inkjet technology and product development strategies. Carlsbad, CA: Torrey Pines Research; 2000.

[37]. Utela B, Storti D, Anderson R, Ganter M. A review of process development steps for new material systems in three dimensional printing (3DP). J Manuf Process 2008;10:96-104.

[38]. Sachs E, Curodeau A, Fan T, Bredt JF, Cima M, Brancazio D. Three dimensional printing system. US5807437 A; 1998.

[39]. Utela BR, Storti D, Anderson RL, Ganter M. Development process for custom three-dimensional printing (3DP) material systems. J Manuf Sci Eng 2010;132:011008. 
[40]. Monkhouse DC, Kumar S, Rowe CW, Yoo J. Rapid prototyping and manufacturing process. US6547994 B1; 2003.

[41]. Gonzalez JA, Mireles J, Lin Y, Wicker RB. Characterization of ceramic components fabricated using binder jetting additive manufacturing technology. Ceram Int 2016;42(9):10559-64.

[42]. Fielding GA, Bandyopadhyay A, Bose S. Effects of silica and zinc oxide doping on mechanical and biological properties of 3D printed tricalcium phosphate tissue engineering scaffolds. Dent Mater 2012;28:113-22. [PubMed: 22047943]

[43]. Cao S, Wei X-F, Sun Z-J, Zhang H-H. Investigation on urea-formaldehyde resin as an in-powder adhesive for the fabrication of $\mathrm{Al}_{2} \mathrm{O}_{3}$ /borosilicate-glass composite parts by three dimensional printing (3DP). J Mater Process Technol 2015;217:241-52.

[44]. Tarafder S, Davies NM, Bandyopadhyay A, Bose S. 3D printed tricalcium phosphate scaffolds: effect of $\mathrm{SrO}$ and $\mathrm{MgO}$ doping on in vivo osteogenesis in a rat distal femoral defect model. Biomater Sci 2013;1:1250-9. [PubMed: 24729867]

[45]. Sachs EM, Cima MJ, Bredt JF, Khanuja S. Process for removing loose powder particles from interior passages of a body. US5490882 A; 1996.

[46]. Sachs E, Michaels SP, Allen SM. Enhancement of thermal properties of tooling made by solid free form fabrication techniques. US5775402 A; 1998.

[47]. Tarafder S, Balla VK, Davies NM, Bandyopadhyay A, Bose S. Microwave-sintered 3D printed tricalcium phosphate scaffolds for bone tissue engineering. J Tissue Eng Regen Med 2013;7:63141. [PubMed: 22396130]

[48]. Ke D, Dernell W, Bandyopadhyay A, Bose S. Doped tricalcium phosphate scaffolds by thermal decomposition of naphthalene: mechanical properties and in vivo osteogenesis in a rabbit femur model. J Biomed Mater Res B Appl Biomater 2015;103:1549-59. [PubMed: 25504889]

[49]. Tarafder S, Bose S. Polycaprolactone-coated 3D printed tricalcium phosphate scaffolds for bone tissue engineering: in vitro alendronate release behavior and local delivery effect on in vivo osteogenesis. ACS Appl Mater Interfaces 2014;6:9955-65. [PubMed: 24826838]

[50]. Moon J, Caballero AC, Hozer L, Chiang Y-M, Cima MJ. Fabrication of functionally graded reaction infiltrated $\mathrm{SiC}-\mathrm{Si}$ composite by three-dimensional printing (3DPTM) process. Mater Sci Eng A 2001;298:110-9.

[51]. Ayers JD, Schaefer RJ, Robey WP. A laser processing technique for improving the wear resistance of metals. JOM 2013;33:19-23.

[52]. Han L, Phatak KM, Liou FW. Modeling of laser cladding with powder injection. Metall Mater Trans B 2004;35:1139-50.

[53]. Abioye TE, Folkes J, Clare AT. A parametric study of Inconel 625 wire laser deposition. J Mater Process Tech 2013;213(12):2145-51.

[54]. Justin DF, Stucker BE. Laser based metal deposition (LBMD) of implant structures. US7632575 B2; 2009.

[55]. Sahasrabudhe H, Bandyopadhyay A. Additive manufacturing of reactive in situ Zr based ultrahigh temperature ceramic composites. JOM 2016;68:822-30.

[56]. Kobryn PA, Semiatin SL. The laser additive manufacture of Ti-6Al-4V. JOM 2001;53:40-2.

[57]. Abbas G, West D. Laser surface cladding of stellite and stellite-SiC composite deposits for enhanced hardness and wear. Wear 1991;143:353-63.

[58]. Pei YT, Ocelik V, De Hosson JTM. SiCp/Ti6Al4V functionally graded materials produced by laser melt injection. Acta Mater 2002;50:2035-51.

[59]. Vreeling JA, Ocelík V, De Hosson JTM. Ti-6Al-4V strengthened by laser melt injection of WC p particles. Acta Mater 2002;50:4913-24.

[60]. Zhang Y, Wei Z, Shi L, Xi M. Characterization of laser powder deposited Ti-TiC composites and functional gradient materials. J Mater Process Technol 2008;206:438-44.

[61]. Krishna BV, Bose S, Bandyopadhyay A. Low stiffness porous Ti structures for load-bearing implants. Acta Biomater 2007;3:997-1006. [PubMed: 17532277]

[62]. Vamsi Krishna B, Xue W, Bose S, Bandyopadhyay A. Functionally graded Co-Cr-Mo coating on Ti-6Al-4V alloy structures. Acta Biomater 2008;4:697-706. [PubMed: 18054298] 
[63]. Balla VK, Devasconcellos PD, Xue W, Bose S, Bandyopadhyay A. Fabrication of compositionally and structurally graded $\mathrm{Ti}-\mathrm{TiO}_{2}$ structures using laser engineered net shaping (LENS). Acta Biomater 2009;5:1831-7. [PubMed: 19233752]

[64]. Dittrick S, Balla VK, Davies NM, Bose S, Bandyopadhyay A. In vitro wear rate and Co ion release of compositionally and structurally graded CoCrMo-Ti6Al4V structures. Mater Sci Eng C 2011;31:809-14.

[65]. Sahasrabudhe H, Harrison R, Carpenter C, Bandyopadhyay A. Stainless steel to titanium bimetallic structure using LENS ${ }^{\mathrm{TM}}$. Addit Manuf 2015;5:1-8.

[66]. Crump SS. Apparatus and method for creating three-dimensional objects. US5121329 A; 1992.

[67]. Nikzad M, Masood SH, Sbarski I. Thermo-mechanical properties of a highly filled polymeric composites for fused deposition modeling. Mater Design 2011;32(6):3448-56.

[68]. Bandyopadhyay A, Panda RK, Janas VF, Agarwala MK, Danforth SC, Safari A. Processing of piezocomposites by fused deposition technique. J Am Ceram Soc 1997;80:1366-72.

[69]. Bose S, Suguira S, Bandyopadhyay A. Processing of controlled porosity ceramic structures via fused deposition. Scr Mater 1999;41:1009-14.

[70]. Stansbury JW, Idacavage MJ. 3D printing with polymers: Challenges among expanding options and opportunities. Dent Mater 2016;32(1):54-64. [PubMed: 26494268]

[71]. Gibson I, Rosen DW, Stucker B. Additive manufacturing technologies. Boston, MA: Springer; 2010.

[72]. Meiners W, Wissenbach KD, Gasser AD. Shaped body especially prototype or replacement part production. DE19649865 C1; 1998.

[73]. Bandyopadhyay A, Bose S, Das S. 3D printing of biomaterials. MRS Bull 2015;40:108-15.

[74]. Bhavar V, Kattire P, Patil V, Khot S, Gujar K, Singh R. A review on powder bed fusion technology of metal additive manufacturing In: 4th Int. Conf. Exhib. Addit. Manuf. Technol, Banglore, India; 2014.

[75]. Mani M, Lane B, Donmez A, Feng S, Moylan S, Fesperman R. Measurement science needs for real-time control of additive manufacturing powder bed fusion processes. National Institute of Standards and Technology; 2015.

[76]. Vayre B, Vignat F, Villeneuve F. Metallic additive manufacturing: state-of-the-art review and prospects. Mech Ind 2012;13:89-96.

[77]. Loeber L, Biamino S, Ackeli U, Sabbadini S, Epicoco P, Fino P, et al. Comparison of selective laser and electron beam melted titanium aluminides In: Proc. Solid Free. Fabr. Symp. Austin TX USA; 2011 p. 8-10.

[78]. Melchels FPW, Feijen J, Grijpma DW. A review on stereolithography and its applications in biomedical engineering. Biomaterials 2010;31:6121-30. [PubMed: 20478613]

[79]. Crivello JV, Reichmanis E. Photopolymer materials and processes for advanced technologies. Chem Mater 2014;26:533-48.

[80]. Crivello JV, Dietliker K. Photoinitiators for free radical cationic and anionic photopolymerisation. 2nd ed. Chichester; New York: John Wiley \& Sons Inc; 1999.

[81]. Hinczewski C, Corbel S, Chartier T. Ceramic suspensions suitable for stereolithography. J Eur Ceram Soc 1998;18:583-90.

[82]. Brady GA, Halloran JW. Stereolithography of ceramic suspensions. Rapid Prototyp J 1997;3:615.

[83]. Griffith ML, Halloran JW. Ultraviolet curing of highly loaded ceramic suspensions for stereolithography of ceramics. Manuf Sci Eng 1994;68-2:529-34.

[84]. Wu H, Li D, Guo N. Fabrication of integral ceramic mold for investment casting of hollow turbine blade based on stereolithography. Rapid Prototyp J 2009;15:232-7.

[85]. Wu H, Li D, Tang Y, Sun B, Xu D. Rapid fabrication of alumina-based ceramic cores for gas turbine blades by stereolithography and gelcasting. J Mater Process Technol 2009;209:5886-91.

[86]. Greco A, Licciulli A, Maffezzoli A. Stereolitography of ceramic suspensions. J Mater Sci 2001;36:99-105.

[87]. Corcione CE, Greco A, Montagna F, Licciulli A, Maffezzoli A. Silica moulds built by stereolithography. J Mater Sci 2005;40:4899-904. 
[88]. Dufaud O, Marchal P, Corbel S. Rheological properties of PZT suspensions for stereolithography. J Eur Ceram Soc 2002;22:2081-92.

[89]. Dufaud O, Corbel S. Stereolithography of PZT ceramic suspensions. Rapid Prototyp J 2002;8:83-90.

[90]. Kim JY, Lee JW, Lee S-J, Park EK, Kim S-Y, Cho D-W. Development of a bone scaffold using HA nanopowder and micro-stereolithography technology. Microelectron Eng 2007;84:1762-5.

[91]. Corcione CE, Montagna F, Greco A, Licciulli A, Maffezzoli A. Free form fabrication of silica moulds for aluminium casting by stereolithography. Rapid Prototyp J 2006;12:184-8.

[92]. Zimbeck W, Pope M, Rice RW. Microstructures and strengths of metals and ceramics made by photopolymer-based rapid prototyping In: Bourell DL, Beaman JJ, Marcus HL, Crawford RH, Barlow JW, editors. Solid Free. Fabr. Proc. Sept. 1996, Austin, TX: Univ Texas Austin; 1996 p. 411-8.

[93]. Lee K-S, Kim RH, Yang D-Y, Park SH. Advances in 3D nano/microfabrication using two-photon initiated polymerization. Prog Polym Sci 2008;33:631-81.

[94]. Bose S, Tarafder S. Calcium phosphate ceramic systems in growth factor and drug delivery for bone tissue engineering: a review. Acta Biomater 2012;8:1401-21. [PubMed: 22127225]

[95]. Ravaglioli A, Krajewski A. Bioceramics: materials, properties, applications. Houten, Netherlands: Springer Media B.V; 1992.

[96]. Liu H, Yazici H, Ergun C, Webster TJ, Bermek H. An in vitro evaluation of the $\mathrm{Ca} / \mathrm{P}$ ratio for the cytocompatibility of nano-to-micron particulate calcium phosphates for bone regeneration. Acta Biomater 2008;4:1472-9. [PubMed: 18394980]

[97]. Bandyopadhyay A, Bernard S, Xue W, Bose S. Calcium phosphate-based resorbable ceramics: influence of $\mathrm{MgO}, \mathrm{ZnO}$, and $\mathrm{SiO}_{2}$ dopants. J Am Ceram Soc 2006;89:2675-88.

[98]. Griffith ML, Chu T, Wagner W, Halloran JW. Ceramic stereolithography for investment casting and biomedical applications In: Solid free fabr symp proc, vol. 6; 1996 p. 31-8.

[99]. Chu T-MG, Halloran JW, Hollister SJ, Feinberg SE. Hydroxyapatite implants with designed internal architecture. J Mater Sci Mater Med 2001;12:471-8. [PubMed: 15348260]

[100]. Woesz A, Rumpler M, Stampfl J, Varga F, Fratzl-Zelman N, Roschger P, et al. Towards bone replacement materials from calcium phosphates via rapid prototyping and ceramic gelcasting. Mater Sci Eng C 2005;25:181-6.

[101]. Shuai C, Gao C, Nie Y, Hu H, Zhou Y, Peng S. Structure and properties of nano-hydroxypatite scaffolds for bone tissue engineering with a selective laser sintering system. Nanotechnology 2011;22:285703. [PubMed: 21642759]

[102]. Seitz H, Rieder W, Irsen S, Leukers B, Tille C. Three-dimensional printing of porous ceramic scaffolds for bone tissue engineering. J Biomed Mater Res Part B-Appl Biomater 2005;74B:7828.

[103]. Leukers B, Gülkan H, Irsen SH, Milz S, Tille C, Schieker M, et al. Hydroxyapatite scaffolds for bone tissue engineering made by 3D printing. J Mater Sci Mater Med 2005;16:1121-4. [PubMed: 16362210]

[104]. Miranda P, Pajares A, Saiz E, Tomsia AP, Guiberteau F. Fracture modes under uniaxial compression in hydroxyapatite scaffolds fabricated by robocasting. J Biomed Mater Res A 2007;83:646-55. [PubMed: 17508415]

[105]. Dellinger JG, Eurell JAC, Jamison RD. Bone response to 3D periodic hydroxyapatite scaffolds with and without tailored microporosity to deliver bone morphogenetic protein 2 . J Biomed Mater Res A 2006;76A:366-76.

[106]. Wiltfang J, Merten HA, Schlegel KA, Schultze-Mosgau S, Kloss FR, Rupprecht S, et al. Degradation characteristics of alpha and beta tricalcium-phosphate (TCP) in minipigs. J Biomed Mater Res 2002;63:115-21. [PubMed: 11870643]

[107]. Vorndran E, Klarner M, Klammert U, Grover LM, Patel S, Barralet JE, et al. 3D powder printing of $\beta$-tricalcium phosphate ceramics using different strategies. Adv Eng Mater 2008;10:B67-71.

[108]. Felzmann R, Gruber S, Mitteramskogler G, Tesavibul P, Boccaccini AR, Liska R, et al. Lithography-based additive manufacturing of cellular ceramic structures. Adv Eng Mater 2012;14:1052-8. 
[109]. Bian W, Li D, Lian Q, Zhang W, Zhu L, Li X, et al. Design and fabrication of a novel porous implant with pre-set channels based on ceramic stereolithography for vascular implantation. Biofabrication 2011;3:034103. [PubMed: 21725148]

[110]. Yuan J, Zhen P, Zhao H, Chen K, Li X, Gao M, et al. The preliminary performance study of the 3D printing of a tricalcium phosphate scaffold for the loading of sustained release antituberculosis drugs. J Mater Sci 2014;50:2138-47.

[111]. Bose S, Darsell J, Kintner M, Hosick H, Bandyopadhyay A. Pore size and pore volume effects on alumina and TCP ceramic scaffolds. Mater Sci Eng C 2003;23:479-86.

[112]. Limpanuphap S, Derby B. Manufacture of biomaterials by a novel printing process. J Mater Sci Mater Med 2002;13:1163-6. [PubMed: 15348660]

[113]. Moseke C, Gbureck U. Tetracalcium phosphate: synthesis, properties and biomedical applications. Acta Biomater 2010;6:3815-23. [PubMed: 20438869]

[114]. Klammert U, Gbureck U, Vorndran E, Rödiger J, Meyer-Marcotty P, Kübler AC. 3D powder printed calcium phosphate implants for reconstruction of cranial and maxillofacial defects. J Cranio-Maxillofac Surg 2010;38:565-70.

[115]. Habibovic P, Gbureck U, Doillon CJ, Bassett DC, van Blitterswijk CA, Barralet JE. Osteoconduction and osteoinduction of low-temperature 3D printed bioceramic implants. Biomaterials 2008;29:944-53. [PubMed: 18055009]

[116]. Gbureck U, Hölzel T, Doillon CJ, Müller FA, Barralet JE. Direct printing of bioceramic implants with spatially localized angiogenic factors. Adv Mater 2007;19:795-800.

[117]. Gbureck U, Vorndran E, Müller FA, Barralet JE. Low temperature direct 3D printed bioceramics and biocomposites as drug release matrices. J Control Release Off J Control Release Soc 2007;122:173-80.

[118]. Oonishi H, Kushitani S, Yasukawa E, Iwaki H, Hench LL, Wilson J, et al. Particulate bioglass compared with hydroxyapatite as a bone graft substitute. Clin Orthop 1997:316-25. [PubMed: 9005929]

[119]. Xynos ID, Edgar AJ, Buttery LDK, Hench LL, Polak JM. Gene-expression profiling of human osteoblasts following treatment with the ionic products of Bioglass ${ }^{\circledR} 45 \mathrm{~S} 5$ dissolution. J Biomed Mater Res 2001;55:151-7. [PubMed: 11255166]

[120]. Eqtesadi S, Motealleh A, Miranda P, Lemos A, Rebelo A, Ferreira JMF. A simple recipe for direct writing complex 45S5 Bioglass ${ }^{\circledR}$ 3D scaffolds. Mater Lett 2013;93:68-71.

[121]. Eqtesadi S, Motealleh A, Miranda P, Pajares A, Lemos A, Ferreira JMF. Robocasting of 45S5 bioactive glass scaffolds for bone tissue engineering. J Eur Ceram Soc 2014;34:107-18.

[122]. Lusquiños F, del Val J, Arias-González F, Comesaña R, Quintero F, Riveiro A, et al. Bioceramic 3D implants produced by laser assisted additive manufacturing. Phys Proc 2014;56:309-16.

[123]. Tesavibul P, Felzmann R, Gruber S, Liska R, Thompson I, Boccaccini AR, et al. Processing of 45S5 Bioglass $®$ by lithography-based additive manufacturing. Mater Lett 2012;74:81-4.

[124]. Gmeiner R, Mitteramskogler G, Stampfl J, Boccaccini AR. Stereolithographic ceramic manufacturing of high strength bioactive glass. Int J Appl Ceram Technol 2015;12:38-45.

[125]. Wu C, Luo Y, Cuniberti G, Xiao Y, Gelinsky M. Three-dimensional printing of hierarchical and tough mesoporous bioactive glass scaffolds with a controllable pore architecture, excellent mechanical strength and mineralization ability. Acta Biomater 2011;7:2644-50. [PubMed: 21402182]

[126]. Liu X, Rahaman MN, Fu Q. Bone regeneration in strong porous bioactive glass (13-93) scaffolds with an oriented microstructure implanted in rat calvarial defects. Acta Biomater 2013;9:4889-98. [PubMed: 22922251]

[127]. Brown RF, Day DE, Day TE, Jung S, Rahaman MN, Fu Q. Growth and differentiation of osteoblastic cells on 13-93 bioactive glass fibers and scaffolds. Acta Biomater 2008;4:387-96. [PubMed: 17768097]

[128]. Velez M, Jung S, Kolan KCR, Leu MC, Day DE, Chu T-Mg. In vivo evaluation of 13-93 bioactive glass scaffolds made by Selective Laser Sintering (SLS) In: Narayan R, Bose S, Bandyopadhyay A, editors. Biomater Sci Process Prop Appl II, Chichester; New York: John Wiley \& Sons, Inc.; 2012 p. 91-9. 
[129]. Kolan KCR, Leu MC, Hilmas GE, Brown RF, Velez M. Fabrication of 13-93 bioactive glass scaffolds for bone tissue engineering using indirect selective laser sintering. Biofabrication 2011;3:025004. [PubMed: 21636879]

[130]. Kolan KCR, Thomas A, Leu MC, Hilmas G. In vitro assessment of laser sintered bioactive glass scaffolds with different pore geometries. Rapid Prototyp J 2015;21:152-8.

[131]. Detsch R, Schaefer S, Deisinger U, Ziegler G, Seitz H, Leukers B. In vitro: osteoclastic activity studies on surfaces of 3D printed calcium phosphate scaffolds. J Biomater Appl 2011;26:359-80. [PubMed: 20659962]

[132]. Khalyfa A, Vogt S, Weisser J, Grimm G, Rechtenbach A, Meyer W, et al. Development of a new calcium phosphate powder-binder system for the $3 \mathrm{D}$ printing of patient specific implants. J Mater Sci Mater Med 2007;18:909-16. [PubMed: 17216579]

[133]. Bergmann C, Lindner M, Zhang W, Koczur K, Kirsten A, Telle R, et al. 3D printing of bone substitute implants using calcium phosphate and bioactive glasses. J Eur Ceram Soc 2010;30:2563-7.

[134]. Suwanprateeb J, Sanngam R, Suvannapruk W, Panyathanmaporn T. Mechanical and in vitro performance of apatite-wollastonite glass ceramic reinforced hydroxyapatite composite fabricated by 3D-printing. J Mater Sci Mater Med 2009;20:1281-9. [PubMed: 19225870]

[135]. Bose S, Tarafder S, Banerjee SS, Davies NM, Bandyopadhyay A. Understanding in vivo response and mechanical property variation in $\mathrm{MgO}, \mathrm{SrO}$ and $\mathrm{SiO}_{2}$ doped $\beta$-TCP. Bone 2011;48:1282-90. [PubMed: 21419884]

[136]. Bose S, Fielding G, Tarafder S, Bandyopadhyay A. Understanding of dopant-induced osteogenesis and angiogenesis in calcium phosphate ceramics. Trends Biotechnol 2013;31:594605. [PubMed: 24012308]

[137]. Kalita Samar J, Ferguson Melissa. Fabrication of 3-D porous $\mathrm{Mg} / \mathrm{Zn}$ doped tricalcium phosphate bone-scaffolds via the fused deposition modelling. Am J Biochem Biotechnol 2006;2:57-60.

[138]. Tarafder S, Dernell WS, Bandyopadhyay A, Bose S. SrO- and MgO-doped microwave sintered 3D printed tricalcium phosphate scaffolds: mechanical properties and in vivo osteogenesis in a rabbit model. J Biomed Mater Res B Appl Biomater 2015;103:679-90. [PubMed: 25045131]

[139]. Wu X, Mei J. Near net shape manufacturing of components using direct laser fabrication technology. J Mater Process Tech 2003;135:266-70.

[140]. Kelly SM, Kampe SL. Microstructural evolution in laser-deposited multilayer Ti-M-4V builds: Part I. Microstructural characterization. Metall Mater Trans-Phys Metall Mater Sci 2004;35A: 1861-7.

[141]. Xue W, Krishna BV, Bandyopadhyay A, Bose S. Processing and biocompatibility evaluation of laser processed porous titanium. Acta Biomater 2007;3:1007-18. [PubMed: 17627910]

[142]. Heinl P, Rottmair A, Körner C, Singer RF. Cellular titanium by selective electron beam melting. Adv Eng Mater 2007;9:360-4.

[143]. Harrysson O, CansiZoglu O, Marcellin-Little DJ, Cormier DR, West HA. Direct metal fabrication of titanium implants with tailored materials and mechanical properties using electron beam melting technology. Mater Sci Eng C-Biomim Supramol Syst 2008;28:366-73.

[144]. Murr LE, Esquivel EV, Quinones SA, Gaytan SM, Lopez MI, Martinez EY, et al. Microstructures and mechanical properties of electron beam-rapid manufactured Ti-6Al-4V biomedical prototypes compared to wrought Ti-6Al-4V. Mater Charact 2009;60:96-105.

[145]. Parthasarathy J, Starly B, Raman S, Christensen A. Mechanical evaluation of porous titanium (Ti6Al4V) structures with electron beam melting (EBM). J Mech Behav Biomed Mater 2010;3:249-59. [PubMed: 20142109]

[146]. Cheng XY, Li SJ, Murr LE, Zhang ZB, Hao YL, Yang R, et al. Compression deformation behavior of Ti-6Al-4V alloy with cellular structures fabricated by electron beam melting. J Mech Behav Biomed Mater 2012;16:153-62. [PubMed: 23182384]

[147]. Das S, Wohlert M, Beaman JJ, Bourell DL. Processing of titanium net shapes by SLS/HIP. Mater Des 1999;20:115-21.

[148]. Fischer P, Romano V, Weber HP, Karapatis NP, Boillat E, Glardon R. Sintering of commercially pure titanium powder with a Nd:YAG laser source. Acta Mater 2003;51:1651-62. 
[149]. Hollander DA, Von Walter M, Wirtz T, Sellei R, Schmidt-Rohlfing B, Paar O, et al. Structural, mechanical and in vitro characterization of individually structured $\mathrm{Ti}-6 \mathrm{Al}-4 \mathrm{~V}$ produced by direct laser forming. Biomaterials 2006;27:955-63. [PubMed: 16115681]

[150]. Kumar S, Kruth J-P. Wear performance of SLS/SLM materials. Adv Eng Mater 2008;10:750-3.

[151]. Shishkovsky IV, Volova LT, Kuznetsov MV, Morozov YG, Parkin IP. Porous biocompatible implants and tissue scaffolds synthesized by selective laser sintering from Ti and NiTi. J Mater Chem 2008;18:1309-17.

[152]. Wang Y, Shen Y, Wang Z, Yang J, Liu N, Huang W. Development of highly porous titanium scaffolds by selective laser melting. Mater Lett 2010;64:674-6.

[153]. Pattanayak DK, Fukuda A, Matsushita T, Takemoto M, Fujibayashi S, Sasaki K, et al. Bioactive Ti metal analogous to human cancellous bone: fabrication by selective laser melting and chemical treatments. Acta Biomater 2011;7:1398-406. [PubMed: 20883832]

[154]. Gu D, Hagedorn Y-C, Meiners W, Meng G, Batista RJS, Wissenbach K, et al. Densification behavior, microstructure evolution, and wear performance of selective laser melting processed commercially pure titanium. Acta Mater 2012;60:3849-60.

[155]. Sun J, Yang Y, Wang D. Mechanical properties of a Ti6Al4V porous structure produced by selective laser melting. Mater Des 2013;49:545-52.

[156]. Li JP, Habibovic P, van Den Doel M, Wilson CE, de Wijn JR, van Blitterswijk CA, et al. Bone ingrowth in porous titanium implants produced by 3D fiber deposition. Biomaterials 2007;28:2810-20. [PubMed: 17367852]

[157]. Ryan GE, Pandit AS, Apatsidis DP. Porous titanium scaffolds fabricated using a rapid prototyping and powder metallurgy technique. Biomaterials 2008;29:3625-35. [PubMed: 18556060]

[158]. Senthil Selvan J, Subramanian K, Nath AK, Kumar H, Ramachandra C, Ravindranathan SP. Laser boronising of Ti-6Al-4V as a result of laser alloying with pre-placed BN. Mater Sci Eng A 1999;260:178-87.

[159]. Man HC, Zhang S, Cheng FT, Yue TM. Microstructure and formation mechanism of in situ synthesized TiC/Ti surface MMC on Ti-6Al-4V by laser cladding. Scr Mater 2001;44:2801-7.

[160]. Das M, Balla VK, Basu D, Manna I, Sampath Kumar TS, Bandyopadhyay A. Laser processing of in situ synthesized TiB-TiN-reinforced Ti6Al4V alloy coatings. Scr Mater 2012;66:578-81.

[161]. Das M, Bhattacharya K, Dittrick SA, Mandal C, Balla VK, Sampath Kumar TS, et al. In situ synthesized TiB-TiN reinforced Ti6Al4V alloy composite coatings: microstructure, tribological and in-vitro biocompatibility. J Mech Behav Biomed Mater 2014;29:259-71. [PubMed: 24121827]

[162]. Liu W, Dupont JN. Fabrication of functionally graded TiC/Ti composites by laser engineered net shaping. Scr Mater 2003;48:1337-42.

[163]. Das M, Balla VK, Basu D, Bose S, Bandyopadhyay A. Laser processing of SiC-particlereinforced coating on titanium. Scr Mater 2010;63:438-41.

[164]. Das M, Bysakh S, Basu D, Sampath Kumar TS, Balla VK, Bose S, et al. Microstructure, mechanical and wear properties of laser processed $\mathrm{SiC}$ particle reinforced coatings on titanium. Surf Coat Technol 2011;205:4366-73.

[165]. Zhang Y, Sahasrabudhe H, Bandyopadhyay A. Additive manufacturing of Ti-Si-N ceramic coatings on titanium. Appl Surf Sci 2015;346:428-37.

[166]. Yaping Z, Jiacheng G, Jifu T, Zhirong Z. Laser surface coating of a bioceramic composite layer. Surf Coat Technol 1993;58:125-7.

[167]. Lusquiños F, Pou J, Arias JL, Boutinguiza M, León B, Pérez-Amor M, et al. Alloying of hydroxyapatite onto Ti6Al-4V by high power laser irradiation. J Mater Sci Mater Med 2002;13:601-5. [PubMed: 15348591]

[168]. Zheng M, Fan D, Li X-K, Li W-F, Liu Q-B, Zhang J-B. Microstructure and osteoblast response of gradient bioceramic coating on titanium alloy fabricated by laser cladding. Appl Surf Sci 2008;255:426-8.

[169]. Roy M, Vamsi Krishna B, Bandyopadhyay A, Bose S. Laser processing of bioactive tricalcium phosphate coating on titanium for load-bearing implants. Acta Biomater 2008;4:324-33. [PubMed: 18039597] 
[170]. Roy M, Balla VK, Bandyopadhyay A, Bose S. Compositionally graded hydroxyapatite/ tricalcium phosphate coating on Ti by laser and induction plasma. Acta Biomater 2011;7:866-73. [PubMed: 20854939]

[171]. Draper CW, Poate JM. Laser surface alloying. Int Met Rev 1985;30:85-108.

[172]. Walker A, Folkes J, Steen WM, Wes DRF. Laser surface alloying of titanium substrates with carbon and nitrogen. Surf Eng 1985;1:23-9.

[173]. Balla VK, Banerjee S, Bose S, Bandyopadhyay A. Direct laser processing of a tantalum coating on titanium for bone replacement structures. Acta Biomater 2010;6:2329-34. [PubMed: 19931654]

[174]. Dittrick S, Balla VK, Bose S, Bandyopadhyay A. Wear performance of laser processed tantalum coatings. Mater Sci Eng C 2011;31:1832-5.

[175]. Roy M, Balla VK, Bandyopadhyay A, Bose S. MgO-doped tantalum coating on Ti: microstructural study and biocompatibility evaluation. ACS Appl Mater Interfaces 2012;4:57780. [PubMed: 22248182]

[176]. Vrancken B, Thijs L, Kruth J-P, Van Humbeeck J. Microstructure and mechanical properties of a novel $\beta$ titanium metallic composite by selective laser melting. Acta Mater 2014;68:150-8.

[177]. Zhang S, Cheng X, Yao Y, Wei Y, Han C, Shi Y, et al. Porous niobium coatings fabricated with selective laser melting on titanium substrates: preparation, characterization, and cell behavior. Mater Sci Eng C 2015;53:50-9.

[178]. Zhong M, Liu W, Yao K, Goussain J-C, Mayer C, Becker A. Microstructural evolution in high power laser cladding of Stellite 6+WC layers. Surf Coat Technol 2002;157:128-37.

[179]. Xu G, Kutsuna M, Liu Z, Sun L. Characteristic behaviours of clad layer by a multi-layer laser cladding with powder mixture of Stellite- 6 and tungsten carbide. Surf Coat Technol 2006;201:3385-92.

[180]. Janaki Ram GD, Esplin CK, Stucker BE. Microstructure and wear properties of LENS deposited medical grade CoCrMo. J Mater Sci Mater Med 2008;19:2105-11. [PubMed: 17990083]

[181]. Gaytan SM, Murr LE, Martinez E, Martinez JL, Machado BI, Ramirez DA, et al. Comparison of microstructures and mechanical properties for solid and mesh cobalt-base alloy prototypes fabricated by electron beam melting. Metall Mater Trans A 2010;41:3216-27.

[182]. Jevremovic D, Kojic V, Bogdanovic G, Puskar T, Eggbeer D, Thomas D, et al. A selective laser melted $\mathrm{Co}-\mathrm{Cr}$ alloy used for the rapid manufacture of removable partial denture frameworks initial screening of biocompatibility. J Serbian Chem Soc 2011;76:43-52.

[183]. Monroy K, Delgado J, Ciurana J. Study of the pore formation on CoCrMo alloys by selective laser melting manufacturing process. Proc Eng 2013;63:361-9.

[184]. Hedberg YS, Qian B, Shen Z, Virtanen S, Odnevall Wallinder I. In vitro biocompatibility of CoCrMo dental alloys fabricated by selective laser melting. Dent Mater 2014;30:525-34. [PubMed: 24598762]

[185]. Al Jabbari YS, Koutsoukis T, Barmpagadaki X, Zinelis S. Metallurgical and interfacial characterization of PFM Co-Cr dental alloys fabricated via casting, milling or selective laser melting. Dent Mater 2014;30:e79-88. [PubMed: 24534375]

[186]. Takaichi A, Suyalatu T, Nakamoto N, Joko N, Nomura Y, Tsutsumi S, et al. Microstructures and mechanical properties of Co-29Cr-6Mo alloy fabricated by selective laser melting process for dental applications. J Mech Behav Biomed Mater 2013;21:67-76. [PubMed: 23500549]

[187]. Huang S, Fu X. Naturally derived materials-based cell and drug delivery systems in skin regeneration. J Controlled Release 2010;142:149-59.

[188]. Kim G, Ahn S, Yoon H, Kim Y, Chun W. A cryogenic direct-plotting system for fabrication of 3D collagen scaffolds for tissue engineering. J Mater Chem 2009;19:8817-23.

[189]. Lee Y-B, Polio S, Lee W, Dai G, Menon L, Carroll RS, et al. Bio-printing of collagen and VEGF-releasing fibrin gel scaffolds for neural stem cell culture. Exp Neurol 2010;223:645-52. [PubMed: 20211178]

[190]. Sachlos E, Reis N, Ainsley C, Derby B, Czernuszka JT. Novel collagen scaffolds with predefined internal morphology made by solid freeform fabrication. Biomaterials 2003;24:148797. [PubMed: 12527290] 
[191]. Liu CZ, Xia ZD, Han ZW, Hulley PA, Triffitt JT, Czernuszka JT. Novel 3D collagen scaffolds fabricated by indirect printing technique for tissue engineering. J Biomed Mater Res B Appl Biomater 2008;85:519-28. [PubMed: 18076093]

[192]. Silva SS, Mano JF, Reis RL. Potential applications of natural origin polymer-based systems in soft tissue regeneration. Crit Rev Biotechnol 2010;30:200-21. [PubMed: 20735324]

[193]. Liu X, Ma PX. Phase separation, pore structure, and properties of nanofibrous gelatin scaffolds. Biomaterials 2009;30:4094-103. [PubMed: 19481080]

[194]. Djagny VB, Wang Z, Xu S. Gelatin: a valuable protein for food and pharmaceutical industries: review. Crit Rev Food Sci Nutr 2001;41:481-92. [PubMed: 11592686]

[195]. Wang X, Yan Y, Pan Y, Xiong Z, Liu H, Cheng J, et al. Generation of three-dimensional hepatocyte/gelatin structures with rapid prototyping system. Tissue Eng 2006;12:83-90. [PubMed: 16499445]

[196]. Gauvin R, Chen Y-C, Lee JW, Soman P, Zorlutuna P, Nichol JW, et al. Microfabrication of complex porous tissue engineering scaffolds using 3D projection stereolithography. Biomaterials 2012;33:3824-34. [PubMed: 22365811]

[197]. Billiet T, Gevaert E, De Schryver T, Cornelissen M, Dubruel P. The 3D printing of gelatin methacrylamide cell-laden tissue-engineered constructs with high cell viability. Biomaterials 2014;35:49-62. [PubMed: 24112804]

[198]. Lam CXF, Mo XM, Teoh SH, Hutmacher DW. Scaffold development using 3D printing with a starch-based polymer. Mater Sci Eng C 2002;20:49-56.

[199]. Salmoria GV, Klauss P, Paggi RA, Kanis LA, Lago A. Structure and mechanical properties of cellulose based scaffolds fabricated by selective laser sintering. Polym Test 2009;28:648-52.

[200]. Liu I-H, Chang S-H, Lin H-Y. Chitosan-based hydrogel tissue scaffolds made by 3D plotting promotes osteoblast proliferation and mineralization. Biomed Mater Bristol Engl 2015;10:035004.

[201]. Almeida CR, Serra T, Oliveira MI, Planell JA, Barbosa MA, Navarro M. Impact of 3-D printed PLA- and chitosan-based scaffolds on human monocyte/macrophage responses: unraveling the effect of 3-D structures on inflammation. Acta Biomater 2014;10:613-22. [PubMed: 24211731]

[202]. Yan Y, Wang X, Pan Y, Liu H, Cheng J, Xiong Z, et al. Fabrication of viable tissue-engineered constructs with 3D cell-assembly technique. Biomaterials 2005;26:5864-71. [PubMed: 15949552]

[203]. Xu M, Wang X, Yan Y, Yao R, Ge Y. An cell-assembly derived physiological 3D model of the metabolic syndrome, based on adipose-derived stromal cells and a gelatin/alginate/fibrinogen matrix. Biomaterials 2010;31:3868-77. [PubMed: 20153520]

[204]. Xue W, Bandyopadhyay A, Bose S. Polycaprolactone coated porous tricalcium phosphate scaffolds for controlled release of protein for tissue engineering. J Biomed Mater Res B Appl Biomater 2009;91:831-8. [PubMed: 19572301]

[205]. Gunatillake PA, Adhikari R. Biodegradable synthetic polymers for tissue engineering. Eur Cell Mater 2003;5:1-16. discussion 16. [PubMed: 14562275]

[206]. Hutmacher DW, Schantz T, Zein I, Ng KW, Teoh SH, Tan KC. Mechanical properties and cell cultural response of polycaprolactone scaffolds designed and fabricated via fused deposition modeling. J Biomed Mater Res 2001;55:203-16. [PubMed: 11255172]

[207]. Cao T, Ho K-H, Teoh S-H. Scaffold design and in vitro study of osteochondral coculture in a three-dimensional porous polycaprolactone scaffold fabricated by fused deposition modeling. Tissue Eng 2003;9(Suppl 1):S103-12. [PubMed: 14511474]

[208]. Seyednejad H, Gawlitta D, Kuiper RV, de Bruin A, van Nostrum CF, Vermonden T, et al. In vivo biocompatibility and biodegradation of 3D-printed porous scaffolds based on a hydroxylfunctionalized poly( $\varepsilon$-caprolactone). Biomaterials 2012;33:4309-18. [PubMed: 22436798]

[209]. Williams JM, Adewunmi A, Schek RM, Flanagan CL, Krebsbach PH, Feinberg SE, et al. Bone tissue engineering using polycaprolactone scaffolds fabricated via selective laser sintering. Biomaterials 2005;26:4817-27. [PubMed: 15763261]

[210]. Kang H-W, Rhie J-W, Cho D-W. Development of a bi-pore scaffold using indirect solid freeform fabrication based on microstereolithography technology. Microelectron Eng 2009;86:941-4. 
[211]. Kanczler JM, Mirmalek-Sani S-H, Hanley NA, Ivanov AL, Barry JJA, Upton C, et al. Biocompatibility and osteogenic potential of human fetal femur-derived cells on surface selective laser sintered scaffolds. Acta Biomater 2009;5:2063-71. [PubMed: 19362063]

[212]. Antonov EN, Bagratashvili VN, Whitaker MJ, Barry JJA, Shakesheff KM, Konovalov AN, et al. Three-dimensional bioactive and biodegradable scaffolds fabricated by surface-selective laser sintering. Adv Mater 2005;17:327-30.

[213]. Serra T, Planell JA, Navarro M. High-resolution PLA-based composite scaffolds via 3-D printing technology. Acta Biomater 2013;9:5521-30. [PubMed: 23142224]

[214]. Lee M, Dunn JCY, Wu BM. Scaffold fabrication by indirect three-dimensional printing. Biomaterials 2005;26:4281-9. [PubMed: 15683652]

[215]. Kim JY, Park EK, Kim S-Y, Shin J-W, Cho D-W. Fabrication of a SFF-based three-dimensional scaffold using a precision deposition system in tissue engineering. J Micromech Microeng 2008; 18:055027.

[216]. Kim JY, Yoon JJ, Park EK, Kim DS, Kim S-Y, Cho D-W. Cell adhesion and proliferation evaluation of SFF-based biodegradable scaffolds fabricated using a multi-head deposition system. Biofabrication 2009;1:015002. [PubMed: 20811097]

[217]. Lee JW, Lan PX, Kim B, Lim G, Cho D-W. Fabrication and characteristic analysis of a poly(propylene fumarate) scaffold using micro-stereolithography technology. J Biomed Mater Res B Appl Biomater 2008;87B:1-9.

[218]. Lee K-W, Wang S, Fox BC, Ritman EL, Yaszemski MJ, Lu L. Poly(propylene fumarate) bone tissue engineering scaffold fabrication using stereolithography: effects of resin formulations and laser parameters. Biomacromol 2007;8:1077-84.

[219]. Lee JW, Kang KS, Lee SH, Kim J-Y, Lee B-K, Cho D-W. Bone regeneration using a microstereolithography-produced customized poly(propylene fumarate)/diethyl fumarate photopolymer 3D scaffold incorporating BMP-2 loaded PLGA microspheres. Biomaterials 2011;32:744-52. [PubMed: 20933279]

[220]. Kurtz SM, Devine JN. PEEK biomaterials in trauma, orthopedic, and spinal implants. Biomaterials 2007;28:4845-69. [PubMed: 17686513]

[221]. Edwards S, Werkmeister JA, Ramshaw JAM, McLean K, Jarman-Smith M. Polyetheretherketone multifilament and monofilament woven tissue engineering scaffoldsl; 2010.

[222]. Kurtz SM. Chapter 6 - Chemical and radiation stability of PEEK PEEK Biomater. Handb, Oxford: William Andrew Publishing; 2012 p. 75-9.

[223]. Toth JM. Chapter 7 - Biocompatibility of polyaryletheretherketone polymers In: Kurtz SM, editor. PEEK Biomater. Handb, Oxford: William Andrew Publishing; 2012 p. 81-92.

[224]. Vaezi M, Yang S. Extrusion-based additive manufacturing of PEEK for biomedical applications. Virtual Phys Prototyp 2015;10:123-35.

[225]. Jarman-Smith M, Brady M, Kurtz SM, Cordaro NM, Walsh WR. Chapter 12 - Porosity in polyaryletheretherketone PEEK Biomater. Handb, Oxford: William Andrew Publishing; 2012 p. 181-99.

[226]. Zhao Y, Wong HM, Wang W, Li P, Xu Z, Chong EYW, et al. Cytocompatibility, osseointegration, and bioactivity of three-dimensional porous and nanostructured network on polyetheretherketone. Biomaterials 2013;34:9264-77. [PubMed: 24041423]

[227]. Wang L, He S, Wu X, Liang S, Mu Z, Wei J, et al. Polyetheretherketone/nanofluorohydroxyapatite composite with antimicrobial activity and osseointegration properties. Biomaterials 2014;35:6758-75. [PubMed: 24835045]

[228]. Tan KH, Chua CK, Leong KF, Cheah CM, Cheang P, Abu Bakar MS, et al. Scaffold development using selective laser sintering of polyetheretherketone-hydroxyapatite biocomposite blends. Biomaterials 2003;24:3115-23. [PubMed: 12895584]

[229]. von Wilmowsky C, Vairaktaris E, Pohle D, Rechtenwald T, Lutz R, Münstedt H, et al. Effects of bioactive glass and beta-TCP containing three-dimensional laser sintered polyetheretherketone composites on osteoblasts in vitro. J Biomed Mater Res A 2008;87:896-902. [PubMed: 18228252] 
[230]. Pilliar RM, Filiaggi MJ, Wells JD, Grynpas MD, Kandel RA. Porous calcium polyphosphate scaffolds for bone substitute applications - in vitro characterization. Biomaterials 2001;22:96372. [PubMed: 11311015]

[231]. Omelon S, Baer A, Coyle T, Pilliar RM, Kandel R, Grynpas M. Polymeric crystallization and condensation of calcium polyphosphate glass. Mater Res Bull 2008;43:68-80.

[232]. Wang K, Chen F, Liu C, Rüssel C. The effect of polymeric chain-like structure on the degradation and cellular biocompatibility of calcium polyphosphate. Mater Sci Eng C 2008;28:1572-8.

[233]. Shanjani Y, De Croos JNA, Pilliar RM, Kandel RA, Toyserkani E. Solid freeform fabrication and characterization of porous calcium polyphosphate structures for tissue engineering purposes. J Biomed Mater Res B Appl Biomater 2010;93:510-9. [PubMed: 20162726]

[234]. Shanjani Y, Hu Y, Pilliar RM, Toyserkani E. Mechanical characteristics of solid-freeformfabricated porous calcium polyphosphate structures with oriented stacked layers. Acta Biomater 2011;7:1788-96. [PubMed: 21185409]

[235]. Shanjani Y, Hu Y, Toyserkani E, Grynpas M, Kandel RA, Pilliar RM. Solid freeform fabrication of porous calcium polyphosphate structures for bone substitute applications: in vivo studies. J Biomed Mater Res B Appl Biomater 2013;101B:972-80.

[236]. Kalita SJ, Bose S, Hosick HL, Bandyopadhyay A. Development of controlled porosity polymerceramic composite scaffolds via fused deposition modeling. Mater Sci Eng C 2003;23:611-20.

[237]. Korpela J, Kokkari A, Korhonen H, Malin M, Närhi T, Seppälä J. Biodegradable and bioactive porous scaffold structures prepared using fused deposition modeling. J Biomed Mater Res B Appl Biomater 2013;101B:610-9.

[238]. Fedorovich NE, De Wijn JR, Verbout AJ, Alblas J, Dhert WJA. Three-dimensional fiber deposition of cell-laden, viable, patterned constructs for bone tissue printing. Tissue Eng Part A 2008;14:127-33. [PubMed: 18333811]

[239]. Luo Y, Wu C, Lode A, Gelinsky M. Hierarchical mesoporous bioactive glass/alginate composite scaffolds fabricated by three-dimensional plotting for bone tissue engineering. Biofabrication 2013;5:015005. [PubMed: 23228963]

[240]. Wiria FE, Leong KF, Chua CK, Liu Y. Poly-epsilon-caprolactone/hydroxyapatite for tissue engineering scaffold fabrication via selective laser sintering. Acta Biomater 2007;3:1-12. [PubMed: 17055789]

[241]. Duan B, Wang M, Zhou WY, Cheung WL, Li ZY, Lu WW. Three-dimensional nanocomposite scaffolds fabricated via selective laser sintering for bone tissue engineering. Acta Biomater 2010;6:4495-505. [PubMed: 20601244]

[242]. Lindner M, Hoeges S, Meiners W, Wissenbach K, Smeets R, Telle R, et al. Manufacturing of individual biodegradable bone substitute implants using selective laser melting technique. $\mathrm{J}$ Biomed Mater Res A 2011;97A:466-71.

[243]. Ronca A, Ambrosio L, Grijpma DW. Preparation of designed poly(d, 1-lactide)/nanosized hydroxyapatite composite structures by stereolithography. Acta Biomater 2013;9:5989-96. [PubMed: 23232210]

[244]. Elomaa L, Kokkari A, Närhi T, Seppälä JV. Porous 3D modeled scaffolds of bioactive glass and photocrosslinkable poly( $\varepsilon$-caprolactone) by stereolithography. Compos Sci Technol 2013;74:99106.

[245]. Inzana JA, Olvera D, Fuller SM, Kelly JP, Graeve OA, Schwarz EM, et al. 3D printing of composite calcium phosphate and collagen scaffolds for bone regeneration. Biomaterials 2014;35:4026-34. [PubMed: 24529628]

[246]. Puppi D, Mota C, Gazzarri M, Dinucci D, Gloria A, Myrzabekova M, et al. Additive manufacturing of wet-spun polymeric scaffolds for bone tissue engineering. Biomed Microdevices 2012;14:1115-27. [PubMed: 22767245]

[247]. Eosoly S, Brabazon D, Lohfeld S, Looney L. Selective laser sintering of hydroxyapatite/poly- $\varepsilon-$ caprolactone scaffolds. Acta Biomater 2010;6:2511-7. [PubMed: 19616649]

[248]. Wiria FE. KFL. Poly- $\varepsilon$-caprolactone/hydroxyapatite for tissue engineering scaffold fabrication via selective laser sintering. Acta Biomater 2007;3:1-12. [PubMed: 17055789] 
[249]. Kim JY, Jin G-Z, Park IS, Kim J-N, Chun SY, Park EK, et al. Evaluation of solid free-form fabrication-based scaffolds seeded with osteoblasts and human umbilical vein endothelial cells for use in vivo osteogenesis. Tissue Eng Part A 2010;16:2229-36. [PubMed: 20163199]

[250]. Lam CX, Teoh SH, Hutmacher DW. Comparison of the degradation of polycaprolactone and polycaprolactone-( $\beta$-tricalcium phosphate) scaffolds in alkaline medium. Polym Int 2007;56:718-28.

[251]. Lam CXF, Hutmacher DW, Schantz J-T, Woodruff MA, Teoh SH. Evaluation of polycaprolactone scaffold degradation for 6 months in vitro and in vivo. J Biomed Mater Res A 2009;90:906-19. [PubMed: 18646204]

[252]. Lam CXF, Savalani MM, Teoh S-H, Hutmacher DW. Dynamics of in vitro polymer degradation of polycaprolactone-based scaffolds: accelerated versus simulated physiological conditions. Biomed Mater Bristol Engl 2008;3:034108.

[253]. Xiong Z, Yan Y, Wang S, Zhang R, Zhang C. Fabrication of porous scaffolds for bone tissue engineering via low-temperature deposition. Scr Mater 2002;46:771-6.

[254]. Sánchez-Salcedo S, Nieto A, Vallet-Regí M. Hydroxyapatite/ $\beta$-tricalcium phosphate/agarose macroporous scaffolds for bone tissue engineering. Chem Eng J 2008;137:62-71.

[255]. Mondrinos MJ, Dembzynski R, Lu L, Byrapogu VKC, Wootton DM, Lelkes PI, et al. Porogenbased solid freeform fabrication of polycaprolactone-calcium phosphate scaffolds for tissue engineering. Biomaterials 2006;27:4399-408. [PubMed: 16678255]

[256]. Schwartz I, Robinson BP, Hollinger JO, Szachowicz EH, Brekke J. Calvarial bone repair with porous D, L-polylactide. Otolaryngol Head Neck Surg 1995;112:707-13. [PubMed: 7777356]

[257]. Boyan BD, Hummert TW, Dean DD, Schwartz Z. Role of material surfaces in regulating bone and cartilage cell response. Biomaterials 1996;17:137-46. [PubMed: 8624390]

[258]. Leong KF, Cheah CM, Chua CK. Solid freeform fabrication of three-dimensional scaffolds for engineering replacement tissues and organs. Biomaterials 2003;24:2363-78. [PubMed: 12699674]

[259]. Nigam SK, Wu W, Bush KT. Organogenesis forum lecture: in vitro kidney development, tissue engineering and systems biology. Organogenesis 2008;4:137-43. [PubMed: 19279725]

[260]. Guillotin B, Guillemot F. Cell patterning technologies for organotypic tissue fabrication. Trends Biotechnol 2011;29:183-90. [PubMed: 21256609]

[261]. Malda J, Visser J, Melchels FP, Jüngst T, Hennink WE, Dhert WJA, et al. 25th Anniversary article: engineering hydrogels for biofabrication. Adv Mater 2013;25:5011-28. [PubMed: 24038336]

[262]. Murphy SV, Atala A. 3D bioprinting of tissues and organs. Nat Biotechnol 2014;32:773-85. [PubMed: 25093879]

[263]. Fierz FC, Beckmann F, Huser M, Irsen SH, Leukers B, Witte F, et al. The morphology of anisotropic 3D-printed hydroxyapatite scaffolds. Biomaterials 2008;29:3799-806. [PubMed: 18606446]

[264]. Fielding G, Bose $\mathrm{S} . \mathrm{SiO}_{2}$ and $\mathrm{ZnO}$ dopants in three-dimensionally printed tricalcium phosphate bone tissue engineering scaffolds enhance osteogenesis and angiogenesis in vivo. Acta Biomater 2013;9:9137-48. [PubMed: 23871941]

[265]. Gao G, Schilling AF, Yonezawa T, Wang J, Dai G, Cui X. Bioactive nanoparticles stimulate bone tissue formation in bioprinted three-dimensional scaffold and human mesenchymal stem cells. Biotechnol J 2014;9:1304-11. [PubMed: 25130390]

[266]. Ker EDF, Nain AS, Weiss LE, Wang J, Suhan J, Amon CH, et al. Bioprinting of growth factors onto aligned sub-micron fibrous scaffolds for simultaneous control of cell differentiation and alignment. Biomaterials 2011;32:8097-107. [PubMed: 21820736]

[267]. Gagg G, Ghassemieh E, Wiria FE. Effects of sintering temperature on morphology and mechanical characteristics of $3 \mathrm{D}$ printed porous titanium used as dental implant. Mater Sci Eng C 2013;33:3858-64.

[268]. Kim K-B, Kim W-C, Kim H-Y, Kim J-H. An evaluation of marginal fit of three-unit fixed dental prostheses fabricated by direct metal laser sintering system. Dent Mater 2013;29:e91-6. [PubMed: 23680302] 
[269]. Tamimi F, Torres J, Al-Abedalla K, Lopez-Cabarcos E, Alkhraisat MH, Bassett DC, et al. Osseointegration of dental implants in 3D-printed synthetic onlay grafts customized according to bone metabolic activity in recipient site. Biomaterials 2014;35:5436-45. [PubMed: 24726538]

[270]. Wang J, Shaw LL, Cameron TB. Solid freeform fabrication of permanent dental restorations via slurry micro-extrusion. J Am Ceram Soc 2006;89:346-9.

[271]. Sherwood JK, Riley SL, Palazzolo R, Brown SC, Monkhouse DC, Coates M, et al. A threedimensional osteochondral composite scaffold for articular cartilage repair. Biomaterials 2002;23:4739-51. [PubMed: 12361612]

[272]. Woodfield TBF, Malda J, de Wijn J, Péters F, Riesle J, van Blitterswijk CA. Design of porous scaffolds for cartilage tissue engineering using a three-dimensional fiber-deposition technique. Biomaterials 2004;25:4149-61. [PubMed: 15046905]

[273]. Cui X, Breitenkamp K, Finn MG, Lotz M, D'Lima DD. Direct human cartilage repair using three-dimensional bioprinting technology. Tissue Eng Part A 2012;18:1304-12. [PubMed: 22394017]

[274]. Fedorovich NE, Schuurman W, Wijnberg HM, Prins H-J, van Weeren PR, Malda J, et al. Biofabrication of osteochondral tissue equivalents by printing topologically defined, cell-laden hydrogel scaffolds. Tissue Eng Part C Methods 2012;18:33-44. [PubMed: 21854293]

[275]. Gurkan UA, El Assal R, Yildiz SE, Sung Y, Trachtenberg AJ, Kuo WP, et al. Engineering anisotropic biomimetic fibrocartilage microenvironment by bioprinting mesenchymal stem cells in nanoliter gel droplets. Mol Pharm 2014;11:2151-9. [PubMed: 24495169]

[276]. Hung K-C, Tseng C-S, Hsu S-H. Synthesis and 3D printing of biodegradable polyurethane elastomer by a water-based process for cartilage tissue engineering applications. Adv Healthc Mater 2014;3:1578-87. [PubMed: 24729580]

[277]. Park JY, Choi J-C, Shim J-H, Lee J-S, Park H, Kim SW, et al. A comparative study on collagen type I and hyaluronic acid dependent cell behavior for osteochondral tissue bioprinting. Biofabrication 2014;6:035004. [PubMed: 24758832]

[278]. Shim J-H, Lee J-S, Kim JY, Cho D-W. Bioprinting of a mechanically enhanced threedimensional dual cell-laden construct for osteochondral tissue engineering using a multi-head tissue/organ building system. J Micromech Microeng 2012;22:085014.

[279]. Xu T, Binder KW, Albanna MZ, Dice D, Zhao W, Yoo JJ, et al. Hybrid printing of mechanically and biologically improved constructs for cartilage tissue engineering applications. Biofabrication 2013;5:015001. [PubMed: 23172542]

[280]. Park CH, Rios HF, Jin Q, Bland ME, Flanagan CL, Hollister SJ, et al. Biomimetic hybrid scaffolds for engineering human tooth-ligament interfaces. Biomaterials 2010;31:5945-52. [PubMed: 20471083]

[281]. Park CH, Rios HF, Jin Q, Sugai JV, Padial-Molina M, Taut AD, et al. Tissue engineering boneligament complexes using fiber-guiding scaffolds. Biomaterials 2012;33:137-45. [PubMed: 21993234]

[282]. Merceron TK, Burt M, Seol Y-J, Kang H-W, Lee SJ, Yoo JJ, et al. A 3D bioprinted complex structure for engineering the muscle-tendon unit. Biofabrication 2015;7:035003. [PubMed: 26081669]

[283]. Hockaday LA, Kang KH, Colangelo NW, Cheung PYC, Duan B, Malone E, et al. Rapid 3D printing of anatomically accurate and mechanically heterogeneous aortic valve hydrogel scaffolds. Biofabrication 2012;4:035005. [PubMed: 22914604]

[284]. Cui X, Boland T. Human microvasculature fabrication using thermal inkjet printing technology. Biomaterials 2009;30:6221-7. [PubMed: 19695697]

[285]. Amal Owida, Rui Chen, Shital Patel, Yos Morsi, Xiumei Mo. Artery vessel fabrication using the combined fused deposition modeling and electrospinning techniquesnull. Rapid Prototyp J 2011;17:37-44.

[286]. Skardal A, Zhang J, Prestwich GD. Bioprinting vessel-like constructs using hyaluronan hydrogels crosslinked with tetrahedral polyethylene glycol tetracrylates. Biomaterials 2010;31:6173-81. [PubMed: 20546891] 
[287]. Zhao L, Lee VK, Yoo S-S, Dai G, Intes X. The integration of 3-D cell printing and mesoscopic fluorescence molecular tomography of vascular constructs within thick hydrogel scaffolds. Biomaterials 2012;33:5325-32. [PubMed: 22531221]

[288]. Lee W, Debasitis JC, Lee VK, Lee J-H, Fischer K, Edminster K, et al. Multi-layered culture of human skin fibroblasts and keratinocytes through three-dimensional freeform fabrication. Biomaterials 2009;30:1587-95. [PubMed: 19108884]

[289]. Koch L, Kuhn S, Sorg H, Gruene M, Schlie S, Gaebel R, et al. Laser printing of skin cells and human stem cells. Tissue Eng Part C Methods 2010;16:847-54. [PubMed: 19883209]

[290]. Koch L, Deiwick A, Schlie S, Michael S, Gruene M, Coger V, et al. Skin tissue generation by laser cell printing. Biotechnol Bioeng 2012;109:1855-63. [PubMed: 22328297]

[291]. Michael S, Sorg H, Peck C-T, Koch L, Deiwick A, Chichkov B, et al. Tissue engineered skin substitutes created by laser-assisted bioprinting form skin-like structures in the dorsal skin fold chamber in mice. PLoS ONE 2013;8:e57741. [PubMed: 23469227]

[292]. Markstedt K, Mantas A, Tournier I, Martínez Ávila H, Hägg D, Gatenholm P. 3D bioprinting human chondrocytes with nanocellulose-alginate bioink for cartilage tissue engineering applications. Biomacromol 2015;16:1489-96.

[293]. Lee J-S, Hong JM, Jung JW, Shim J-H, Oh J-H, Cho D-W. 3D printing of composite tissue with complex shape applied to ear regeneration. Biofabrication 2014;6:024103. [PubMed: 24464765]

[294]. Mannoor MS, Jiang Z, James T, Kong YL, Malatesta KA, Soboyejo WO, et al. 3D printed bionic ears. Nano Lett 2013;13:2634-9. [PubMed: 23635097]

[295]. Jiankang H, Dichen L, Yaxiong L, Bo Y, Hanxiang Z, Qin L, et al. Preparation of chitosangelatin hybrid scaffolds with well-organized microstructures for hepatic tissue engineering. Acta Biomater 2009;5:453-61. [PubMed: 18675601]

[296]. Huang H, Oizumi S, Kojima N, Niino T, Sakai Y. Avidin-biotin binding-based cell seeding and perfusion culture of liver-derived cells in a porous scaffold with a three-dimensional interconnected flow-channel network. Biomaterials 2007;28:3815-23. [PubMed: 17544499]

[297]. Zein NN, Hanouneh IA, Bishop PD, Samaan M, Eghtesad B, Quintini C, et al. Threedimensional print of a liver for preoperative planning in living donor liver transplantation. Liver Transplant Off Publ Am Assoc Study Liver Dis Int Liver Transplant Soc 2013;19:1304-10.

[298]. Chou D-T, Wells D, Hong D, Lee B, Kuhn H, Kumta PN. Novel processing of iron-manganese alloy-based biomaterials by inkjet 3-D printing. Acta Biomater 2013;9:8593-603. [PubMed: 23624222]

[299]. Bandyopadhyay A, Bose S. Bone replacement materials. US20070203584 A1; 2007.

[300]. Sahasrabudhe H, Bose S, Bandyopadhyay A. Laser processed calcium phosphate reinforced CoCrMo for load-bearing applications: Processing and wear induced damage evaluation. Acta Biomaterialia 2017. doi: 10.1016/j.actbio.2017.11.022.

[301]. Liu F, Leslie I, Williams S, Fisher J, Jin Z. Development of computational wear simulation of metal-on-metal hip resurfacing replacements. J Biomech 2008;41(3):686-94. [PubMed: 17976628]

[302]. Lord JK, Langton DJ, Nargol AVF, Joyce TJ. Volumetric wear assessment of failed metal-onmetal hip resurfacing prostheses. Wear 2011;272(1):79-87.

[303]. Kim J, McBride S, Tellis B, Alvarez-Urena P, Song Y-H, Dean DD, et al. Rapid-prototyped PLGA/ $\beta$-TCP/hydroxyapatite nanocomposite scaffolds in a rabbit femoral defect model. Biofabrication 2012;4:025003. [PubMed: 22427485]

[304]. Gutiérrez-Salazar Mdel P, Reyes-Gasga J. Microhardness and chemical composition of human tooth. Mater Res 2003;6:367-73.

[305]. Kasparova M, Grafova L, Dvorak P, Dostalova T, Prochazka A, Eliasova H, et al. Possibility of reconstruction of dental plaster cast from 3D digital study models. Biomed Eng OnLine 2013;12:1-11. [PubMed: 23289769]

[306]. Hazeveld A, Huddleston Slater JJR, Ren Y. Accuracy and reproducibility of dental replica models reconstructed by different rapid prototyping techniques. Am J Orthod Dentofacial Orthop 2014;145:108-15. [PubMed: 24373661] 
[307]. Sahoo S, Ang L-T, Cho-Hong Goh J, Toh S-L. Bioactive nanofibers for fibroblastic differentiation of mesenchymal precursor cells for ligament/tendon tissue engineering applications. Differentiation 2010;79:102-10. [PubMed: 19963313] 


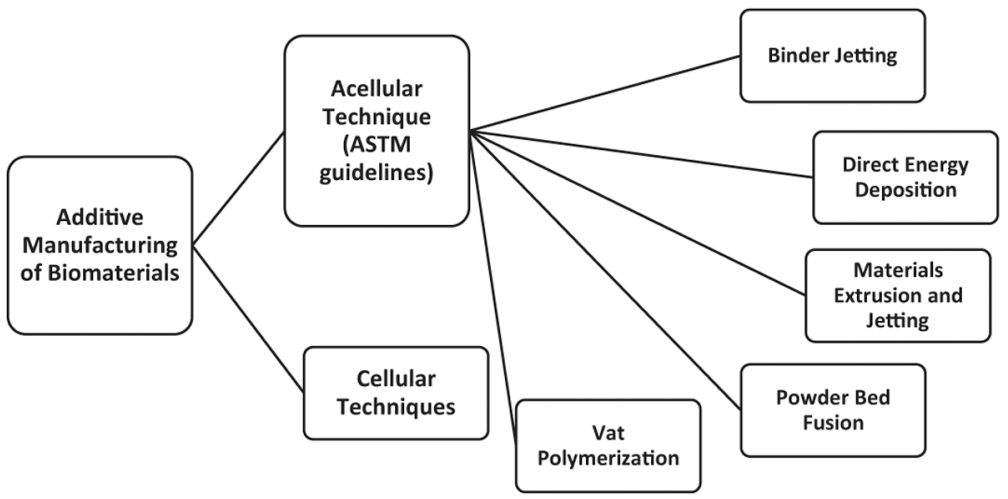

Fig. 1.

Acellular techniques for the additive manufacturing of biomaterials. 

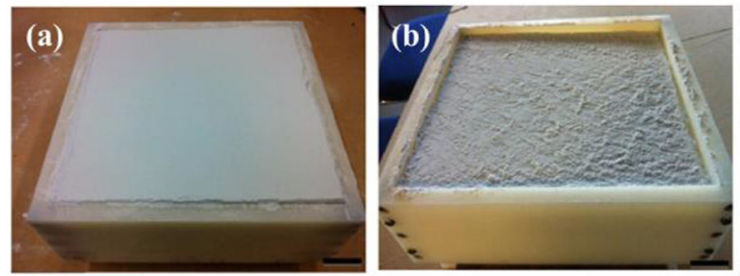

Powder properties for starting $\mathrm{CaP}$ and $\mathrm{CaSO}_{4}$ materials.

\begin{tabular}{|c|c|c|c|c|c|}
\hline \multirow[t]{2}{*}{ Powder Properties } & \multirow[t]{2}{*}{$\mathrm{CaSO}_{4}$} & \multicolumn{2}{|l|}{$\mathrm{HA}$} & \multicolumn{2}{|l|}{$\beta$-TCP } \\
\hline & & Coarse & Fine & Coarse & Fine \\
\hline$D_{10}(\mu \mathrm{m})$ & 28.12 & 45.68 & 1.02 & 32.63 & 6.22 \\
\hline $\mathrm{D}_{50}(\mu \mathrm{m})$ & 67.84 & 65.35 & 2.33 & 54.50 & 13.04 \\
\hline$D_{s o}(\mu \mathrm{m})$ & 101.83 & 94.40 & 3.49 & 109.29 & 19.92 \\
\hline True Particle Density & 2.760 & 3.156 & 3.156 & 3.140 & 3.140 \\
\hline
\end{tabular}

Powder properties

$\mathrm{HA}$ (coarse): $\mathrm{CaSO}_{4}$

$\rho\left(\mathrm{g} \mathrm{cm}^{-3}\right)$

\begin{tabular}{|c|c|c|c|c|}
\hline \multirow[t]{2}{*}{ Powder properties } & \multicolumn{2}{|c|}{$\mathrm{HA}$ (coarse): $\mathrm{CaSO}_{4}$} & \multicolumn{2}{|l|}{$\mathrm{HA}$ (fine): $\mathrm{CaSO}_{4}$} \\
\hline & $25: 75$ wt.\% & $50: 50$ wt.\% & $25: 75$ wt.\% & $50: 50$ wt.\% \\
\hline True particle density $\left(\mathrm{g} \mathrm{cm}^{-3}\right)$ & 2.859 & 2.958 & 2.859 & 2.958 \\
\hline Bulk density $\left(\mathrm{g} \mathrm{cm}^{-3}\right)$ & $1.070 \pm 0.008$ & $1.160 \pm 0.010$ & $0.802 \pm 0.008$ & $0.618 \pm 0.004$ \\
\hline In-process powder bed density $\left(\mathrm{g} \mathrm{cm}^{-3}\right)$ & $1.079 \pm 0.040$ & $1.131 \pm 0.034$ & $0.808 \pm 0.056$ & $0.752 \pm 0.035$ \\
\hline Powder bed packing ratio & $38 \% \pm 1 \%$ & $38 \% \pm 1 \%$ & $28 \% \pm 2 \%$ & $25 \% \pm 1 \%$ \\
\hline \multirow[t]{2}{*}{ Powder Properties } & \multicolumn{2}{|c|}{$\beta$-TCP (coarse): $\mathrm{CaSO}_{4}$} & \multicolumn{2}{|c|}{$\beta-\mathrm{TCP}$ (fine): $\mathrm{CaSO}_{4}$} \\
\hline & $25: 75$ wt.\% & 50:50 wt.\% & $25: 75$ wt. $\%$ & $50: 50$ wt\% \\
\hline True Partide Density $\left(\mathrm{g} \mathrm{cm}^{-3}\right)$ & 2.855 & 2.950 & 2.855 & 2.950 \\
\hline Bulk Density $\left(\mathrm{g} \mathrm{cm}^{-3}\right)$ & $0.823 \pm 0.006$ & $0.763 \pm 0.006$ & $0.640 \pm 0.009$ & $0.531 \pm 0.005$ \\
\hline In-process powder bed density $\left(\mathrm{g} \mathrm{cm}^{-3}\right)$ & $0.923 \pm 0.035$ & $0.898 \pm 0.054$ & $0.620 \pm 0.063$ & $0.552 \pm 0.050$ \\
\hline Powder bed packing ratio & $32 \% \pm 1 \%$ & $30 \% \pm 2 \%$ & $22 \% \pm 2 \%$ & $19 \% \pm 2 \%$ \\
\hline
\end{tabular}

Fig. 2.

Image (a) and (b) showed low building surface roughness using $\mathrm{CaP}$ (coarse): $\mathrm{CaSO}_{4}$ and high building surface roughness using $\mathrm{CaP}$ (fine): $\mathrm{CaSO}_{4}$. Three tables were the starting powder parameters, densities and packing ratios for different $\mathrm{CaP}(\beta-\mathrm{TCP}$ and $\mathrm{HA}) / \mathrm{CaSO}_{4}$ system [11]. 


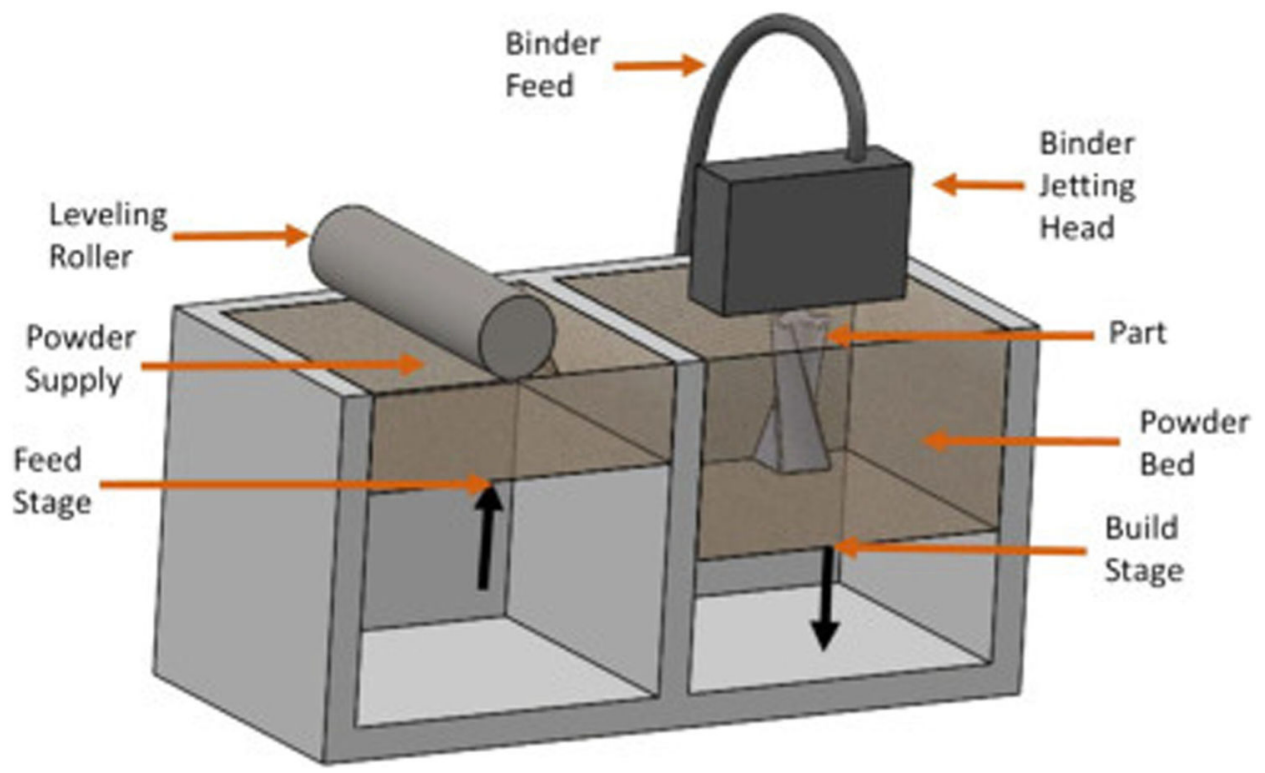

Fig. 3.

The schematic of the binder jetting machine [41]. 


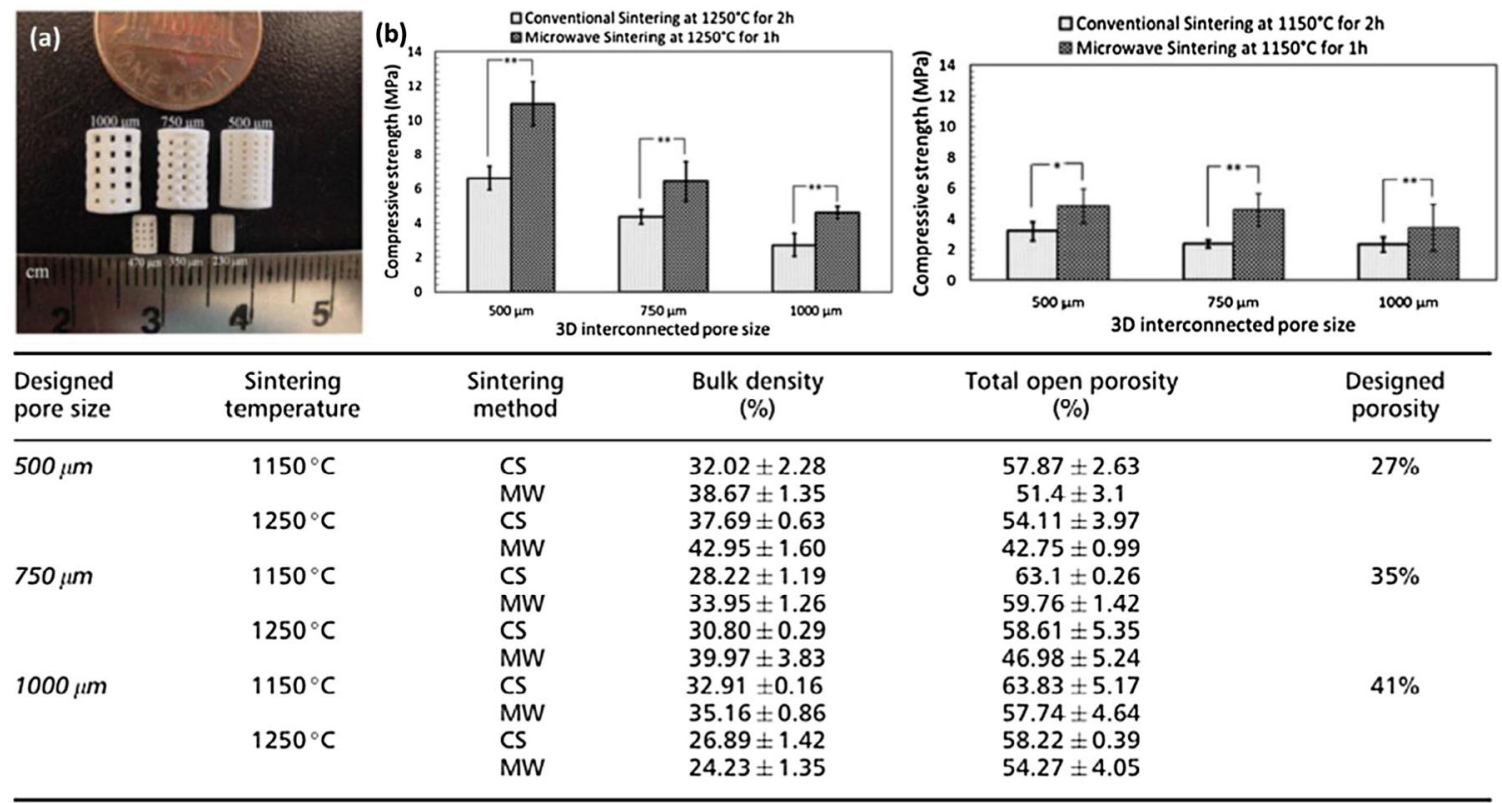

Note: CS: conventional sintering, MW: microwave sintering.

Fig. 4.

The comparison of density and compressive strength between conventional and microwave sintering. The printed parts were shown in (a). (b) was the compressive strength result for different pore size of samples and different sintering conditions. The table included the density and porosity data for different pore size of samples and different sintering conditions [47]. 


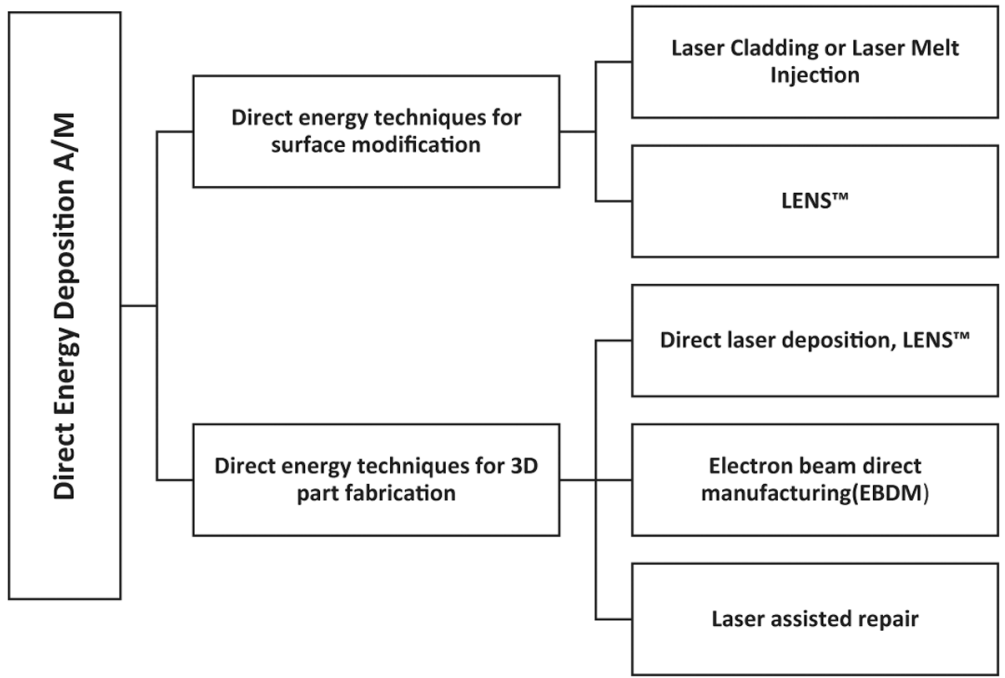

Fig. 5.

Classification of direct energy deposition techniques of additive manufacturing. 


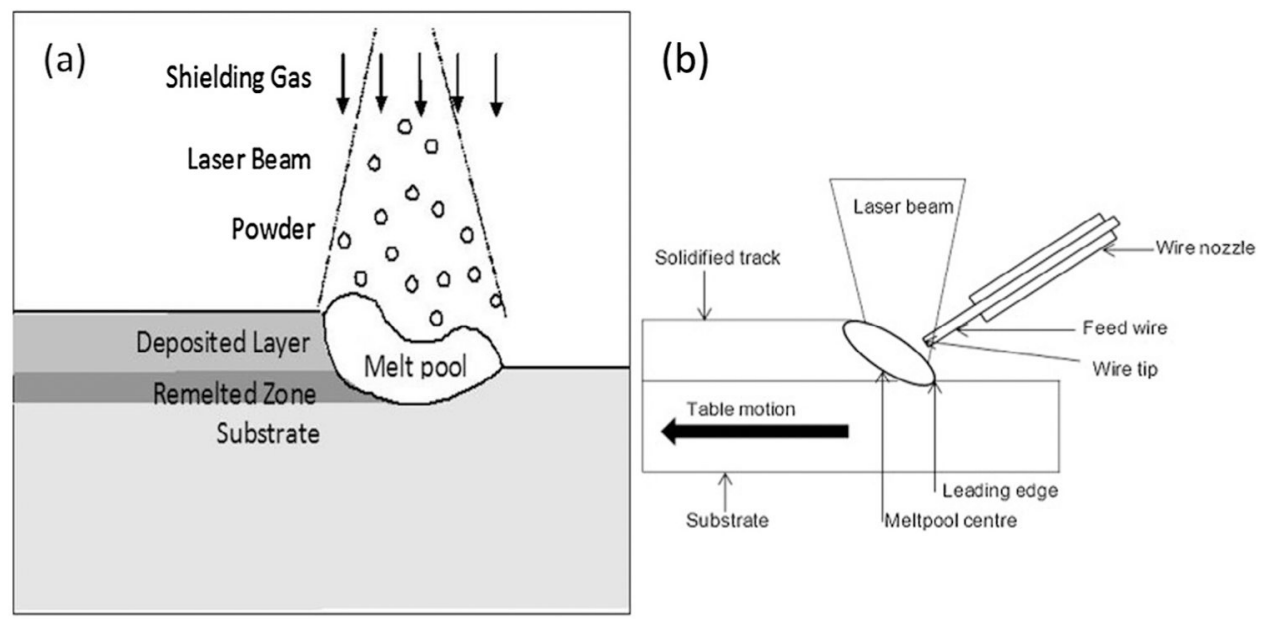

Fig. 6.

(a) Laser melt injection process using powder [52] and (b) Schematic of laser cladding process using wire or filament feed. 


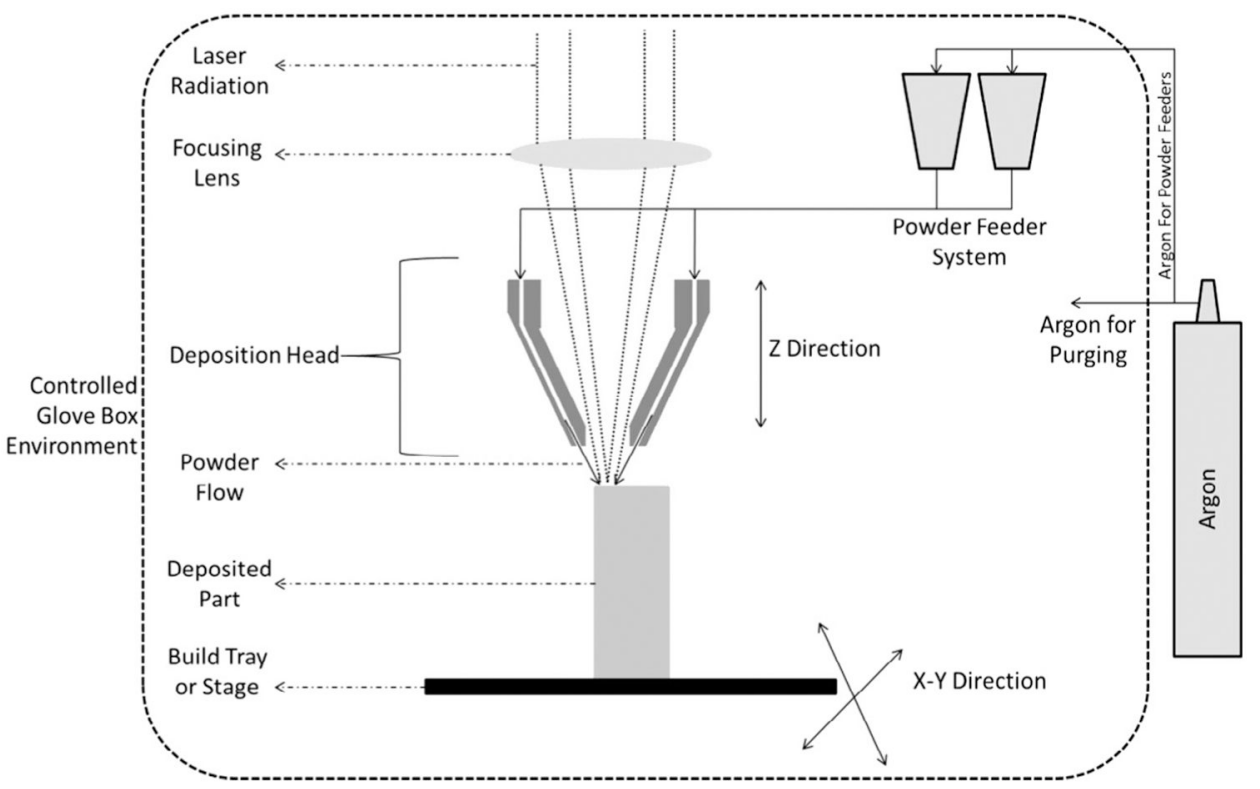

Fig. 7.

Schematic representation of Laser Engineered Net Shaping (LENS ${ }^{\mathrm{TM}}$ ) process [55]. 


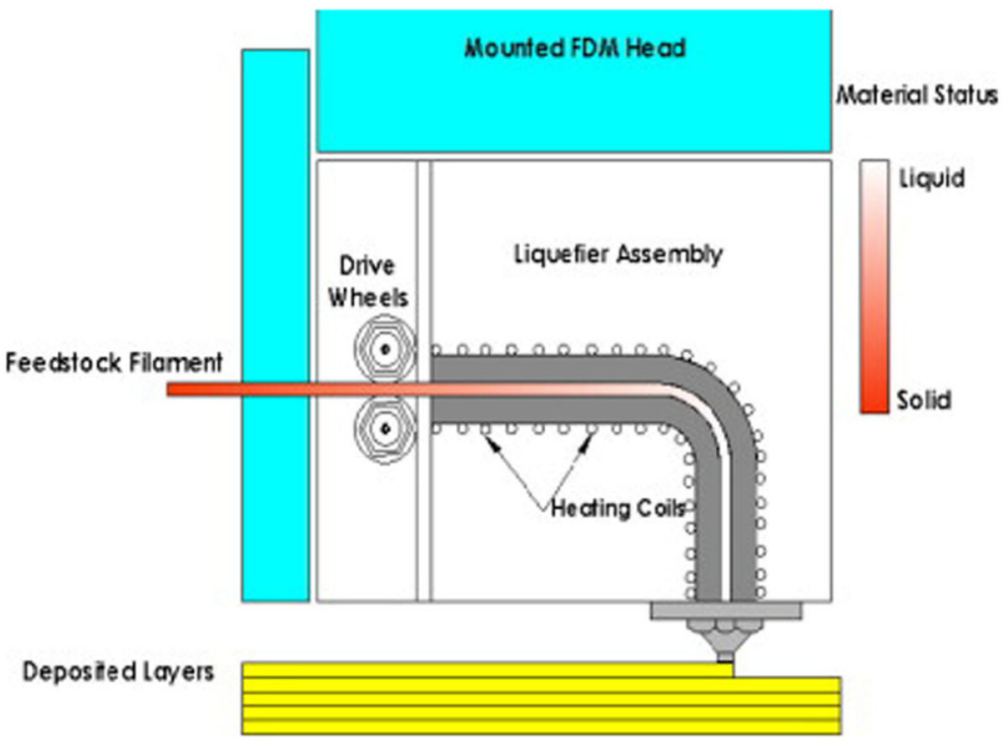

Fig. 8.

Fused deposition modeling process [67]. 


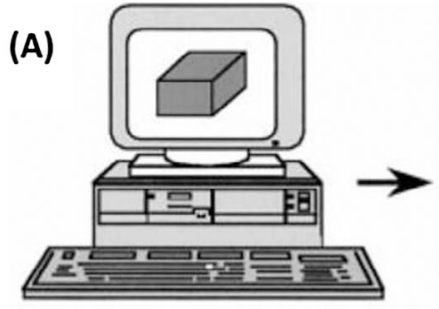

CAD File
(B)

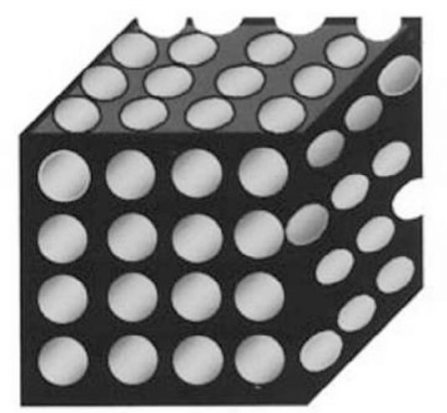

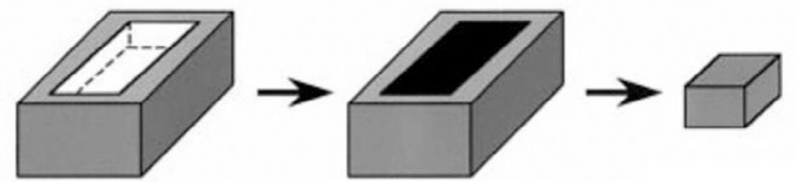

Polymer Mold
Slurry Infiltration
Sintered Structure

(C)

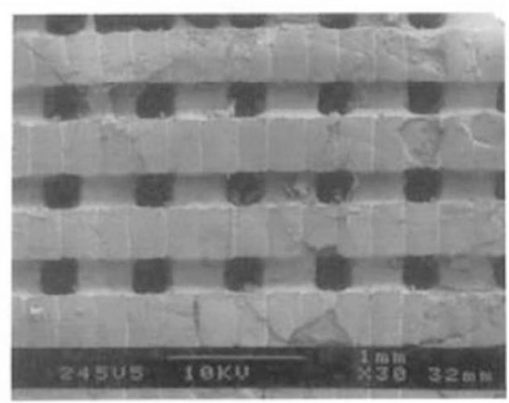

Fig. 9.

(A) Schematic of the indirect FDM processing for making porous ceramic structure. (B) and (C) are schematic and microstructure of the sintered porous ceramic part [69]. 


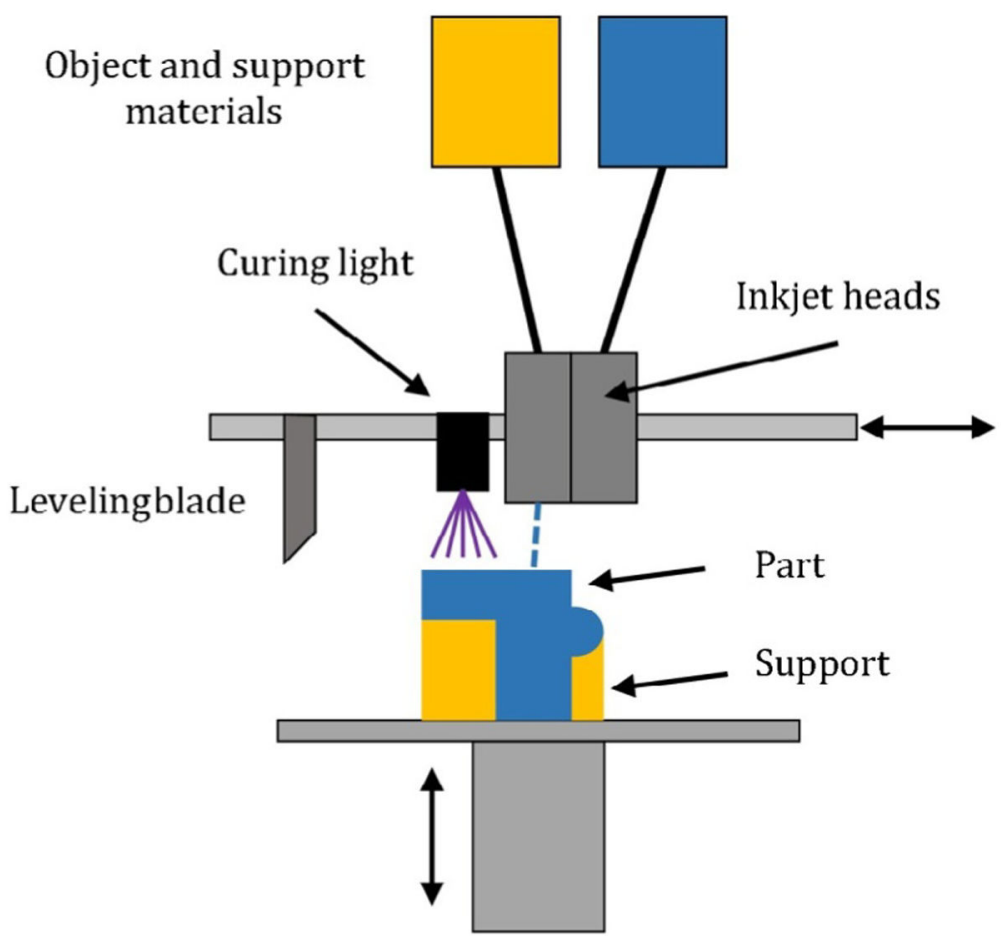

Fig. 10.

Schematic Representation of Material Jetting (MultiJet or PolyJet) Process [70]. 


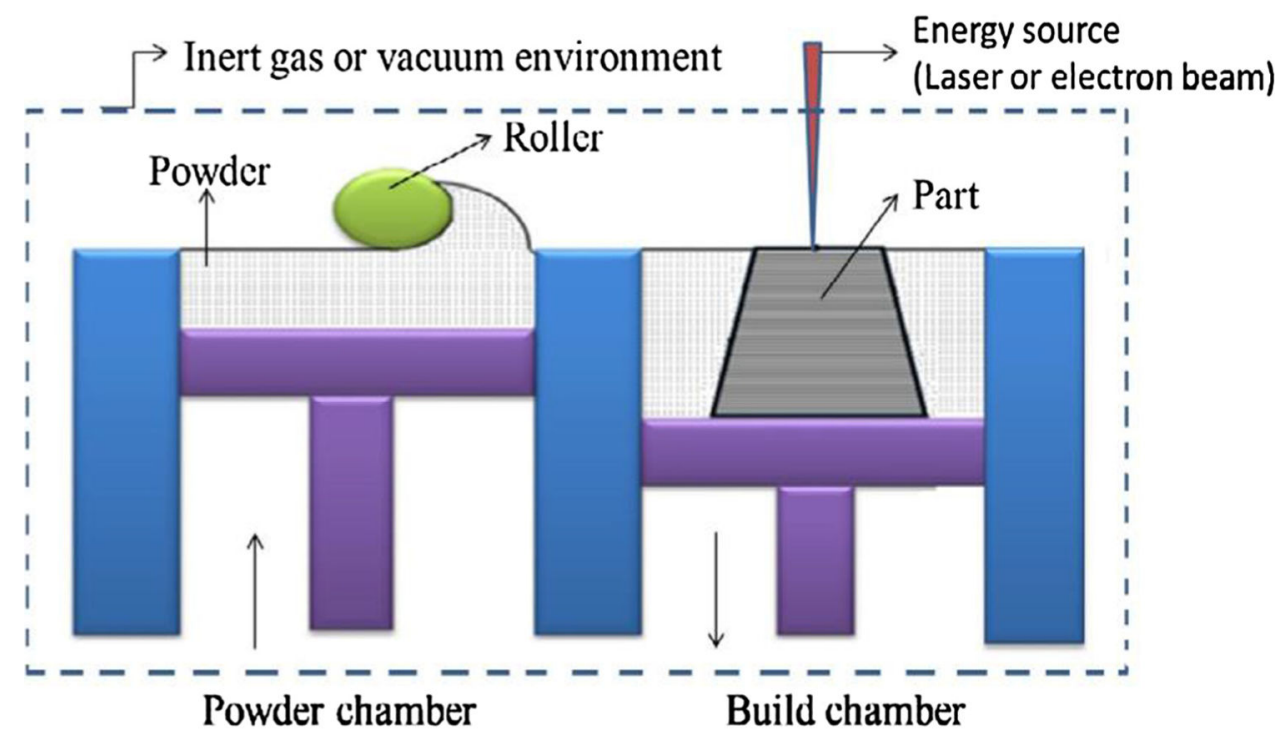

Fig. 11.

Schematic of the powder fusion process [74]. 


\begin{tabular}{|c|c|}
\hline$\prod_{0}^{O}{ }^{O} R$ & ${ }_{n} \underset{O}{>} R$ \\
\hline Photoinitiator & $\downarrow$ \\
\hline$\left.+\mathrm{C}^{\mathrm{H}}-\underset{\mathrm{H}}{\mathrm{C}}\right)_{n}^{\mathrm{O}}$ & $\left(-\mathrm{O}-\mathrm{H}_{2} \stackrel{\mathrm{R}}{\mathrm{I}} \underset{\mathrm{H}}{\mathrm{H}}\right)_{\mathrm{n}}$ \\
\hline Free-radical polymerization & Cationic polymerization \\
\hline
\end{tabular}

Fig. 12.

Chemical equations of free-radical polymerization and cationic polymerization for vat polymerization process. 
a

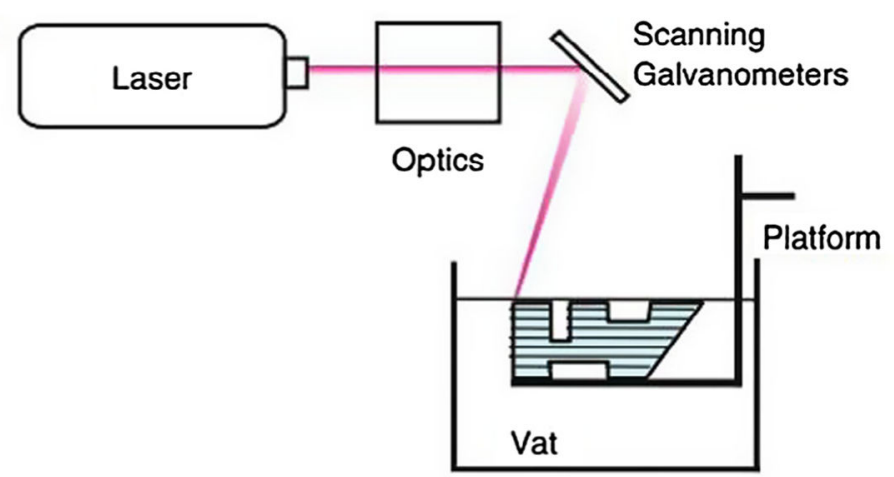

schematic of vector scan

b

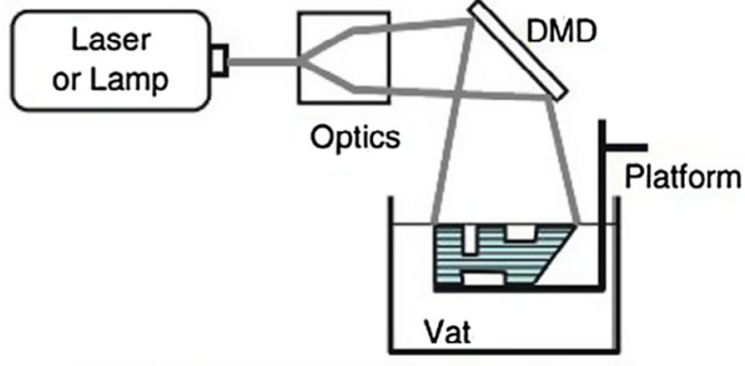

Schematic of mask projection approach c

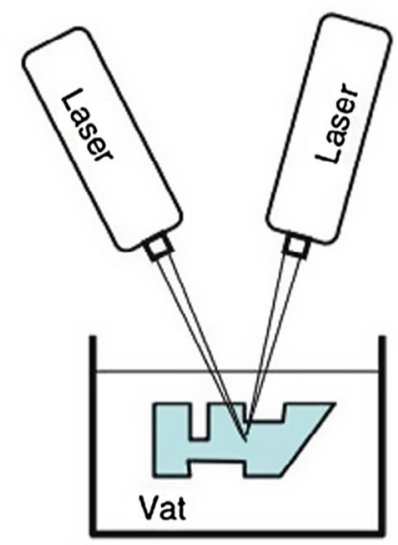

Two-photon approach

Fig. 13.

Schematics of three different light sources for vat polymerization [71]. 

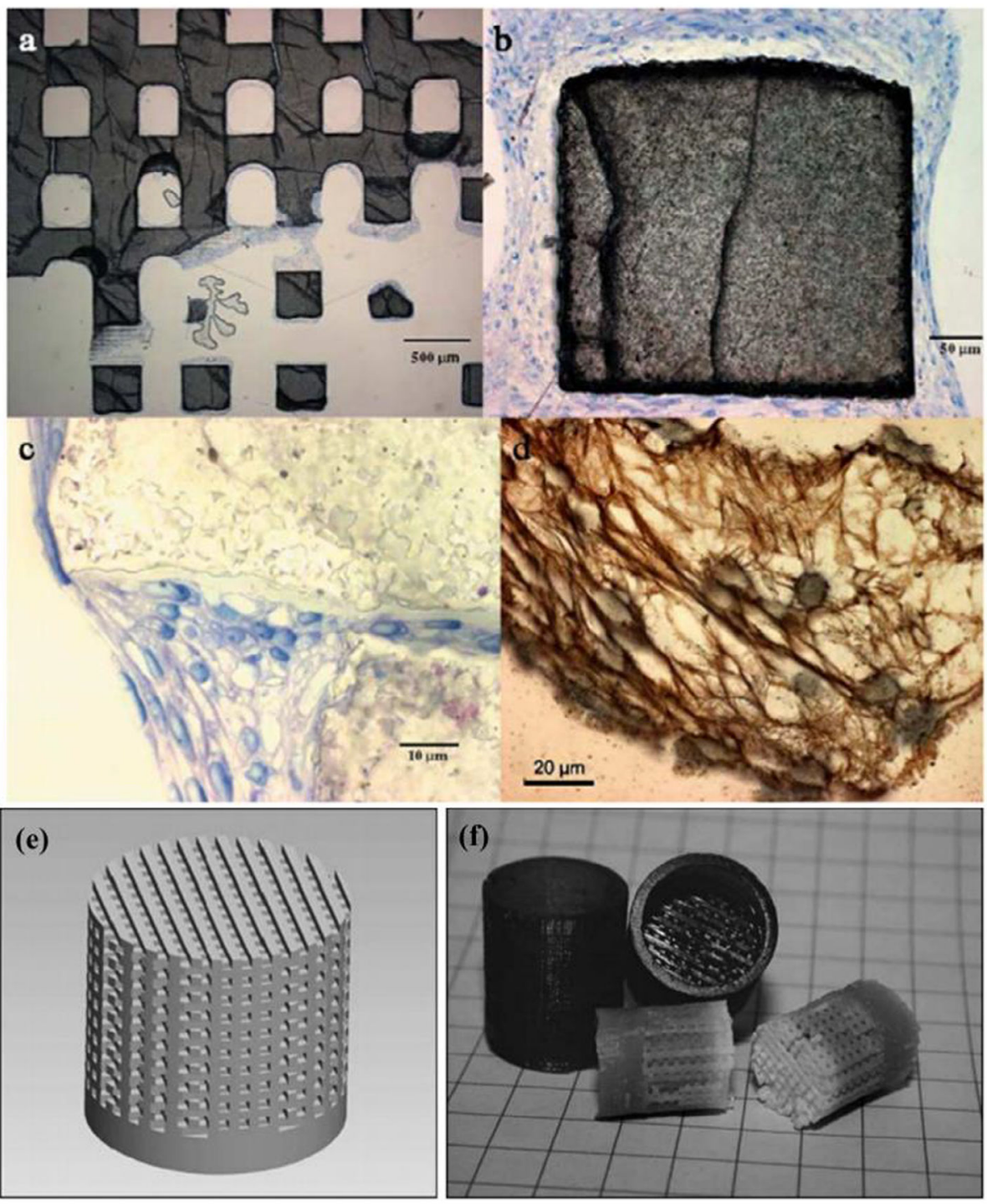

Fig. 14.

(a) Microstructure of MC3T3-E1 cells morphology on HA scaffold after 2 weeks (scaffolddark grey and cells-blue) (b) High magnification image showing the strut of HA scaffolds, which was totally attached with cells (blue/pink). (c) Microstructure of a crack between two struts, which was completely covered by MC3T3 cells and matrix generated by the cells (pink). (d) Images of collagen created by the cells (the microtome sectioning destructs the mineral scaffold). (e) The CAD design of the resin casting mold. (f) The produced casting mold by resin vat polymerization (black) and porous HA structure after the sintering (white) [100]. 

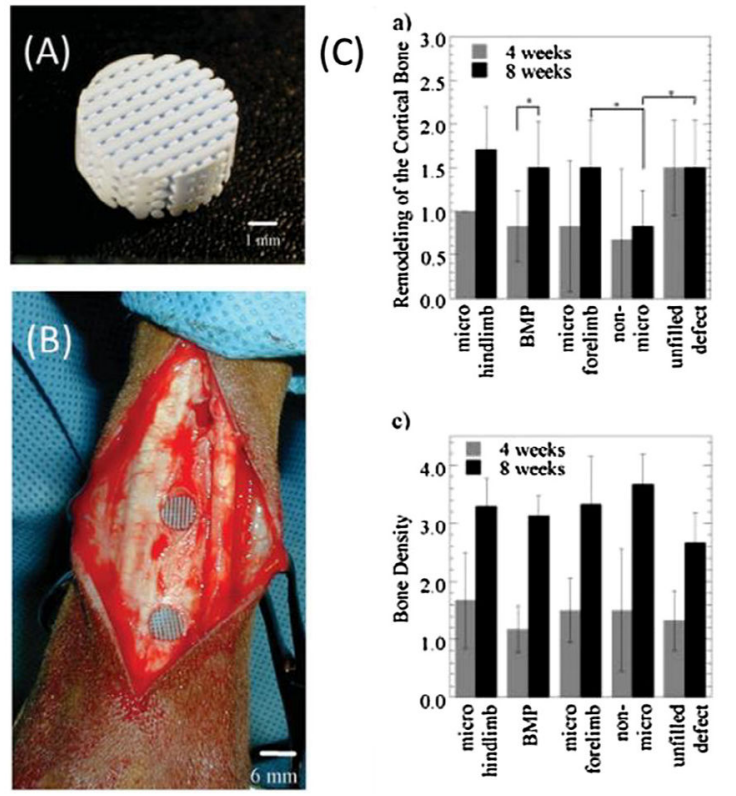
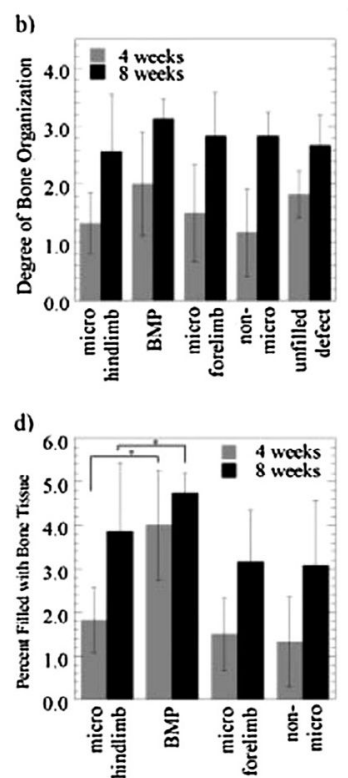

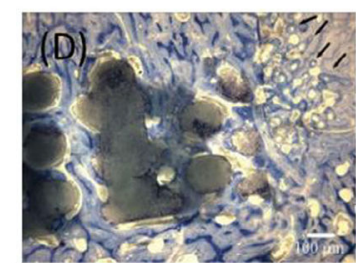

(E)
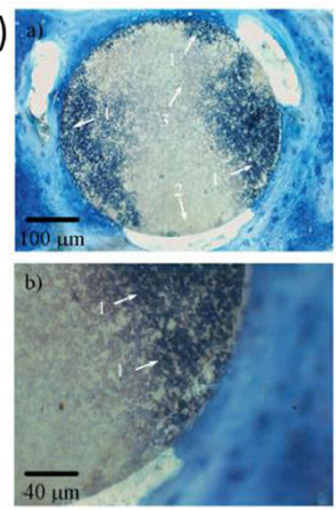

Fig. 15.

(A) The scaffold prepared by robocasting method. (B) The in vivo image of scaffold implantation in the goat metacarpal bone. (C) Effects of microporosity and BMP-2 on bone remodeling a), bone organization b), bone density c) and percent filled with bone tissue d). (D) and (E) are the BMP-2 added scaffold after 8 weeks and the microporous scaffold after 4 weeks with toluidine blue staining. Woven bone stains dark blue and lamellar bone stains light blue. Arrows indicate the bone remodeling in (D). (E-a) and (E-b) are in low and high magnifications. Arrows indicate (1) stained and (2) unstained regions and (3) regions where staining extends into the scaffold in (E) [105]. 

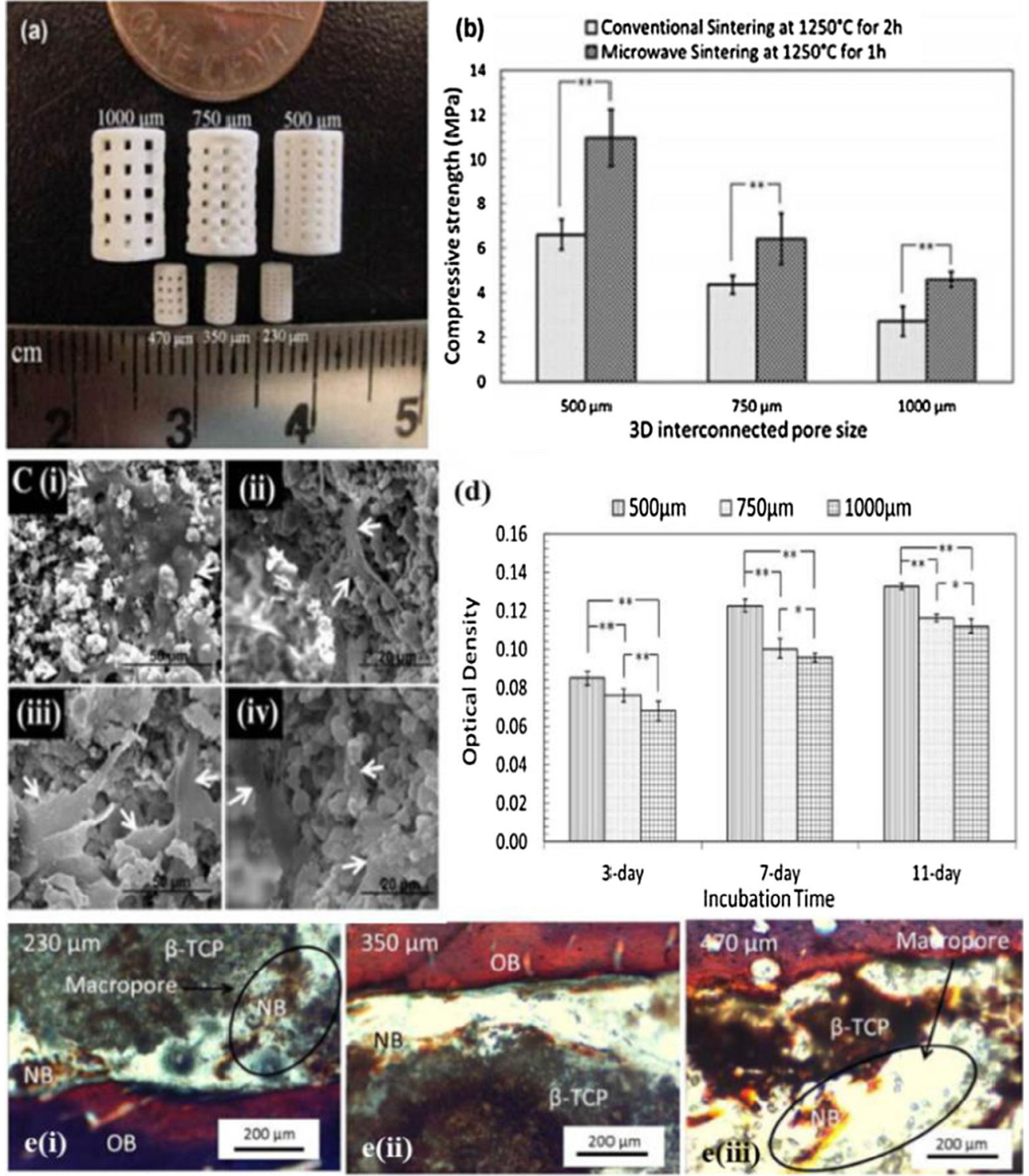

Fig. 16.

(a) The binder jetted scaffolds with different dimension and pore size. (b) Compressive strength of scaffolds with different designed pore size. (c) Cell morphology on scaffolds with $500 \mu \mathrm{m}$ pore size (i) \& (ii), and $750 \mu \mathrm{m}$ pore size (iii) \& (iv). (d) MTT assay of osteoblast cells on porous TCP scaffolds with different designed pore size $\left(^{* *} \mathrm{p}<0.05\right.$, ${ }^{*} \mathrm{p}>$ $0.05, \mathrm{n}=3$ ). (e) Histological images showing the new bone formation after two weeks (NB: new bone, OB: old bone) [47]. 

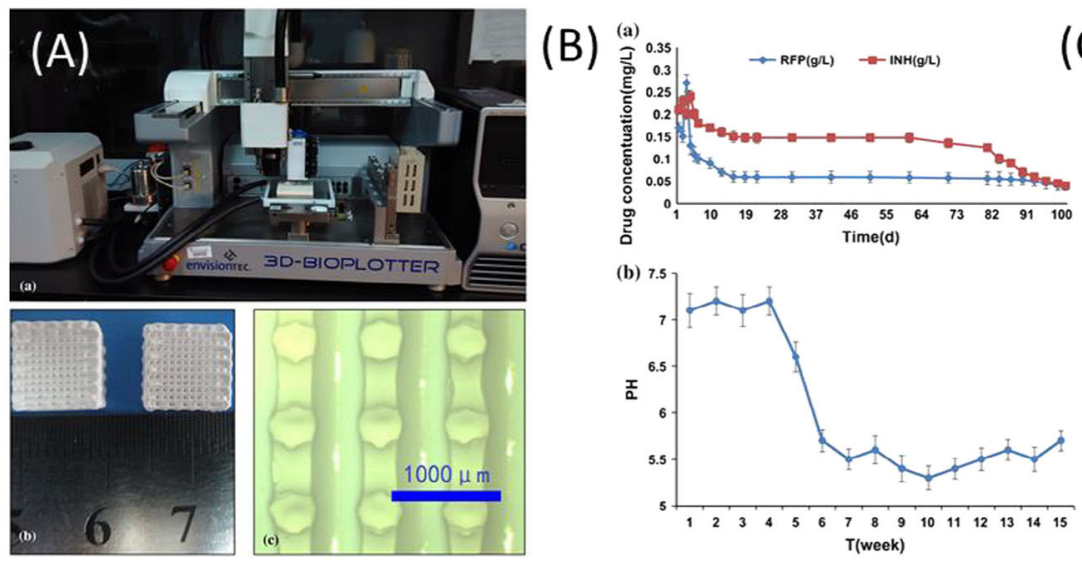

(C)
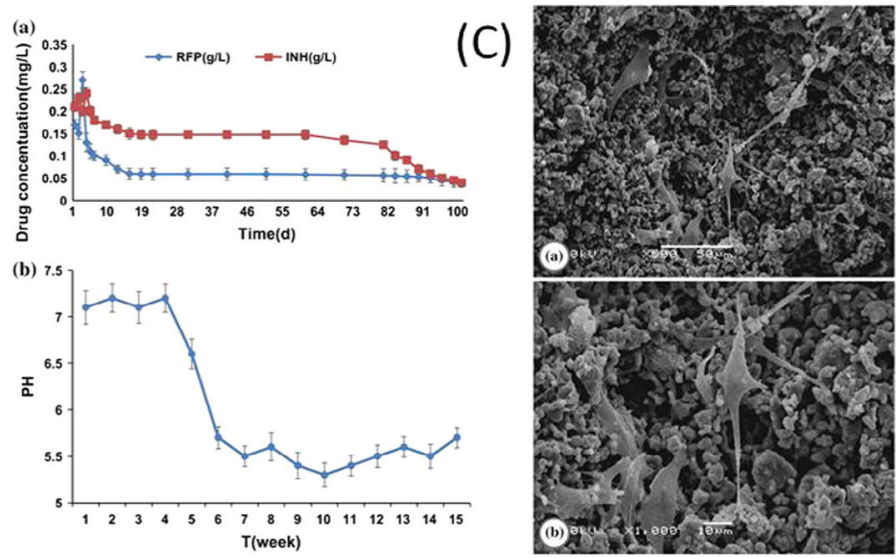

Fig. 17.

(A-a) The bioplotter, (B-a) scaffolds after the printing, and (C-c) the microstructure of scaffolds were shown in (A). (B-a) and (B-b) described the drug release till 100 days and the $\mathrm{PH}$ change during the release, respectively. (C) showed excellent biocompatibility of scaffolds using rBMSC cells [110]. 

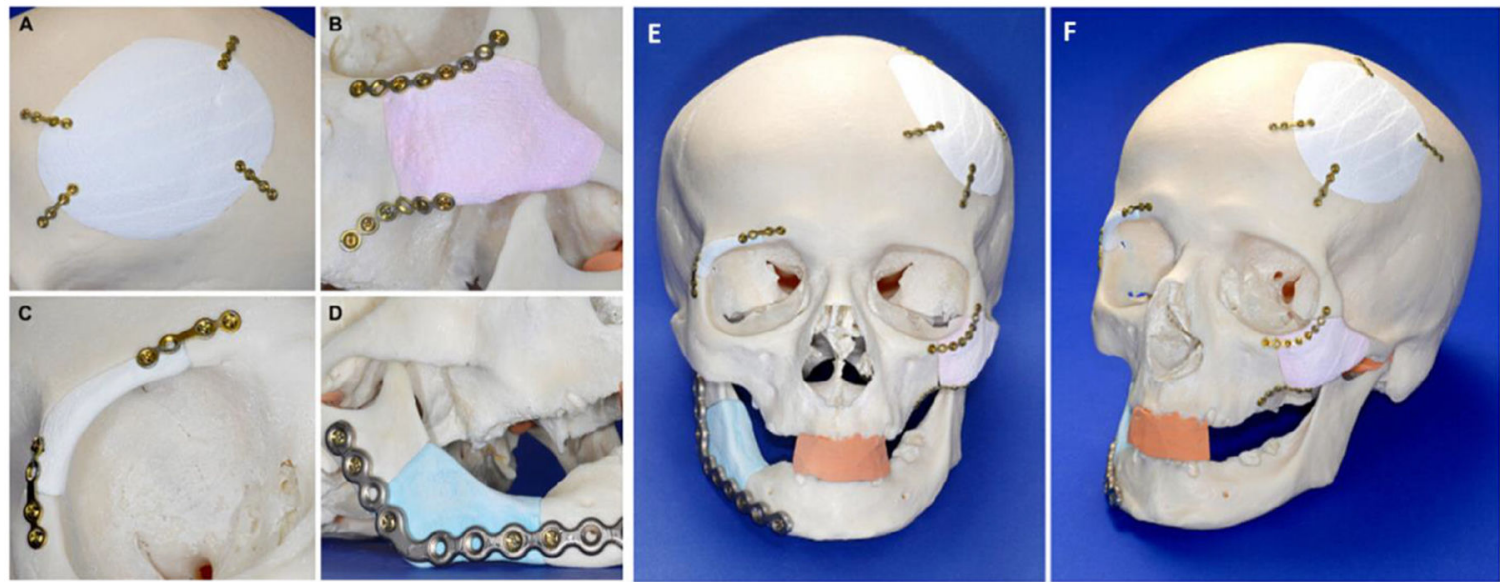

Fig. 18.

(A-D) The detail of the cranial and maxillofacial fixation area. (E and F) The general view of the whole cranial areas [114]. 

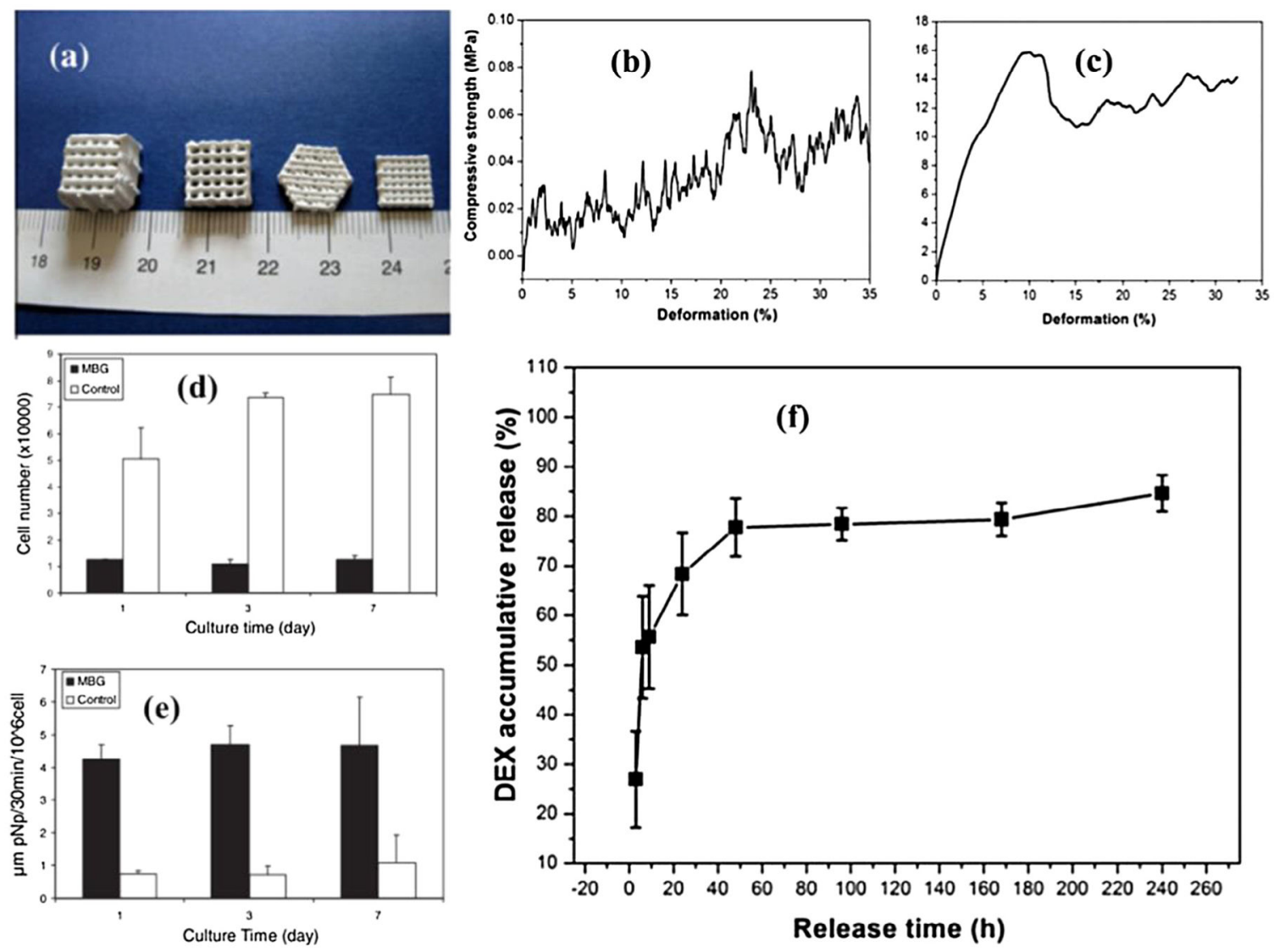

Fig. 19.

(a) Final scaffolds with different pore designs. Comparison of compressive strength prepared by polyurethane template (b) and binder jetting (c). The proliferation (d) and ALP assay (e) using human bone marrow stromal cells (hBMSCs) on scaffolds. The accumulative release of dexamethasone (DEX) on the scaffold (f) [125]. 

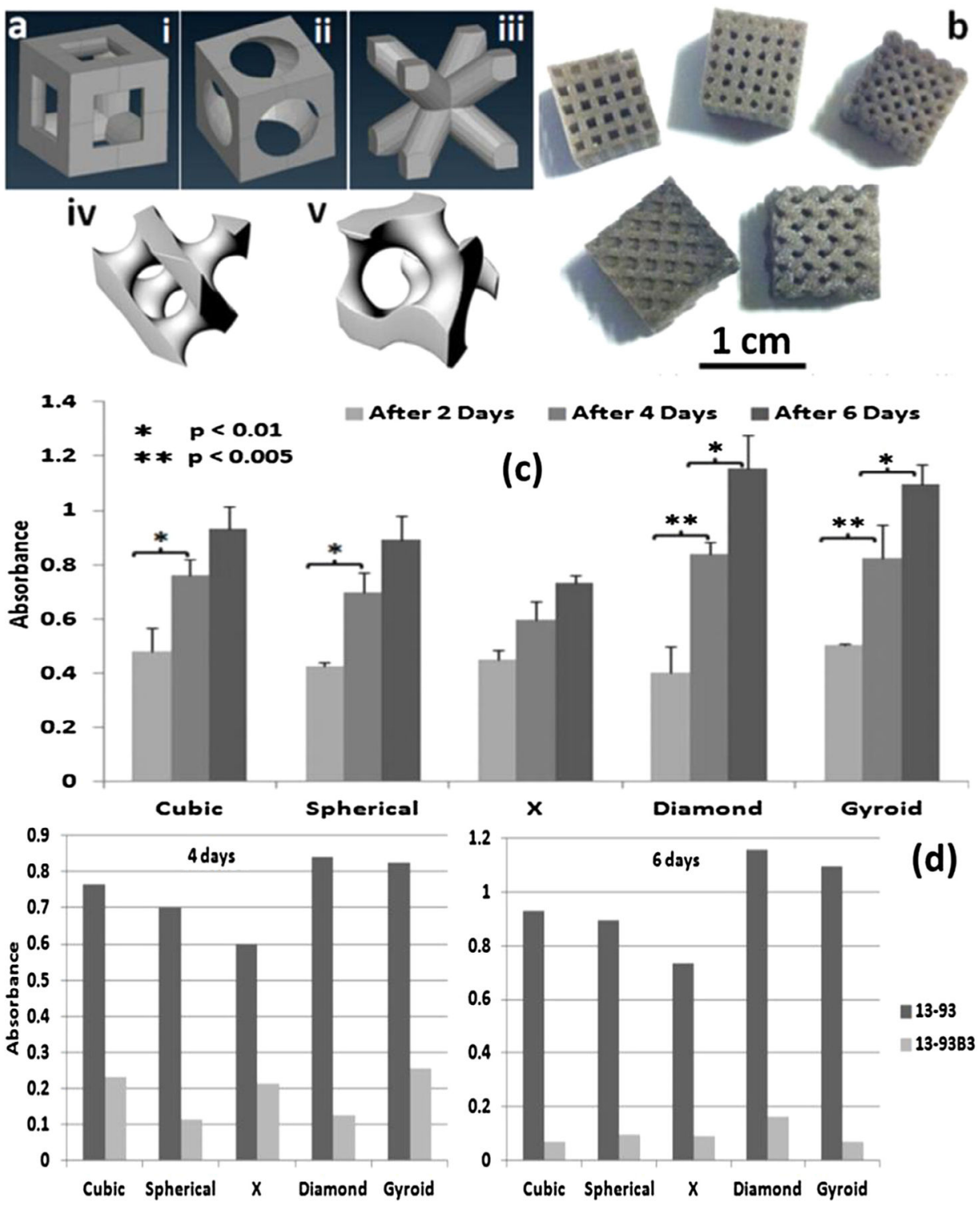

Fig. 20.

(a-i) cubic, (a-ii) spherical, (a-iii) X, (a-iv) diamond and (a-v) gyroid were different designs for porous bioglass scaffolds and their final parts after laser sintering were showed as (b). The MTT assay (c) showed diamond and gyroid designs had improved cell growth and (d) indicated that 13-93B3 had a lower bioactivity than 13-93 [130]. 

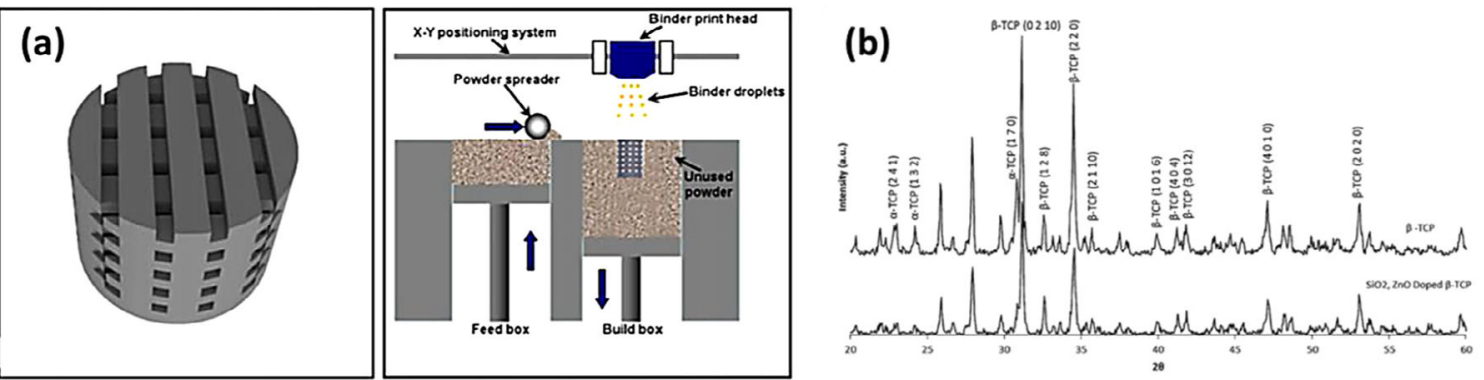

\begin{tabular}{|c|c|c|c|c|}
\hline $\begin{array}{l}\text { Interconnected } \\
\text { channel size }\end{array}$ & $\begin{array}{l}\text { Scaffold } \\
\text { type }\end{array}$ & $\begin{array}{l}\text { Apparent density } \\
\text { of struts }\end{array}$ & $\begin{array}{l}\text { Total open } \\
\text { pore volume }\end{array}$ & $\begin{array}{l}\text { Designed pore } \\
\text { volume }\end{array}$ \\
\hline $750 \mu \mathrm{m}$ & $\begin{array}{l}\text { Pure } \\
\text { Doped }\end{array}$ & $\begin{array}{l}91.2(1.7) \\
92.2(4.1)\end{array}$ & $\begin{array}{l}44.31 \% \\
43.31 \%\end{array}$ & $35.51 \%$ \\
\hline
\end{tabular}
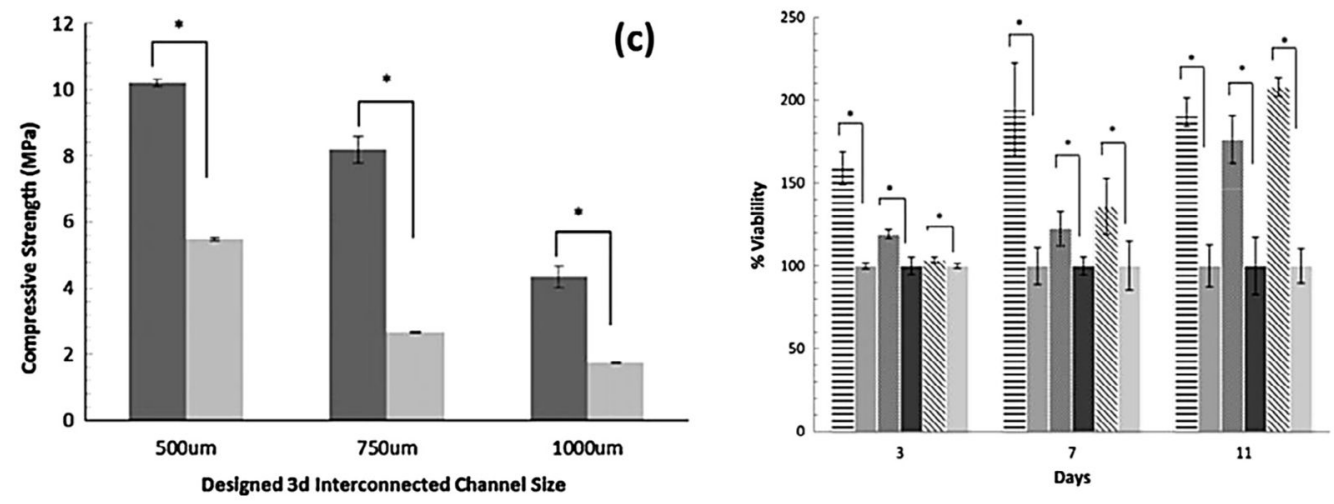

(d)

Fig. 21.

(a) The CAD design of the scaffold and the schematic of the 3D print machine for making interconnected porous scaffold. (b) The XRD characterization of pure and doped TCP scaffolds after sintering at $1250^{\circ} \mathrm{C}$. Table was the porosity information for different pore size design after sintering(c) The compressive strength of sintered scaffolds (dark: doped scaffolds, grey: pure TCP). (d) The MTT assay after 3, 7 and 11 days of cell culture using osteoblast cells [42]. 

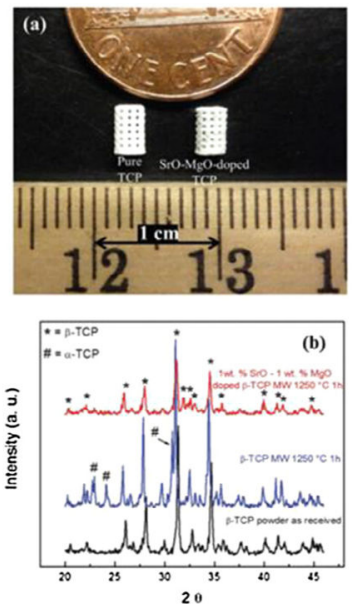

(c)
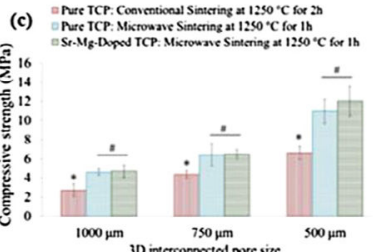

Fig. 22. implantation $[44,138]$.

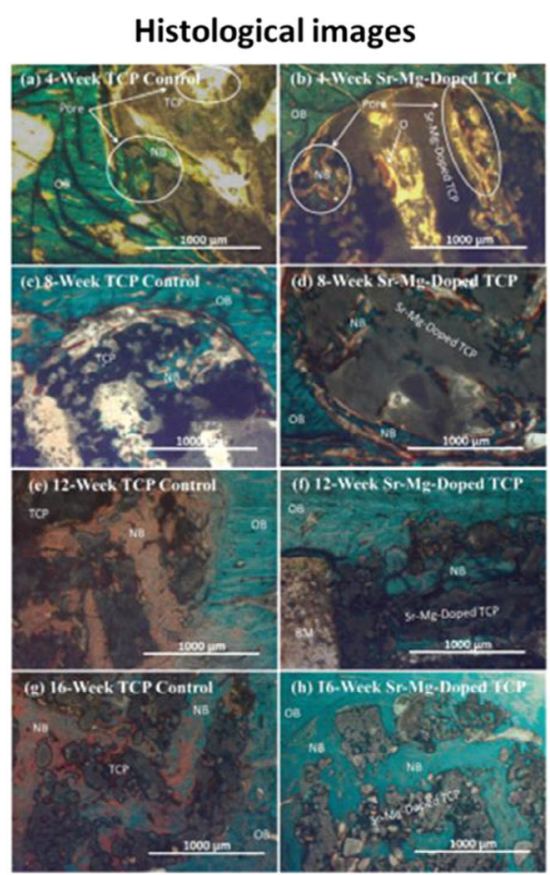

(d) $=$ Pure TCP $\quad$ Sr-Mg Doped TCP

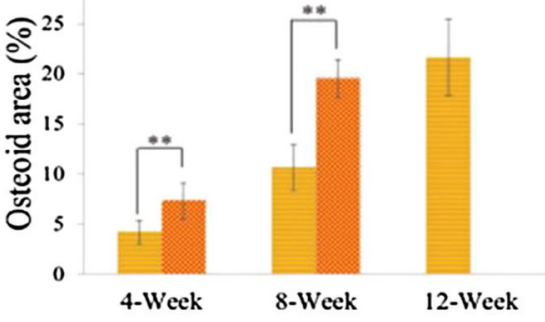

(e) $\quad$ Pure TCP $=\mathrm{Sr}-\mathrm{Mg}$ Doped TCP

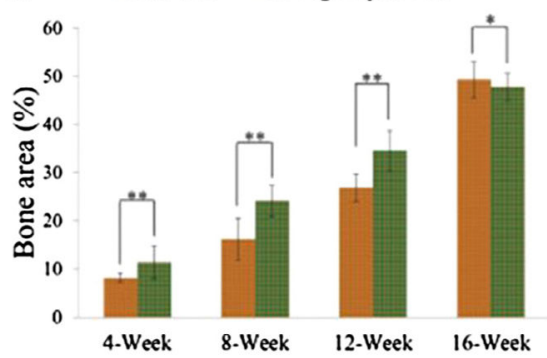

(a) The image showing sintered scaffolds by binder jetting method. (b) The XRD peaks after different sintering conditions for pure $\beta$-TCP and SrO/MgO doped TCP. (c) The compressive strength of pure and doped scaffolds by different pore size designs and sintering conditions. Histological images showed scaffold (dark grey/black), osteoid formation (orange/red) and mineralized bone (green/bluish) after 4, 8, 12 and 16 weeks of implantation. (d) and (e) showed the osteoid and new bone formation after 4 to 16 weeks of 

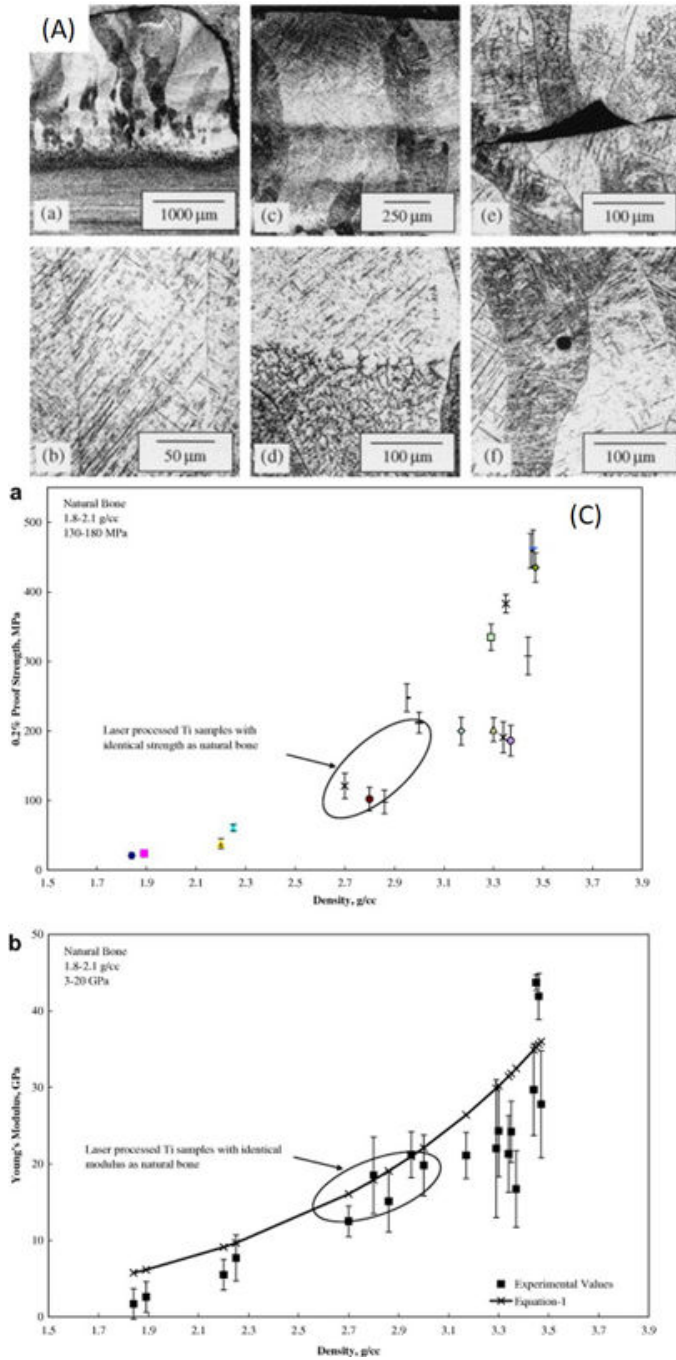

(B)

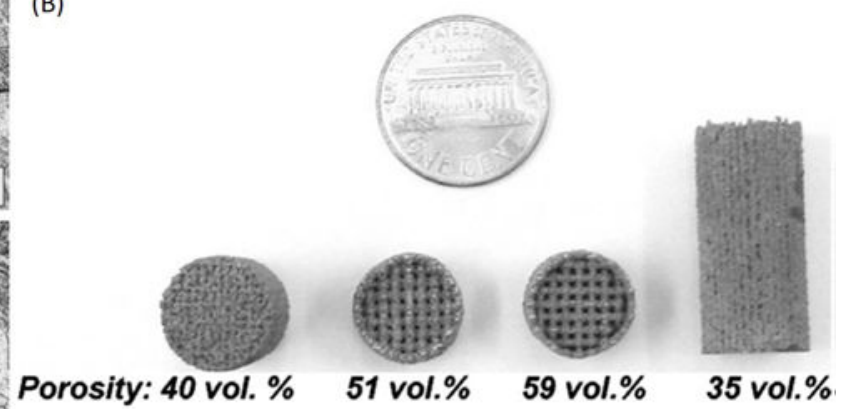

(D)
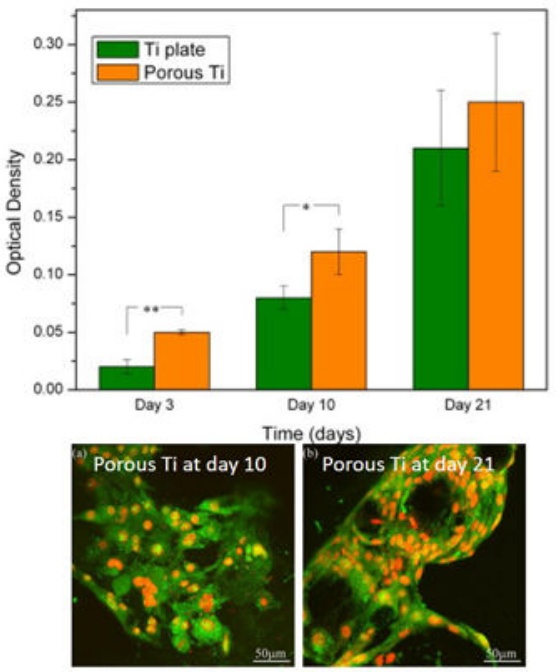

(days)

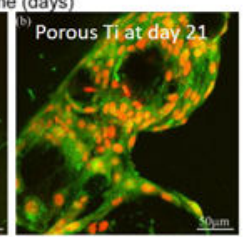

Ti plate at day 10

Tiplate at day 21

Fig. 23.

(A) General microstructure of laser additively manufactured Ti-6Al-4V alloy [56]. (B) LENS $^{\mathrm{TM}}$ processed porous Ti structures, $(\mathrm{C}-\mathrm{a})$ the effects of density on $0.2 \%$ proof strength, and (C-b) comparison of theoretically calculated and experimental effective moduli of porous Ti samples [61]. (D) MTT assay of Ti plate and porous Ti at day 3, day 10, and day 21 as well as confocal microscopy images of Ti plate and porous Ti at day 10 and day 21 [141].

Prog Mater Sci. Author manuscript; available in PMC 2019 August 12. 

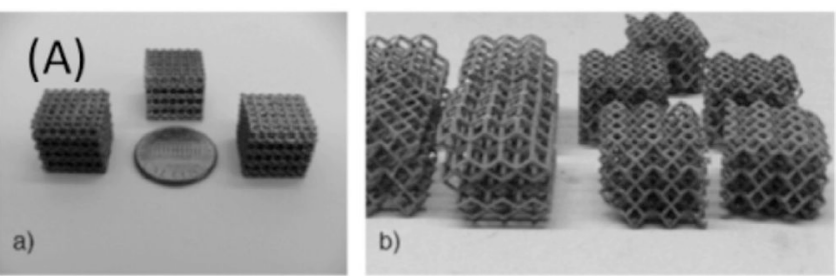

b)
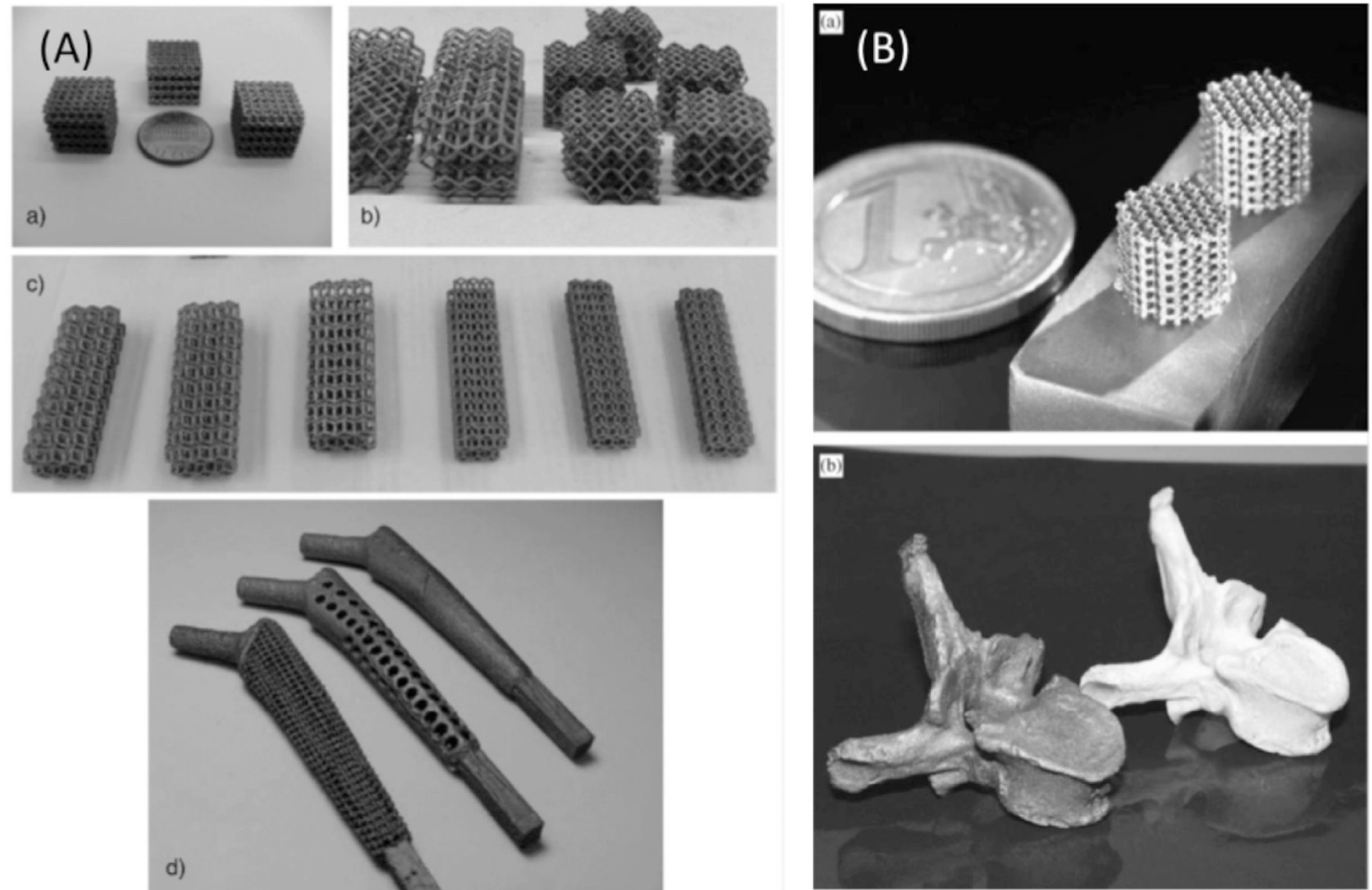

d)

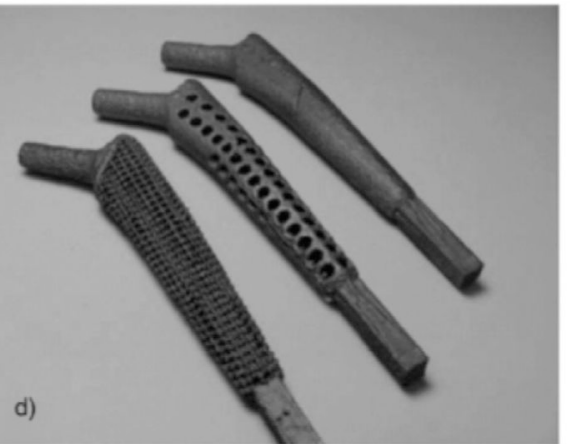

Fig. 24.

(A) Different Ti-6Al-4V structures manufactured by E-beam melting [143] and (B) Mesh Ti64 alloy structures and a replica of human vertebrae fabricated by SLS/DLF technique [149]. 

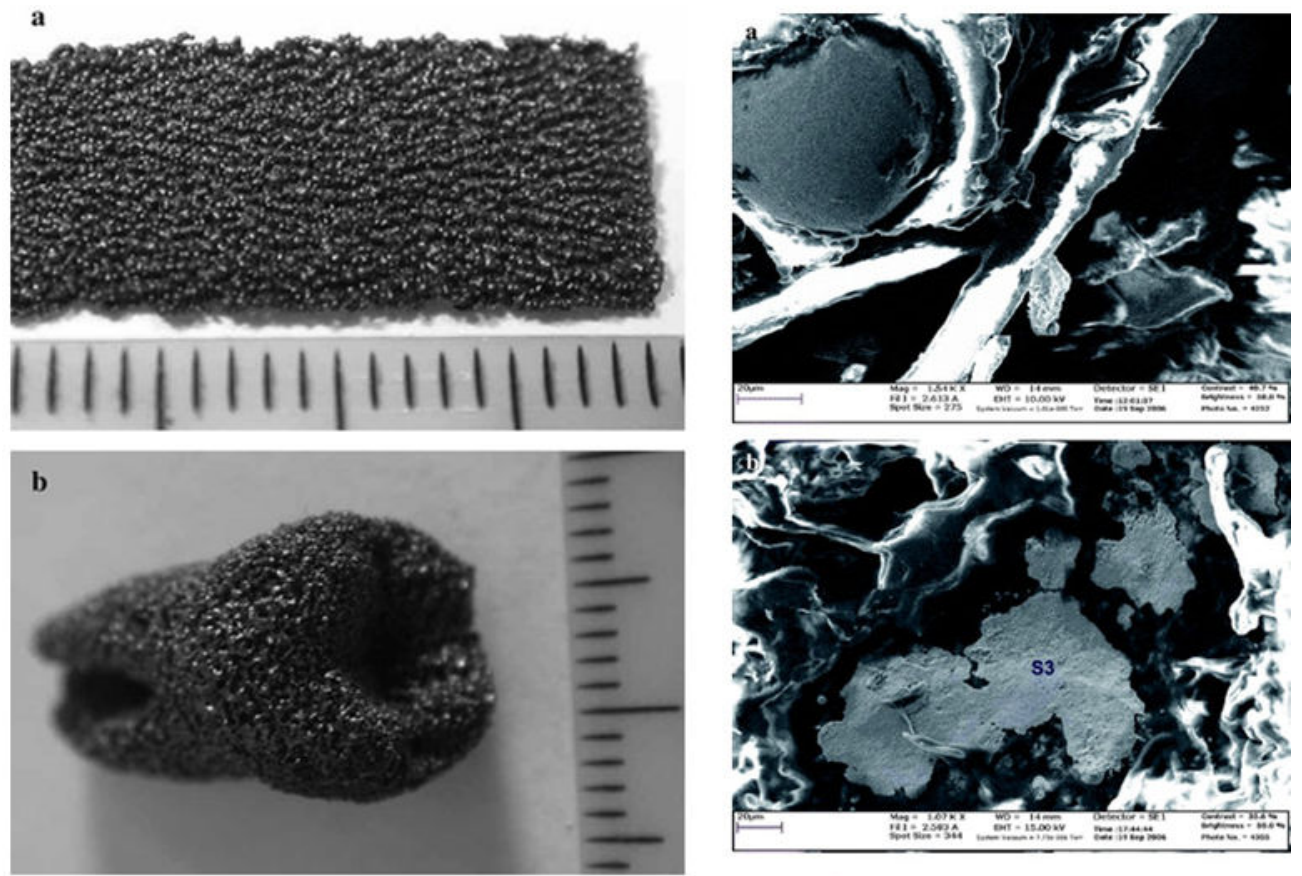

(A)
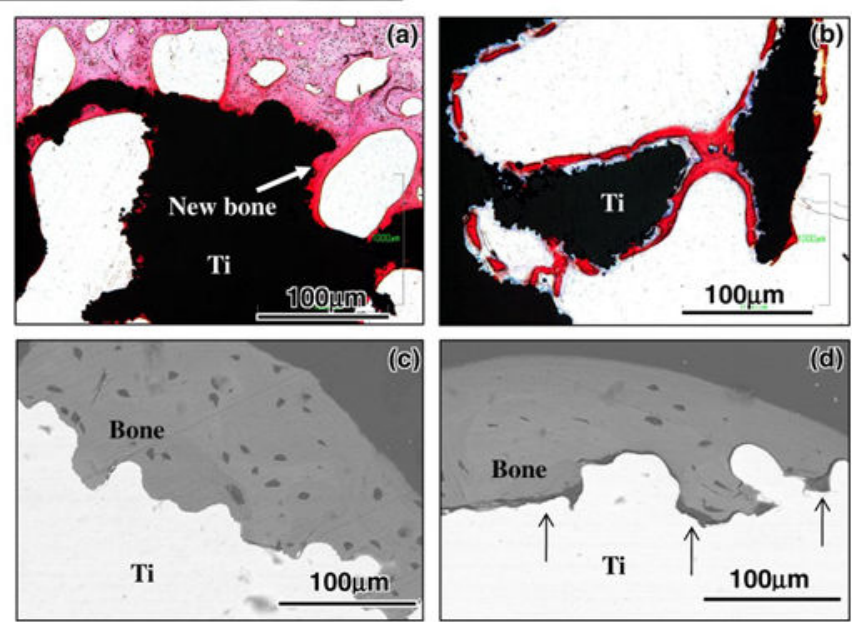

(B)

Fig. 25.

(A) NiTi porous implant fabricated by SLS/SLM (left) and the same implant showing bone tissue integration after 3 months in a rabbit model [151] and (B) new bone formation in a porous Ti alloy implant after 12 weeks [153]. 


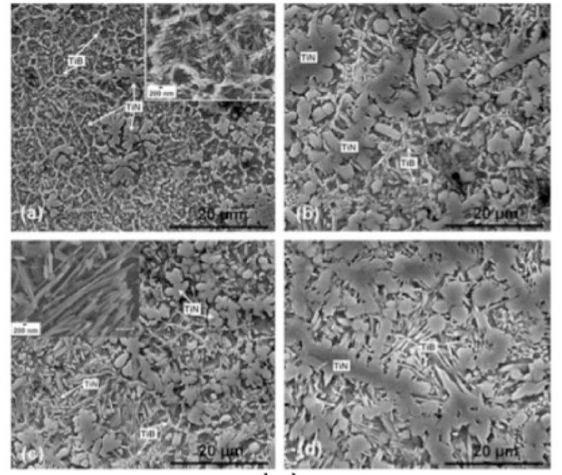

(a)
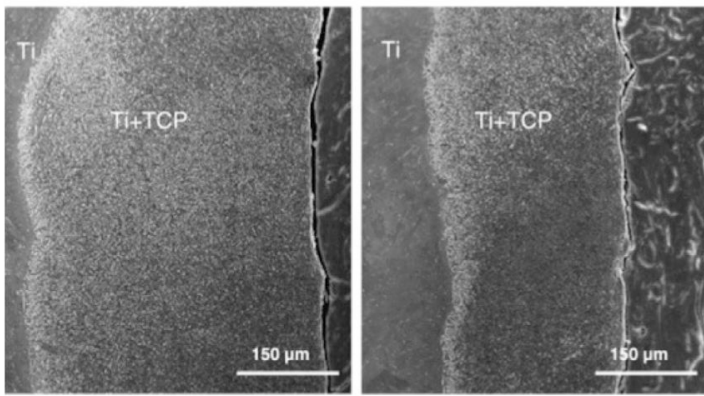

(b)
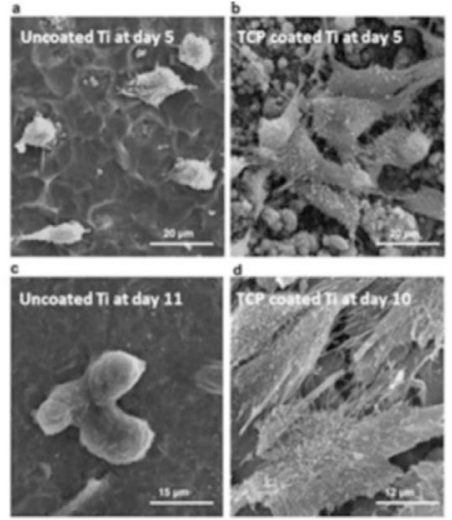

(c)

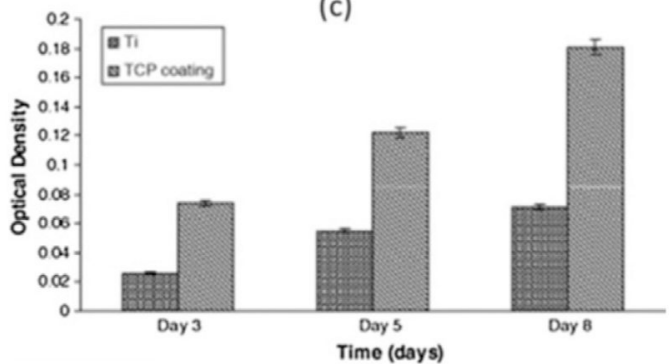

(d)

Fig. 26.

(a) Ti64 alloy reinforced with BN using LENS ${ }^{\mathrm{TM}}$ [160]. (b) LENS ${ }^{\mathrm{TM}}$ processed tricalcium phosphate (TCP) coating on Ti, (c) osteoblast morphology on uncoated Ti and TCP coated Ti after 5 and 10 days of cell culture, and (d) MTT assay of uncoated Ti and TCP coated Ti after 3, 5, and 8 days of cell culture [169]. 

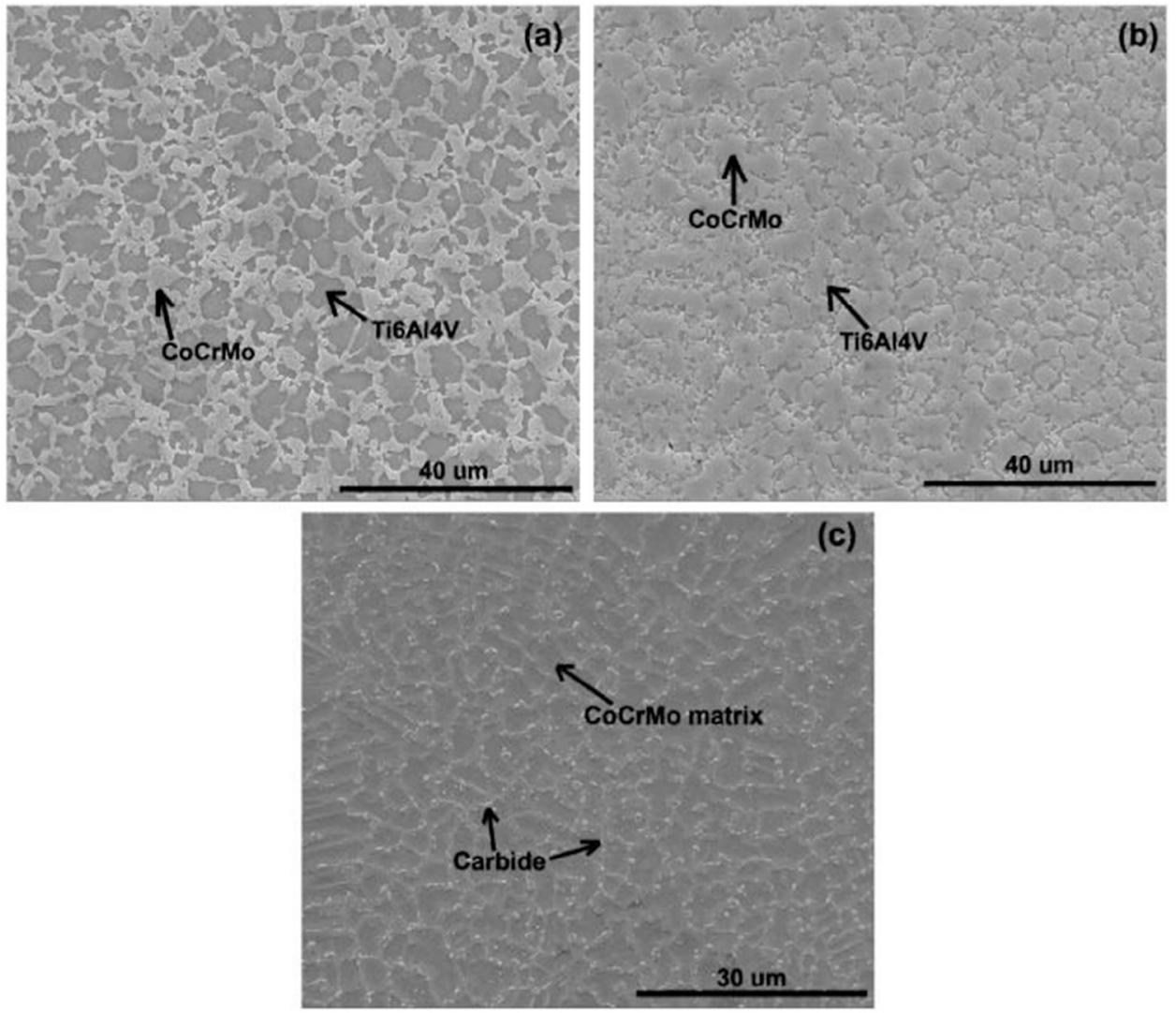

Fig. 27.

CoCrMo-Ti6A14V Structures fabricated using LENS ${ }^{\mathrm{TM}}$ (a) with 50\% CoCrMo alloy in Ti64 (b) with 86\% CoCrMo alloy in Ti64 and (c) CoCrMo alloy [64]. 

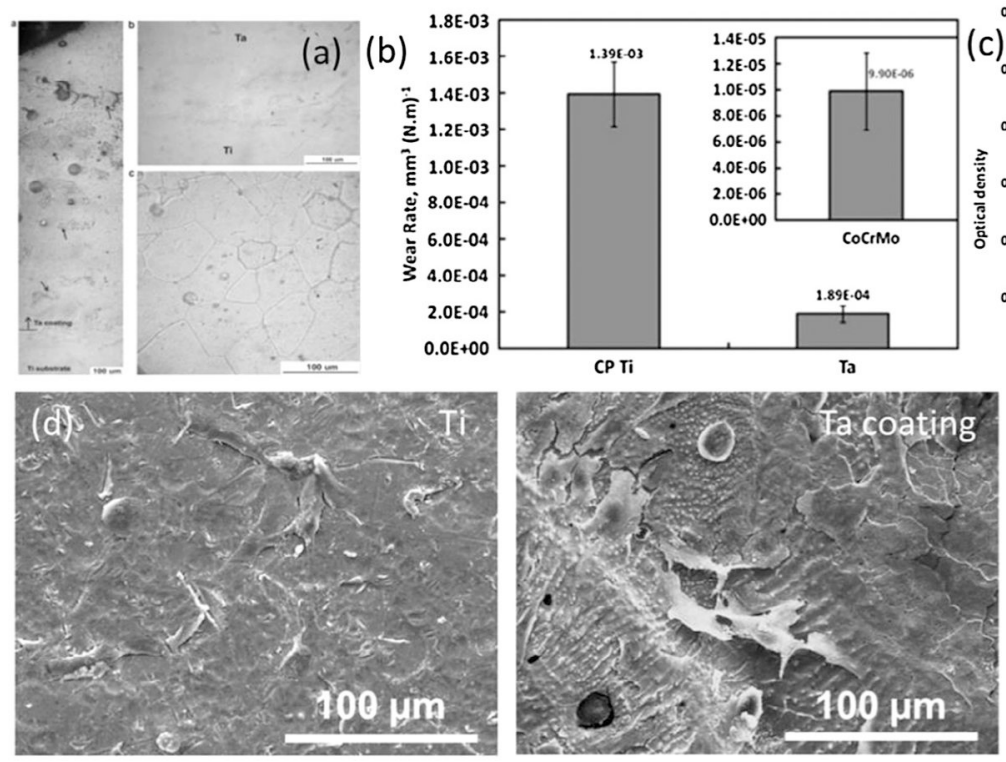

Fig. 28.

(a) Microstructure of LENS ${ }^{\mathrm{TM}}$ processed Tantalum on Ti [173]. (b) Wear performance of LENS $^{\text {TM }}$ processed Ta coatings on $\mathrm{Ti}$ [174]. (c) MTT assay of $\mathrm{Ti}$, Ta coating, and $\mathrm{Ta}+\mathrm{MgO}$ coating after 3, 7, 11 days of osteoblast cell culture as well as (d) osteoblast morphology of $\mathrm{Ti}$, Ta coating, and $\mathrm{Ta}+\mathrm{MgO}$ coating after 7 days of osteoblast cell culture [175]. 

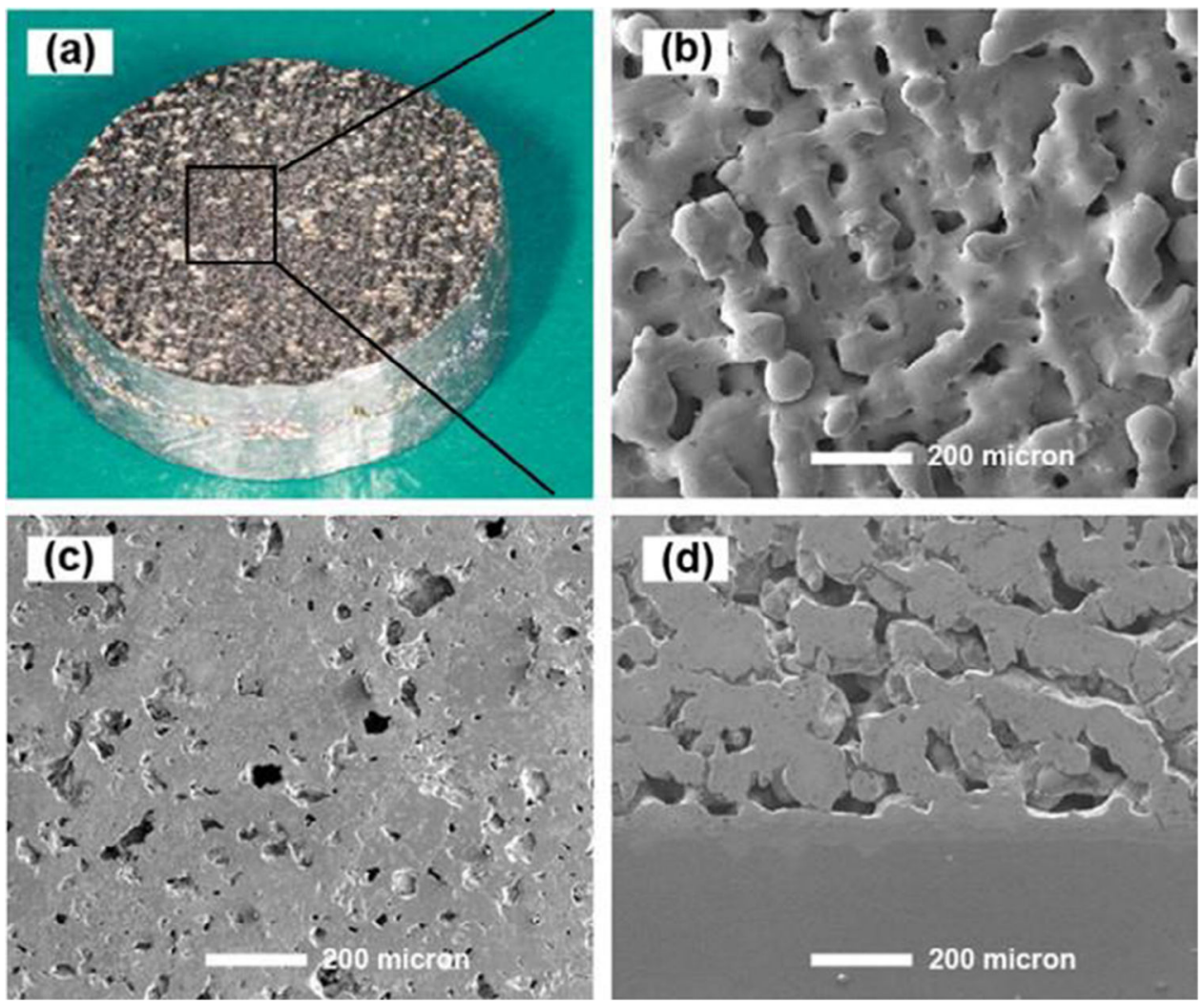

Fig. 29.

SLM processed porous Niobium coating on Ti [177]. 


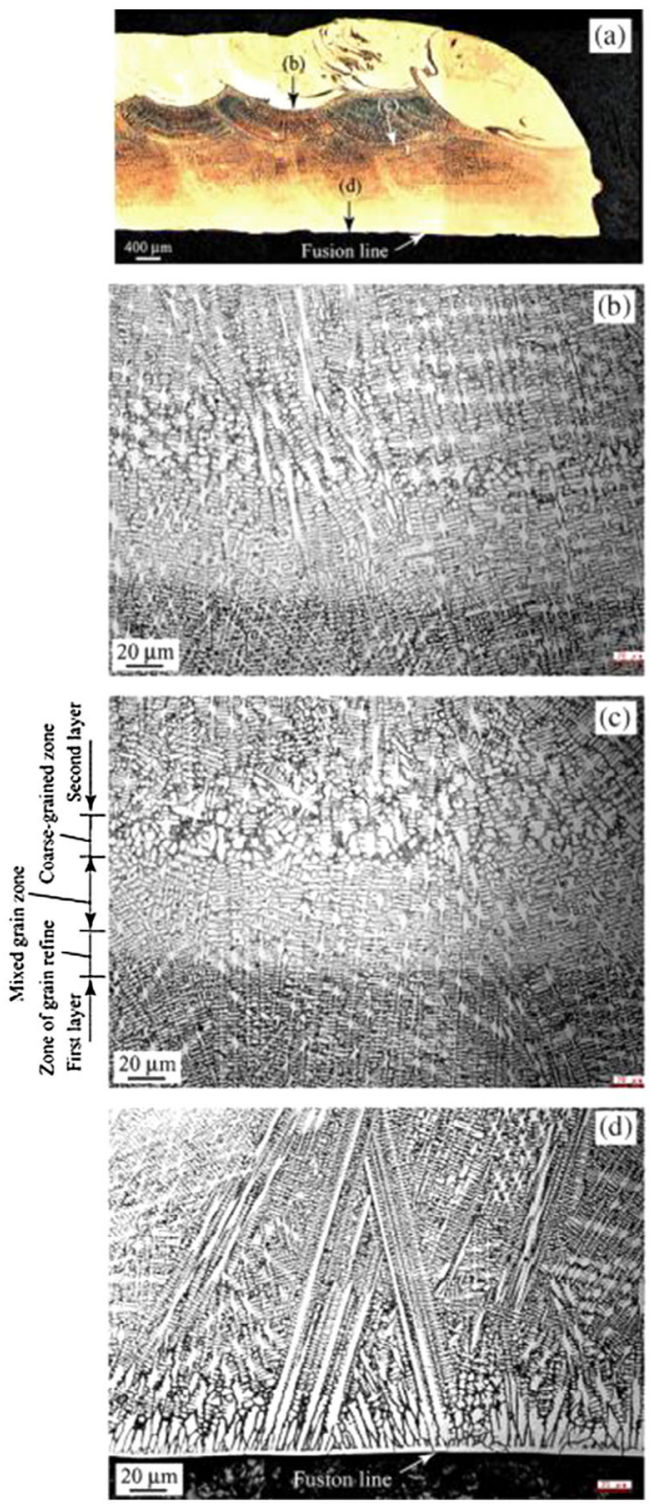

Fig. 30.

Functionally graded WC-Co composites fabricated by laser cladding [179]. 
(A)

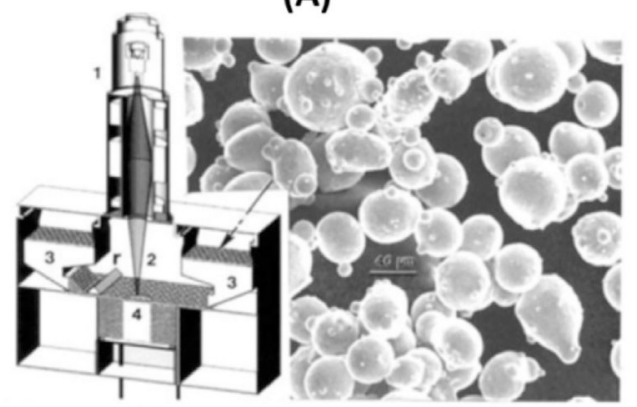

(a)

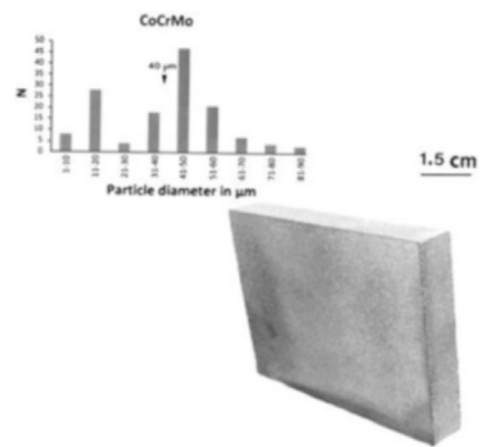

(c) (b)

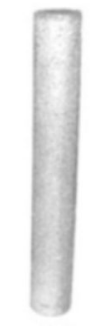

(d)
(B)

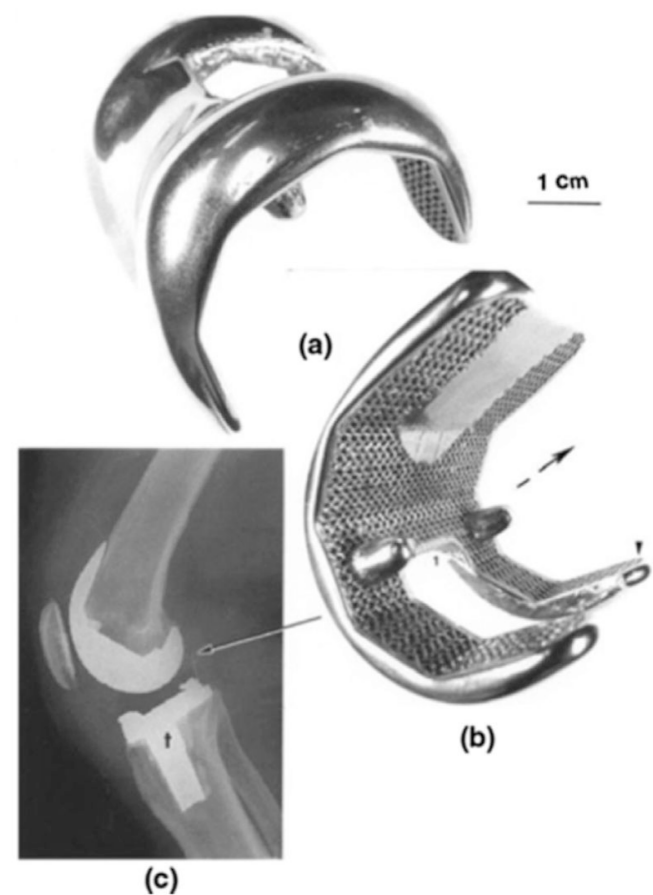

Fig. 31.

E-beam process for CoCrMo alloy: Set (A) E-beam process key feature and powder distribution and dense E-beam processed samples and set (B) Porous femoral knee E-beam processed components [181]. 

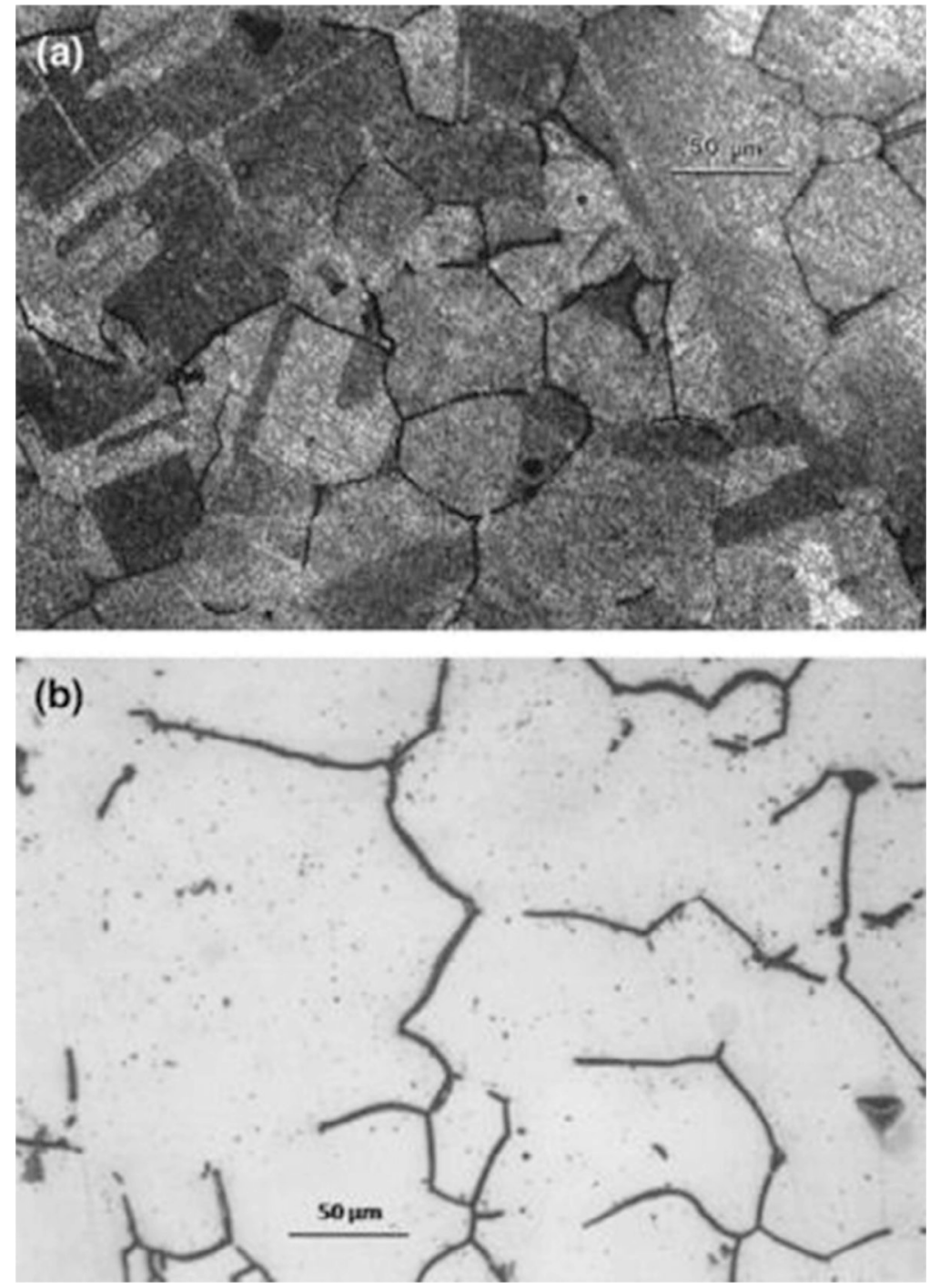

Fig. 32.

Microstructure showing annealing twins in CoCrMo after E-beam processing and annealing [181]. 
(1)

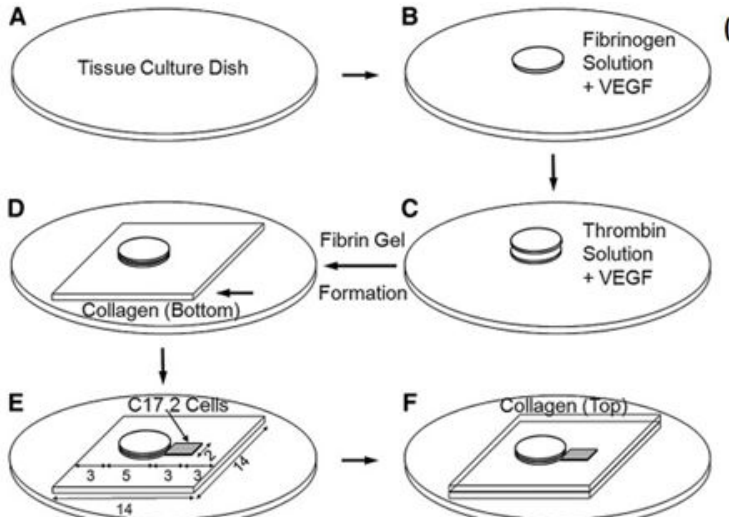

(3)

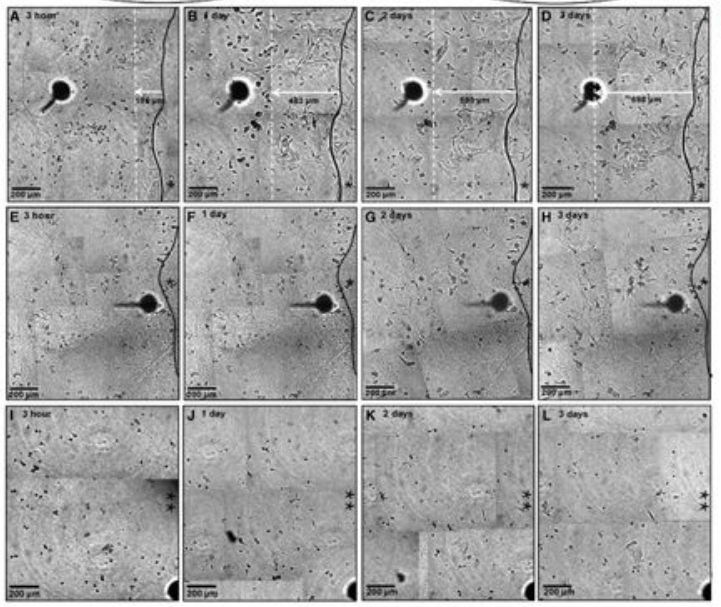

(2)

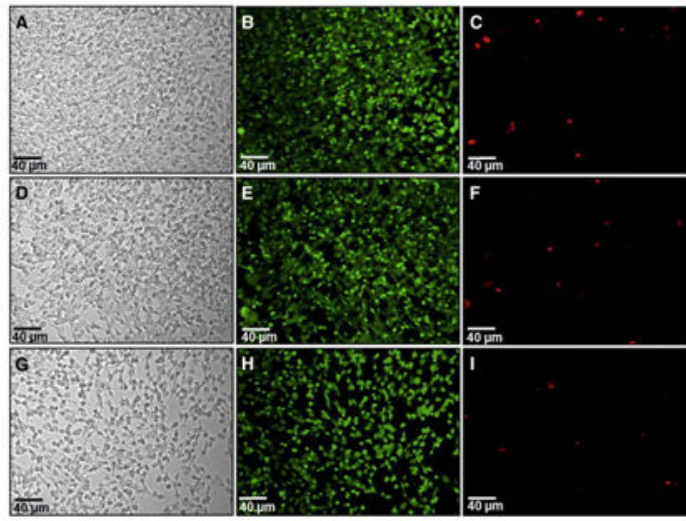

(4)

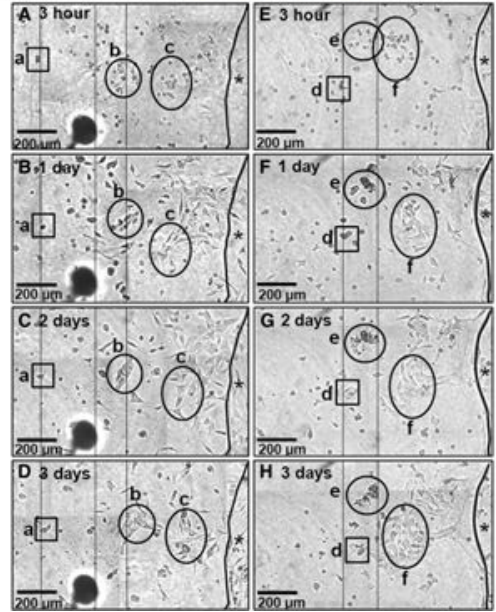

Fig. 33.

(1) The bioprinting of VEGF released fibrin gel, collagen and C17.2 cells. (2) C17.2 cell proliferation on three concentrations of collagen scaffolds after 3 days of culture $(\mathrm{A}, \mathrm{B}, \mathrm{C}$ 1.74 mg/mL; D, E, F - 1.16 mg/mL; G, H, I - 0.87 mg/Ml; A, D, G - Bright-field cell images; B, E, H - Calcein AM live fluorescence images and C, F, I - ethidium-homodimer (EthD-1) dead fluorescence). (3) Time lapse photos showing cell migration and proliferation of fibrin gel containing VEGF loaded collagen scaffolds (A-D), fibrin gel containing VEGF absent collagen scaffolds (E-H) and fibrin gel matrix absent VEGF loaded collagen scaffolds (I-L) at $3 \mathrm{~h}$, day 1 , day 2 and day 3 . The range of the affected cells displaying these morphological changes (a white vertical dot line) was broadened with time lapse.

Fibrin gel containing VEGF loaded collagen scaffolds showed cluster cells formation and its area increased as the time went by (4) Time lapse photos showing effects of VEGFcontaining fibrin gel (left column-fibrin gel contained and right column-fibrin gel absent) on cells at 3 h, day 1, day 2 and day 3. C17.2 cells (black square, a and d) moved toward the border (black thick line) of the fibrin gel (*) over the observation time (3 days). Cells in black circles (b, c, e, and f) continued proliferation and differentiation with formation of cell clusters during migration [189]. 

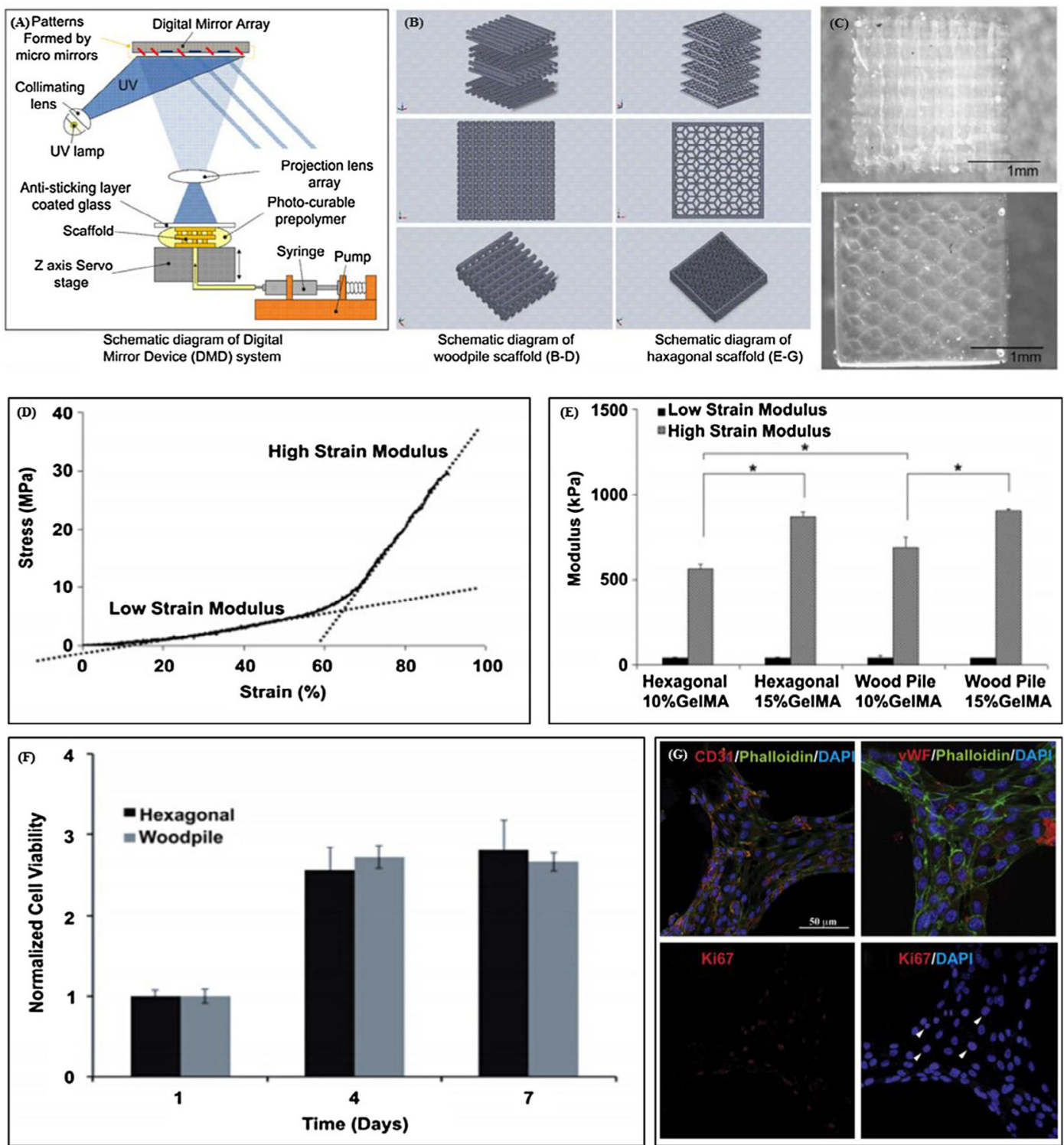

Fig. 34.

(A) Schematic of projection stereolithography. (B) CAD designs of woodpile hexagonal scaffolds. (C) Final woodpile and hexagonal scaffolds by stereolithography (D) and (E) showed the low strain and high strain modulusof different scaffolds. (F) HUVECs viability after 1, 4 and 7 days of culturing on woodpile and hexagonal scaffolds. (G) Immunofluorescence micrographs showing biological functionality of cells using different markers [196]. 
(A)

(B)

(D)

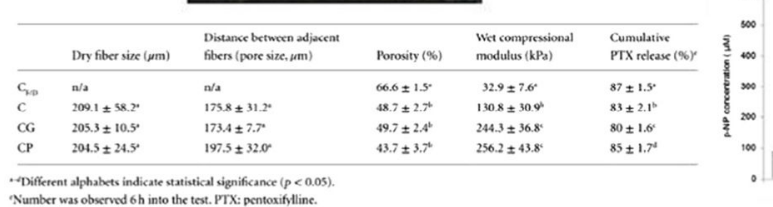

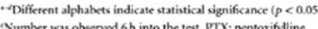

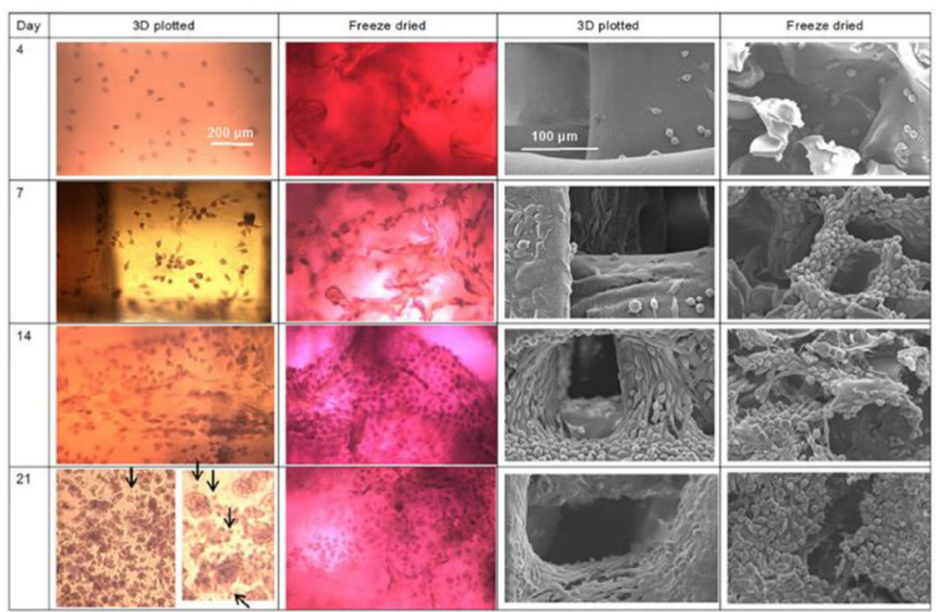

(C)
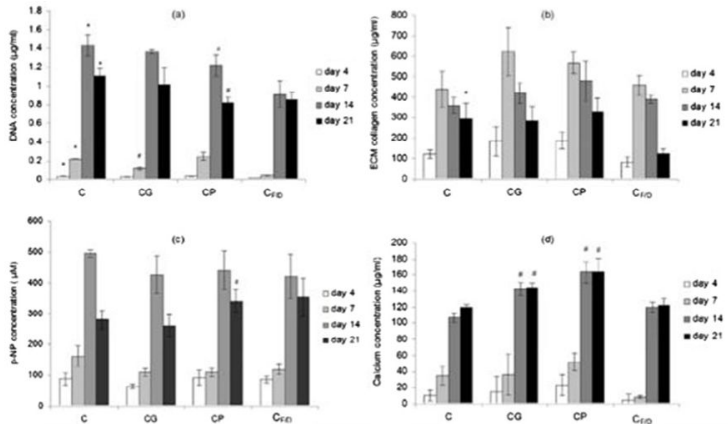

(E)

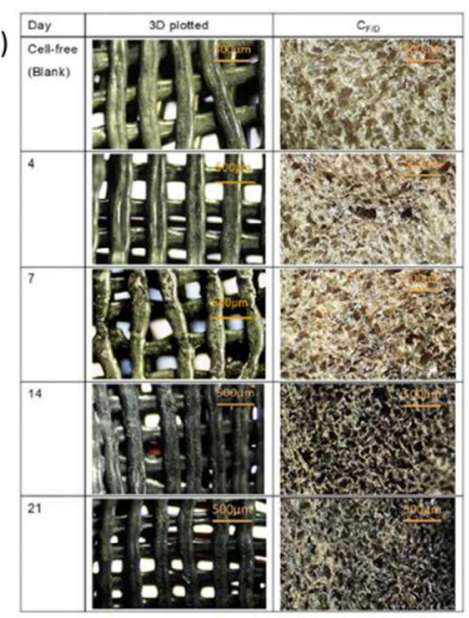

Fig. 35.

(A) Porous chitosan scaffolds after the printing. (B) Physical properties of scaffolds prepared by different chitosan based materials (C) DNA concentration (C-a), ECM collagen concentration (C-b), ALP concentration (C-c) and calcium concentration (C-d) for in vitro characterization using osteoblast cells till day 21. (D) H\&E and SEM images showing the cell morphology. (E) Von Kossa staining images showing the mineralization by black [200]. 
(1)

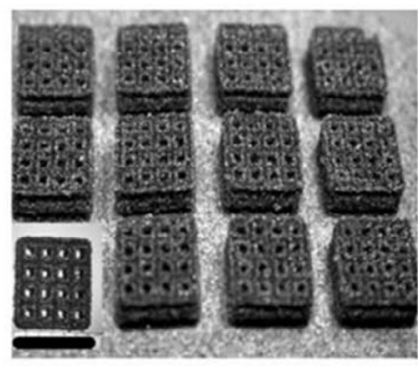

(3)
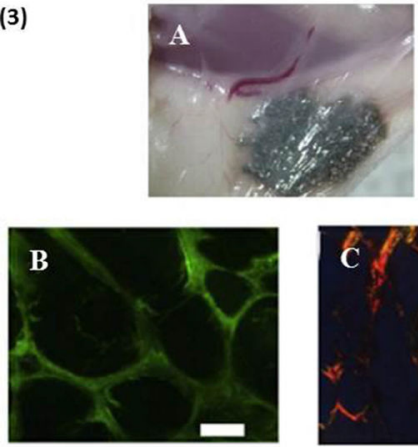

(2)

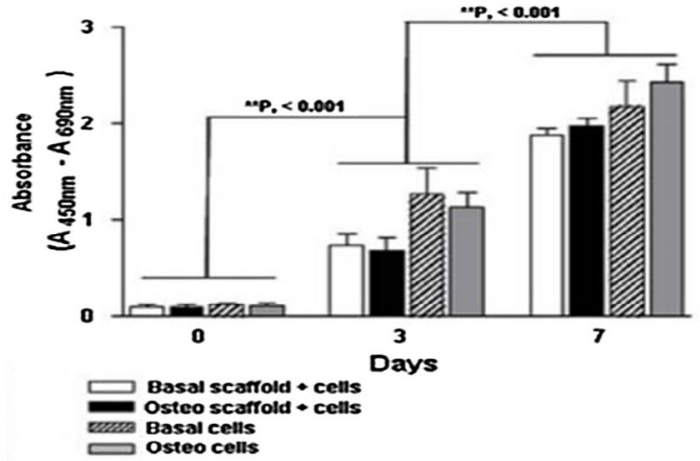

(4)
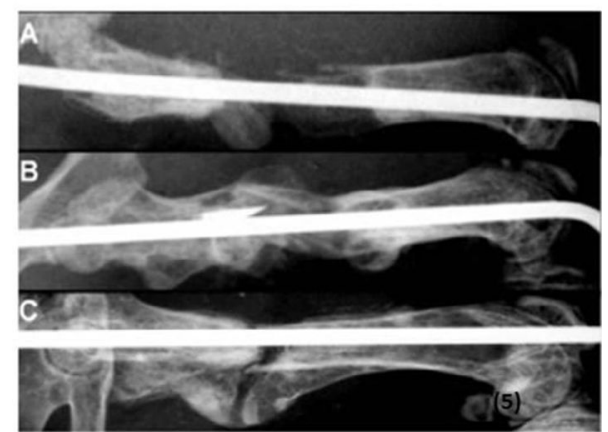

Fig. 36.

(1) The SLS manufactured porous PLA scaffolds (2) Femur-derived cells proliferation in basal medium ( $a-$ MEM/10\% FCS), osteogenic culture medium (a -MEM/10\% FCS with $100 \mathrm{lM}$ ascorbate and $10 \mathrm{nM}$ dexamethasone), basal medium on PLA scaffolds and osteogenic culture medium on PLA scaffolds after 0, 3 and 7 days. (3-A) Femur-derived cells loaded PLA scaffold in vivo. (3-B) and (3-C) were fluorescence image showing viable fetal femur-derived cells via Vybrant CFDA SE Cell Tracer Kit and polarized light micrograph showing newly formed collagen, respectively. X-ray images of defect site without the PLA scaffold (4-A) and with the PLA scaffold (4-B) and with the femur-derived cells loaded PLA scaffold (4-C) after 28 days of implantation [211]. 


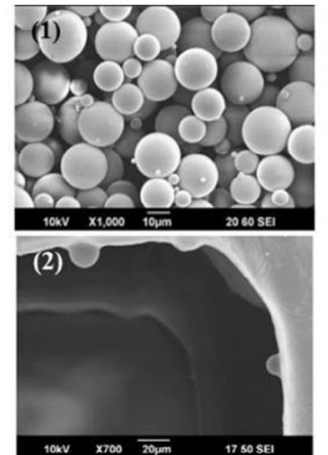

(5)

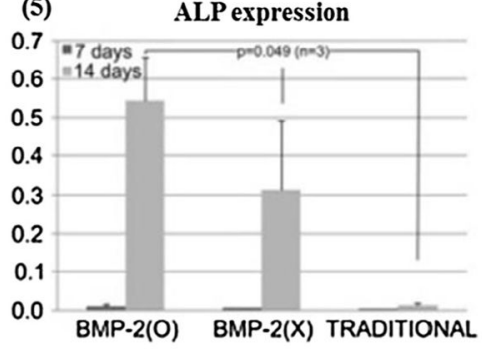

(6)

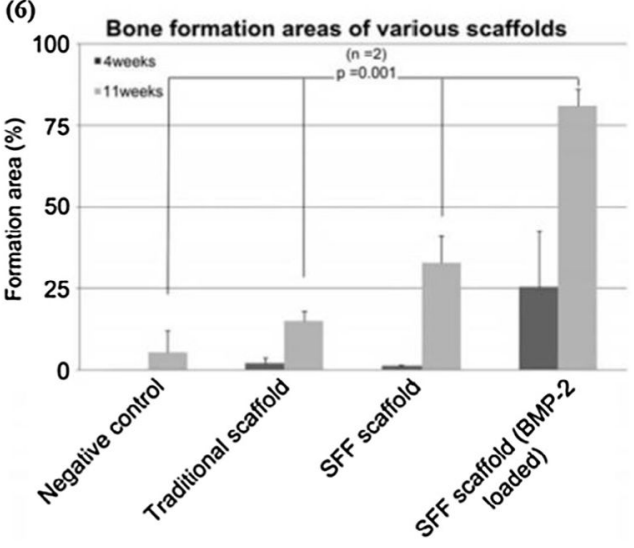

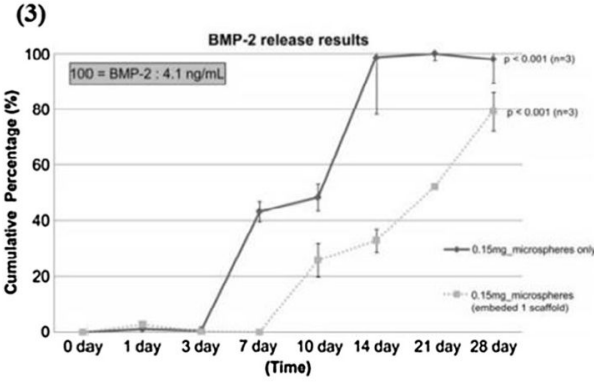

(4)
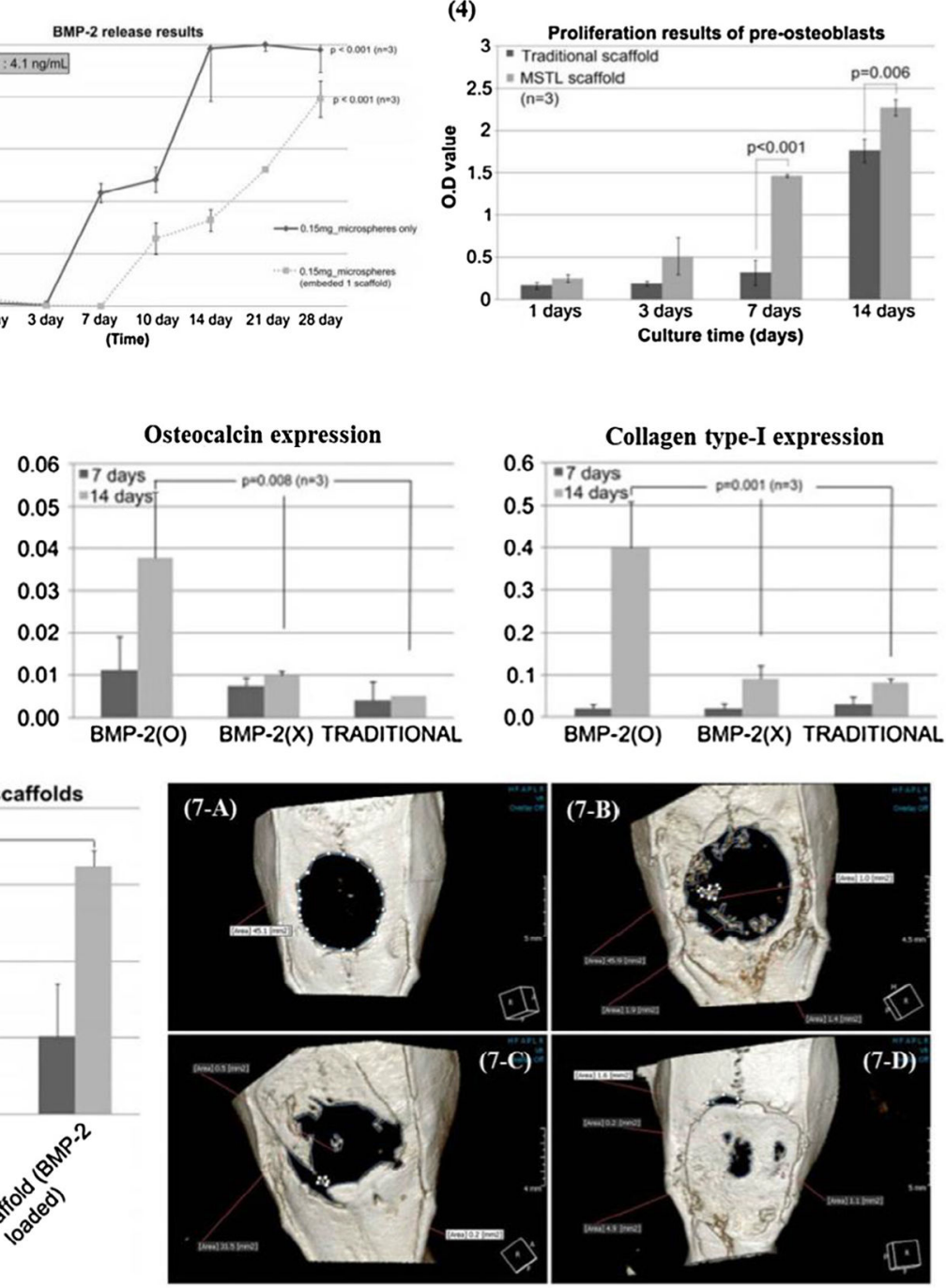

Fig. 37.

(1) Microspheres with BMP-2 (2) Scaffolds loaded with microspheres/BMP-2 (3) BMP-2 release on microsphere only and microsphere loaded scaffolds (4) Cell proliferation analysis showing the enhancement of MSTL scaffolds on pre-osteoblasts in vitro. (5) Cell differentiation evaluation by performing RT-PCR using ALP, osteocalcin and collagen type I as markers on BMP-2 loaded MSTL scaffolds, BMP-2 unloaded MSTL scaffolds and traditional scaffolds. (6) Bone formation area of different scaffolds after implanting into rat cranial bone defect model for 6 and 11 weeks. From (7-A) to (7-D), they were CT images of negative control, BMP-2-unloaded traditional scaffold, BMP-2-unloaded MSTL scaffold and BMP-2-loaded SFF scaffold after 11 weeks of in vivo study in rat cranial bone defect model. Controlled release of BMP-2 significantly improved the new bone formation [219]. 
(1)
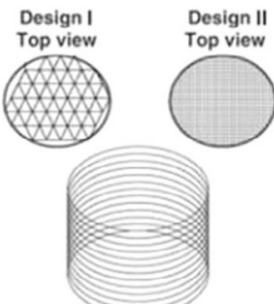

Prototype Design III

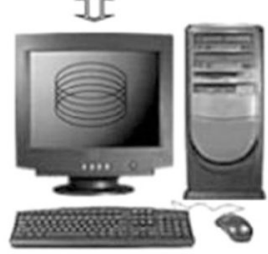

(3)

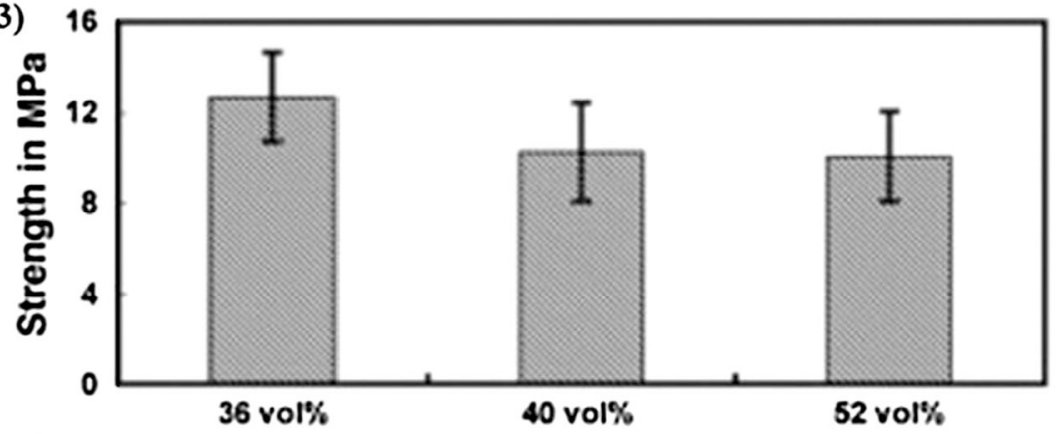

(2)

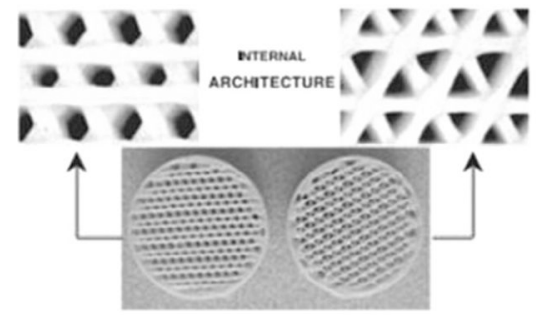

3-D Honeycomb porous PP.TCP composito scattolds
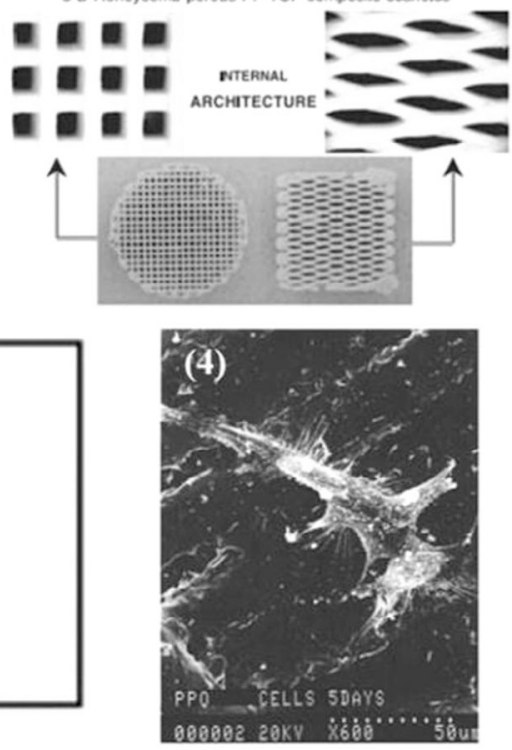

Fig. 38.

(1) The schematic the FDM process for making composite scaffolds (2) Final scaffolds with different pore designs. (3) The compressive strength of scaffolds with different porosities.

(4) The SEM image showing osteoprecussor cells adhesion [236]. 
(1)

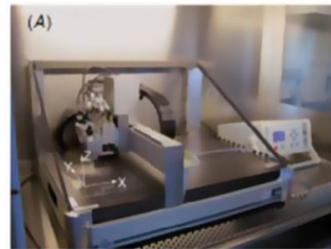

(C)
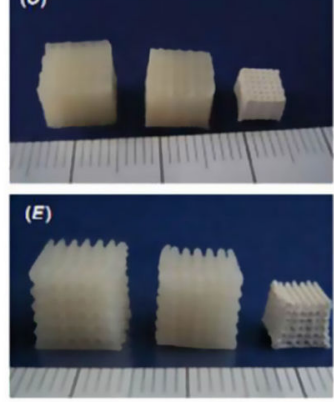
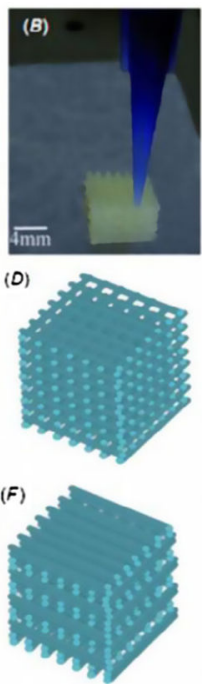
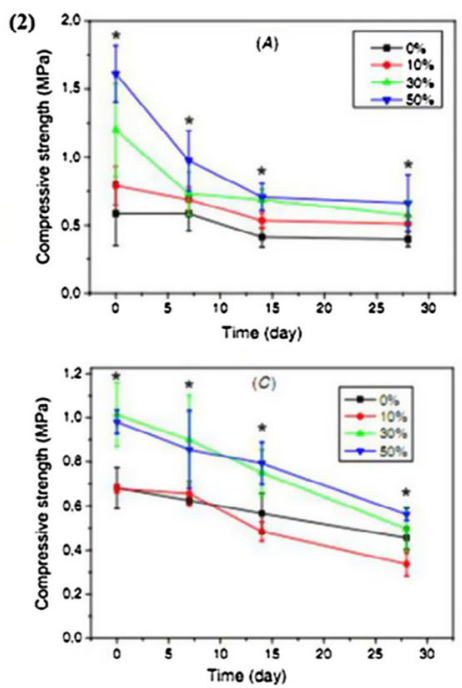
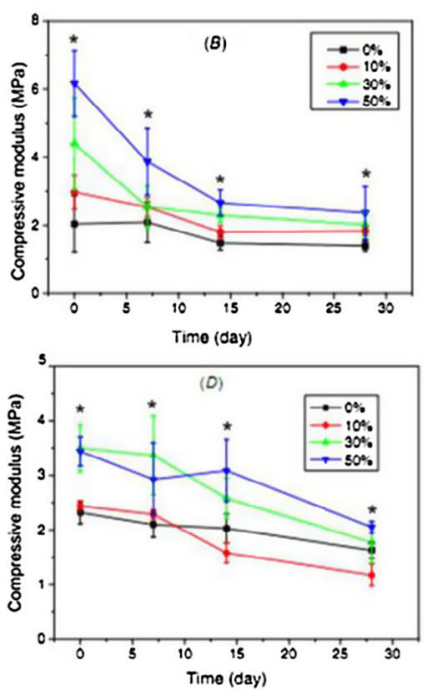
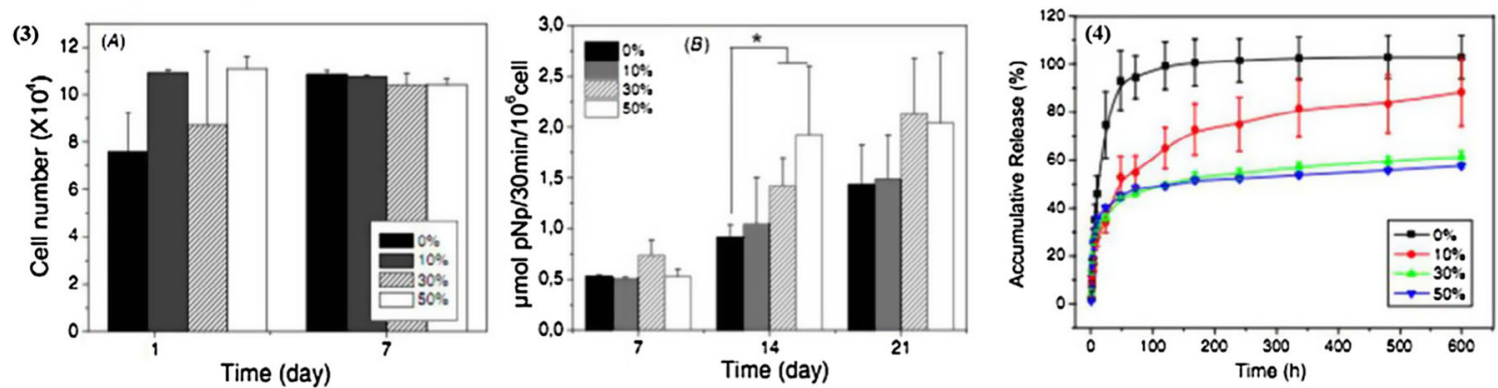

Fig. 39.

(1) The 3D-plotting system (A), the scaffold in printing (B), CAD design of XY (D) and XXYY pattern (F) and printed scaffolds (wet state, after crosslinking and dry state, from left to right) with XY (C) and XXYY pattern $(\mathrm{F})$. (2) The compressive strength and modulus of scaffolds with $0 \%, 10 \%, 30 \%$ and $50 \%$ of bioglass after certain time of SBF immersion ((AB)-XY pattern and (C-D)-XXYY pattern). (3) The human bone marrow-derived mesenchymal stem cells viability (A) and ALP activity of scaffolds with $0 \%, 10 \%, 30 \%$ and $50 \%$ of bioglass after 1 and 7 days of culturing. (4) The accumulative release of dexamethasone loaded on scaffolds with $0 \%, 10 \%, 30 \%$ and $50 \%$ of bioglass [239]. 
a

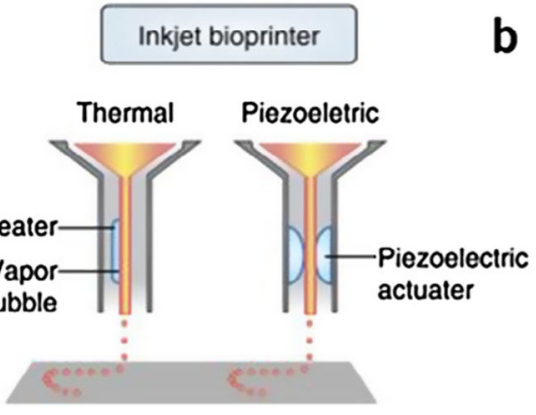

b

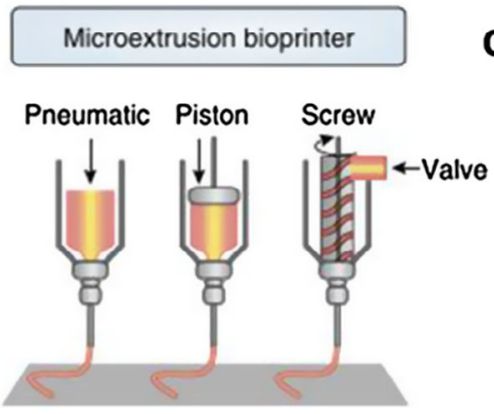

C

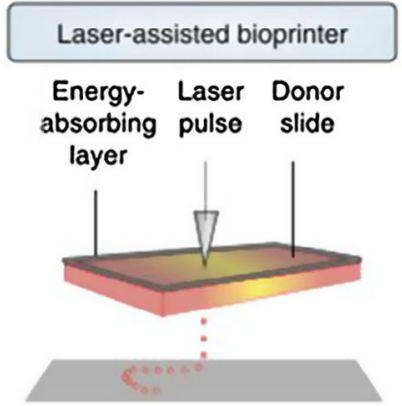

Fig. 40.

The schematics of three major bioprinters [261,262]. 

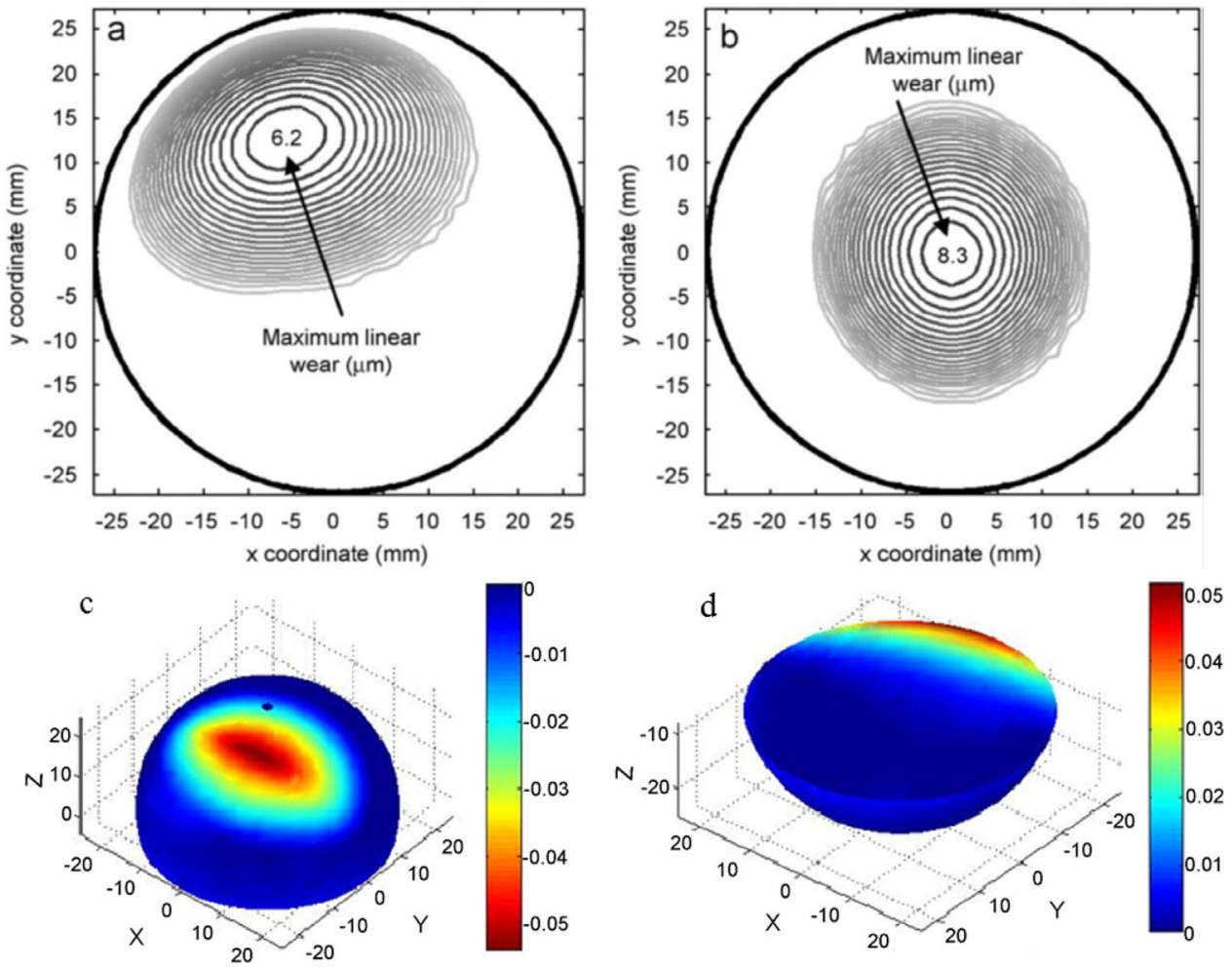

Fig. 41.

(a) Computationally predicted linear wear contours of wear geometry on (a) Head and (b) Cup for 50 million cycles as viewed in the direction that is through the center and pole of the cup surface [301]; Wear map generated by a custom-made Matlab program on retrieved ASR $^{\text {TM }}$ head and it's paired cup after 9 months. Dark blue color shows areas not worn whereas red color indicates the deepest worn areas [302]. 


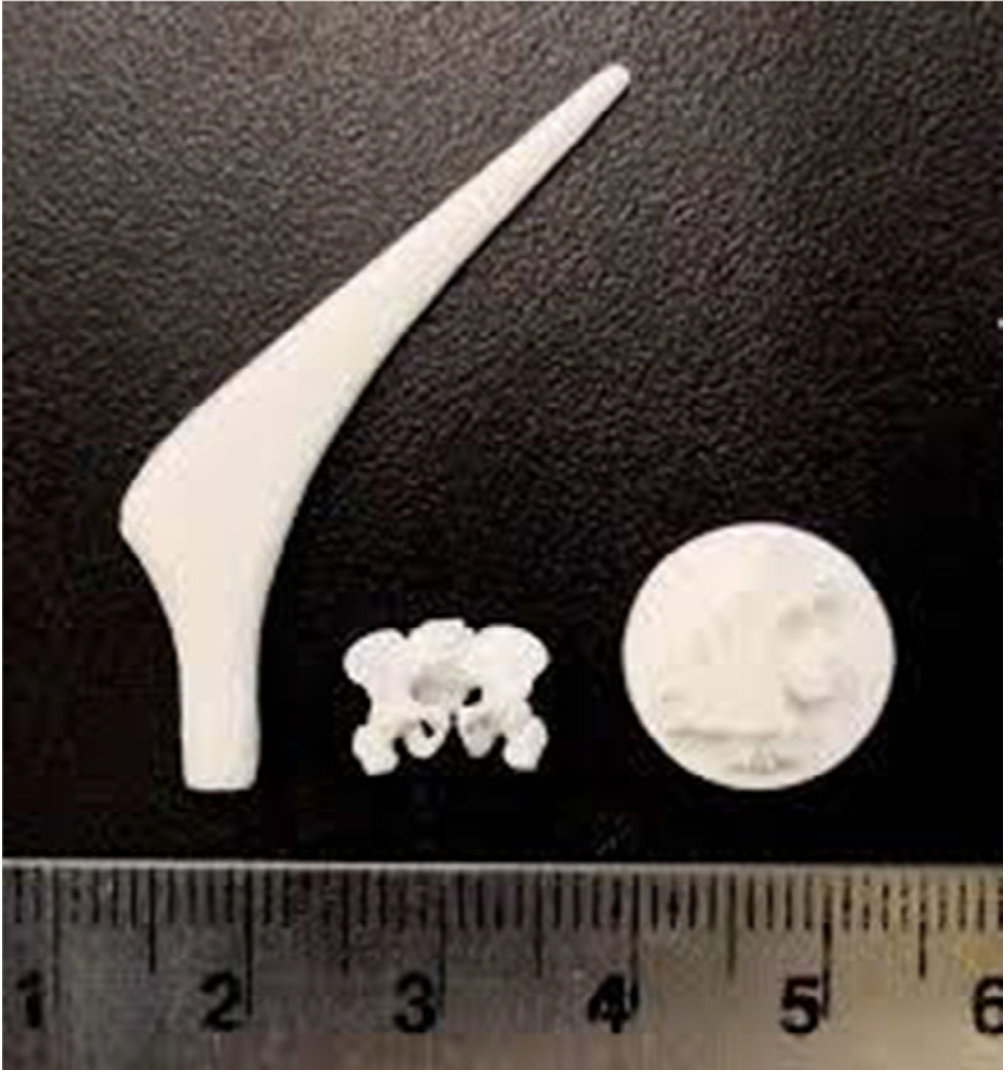

Fig. 42.

Hip stem, human pelvis and WSU logo printed by binder jetting 3D printer using TCP [94]. 

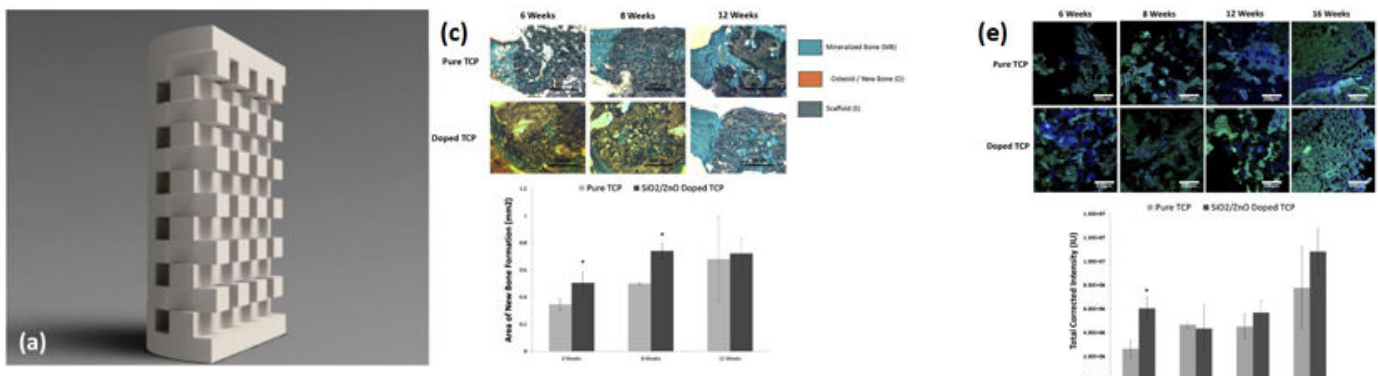

(d)
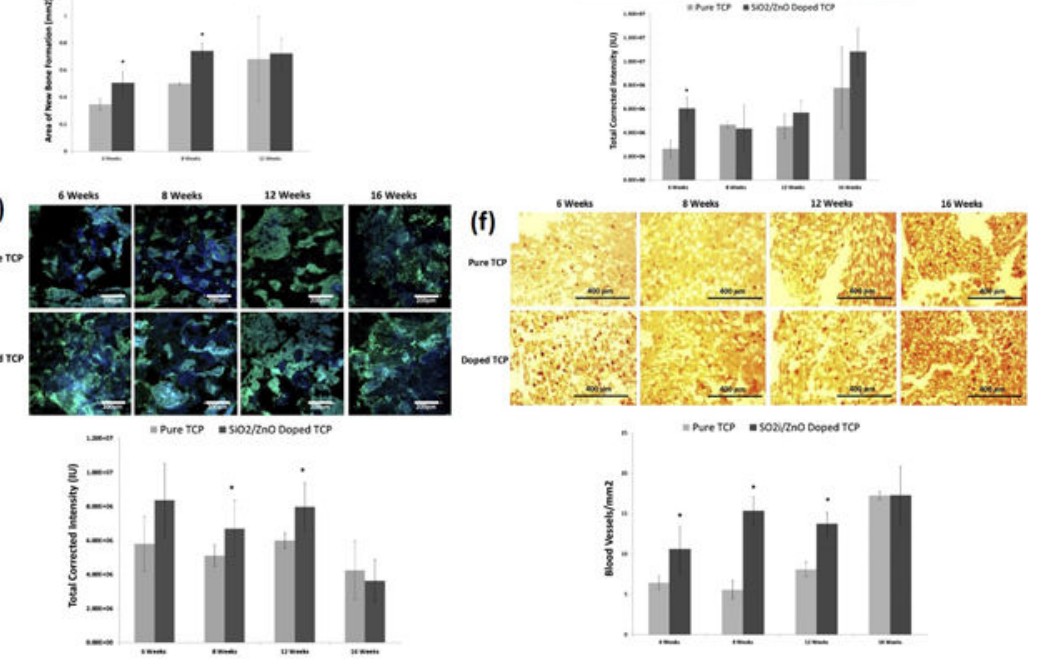

Fig. 43.

(a) CAD design of the porous scaffolds (b) Schematic of binder jetting 3D printing of porous TCP scaffolds (c) Goldner's trichrome staining of the implant section and histomorphometry of new bone formation (orange) (d) Confocal image of collagen I formation (green) and its histomorphometry (e) Confocal image of osteocalcin (green) and its histomorphometry (f) vWF staining of the implant section and histomorphometry of blood vessels (dark red spots) [264]. 


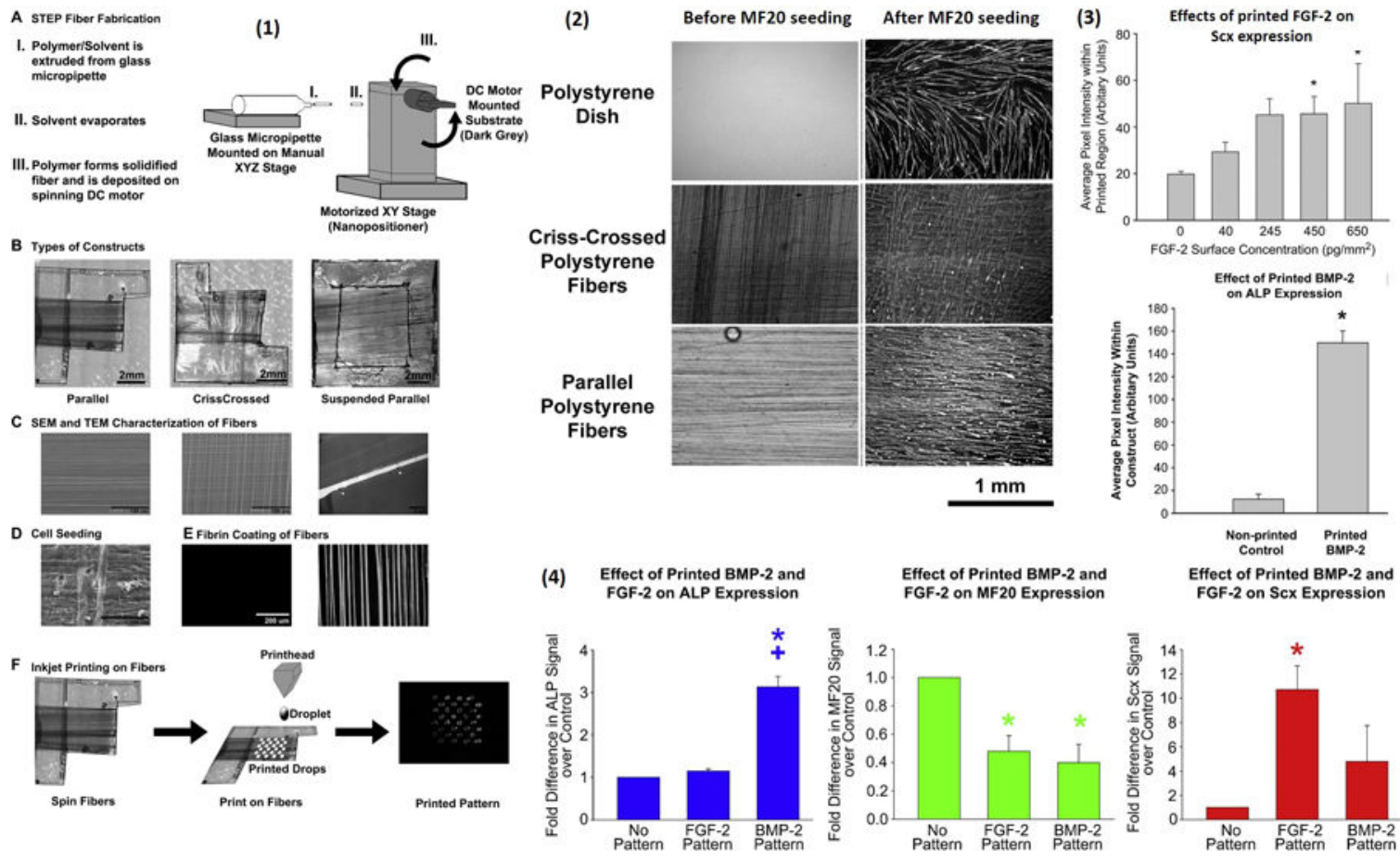

Fig. 44.

(1) Schematics of the process. (2) The oriented fiber structure and myotube alignment after the mouse myosin (MF20) seeding (3) Effects the concentration of printed FGF-2 or BMP-2 on Scx and ALP expression, respectively. (4) Effects of printed FGF-2 and BMP-2 on ALP, MF20 and Scx expressions [266]. 
(1)
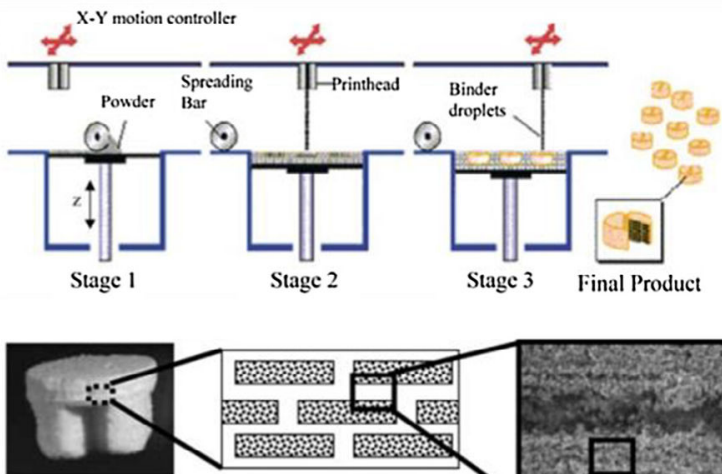

Side view

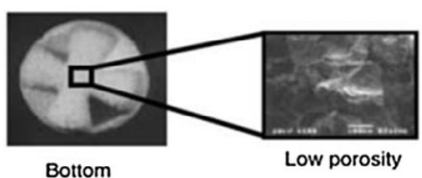

Bottom

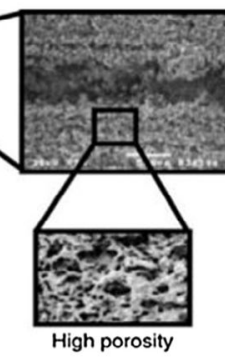

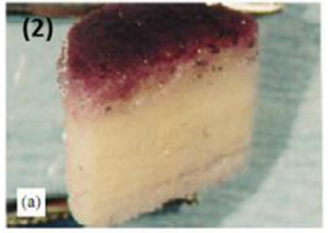
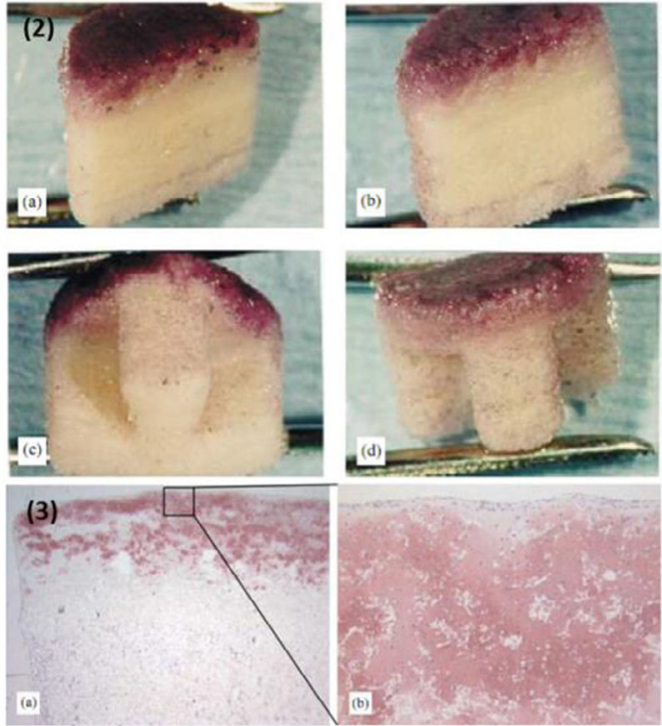

Composition of osteochondral scaffold

\begin{tabular}{lllllr}
\hline Region & $\begin{array}{l}\text { Amount of } \mathrm{NaCl} \\
(w t \%)\end{array}$ & $\begin{array}{l}\text { Size of } \mathrm{NaCl} \\
(\mu \mathrm{m})\end{array}$ & $\begin{array}{l}\text { PLGA(50:50) } \\
(\mathrm{wt} \%)\end{array}$ & $\begin{array}{l}\text { PLA } \\
(\mathrm{wt} \%)\end{array}$ & $\begin{array}{l}\text { PLGA(85:15) } \\
(w t \%)\end{array}$ \\
\hline Cartilage & 90 & $106-150$ & 5 & 5 & - \\
Transition 1 & 85 & $106-150$ & 5 & 5 & - \\
Transition 2 & 75 & $106-150$ & 5 & 5 & 15 \\
Transition 3 & 65 & $106-150$ & 2.5 & 2.5 & 30 \\
Bone & 55 & $125-150$ & - & - & 33.75
\end{tabular}

Tensile and compressive testing data. Averages and standard deviations for $n=3$ or 4

\begin{tabular}{|c|c|c|c|c|c|c|}
\hline \multicolumn{3}{|c|}{ Composition } & \multicolumn{2}{|l|}{ Tensile data } & \multicolumn{2}{|c|}{ Compressive data } \\
\hline $\begin{array}{l}\mathrm{NaCl} \\
(\%)\end{array}$ & $\begin{array}{l}\text { TCP } \\
(\%)\end{array}$ & $\begin{array}{l}\text { L.PLGA } \\
(\%)\end{array}$ & $\begin{array}{l}\text { Tensile strength } \\
(\mathrm{MPa})\end{array}$ & $\begin{array}{l}\text { Elastic modulus } \\
\text { (MPa) }\end{array}$ & $\begin{array}{l}\text { Yield strength } \\
\text { (MPa) }\end{array}$ & $\begin{array}{l}\text { Elastic modulus } \\
(\mathrm{MPa})\end{array}$ \\
\hline 25 & 25 & 50 & $5.7 \pm 1.0$ & $200 \pm 57$ & $13.5 \pm 0.3$ & $233 \pm 26$ \\
\hline 35 & 15 & 50 & $5.5 \pm 0.8$ & $233 \div 27$ & $13.7 \pm 0.8$ & $450 \pm 79$ \\
\hline 35 & 21.7 & 43.3 & $3.3 \pm 0.4$ & $180 \pm 14$ & $6.5 \pm 0.2$ & $184 \pm 12$ \\
\hline 40 & 15 & 45 & $4.0 \pm 0.5$ & $183 \pm 35$ & $7.0 \pm 0.9$ & $180 \pm 50$ \\
\hline 55 & 11.25 & 33.75 & $1.6 \pm 0.2$ & $83 \pm 18$ & $2.5 \pm 0.1$ & $54 \pm 17$ \\
\hline \multicolumn{3}{|c|}{ Cancellous human bone (fresh) [52] } & $\sim 8$ & $\sim \overline{700}-1000$ & $10-\overline{20}$ & \\
\hline
\end{tabular}

Fig. 45.

(1) Top one is the schematic of the machine used for making scaffolds. Bottom one shows the final scaffolds and the microstructure of different regions. (2) Cross-section and outer images of MTT stained osteochondral scaffolds after $24 \mathrm{~h}$ of a top seeding (a, c) and rotational seeding $(b, d)$ methods using ovine articular chondrocytes from the femoral condyle. Two tables contain the compositional information and mechanical property of the composite scaffold [271]. 
(1) a

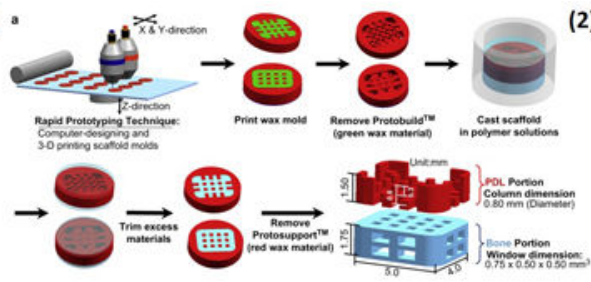

b

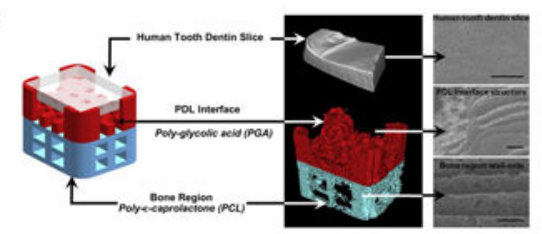

(2) ${ }^{a}$
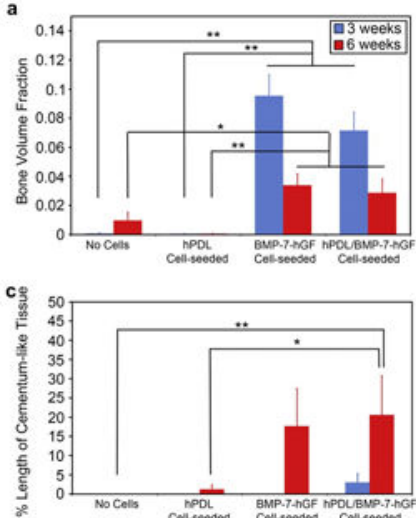
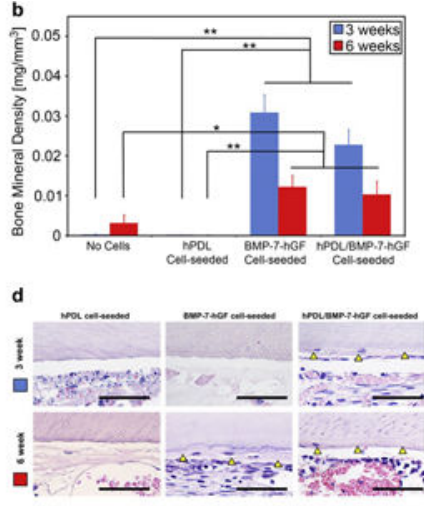

Fig. 46.

(1-a) The schematic of the manufacture for the tooth-ligament complex. (1-b) The final assembly structure and microstructure of different regions. (2a-c) Bone volume fraction, bone mineral density and length of cementum- like tissue after implantation for 3 and 6 weeks. (2-d) H\&E staining images showing cementum-like tissue formation on the dentin surface at 3 weeks for hPDL/BMP-7-hGF cell-seeded samples and at 6 weeks for BMP-7hGF cell-seeded and hPDL/BMP-7-hGF cell-seeded samples [280]. 
(1)
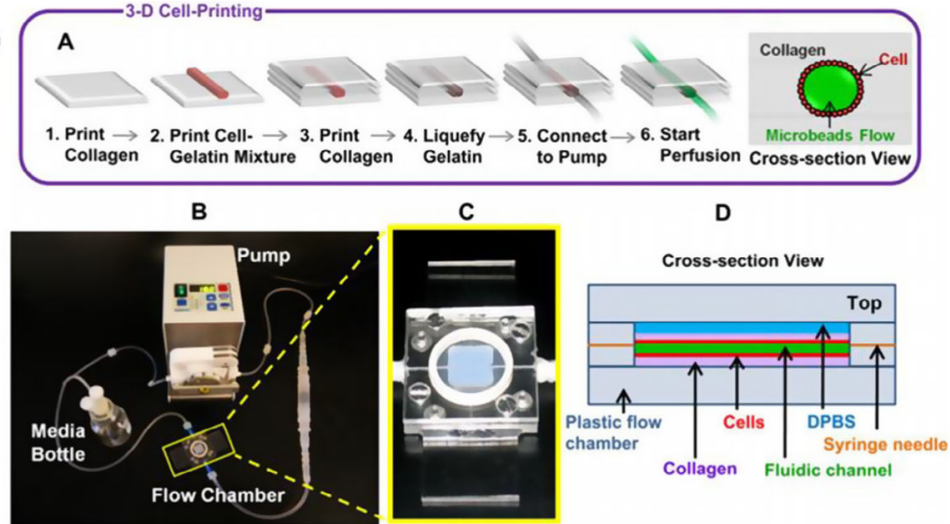

(2) A

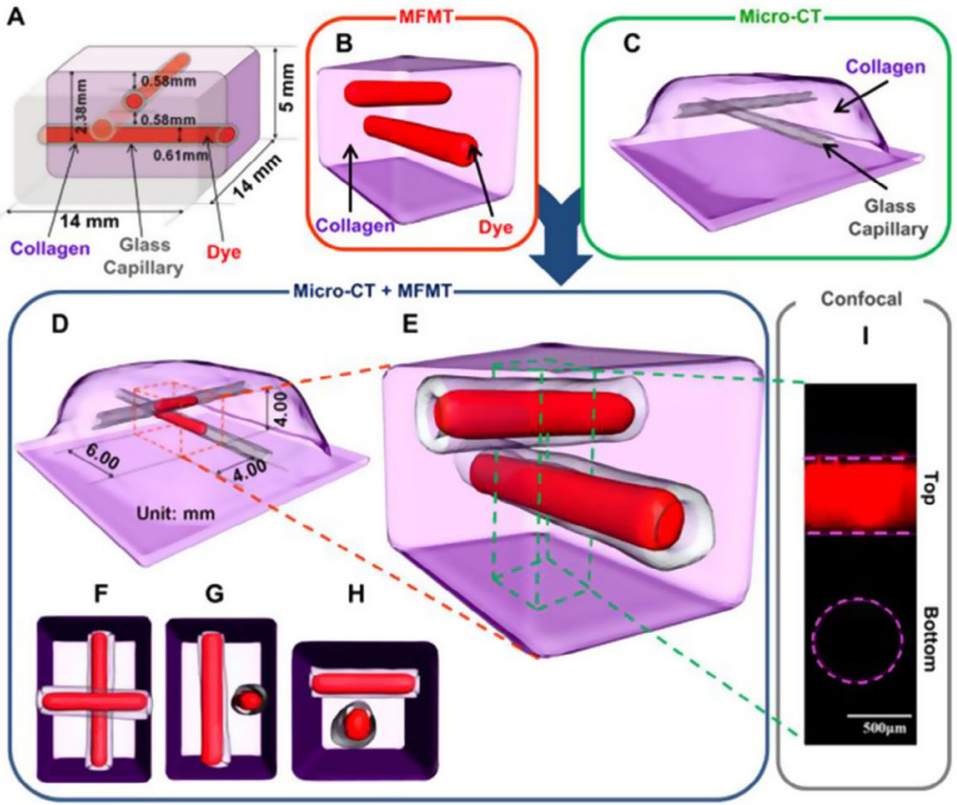

Fig. 47.

(1-A) The schematic of the manufacture of vascular constructs. The perfusion setup (1-B) with the perfusion chamber (1-C) and cross-section view under the perfusion process (1-D). (2-A) The designed vascular construct structure. MFMT image (2-B), micro-CT image (2-C) and combined images (2-D-H). (2-I) The confocal image. The pink dashed lines showed the top blood vessel, and the pink dashed circle showed the deeper inclusion vascular area located by micro-CT [287]. 

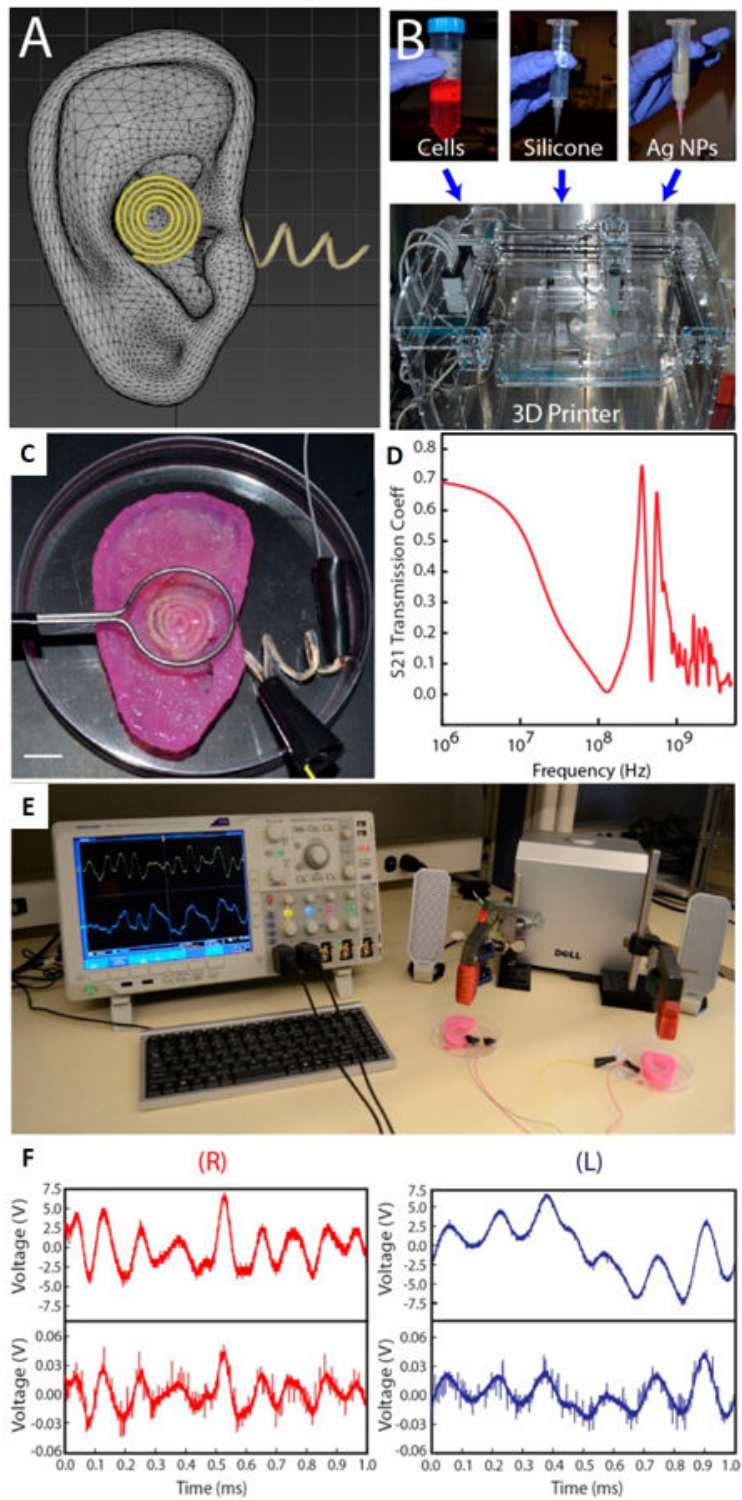

Fig. 48.

(A) The design of the bionic ear. (B) The printing device with different printing inks. (C) Image of the experimental setup used to characterize the bionic ear. The ear is exposed to a signal from a transmitting loop antenna. The output signal is collected via connections to two electrodes on the cochlea. Scale bar is $1 \mathrm{~cm}$. (D) Response of the bionic ear to radio frequencies (E) Photograph of complementary bionic ears listening to stereophonic audio music (F) Transmitted (top) and received (bottom) audio signals of the right (R) and left (L) bionic ears [294]. 


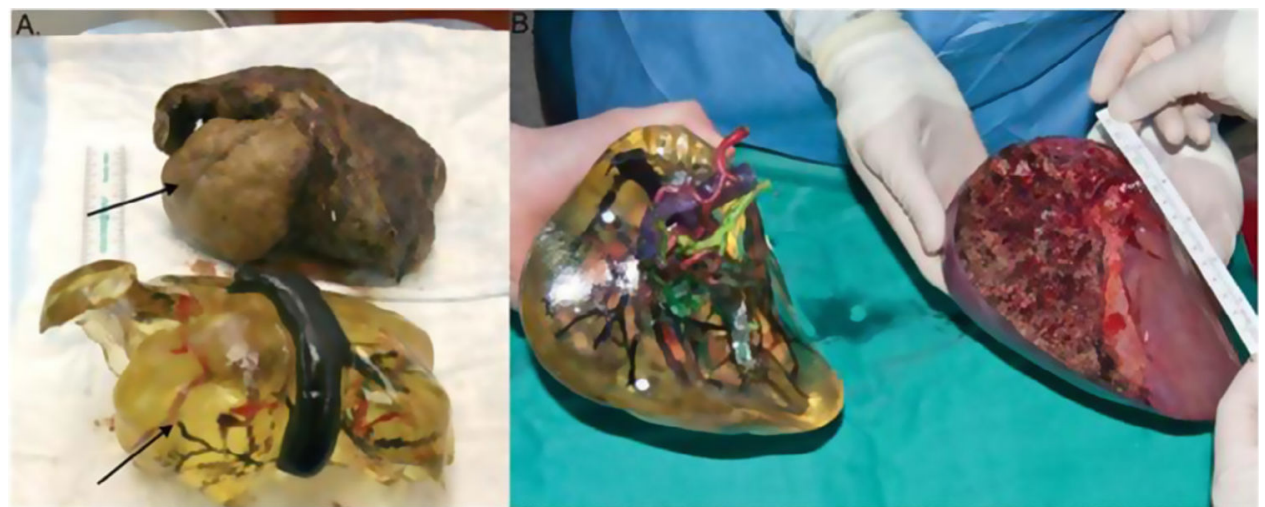

Fig. 49.

(A) Actual liver of a recipient and 3D-printed liver. The arrows indicates a regenerative nodule in both and 3D-printed liver and the native liver. (B) Preoperatively 3D-printed right lobe and actual right lobe of a donor [297]. 


\section{Table 1}

Particle size and shape requirements for dry/wet powder binder jetting.

\begin{tabular}{lll}
\hline & Dry powder binder jetting & Wet powder binder jetting \\
\hline Preferred particle size & $>20 \mu \mathrm{m}[2-5]$ & $<5 \mu \mathrm{m} \mathrm{[2]}$ \\
Preferred particle shape & Spherical powders [6,7] & Faceted and anisotropic powders [7] \\
\hline
\end{tabular}


Table 2

Binder categories with their available materials, advantages and disadvantages.

\begin{tabular}{|c|c|c|c|c|}
\hline $\begin{array}{l}\text { Binder } \\
\text { categories }\end{array}$ & Available materials & Advant: & & Disadvantages \\
\hline Organic binder & $\begin{array}{l}\text { Butyral resins [7] } \\
\text { Polymeric resins [3] } \\
\text { Polyvinyls [15,16] } \\
\text { Carbohydrates [17] } \\
\text { Polycarbosilazane [7] } \\
\text { Polysiloxanes [18] } \\
\text { Aluminum amides [19] } \\
\text { Pyrrolidone [20] }\end{array}$ & 2 & $\begin{array}{l}\text { It can be used almost for all powder } \\
\text { materials. } \\
\text { It can be easily removed with little } \\
\text { residue. }\end{array}$ & It is easy to clog the print head \\
\hline $\begin{array}{l}\text { Inorganic } \\
\text { binder }\end{array}$ & $\begin{array}{l}\text { Colloidal silica [12] } \\
\text { Aluminum nitrate [21] } \\
\text { Alumina [22] }\end{array}$ & \multicolumn{2}{|c|}{$\begin{array}{l}\text { The whole bed can be heated to bind parts within } \\
\text { the bed }\end{array}$} & $\begin{array}{l}\text { It may not react with powder } \\
\text { immediately after deposition }\end{array}$ \\
\hline In-bed binder & $\begin{array}{l}\text { Maltodextrin [23] } \\
\text { Sucrose }[24] \\
\text { Plasters }[14] \\
\text { Cements }[3,13]\end{array}$ & 2 & $\begin{array}{l}\text { It is not specific to certain powder } \\
\text { and can be totally removed by high } \\
\text { temperature } \\
\text { Deposited liquid is simple and } \\
\text { reliable }\end{array}$ & $\begin{array}{l}\text { The optimization steps are } \\
\text { sometimes complicated }\end{array}$ \\
\hline $\begin{array}{l}\text { Acid-base } \\
\text { binder }\end{array}$ & $\begin{array}{l}10 \mathrm{v} / \mathrm{v} \% \text { phosphoric acid and citric } \\
\text { acid [25] } \\
\text { Polyvinyl pyrrolidone [26] }\end{array}$ & \multicolumn{2}{|c|}{$\begin{array}{l}\text { Strong binding forms with barely any residues } \\
\text { after thermal treatment }\end{array}$} & $\begin{array}{l}\text { It is limited to only a small } \\
\text { range of powders }\end{array}$ \\
\hline $\begin{array}{l}\text { Metal salts } \\
\text { binder }\end{array}$ & Silver nitrate [27] & \multicolumn{2}{|c|}{$\begin{array}{l}\text { Multiple binding pathways, such as salt } \\
\text { recrystallization, salt reduction and salt } \\
\text { displacement reaction, can be used }\end{array}$} & $\begin{array}{l}\text { In salt reduction pathway, bulk } \\
\text { material must be resistant to the } \\
\text { heat reduction }\end{array}$ \\
\hline Solvent binder & Chloroform $[28,29]$ & \multicolumn{2}{|c|}{ Part purity is high } & $\begin{array}{l}\text { It is a method normally for } \\
\text { polymers }\end{array}$ \\
\hline $\begin{array}{l}\text { Phase-changing } \\
\text { binder }\end{array}$ & 2-Methylpropane-2-OL [30] & \multicolumn{2}{|c|}{ It can be used for most powder } & $\begin{array}{l}\text { There is a limit for post print } \\
\text { heating }\end{array}$ \\
\hline $\begin{array}{l}\text { Sintering } \\
\text { inhibition } \\
\text { binder }\end{array}$ & $\begin{array}{l}\text { heat-isolating materials, chemical } \\
\text { oxidizers, sintering inhibitors and } \\
\text { heat-reflective materials [31] }\end{array}$ & \multicolumn{2}{|c|}{ It only needs jetting at the part boundary } & $\begin{array}{l}\text { Excess powder will } \\
\text { contaminate the part }\end{array}$ \\
\hline
\end{tabular}




\section{Table 3}

Different print heads and their advantages and disadvantages.

\begin{tabular}{llll}
\hline Type of print heads & Subcategories & Advantages & Disadvantages \\
\hline $\begin{array}{l}\text { Drop-on-demand (DoD) } \\
\text { print heads }\end{array}$ & Piezoelectric heads & It is easy to control ink development & $\begin{array}{l}\text { Rheological property of the ink must } \\
\text { be controlled carefully }\end{array}$ \\
& Thermal inkjet heads & $\begin{array}{l}\text { No solid material is deposited, which prevents } \\
\text { from clogging the head [37] }\end{array}$ & $\begin{array}{l}\text { The dissolution of the vaporized } \\
\text { liquid must be quick }\end{array}$ \\
$\begin{array}{l}\text { Continuous-jet (CJ) print } \\
\text { heads }\end{array}$ & None & $\begin{array}{l}\text { It has options of proportional deflection, higher } \\
\text { printing rate, better surface finish and slower } \\
\text { traverse speed than DoD [36,38] }\end{array}$ & $\begin{array}{l}\text { The ink should be inductively } \\
\text { chargeable }\end{array}$ \\
\hline
\end{tabular}




\section{Table 4}

Different laser based powder bed fusions and their starting manufactures.

\begin{tabular}{ll}
\hline Laser based PBF & Starting manufacturers \\
\hline Selective laser sintering (SLS) & EOS (Krailling, Germany) \\
Selective laser melting (SLM) & SLM Solutions GmbH (Lübeck, Germany) and Über ReaLizer (Borchen, Germany) \\
Direct metal laser sintering (DMLS) & EOS (Krailling, Germany) \\
Laser curing & CONCEPTLASER (Lichtenfels, Germany) \\
\hline
\end{tabular}


Table 5

Differences of the feature between SLM and EBM [74].

\begin{tabular}{lll}
\hline & SLM EBM & SLM EBM \\
\hline Power source & One or more fiber lasers of 200-1000 W & High power Electron beam of $3000 \mathrm{~W}$ \\
Build chamber environment & Argon or Nitrogen & Vacuum/He bleed \\
Method of powder preheating & Platform heating & Preheat scanning \\
Powder preheating temperature $\left({ }^{\circ} \mathrm{C}\right)$ & $100-200$ & $700-900$ \\
Maximum available build volume $(\mathrm{mm})$ & $500 \times 350 \times 300$ & $350 \times 380(\varnothing \mathrm{xH})$ \\
Maximum build rate $\left(\mathrm{cm}^{3} / \mathrm{h}\right)$ & $20-35$ & 80 \\
Layer thickness $(\mu \mathrm{m})$ & $20-100$ & $50-200$ \\
Melt pool size $(\mathrm{mm})$ & $0.1-0.5$ & $0.2-1.2$ \\
Surface finish $(\mathrm{Ra})$ & $4-11$ & $25-35$ \\
Geometric tolerance $(\mathrm{mm})$ & $\pm 0.05-0.1$ & \pm 0.2 \\
Minimum feature size $(\mu \mathrm{m})$ & $40-200$ & 100 \\
\hline
\end{tabular}




\section{Table 6}

Photo-reactive materials and their photoinitiators.

\begin{tabular}{|c|c|c|c|}
\hline Categories & Photo-reactive polymers & Photoinitiators & References \\
\hline \multirow[t]{6}{*}{ Acrylates } & Di-ethoxylated bisphenol A dimethacrylate or diacryl 101 & $\begin{array}{l}\text { 2,2-Dimethoxy-2-phenylacetophenone or } \\
\text { DMPA }\end{array}$ & {$[81]$} \\
\hline & 1,6 Hexanediol diacrylate (HDDA) & 1-Hydroxy-cyclohexyl phenyl ketone & {$[82]$} \\
\hline & \multirow[t]{2}{*}{ Acrylamide } & Phosphine oxide and ketone derivative & {$[83]$} \\
\hline & & Ammonium persulfate & {$[84,85]$} \\
\hline & Acrylic and silicon acrylate & $\begin{array}{l}\text { 1-Hydroxy-cyclohexyl-phenyl-khetone } \\
\text { Irgacure } 184\end{array}$ & {$[86,87]$} \\
\hline & $\begin{array}{l}2,2^{\prime} \text {-bis [4-(methacryloxyethoxy)phenyl]propane (Diacryl } \\
101 \text { ) and HDDA }\end{array}$ & 2,2'-Dimethoxy-2-phenylaceto-phenone & {$[88,89]$} \\
\hline \multirow[t]{3}{*}{ Epoxies } & SL5180 resin & Sulfonium salt & {$[90]$} \\
\hline & 3,4-epoxycyclohexylmethyl-3,4-epoxycyclohexylcarboxylate & $\begin{array}{l}\text { 2,2'-Dimethoxy-2-phenylaceto-phenone } \\
\text { (DMPA) }\end{array}$ & {$[88,89]$} \\
\hline & SL5170 resin & Sulfonium salt & [91] \\
\hline $\begin{array}{l}\text { Combined } \\
\text { photo-reactive } \\
\text { materials }\end{array}$ & $\begin{array}{l}\text { 4-Epoxycyclohexylmethyl-3,4-epoxycyclohexylcarboxylate } \\
\text { and trimethylolpropane triacrylate (SOMOS 6100) }\end{array}$ & $\begin{array}{l}\text { 2,2'-Dimethoxy-2-phenylaceto-phenone } \\
\text { (DMPA) }\end{array}$ & {$[88,89]$} \\
\hline
\end{tabular}

Prog Mater Sci. Author manuscript; available in PMC 2019 August 12. 
Table 7

The dissolution property of several important calcium phosphate materials with their $\mathrm{Ca}$ to $\mathrm{P}$ ratio $[95,97]$.

\begin{tabular}{|c|c|c|c|c|c|c|}
\hline Chemical name & $\begin{array}{l}\text { Usual } \\
\text { symbol }\end{array}$ & Chemical formulation & Mineral name & $\begin{array}{l}\text { Atomic ratio } \\
\mathrm{Ca} / \mathbf{P}\end{array}$ & $\begin{array}{l}\text { Space } \\
\text { group }\end{array}$ & Solubility product \\
\hline Monocalcium phosphate & MCP & $\mathrm{Ca}\left(\mathrm{H}_{2} \mathrm{PO}_{4}\right)_{2} \cdot \mathrm{H}_{2} \mathrm{O}$ & - & 0.5 & - & $1.0 \times 10^{-3}$ \\
\hline $\begin{array}{l}\text { Dicalcium phosphate } \\
\text { dihydrate }\end{array}$ & DCPD & $\mathrm{CaHPO}_{4} \cdot 2 \mathrm{H}_{2} \mathrm{O}$ & Brushite & 1.00 & $2 / m$ & $1.87 \times 10^{-7}$ \\
\hline Dicalcium phosphate & $\mathrm{DCP}$ & $\mathrm{CaHPO}_{4}$ & Monetite & 1.00 & $P 1$ & $1.26 \times 10^{-7}$ \\
\hline Octocalcium phosphate & $\mathrm{OCP}$ & $\mathrm{Ca}_{8} \mathrm{H}_{2}\left(\mathrm{PO}_{4}\right)_{6} \cdot 5 \mathrm{H}_{2} \mathrm{O}$ & - & 1.33 & - & $5.01 \times 10^{-15}$ \\
\hline Tricalcium phosphate & TCP & $\mathrm{Ca}_{3}\left(\mathrm{PO}_{4}\right)_{2}$ & - & 1.50 & $R 3 c$ & $2.83 \times 10^{-30}$ \\
\hline Hydroxyapatite & HAP & $\mathrm{Ca}_{10}\left(\mathrm{PO}_{4}\right)_{6} \cdot(\mathrm{OH})_{2}$ & Hydroxyapatite & 1.67 & $P 6_{3} / m$ & $2.35 \times 10^{-59}$ \\
\hline Tetra-calcium phosphate & TTCP & $\mathrm{Ca}_{4} \mathrm{O}\left(\mathrm{PO}_{4}\right)_{2}$ & - & 2.00 & $P 2_{1}$ & - \\
\hline
\end{tabular}




\section{Table 8}

The AM processings of ceramic/polymer composites with their rationales.

\begin{tabular}{|c|c|c|c|c|}
\hline Ceramic & Polymer & AM method & Rationale & Reference \\
\hline \multirow[t]{2}{*}{ HA } & \multirow[t]{2}{*}{ PCL } & $\begin{array}{l}\text { Material } \\
\text { extrusion (wet- } \\
\text { spinning) }\end{array}$ & $\begin{array}{l}\text { PCL has excellent biocompatibility and slow degradation, while } \\
\text { it can lead inflammatory reaction and the loss of mechanical } \\
\text { strength caused by the degradation. HA can enhance the } \\
\text { bioactivity and mechanical properties of PCL and reduce the acid } \\
\text { environment caused by the PCL degradation }\end{array}$ & {$[246]$} \\
\hline & & SLS & $\begin{array}{l}\text { PCL has excellent thermal stability for SLS. HA can further } \\
\text { improve the biocompatibility and bioactivity of PCL }\end{array}$ & {$[247,248]$} \\
\hline Nano-HA & PDLLA & SLA & $\begin{array}{l}\text { PDLLA is appropriate for SLA process, but its brittleness limits } \\
\text { its application. Nanometer HA is able to improve biological and } \\
\text { mechanical properties of the composite scaffolds }\end{array}$ & {$[243]$} \\
\hline \multirow[t]{5}{*}{$\begin{array}{l}\text { TCP } \\
\text { TCP }\end{array}$} & PLGA/PCL & $\begin{array}{l}\text { Material } \\
\text { extrusion } \\
\text { (multihead } \\
\text { deposition) }\end{array}$ & $\begin{array}{l}\text { PLGA has positive effects on tissue reconstruction, but it can't } \\
\text { maintain the shape because of its bad mechanical property and } \\
\text { rapid degradation. TCP has excellent biocompatibility, } \\
\text { osteoconductivity and can improve the mechanical property of } \\
\text { PLGA. PCL is able to improve the processibility and toughness } \\
\text { of the composite }\end{array}$ & [249] \\
\hline & Polypropylene (PP) & FDM & $\begin{array}{l}\text { PP has high flex life, great stress cracking resistance as well as } \\
\text { good processing ability by FDM. TCP is an accepted bone } \\
\text { substitute material. }\end{array}$ & {$[236]$} \\
\hline & PCL & FDM & $\begin{array}{l}\text { PCL has a low melting point of } 60{ }^{\circ} \mathrm{C} \text { and high deposition } \\
\text { temperature of } 350{ }^{\circ} \mathrm{C} \text {, which is excellent for FDM process. TCP } \\
\text { is bioactive, osteoconductive and shows minimal immunologic } \\
\text { response }\end{array}$ & {$[250-252]$} \\
\hline & PDLLA & SLM & $\begin{array}{l}\text { PDLLA has low melting point to glue TCP within the PDLLA } \\
\text { matrix. In addition, incorporating TCP can reduce the acid } \\
\text { environment caused by PDLLA degradation. }\end{array}$ & {$[242]$} \\
\hline & PLLA & $\begin{array}{l}\text { Material } \\
\text { extrusion (low- } \\
\text { temperature } \\
\text { deposition) }\end{array}$ & $\begin{array}{l}\text { The PLLA is needed for the low temperature process. The } \\
\text { addition of TCP can improve its mechanical and biological } \\
\text { properties }\end{array}$ & {$[253]$} \\
\hline HA/ТCP & Agarose & $\begin{array}{l}\text { SLA (Negative } \\
\text { SLA mold } \\
\text { elimination) }\end{array}$ & $\begin{array}{l}\text { Agarose is a biocompatible hydrogel. HA/TCP has excellent } \\
\text { biocompatibility, bioactivity and osteoinductivity for bone } \\
\text { reconstruction }\end{array}$ & {$[254]$} \\
\hline \multirow[t]{2}{*}{ Bioglass } & PCL & SLA FDM & $\begin{array}{l}\text { Bioglass can interact with bone tissues and form strong bonding } \\
\text { and the released ions can induce angiogenesis, but it has bad } \\
\text { processibility and roughness. In addition, certain bioglasses have } \\
\text { antimicrobial effects. PCL has excellent processing ability and } \\
\text { biocompatibility as well }\end{array}$ & {$[237,244]$} \\
\hline & PLA/PEG & $\begin{array}{l}\text { Material } \\
\text { extrusion } \\
\text { (nozzle- } \\
\text { deposition) }\end{array}$ & $\begin{array}{l}\text { PLA is a FDA approved biodegradable polymer. Bioglass has } \\
\text { controlled biodegrafability and excellent bioactivity in vitro and } \\
\text { in vivo. PEG works as plasticizer for assisting the processing }\end{array}$ & {$[213]$} \\
\hline $\begin{array}{l}\text { Mesoporous } \\
\text { bioactive glass } \\
\text { (MBG) }\end{array}$ & Alginate & $\begin{array}{l}\text { Material } \\
\text { extrusion (3D } \\
\text { plotting) }\end{array}$ & $\begin{array}{l}\text { MBG has mesopore-channels with high orders leading its } \\
\text { excellent bioactivity. Alginate is featured with excellent } \\
\text { biocompatibility, biodegradation as well as crosslinking ability } \\
\text { under mild conditions }\end{array}$ & [239] \\
\hline \multirow[t]{2}{*}{$\begin{array}{l}\text { Calcium } \\
\text { phosphate }\end{array}$} & Collagen & Binder jetting & $\begin{array}{l}\text { Calcium phosphate can bound with binder through dissolution- } \\
\text { precipitation reaction. Incorporating collagen can improve } \\
\text { biomechanical property, osteoconductivity and osteoinductivity }\end{array}$ & {$[245]$} \\
\hline & PCL & $\begin{array}{l}\text { Negative } \\
\text { material } \\
\text { extrusion for } \\
\text { mold followed } \\
\text { by injection } \\
\text { molding }\end{array}$ & $\begin{array}{l}\text { PCL has excellent biodegradability and innate rigidity for making } \\
\text { scaffolds. Calcium phosphate is the major inorganic component } \\
\text { of the bone }\end{array}$ & {$[255]$} \\
\hline $\begin{array}{l}\text { Carbonated } \\
\text { hydroxyapatite }\end{array}$ & PLLA & SLS & $\begin{array}{l}\text { Both PLLA and PHBV have excellent biodegradability and } \\
\text { biocompatibility with blood and tissue. In addition, PLLA owns } \\
\text { excellent solubility and processibility in organic solvent. Nano }\end{array}$ & {$[241]$} \\
\hline
\end{tabular}




\begin{tabular}{|c|c|c|c|c|}
\hline Ceramic & Polymer & AM method & Rationale & Reference \\
\hline $\begin{array}{l}\text { Nano calcium } \\
\text { phosphate }\end{array}$ & $\begin{array}{l}\text { Poly } \\
\text { (hydroxybutyrate- } \\
\text { co-hydroxyvalerate) } \\
\text { (PHBV) }\end{array}$ & & $\begin{array}{l}\text { calcium phosphates have high surface area with biomimetic } \\
\text { similarity to the natural bone }\end{array}$ & \\
\hline
\end{tabular}




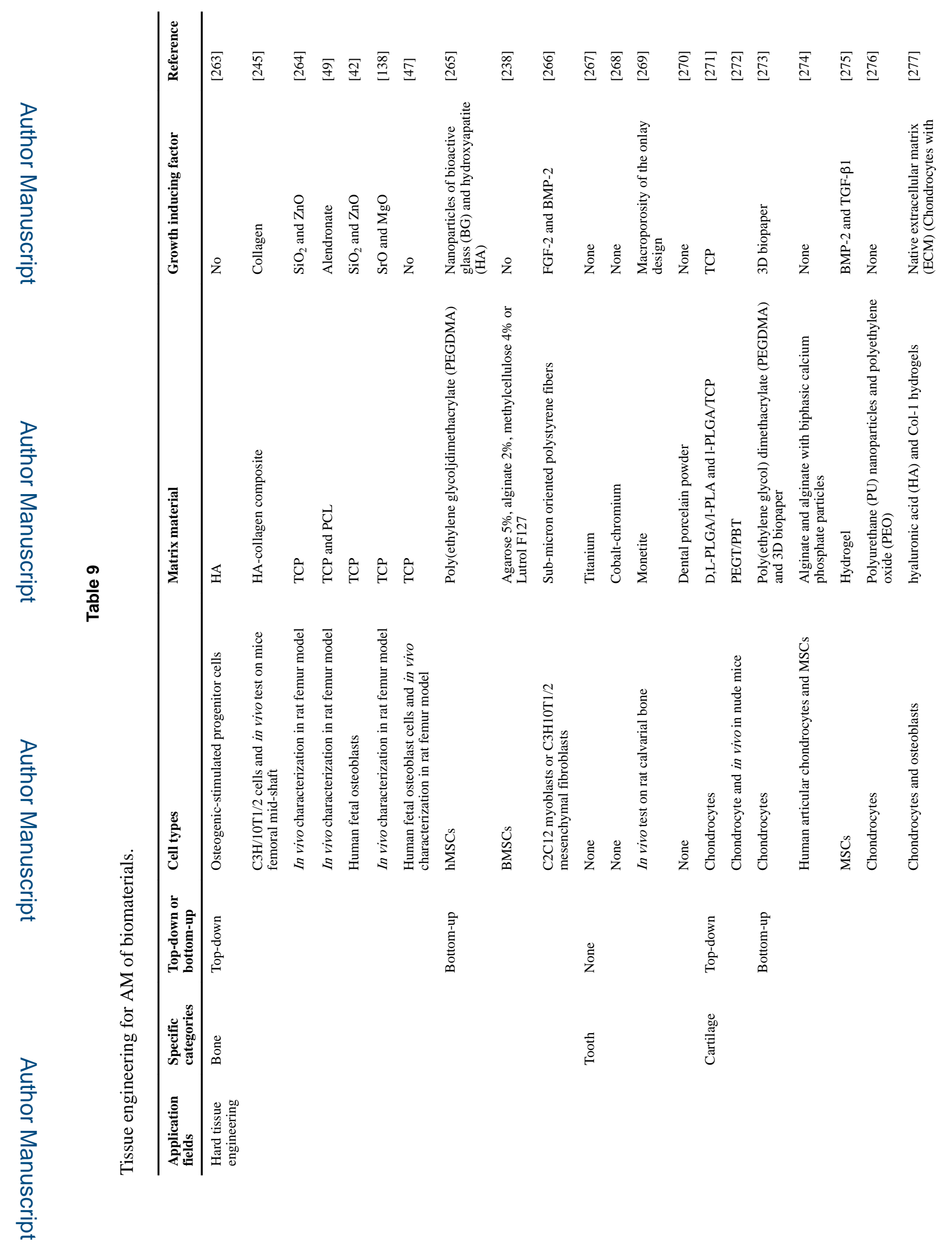

Prog Mater Sci. Author manuscript; available in PMC 2019 August 12. 
Bose et al.

Page 128

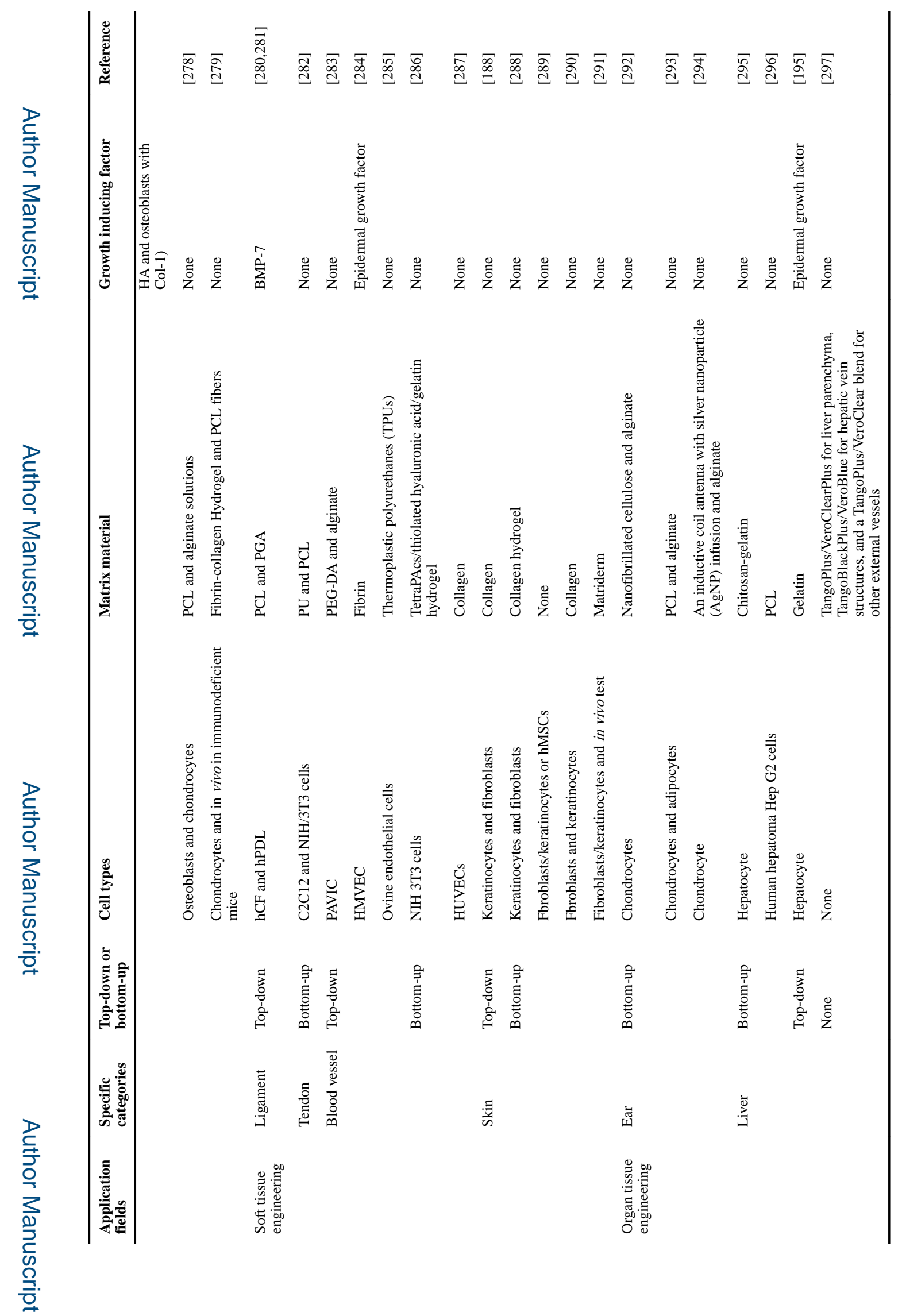

Prog Mater Sci. Author manuscript; available in PMC 2019 August 12. 